quatrième série-tome $46 \quad$ fascicule $4 \quad$ juillet-aồt 2013

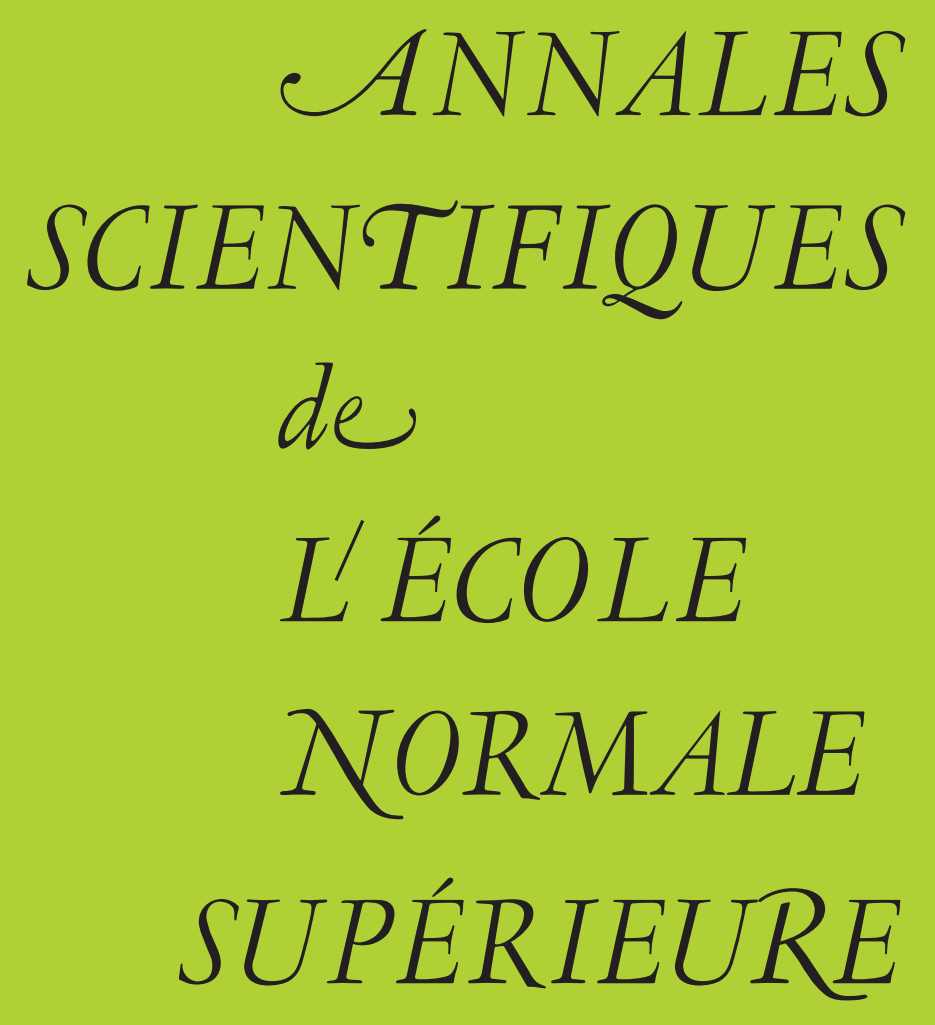

Carlos D'ANDREA \& Teresa KRICK \& Martín SOMBRA Heights of varieties in multiprojective spaces and aritbmetic Nullstellensätze 


\title{
HEIGHTS OF VARIETIES IN MULTIPROJECTIVE SPACES AND ARITHMETIC NULLSTELLENSÄTZE
}

\author{
By Carlos D'ANDREA, Teresa KRICK and Martín SOMBRA
}

Abstract. - We present bounds for the degree and the height of the polynomials arising in some problems in effective algebraic geometry including the implicitization of rational maps and the effective Nullstellensatz over a variety. Our treatment is based on arithmetic intersection theory in products of projective spaces and extends to the arithmetic setting constructions and results due to Jelonek. A key role is played by the notion of canonical mixed height of a multiprojective variety. We study this notion from the point of view of resultant theory and establish some of its basic properties, including its behavior with respect to intersections, projections and products. We obtain analogous results for the function field case, including a parametric Nullstellensatz.

RÉsumÉ. - Nous présentons des bornes pour les degrés et hauteurs des polynômes apparaissant dans certains problèmes de géométrie algébrique effective, dont l'implicitation d'applications rationnelles et le Nullstellensatz effectif sur une variété. Notre traitement est basé sur la théorie de l'intersection arithmétique dans un produit d'espaces projectifs. Il étend au cadre arithmétique des constructions et résultats dus à Jelonek. Un rôle central est joué par la notion de hauteur canonique mixte d'une variété multiprojective. Nous étudions cette notion à l'aide de la théorie des résultants et nous montrons quelques-unes de ses propriétés de base, y compris son comportement par rapport aux intersections, projections et produits. Nous obtenons aussi des résultats analogues dans le cas d'un corps de fonctions, dont un Nullstellensatz paramétrique.

D'Andrea was partially supported by the research project MTM2010-20279 (Spain). Krick was partially supported by the research projects ANPCyT 3671/05, UBACyT X-113 2008-2010 and CONICET PIP 2010-2012 (Argentina). Sombra was partially supported by the research projects MTM2009-14163-C02-01 and MTM201238122-C03-02 (Spain) and by a MinCyT Milstein fellowship (Argentina). 


\section{Introduction}

In 1983, Serge Lang wrote in the preface to his book [20]:

It is legitimate, and to many people an interesting point of view, to ask that the theorems of algebraic geometry from the Hilbert Nullstellensatz to the more advanced results should carry with them estimates on the coefficients occurring in these theorems. Although some of the estimates are routine, serious and interesting problems arise in this context.

Indeed, the main purpose of the present text is to give bounds for the degree and the size of the coefficients of the polynomials in the Nullstellensatz.

Let $f_{1}, \ldots, f_{s} \in \mathbb{Z}\left[x_{1}, \ldots, x_{n}\right]$ be polynomials without common zeros in the affine space $\mathbb{A}^{n}(\overline{\mathbb{Q}})$. The Nullstellensatz says then that there exist $\alpha \in \mathbb{Z} \backslash\{0\}$ and $g_{1}, \ldots, g_{s} \in \mathbb{Z}\left[x_{1}, \ldots, x_{n}\right]$ satisfying a Bézout identity

$$
\alpha=g_{1} f_{1}+\cdots+g_{s} f_{s} .
$$

As for many central results in commutative algebra and in algebraic geometry, it is a noneffective statement. By the end of the 1980s, the estimation of the degree and the height of polynomials satisfying such an identity became a widely considered question in connection with problems in computer algebra and Diophantine approximation. The results in this direction are generically known as arithmetic Nullstellensätze and they play an important role in number theory and in theoretical computer science. In particular, they apply to problems in complexity and computability $[16,1,9]$, to counting problems over finite fields or over the rationals [4, 34], and to effectivity in existence results in arithmetic geometry [17, 3].

The first non-trivial result on this problem was obtained by Philippon, who got a bound on the minimal size of the denominator $\alpha$ in a Bézout identity as above [28]. Berenstein and Yger achieved the next big progress, producing height estimates for the polynomials $g_{i}$ 's with techniques from complex analysis (integral formulae for residues of currents) [2]. Later on, Krick, Pardo and Sombra [19] exhibited sharp bounds by combining arithmetic intersection theory with the algebraic approach in [18] based on duality theory for Gorenstein algebras. Recall that the height of a polynomial $f \in \mathbb{Z}\left[x_{1}, \ldots, x_{n}\right]$, denoted by $\mathrm{h}(f)$, is defined as the logarithm of the maximum of the absolute value of its coefficients. Then, Theorem 1 in [19] reads as follows: if $d=\max _{j} \operatorname{deg}\left(f_{j}\right)$ and $h=\max _{j} \mathrm{~h}\left(f_{j}\right)$, there is a Bézout identity as above satisfying

$$
\operatorname{deg}\left(g_{i}\right) \leq 4 n d^{n}, \quad \mathrm{~h}(\alpha), \mathrm{h}\left(g_{i}\right) \leq 4 n(n+1) d^{n}(h+\log s+(n+7) \log (n+1) d) .
$$

We refer the reader to the surveys [38, 6] for further information on the history of the effective Nullstellensatz, main results and open questions.

One of the main results of this text is the arithmetic Nullstellensatz over a variety below, which is a particular case of Theorem 4.28. For an affine equidimensional variety $V \subset \mathbb{A}^{n}(\overline{\mathbb{Q}})$, we denote by $\operatorname{deg}(V)$ and by $\widehat{\mathrm{h}}(V)$ the degree and the canonical height of the closure of $V$ with respect to the standard inclusion $\mathbb{A}^{n} \hookrightarrow \mathbb{P}^{n}$. The degree and the height of a variety are measures of its geometric and arithmetic complexity, see $\$ 2.3$ and the references therein for details. We say that a polynomial relation holds on a variety if it holds for every point in it.

4 e SÉRIE - TOME $46-2013-$ No $^{\circ}$ 
Theorem 1. - Let $V \subset \mathbb{A}^{n}(\overline{\mathbb{Q}})$ be a variety defined over $\mathbb{Q}$ of pure dimension $r$ and $f_{1}, \ldots, f_{s} \in \mathbb{Z}\left[x_{1}, \ldots, x_{n}\right] \backslash \mathbb{Z}$ a family of $s \leq r+1$ polynomials without common zeros in $V$. Set $d_{j}=\operatorname{deg}\left(f_{j}\right)$ and $h_{j}=\mathrm{h}\left(f_{j}\right)$ for $1 \leq j \leq s$. Then there exist $\alpha \in \mathbb{Z} \backslash\{0\}$ and $g_{1}, \ldots, g_{s} \in \mathbb{Z}\left[x_{1}, \ldots, x_{n}\right]$ such that

$$
\alpha=g_{1} f_{1}+\cdots+g_{s} f_{s} \quad \text { on } V
$$

with

$$
\begin{aligned}
& \text { - } \operatorname{deg}\left(g_{i} f_{i}\right) \leq\left(\prod_{j=1}^{s} d_{j}\right) \operatorname{deg}(V), \\
& \text { - } \mathrm{h}(\alpha), \mathrm{h}\left(g_{i}\right)+\mathrm{h}\left(f_{i}\right) \leq\left(\prod_{j=1}^{s} d_{j}\right)\left(\widehat{\mathrm{h}}(V)+\operatorname{deg}(V)\left(\sum_{\ell=1}^{s} \frac{h_{\ell}}{d_{\ell}}+(4 r+8) \log (n+3)\right)\right) .
\end{aligned}
$$

For $V=\mathbb{A}^{n}$, this result gives the bounds

$$
\operatorname{deg}\left(g_{i} f_{i}\right) \leq \prod_{j=1}^{s} d_{j}, \quad \mathrm{~h}(\alpha), \mathrm{h}\left(g_{i}\right)+\mathrm{h}\left(f_{i}\right) \leq \sum_{\ell=1}^{s}\left(\prod_{j \neq \ell} d_{j}\right) h_{\ell}+(4 n+8) \log (n+3) \prod_{j=1}^{s} d_{j} .
$$

These bounds are substantially sharper than the previously known. Moreover, they are close to optimal in many situations. For instance, let $d_{1}, \ldots, d_{n+1}, H \geq 1$ and set

$$
f_{1}=x_{1}-H, f_{2}=x_{2}-x_{1}^{d_{2}}, \ldots, f_{n}=x_{n}-x_{n-1}^{d_{n}}, f_{n+1}=x_{n}^{d_{n+1}} .
$$

This is a system of polynomials without common zeros. Hence, the above result implies that there is a Bézout identity $\alpha=g_{1} f_{1}+\cdots+g_{n+1} f_{n+1}$ which satisfies $\mathrm{h}(\alpha) \leq d_{2} \cdots d_{n+1}(\log (H)+(4 n+8) \log (n+3))$. On the other hand, specializing any such identity at the point $\left(H, H^{d_{2}}, \ldots, H^{d_{2} \cdots d_{n}}\right)$, we get

$$
\alpha=g_{n+1}\left(H, H^{d_{2}}, \ldots, H^{d_{2} \cdots d_{n}}\right) H^{d_{2} \cdots d_{n+1}} .
$$

This implies the lower bound $\mathrm{h}(\alpha) \geq d_{2} \cdots d_{n+1} \log (H)$ and shows that the height bound in Theorem 1 is sharp in this case. More examples can be found in $\S 4.3$.

It is important to mention that all previous results in the literature are limited to the case when $V$ is a complete intersection and cannot properly distinguish the influence of each individual $f_{j}$, due to the limitations of the methods applied. Hence, Theorem 1 is a big progress as it holds for an arbitrary variety and gives bounds depending on the degree and height of each $f_{j}$. This last point is more important than it might seem at first. Indeed, by using Rabinowicz' trick one can show that the weak Nullstellensatz implies its strong version. However, this reduction yields good bounds for the strong Nullstellensatz only if the corresponding weak version can correctly differentiate the influence of each $f_{j}$, see Remark 4.27. Using this observation, we obtain in $\$ 4.3$ the following arithmetic version of the strong Nullstellensatz over a variety.

Theorem 2. - Let $V \subset \mathbb{A}^{n}(\overline{\mathbb{Q}})$ be a variety defined over $\mathbb{Q}$ of pure dimension $r$ and $g, f_{1}, \ldots, f_{s} \in \mathbb{Z}\left[x_{1}, \ldots, x_{n}\right]$ such that $g$ vanishes on the common zeros of $f_{1}, \ldots, f_{s}$ in $V$. Set $d_{j}=\operatorname{deg}\left(f_{j}\right)$ and $h=\max _{j} \mathrm{~h}\left(f_{j}\right)$ for $1 \leq j \leq s$. Assume that $d_{1} \geq \cdots \geq d_{s} \geq 1$ and set $D=\prod_{j=1}^{\min \{s, r+1\}} d_{j}$. Set also $d_{0}=\max \{1, \operatorname{deg}(g)\}$ and $h_{0}=\mathrm{h}(g)$. Then there exist $\mu \in \mathbb{N}$, $\alpha \in \mathbb{Z} \backslash\{0\}$ and $g_{1}, \ldots, g_{s} \in \mathbb{Z}\left[x_{1}, \ldots, x_{n}\right]$ such that

$$
\alpha g^{\mu}=g_{1} f_{1}+\cdots+g_{s} f_{s} \quad \text { on } V
$$


with

- $\mu \leq 2 D \operatorname{deg}(V)$

- $\operatorname{deg}\left(g_{i} f_{i}\right) \leq 4 d_{0} D \operatorname{deg}(V)$,

- $\mathrm{h}(\alpha), \mathrm{h}\left(g_{i}\right)+\mathrm{h}\left(f_{i}\right) \leq 2 d_{0} D\left(\widehat{\mathrm{h}}(V)+\operatorname{deg}(V)\left(\frac{3 h_{0}}{2 d_{0}}+\sum_{\ell=1}^{\min \{s, r+1\}} \frac{h}{d_{\ell}}+c(n, r, s)\right)\right)$,

where $c(n, r, s) \leq(6 r+17) \log (n+4)+3(r+1) \log (\max \{1, s-r\})$.

Our treatment of this problem is the arithmetic counterpart of Jelonek's approach to produce bounds for the degrees in the Nullstellensatz over a variety [14]. To this end, we develop a number of tools in arithmetic intersection and elimination theory in products of projective spaces. A key role is played by the notion of canonical mixed heights of multiprojective varieties, which we study from the point of view of resultants. Our presentation of mixed resultants of cycles in multiprojective spaces is mostly a reformulation of the theory developed by Rémond in $[32,33]$ as an extension of Philippon's theory of eliminants of homogeneous ideals [27]. We also establish new properties of them, including their behavior under projections (Proposition 1.41) and products (Proposition 1.45).

Let $\boldsymbol{n}=\left(n_{1}, \ldots, n_{m}\right) \in \mathbb{N}^{m}$ and set $\mathbb{P}^{\boldsymbol{n}}=\mathbb{P}^{n_{1}}(\overline{\mathbb{Q}}) \times \cdots \times \mathbb{P}^{n_{m}}(\overline{\mathbb{Q}})$ for the corresponding multiprojective space. For a cycle $X$ of $\mathbb{P}^{n}$ of pure dimension $r$ and a multi-index $\boldsymbol{c}=\left(c_{1}, \ldots, c_{m}\right) \in \mathbb{N}^{m}$ of length $r+1$, the mixed Fubini-Study height $\mathrm{h}_{\boldsymbol{c}}(X)$ is defined as an alternative Mahler measure of the corresponding mixed resultant (Definition 2.40). The canonical mixed height is then defined by a limit process as

$$
\widehat{\mathrm{h}}_{c}(X):=\lim _{\ell \rightarrow \infty} \ell^{-r-1} \mathrm{~h}_{c}\left([\ell]_{*} X\right),
$$

where $[\ell]$ denotes the $\ell$-power map of $\mathbb{P}^{n}$ (Proposition-Definition 2.45).

To handle mixed degrees and heights, we introduce a notion of extended Chow ring of $\mathbb{P}^{n}$ (Definition 2.50). It is an arithmetic analogue of the Chow ring of $\mathbb{P}^{\boldsymbol{n}}$ and can be identified with the quotient ring $\mathbb{R}\left[\eta, \theta_{1}, \ldots, \theta_{m}\right] /\left(\eta^{2}, \theta_{1}^{n_{1}+1}, \ldots, \theta_{m}^{n_{m}+1}\right)$. We associate to the cycle $X$ an element in this ring, denoted by $[X]_{\mathbb{Z}}$, corresponding under this identification to

$$
\sum_{\boldsymbol{c}} \widehat{\mathrm{h}}_{\boldsymbol{c}}(X) \eta \theta_{1}^{n_{1}-c_{1}} \cdots \theta_{m}^{n_{m}-c_{m}}+\sum_{\boldsymbol{b}} \operatorname{deg}_{\boldsymbol{b}}(X) \theta_{1}^{n_{1}-b_{1}} \cdots \theta_{m}^{n_{m}-b_{m}}
$$

the sums being indexed by all $\boldsymbol{b}, \boldsymbol{c} \in \mathbb{N}^{m}$ of respective lengths $r$ and $r+1$ such that $\boldsymbol{b}, \boldsymbol{c} \leq \boldsymbol{n}$. Here, $\operatorname{deg}_{\boldsymbol{b}}(X)$ denotes the mixed degree of $X$ of index $\boldsymbol{b}$. This element contains the information of all non-trivial mixed degrees and canonical mixed heights of $X$, since $\operatorname{deg}_{\boldsymbol{b}}(X)$ and $\widehat{\mathrm{h}}_{\boldsymbol{c}}(X)$ are zero for any other $\boldsymbol{b}$ and $\boldsymbol{c}$.

The extended Chow ring of $\mathbb{P}^{n}$ turns out to be a quite useful object which allows to translate geometric operations on multiprojective cycles into algebraic operations on rings and classes. In particular, we obtain the following multiprojective arithmetic Bézout's inequality, see also Theorem 2.58. For a multihomogeneous polynomial $f \in \mathbb{Z}\left[\boldsymbol{x}_{1}, \ldots, \boldsymbol{x}_{m}\right]$, where $\boldsymbol{x}_{i}$ is a group of $n_{i}+1$ variables, we denote by $\|f\|_{\text {sup }}$ its sup-norm (Definition 2.29) and consider the element $[f]_{\text {sup }}$ in the extended Chow ring corresponding to the element $\sum_{i=1}^{m} \operatorname{deg}_{\boldsymbol{x}_{i}}(f) \theta_{i}+\log \|f\|_{\text {sup }} \eta$. 
Theorem 3. - Let $X$ be an effective equidimensional cycle of $\mathbb{P}^{\boldsymbol{n}}$ defined over $\mathbb{Q}$ and $f \in$ $\mathbb{Z}\left[\boldsymbol{x}_{1}, \ldots, \boldsymbol{x}_{m}\right]$ a multihomogeneous polynomial such that $X$ and $\operatorname{div}(f)$ intersect properly. Then

$$
[X \cdot \operatorname{div}(f)]_{\mathbb{Z}} \leq[X]_{\mathbb{Z}} \cdot[f]_{\mathrm{sup}} .
$$

Statements on classes in the extended Chow ring can easily be translated into statements on mixed degrees and heights. In this direction, the above result implies that, for any $\boldsymbol{b} \in \mathbb{N}^{m}$ of length equal to $\operatorname{dim}(X)$,

$$
\widehat{\mathrm{h}}_{\boldsymbol{b}}(X \cdot \operatorname{div}(f)) \leq \sum_{i=1}^{m} \operatorname{deg}_{\boldsymbol{x}_{i}}(f) \widehat{\mathrm{h}}_{\boldsymbol{b}+\boldsymbol{e}_{i}}(X)+\log \|f\|_{\text {sup }} \operatorname{deg}_{\boldsymbol{b}}(X)
$$

where $\boldsymbol{e}_{i}$ denotes the $i$-th vector of the standard basis of $\mathbb{R}^{m}$. In a similar way, we also study the behavior of arithmetic classes (and a fortiori, of canonical mixed heights) under projections (Proposition 2.64) and products (Proposition 2.66), among other results.

Jelonek's approach consists in producing a Bézout identity from an implicit equation of a specific regular map. In general, the implicitization problem consists in computing equations for an algebraic variety $W$ from a given rational parameterization of it. The typical case is when $W$ is a hypersurface: the variety is then defined by a single equation and the problem consists in computing this "implicit equation". We consider here the problem of estimating the height of the implicit equation of a hypersurface parameterized by a regular map $V \rightarrow W$ whose domain is an affine variety $V$, in terms of the degree and the height of $V$ and of the polynomials defining the map. To this end, we prove the following arithmetic version of Perron's theorem over a variety [14, Thm. 3.3]. It is obtained as a consequence of Theorem 3.15.

Theorem 4. - Let $V \subset \mathbb{A}^{n}(\overline{\mathbb{Q}})$ be a variety defined over $\mathbb{Q}$ of pure dimension $r$. Let $q_{1}, \ldots, q_{r+1} \in \mathbb{Z}\left[x_{1}, \ldots, x_{n}\right] \backslash \mathbb{Z}$ such that the closure of the image of the map

$$
V \longrightarrow \mathbb{A}^{r+1}(\overline{\mathbb{Q}}), \quad \boldsymbol{x} \longmapsto\left(q_{1}(\boldsymbol{x}), \ldots, q_{r+1}(\boldsymbol{x})\right)
$$

is a hypersurface. Let $E=\sum_{\boldsymbol{a} \in \mathbb{N}^{r+1}} \alpha_{\boldsymbol{a}} \boldsymbol{y}^{\boldsymbol{a}} \in \mathbb{Z}\left[y_{1}, \ldots, y_{r+1}\right]$ be a primitive and squarefree polynomial defining this hypersurface. Set $d_{j}=\operatorname{deg}\left(q_{j}\right), h_{j}=\mathrm{h}\left(q_{j}\right)$ for $1 \leq j \leq r+1$. Then, for all $\boldsymbol{a}=\left(a_{1}, \ldots, a_{r+1}\right)$ such that $\alpha_{\boldsymbol{a}} \neq 0$,

$$
\begin{aligned}
& \bullet \sum_{i=1}^{r+1} a_{i} d_{i} \leq\left(\prod_{j=1}^{r+1} d_{j}\right) \operatorname{deg}(V), \\
& \bullet \mathrm{h}\left(\alpha_{\boldsymbol{a}}\right)+\sum_{i=1}^{r+1} a_{i} h_{i} \leq\left(\prod_{j=1}^{r+1} d_{j}\right)\left(\widehat{\mathrm{h}}(V)+\operatorname{deg}(V)\left(\sum_{\ell=1}^{r+1} \frac{h_{\ell}}{d_{\ell}}+(r+2) \log (n+3)\right)\right) .
\end{aligned}
$$

For $V=\mathbb{A}^{n}$ we have $r=n, \operatorname{deg}(V)=1$ and $\widehat{\mathrm{h}}(V)=0$. Hence, the above result extends the classical Perron's theorem [26, Satz 57], which amounts to the weighted degree bound for the implicit equation $\sum_{i} a_{i} d_{i} \leq \prod_{j} d_{j}$, by adding the bound for the height

$$
\mathrm{h}\left(\alpha_{\boldsymbol{a}}\right)+\sum_{i=1}^{n+1} a_{i} h_{i} \leq \sum_{\ell=1}^{n+1}\left(\prod_{j \neq \ell} d_{j}\right) h_{\ell}+(n+2) \log (n+3) \prod_{j=1}^{n+1} d_{j} .
$$


Our results on the implicitization problem as well as those on mixed resultants and multiprojective arithmetic intersection theory should be of independent interest, besides their applications to the arithmetic Nullstellensatz.

The method is not exclusive of $\mathbb{Z}$ but can also be carried over to other rings equipped with a suitable height function. In this direction, we apply it to $k\left[t_{1}, \ldots, t_{p}\right]$, the ring of polynomials over an arbitrary field $k$ in $p$ variables: if we set $t=\left\{t_{1}, \ldots, t_{p}\right\}$, the height of a polynomial with coefficients in $k[\boldsymbol{t}]$ is its degree in the variables $\boldsymbol{t}$. For this case, we also develop the corresponding arithmetic intersection theory, including the behavior of classes in the extended Chow ring with respect to intersections (Theorem 2.18), projections (Proposition 2.22), products (Proposition 2.23) and ruled joins (Proposition 2.24). As a consequence, we obtain a parametric analogue of Perron's theorem (Theorem 3.1) and then the parametric Nullstellensatz below, which is a particular case of Theorem 4.11. For an affine equidimensional variety $V \subset \mathbb{A}^{n}(\overline{k(t)})$, we denote by $\mathrm{h}(V)$ the $\boldsymbol{t}$-degree of the Chow form of its closure in $\mathbb{P}^{n}(\overline{k(t)})$, see $\$ 2.1$ for details.

TheOREm 5. - Let $V \subset \mathbb{A}^{n}(\overline{k(\boldsymbol{t})})$ be a variety defined over $k(\boldsymbol{t})$ of pure dimension $r$ and $f_{1}, \ldots, f_{s} \in k[\boldsymbol{t}]\left[x_{1}, \ldots, x_{n}\right] \backslash k[\boldsymbol{t}]$ a family of $s \leq r+1$ polynomials without common zeros in $V$. Set $d_{j}=\operatorname{deg}_{\boldsymbol{x}}\left(f_{j}\right)$ and $h_{j}=\operatorname{deg}_{\boldsymbol{t}}\left(f_{j}\right)$ for $1 \leq j \leq s$. Then there exist $\alpha \in k[\boldsymbol{t}] \backslash\{0\}$ and $g_{1}, \ldots, g_{s} \in k[\boldsymbol{t}]\left[x_{1}, \ldots, x_{n}\right]$ such that

$$
\alpha=g_{1} f_{1}+\cdots+g_{s} f_{s} \quad \text { on } V
$$

with

$$
\begin{aligned}
& \text { - } \operatorname{deg}_{\boldsymbol{x}}\left(g_{i} f_{i}\right) \leq\left(\prod_{j=1}^{s} d_{j}\right) \operatorname{deg}(V), \\
& \text { - } \operatorname{deg}(\alpha), \operatorname{deg}_{\boldsymbol{t}}\left(g_{i} f_{i}\right) \leq\left(\prod_{j=1}^{s} d_{j}\right)\left(\mathrm{h}(V)+\operatorname{deg}(V) \sum_{\ell=1}^{s} \frac{h_{\ell}}{d_{\ell}}\right) .
\end{aligned}
$$

For $V=\mathbb{A}^{n}(\overline{k(t)})$ we have $r=n, \operatorname{deg}(V)=1$ and $\mathrm{h}(V)=0$. Hence, this result gives the following bounds for the partial degrees of the polynomials in a Bézout identity:

$$
\operatorname{deg}_{\boldsymbol{x}}\left(g_{i} f_{i}\right) \leq \prod_{j=1}^{s} d_{j}, \quad \operatorname{deg}(\alpha), \operatorname{deg}_{\boldsymbol{t}}\left(g_{i} f_{i}\right) \leq \sum_{\ell=1}^{s}\left(\prod_{j \neq \ell} d_{j}\right) h_{\ell} .
$$

In Theorem 4.22, we give a strong version of the parametric Nullstellensatz over a variety, which also contains the case of an arbitrary number of input polynomials. Up to our knowledge, the only previous results on the parametric Nullstellensatz are due to Smietanski [35], who considers the case when the number of parameters $p$ is at most two and $V=\mathbb{A}^{n}(\overline{k(t)})$, see Remark 4.21.

To prove both the arithmetic and parametric versions of the effective Nullstellensatz, we need to consider a more general version of these statements where the input polynomials depend on groups of parameters, see Theorem 4.28. The latter has further interesting applications. For instance, consider the family $F_{1}, \ldots, F_{n+1}$ of general $n$-variate polynomials of degree $d_{1}, \ldots, d_{n+1}$, respectively. For each $j$, write

$$
F_{j}=\sum_{a} u_{j, a} x^{a}
$$

$4^{\mathrm{e}}$ SÉRIE - TOME $46-2013-\mathrm{N}^{\circ} 4$ 
where each $u_{j, \boldsymbol{a}}$ is a variable. Let $\boldsymbol{u}_{j}=\left\{u_{j, \boldsymbol{a}}\right\}_{\boldsymbol{a}}$ be the group of variables corresponding to the coefficients of $F_{j}$ and set $\boldsymbol{u}=\left\{\boldsymbol{u}_{1}, \ldots, \boldsymbol{u}_{n+1}\right\}$. The corresponding Macaulay resultant $R \in \mathbb{Z}[\boldsymbol{u}]$ lies in the ideal $\left(F_{1}, \ldots, F_{n+1}\right) \subset \mathbb{Q}[\boldsymbol{u}, \boldsymbol{x}]$ and Theorem 4.28 gives bounds for a representation of $R$ in this ideal. Indeed, we obtain that there are $\lambda \in \mathbb{Z} \backslash\{0\}$ and $g_{j} \in \mathbb{Z}[\boldsymbol{u}, \boldsymbol{x}]$ such that $\lambda R=g_{1} F_{1}+\cdots+g_{n+1} F_{n+1}$ with

$$
\operatorname{deg}_{\boldsymbol{u}_{j}}\left(g_{i} F_{i}\right) \leq \prod_{\ell \neq j} d_{\ell}, \quad \mathrm{h}(\lambda R), \mathrm{h}\left(g_{i}\right) \leq(6 n+10) \log (n+3)\left(\prod_{\ell=1}^{n+1} d_{\ell}\right),
$$

see Examples 4.18 and 4.37. The obtained bound for the height of the $g_{i}$ 's is of the same order as the sharpest known bounds for the height of $R$, see [37].

This text is divided in four sections. In the first one, we recall the basic properties of mixed resultants and degrees of cycles in multiprojective spaces over an arbitrary field $K$. The second section focuses on the mixed heights of cycles for the case when $K$ is a function field and on the canonical mixed heights of cycles for $K=\mathbb{Q}$. In the third section, we apply this machinery to the study of the height of the implicit equation, including generalizations and variants of Theorem 4 . We conclude in the fourth section by deriving the different arithmetic Nullstellensätze.

Acknowledgments. - We thank José Ignacio Burgos for the many discussions we had and, in particular, for the statement and the proof of Lemma 1.18. We thank Teresa Cortadellas, Santiago Laplagne and Juan Carlos Naranjo for helpful discussions and Matilde Lalín for pointing us some references on Mahler measures. We also thank the referee for his/her comments and, especially, for a simplification of our argument in the proof of Lemma 1.29. D'Andrea thanks the University of Bordeaux 1 for inviting him in February 2009, Krick thanks the University of Buenos Aires for her sabbatical during 2009 and the Universities of Bordeaux 1, Caen, Barcelona and Nice-Sophia Antipolis for hosting her during that time, and Sombra thanks the University of Buenos Aires for inviting him during OctoberDecember 2007 and November 2010. The three authors also thank the Fields Institute, where they met during the Fall 2009 FoCM thematic program.

\section{Degrees and resultants of multiprojective cycles}

Throughout this text, we denote by $\mathbb{N}=\mathbb{Z}_{\geq 0}$ and by $\mathbb{Z}_{>0}$ the sets of non-negative and positive integers, respectively. Bold letters denote finite sets or sequences of objects, where the type and number should be clear from the context: for instance, $\boldsymbol{x}$ might denote $\left\{x_{1}, \ldots, x_{n}\right\}$ so that if $A$ is a ring, $A[\boldsymbol{x}]=A\left[x_{1}, \ldots, x_{n}\right]$. For a polynomial $f \in A[\boldsymbol{x}]$ we adopt the usual notation

$$
f=\sum_{a} \alpha_{a} x^{a}
$$

where, for each index $\boldsymbol{a}=\left(a_{1}, \ldots, a_{n}\right) \in \mathbb{N}^{n}, \alpha_{\boldsymbol{a}}$ denotes an element of $A$ and $\boldsymbol{x}^{\boldsymbol{a}}$ the monomial $x_{1}^{a_{1}} \cdots x_{n}^{a_{n}}$. For $\boldsymbol{a} \in \mathbb{N}^{n}$, we denote by $|\boldsymbol{a}|=a_{1}+\cdots+a_{n}$ its length and by coeff $\boldsymbol{a}(f)=\alpha_{\boldsymbol{a}}$ the coefficient of $\boldsymbol{x}^{\boldsymbol{a}}$. We also set $\boldsymbol{a} !=a_{1} ! \cdots a_{n}$ !. The support of $f$ is the set of exponents corresponding to its non-zero terms, that is, $\operatorname{supp}(f)=\left\{\boldsymbol{a}:\right.$ coeff $\left._{\boldsymbol{a}}(f) \neq 0\right\} \subset \mathbb{N}^{n}$. For $\boldsymbol{a}, \boldsymbol{b} \in \mathbb{R}^{n}$, we set $\langle\boldsymbol{a}, \boldsymbol{b}\rangle=\sum_{i=1}^{n} a_{i} b_{i}$. We say that $\boldsymbol{a} \leq \boldsymbol{b}$ whenever the inequality holds coefficient-wise. 
For a factorial ring $A$, we denote by $A^{\times}$its group of units. A polynomial with coefficients in $A$ is primitive if its coefficients have no common factor in $A \backslash A^{\times}$.

\subsection{Preliminaries on multiprojective geometry}

Let $A$ be a factorial ring with field of fractions $K$ and $\bar{K}$ the algebraic closure of $K$. For $m \in \mathbb{Z}_{>0}$ and $\boldsymbol{n}=\left(n_{1}, \ldots, n_{m}\right) \in \mathbb{N}^{m}$ we consider the multiprojective space over $\bar{K}$

$$
\mathbb{P}^{\boldsymbol{n}}(\bar{K})=\mathbb{P}^{n_{1}}(\bar{K}) \times \cdots \times \mathbb{P}^{n_{m}}(\bar{K}) .
$$

We also write $\mathbb{P}^{\boldsymbol{n}}=\mathbb{P}^{\boldsymbol{n}}(\bar{K})$ for short. For $1 \leq i \leq m$, let $\boldsymbol{x}_{i}=\left\{x_{i, 0}, \ldots, x_{i, n_{i}}\right\}$ be a group of $n_{i}+1$ variables and set

$$
\boldsymbol{x}=\left\{\boldsymbol{x}_{1}, \ldots, \boldsymbol{x}_{m}\right\}
$$

The multihomogeneous coordinate ring of $\mathbb{P}^{\boldsymbol{n}}$ is $\bar{K}[\boldsymbol{x}]=\bar{K}\left[\boldsymbol{x}_{1}, \ldots, \boldsymbol{x}_{m}\right]$. It is multigraded by declaring $\operatorname{deg}\left(x_{i, j}\right)=\boldsymbol{e}_{i} \in \mathbb{N}^{m}$, the $i$-th vector of the standard basis of $\mathbb{R}^{m}$. For $\boldsymbol{d}=\left(d_{1}, \ldots, d_{m}\right) \in \mathbb{N}^{m}$, we denote by $\bar{K}[\boldsymbol{x}]_{\boldsymbol{d}}$ its part of multidegree $\boldsymbol{d}$. Set

$$
\mathbb{N}_{d_{i}}^{n_{i}+1}=\left\{\boldsymbol{a}_{i} \in \mathbb{N}^{n_{i}+1}:\left|\boldsymbol{a}_{i}\right|=d_{i}\right\}, \quad \mathbb{N}_{\boldsymbol{d}}^{\boldsymbol{n}+\mathbf{1}}=\prod_{1 \leq i \leq m} \mathbb{N}_{d_{i}}^{n_{i}+1}
$$

A multihomogeneous polynomial $f \in \bar{K}[\boldsymbol{x}]_{\boldsymbol{d}}$ can then be written down as

$$
f=\sum_{\boldsymbol{a} \in \mathbb{N}_{d}^{n+1}} \alpha_{\boldsymbol{a}} \boldsymbol{x}^{\boldsymbol{a}} .
$$

Let $K \subset E$ be an extension of fields and $f \in E[\boldsymbol{x}]_{\boldsymbol{d}}$. For a point $\boldsymbol{\xi} \in \mathbb{P}^{\boldsymbol{n}}$, the value $f(\boldsymbol{\xi})$ is only defined up to a non-zero scalar in $\bar{K}^{\times}$which depends on a choice of multihomogeneous coordinates for $\boldsymbol{\xi}$.

An ideal $I \subset \bar{K}[\boldsymbol{x}]$ is multihomogeneous if it is generated by a family of multihomogeneous polynomials. For any such ideal, we denote by $V(I)$ the subvariety of $\mathbb{P}^{\boldsymbol{n}}$ defined as its set of zeros. Along this text, a variety is neither necessarily irreducible nor equidimensional. Reciprocally, given a variety $V \subset \mathbb{P}^{n}$, we denote by $I(V)$ the multihomogeneous ideal of $\bar{K}[\boldsymbol{x}]$ of polynomials vanishing on $V$. A variety $V$ is defined over $K$ if its defining ideal $I(V)$ is generated by polynomials in $K[\boldsymbol{x}]$.

Let $\mathfrak{M}_{\boldsymbol{n}}=\left\{x_{1, j_{1}} \cdots x_{m, j_{m}}: 0 \leq j_{i} \leq n_{i}\right\}$ be the set of monomials of multidegree $(1, \ldots, 1) \in \mathbb{N}^{m}$. A multihomogeneous ideal $I \subset \bar{K}[\boldsymbol{x}]$ defines the empty variety of $\mathbb{P}^{\boldsymbol{n}}$ if and only if $\mathfrak{M}_{\boldsymbol{n}} \subset \sqrt{I}$, see for instance [32, Lem. 2.9]. The assignment $V \mapsto I(V)$ is a one-toone correspondence between non-empty subvarieties of $\mathbb{P}^{n}$ and radical multihomogeneous ideals of $\bar{K}[\boldsymbol{x}]$ not containing $\mathfrak{M}_{\boldsymbol{n}}$.

More generally, we denote by $\mathbb{P}_{K}^{n}$ the multiprojective space over $K$ corresponding to $\boldsymbol{n}$. The reduced subschemes of $\mathbb{P}_{K}^{n}$ will be alternatively called subvarieties of $\mathbb{P}_{K}^{n}$ or $K$-varieties. There is a one-to-one correspondence $V \mapsto I(V)$ between non-empty subvarieties of $\mathbb{P}_{K}^{n}$ and radical multihomogeneous ideals of $K[\boldsymbol{x}]$ not containing $\mathfrak{M}_{\boldsymbol{n}}$. For a multihomogeneous ideal $I \subset K[\boldsymbol{x}]$ not containing $\mathfrak{M}_{\boldsymbol{n}}$, we denote by $V(I)$ its corresponding $K$-variety. A $K$-variety $V$ is irreducible if it is an integral subscheme of $\mathbb{P}_{K}^{n}$ or, equivalently, if the ideal $I(V)$ is prime. The dimension of $V$ coincides with the Krull dimension of the algebra $K\left[\boldsymbol{x}_{1}, \ldots, \boldsymbol{x}_{m}\right] / I(V)$ minus $m$.

$4^{\text {e }}$ SÉRIE - TOME $46-2013$ - No 4 
REMARK 1.1. - In the algebraically closed case, the scheme $\mathbb{P}_{K}^{n}$ can be identified with the set of points $\mathbb{P}^{\boldsymbol{n}}(\bar{K})$ and a subvariety $V \subset \mathbb{P}_{\bar{K}}^{\boldsymbol{n}}$ can be identified with its set of points $V(\bar{K}) \subset \mathbb{P}^{n}(\bar{K})$. Under this identification, a subvariety of $\mathbb{P}^{\boldsymbol{n}}(\bar{K})$ defined over $K$ corresponds to a $K$-variety. However, a $K$-variety does not necessarily correspond to a subvariety of $\mathbb{P}^{n}(\bar{K})$ defined over $K$, as the following example shows. Let $t$ be a variable and set $K=\mathbb{F}_{p}(t)$, where $p$ is a prime number and $\mathbb{F}_{p}$ is the field with $p$ elements. The ideal $\left(x_{1}^{p}-t x_{0}^{p}\right) \subset K\left[x_{0}, x_{1}\right]$ is prime and hence gives a subvariety of $\mathbb{P}_{K}^{1}$. Its set of zeros in $\mathbb{P}^{1}(\bar{K})$ consists in the point $\left\{\left(1: t^{1 / p}\right)\right\}$, which is not a variety defined over $K$. When the field $K$ is perfect (for instance, if $\operatorname{char}(K)=0$ ), the notion of $K$-variety does coincide, under this identification, with the notion of subvariety of $\mathbb{P}^{n}(\bar{K})$ defined over $K$.

A $K$-cycle of $\mathbb{P}_{K}^{n}$ is a finite $\mathbb{Z}$-linear combination

$$
X=\sum_{V} m_{V} V
$$

of irreducible subvarieties of $\mathbb{P}_{K}^{n}$. The subvarieties $V$ such that $m_{V} \neq 0$ are the irreducible components of $X$. A $K$-cycle is of pure dimension or equidimensional if its components are all of the same dimension. It is effective (respectively, reduced) if it can be written as in (1.2) with $m_{V} \geq 0$ (respectively, $m_{V}=1$ ). Given two $K$-cycles $X_{1}$ and $X_{2}$, we say that $X_{1} \geq X_{2}$ whenever $X_{1}-X_{2}$ is effective. The support of $X$, denoted $|X|$, is the $K$-variety defined as the union of its components. Reciprocally, a $K$-variety is a union of irreducible $K$-varieties of $\mathbb{P}^{n}$ and we identify it with the reduced $K$-cycle given as the sum of these irreducible $K$-varieties.

For $0 \leq r \leq|\boldsymbol{n}|$, we denote by $Z_{r}\left(\mathbb{P}_{K}^{\boldsymbol{n}}\right)$ the group of $K$-cycles of $\mathbb{P}^{\boldsymbol{n}}$ of pure dimension $r$ and by $Z_{r}^{+}\left(\mathbb{P}_{K}^{n}\right)$ the semigroup of those which are effective. For shorthand, a $\bar{K}$-cycle is called a cycle and we denote the sets of $\bar{K}$-cycles and of effective $\bar{K}$-cycles of pure dimension $r$ as $Z_{r}\left(\mathbb{P}^{\boldsymbol{n}}\right)$ and as $Z_{r}^{+}\left(\mathbb{P}^{\boldsymbol{n}}\right)$, respectively.

Let $I \subset K[\boldsymbol{x}]$ be a multihomogeneous ideal. For each minimal prime ideal $P$ of $I$, we denote by $m_{P}$ the multiplicity of $P$ in $I$, defined as the length of the $K[\boldsymbol{x}]_{P}$-module $(K[\boldsymbol{x}] / I)_{P}$. We associate to $I$ the $K$-cycle

$$
X(I):=\sum_{P} m_{P} V(P) .
$$

If $V(I)$ is of pure dimension $r$, then $X(I) \in Z_{r}^{+}\left(\mathbb{P}_{K}^{n}\right)$. Let $K \subset E$ be an extension of fields and $V$ an irreducible $K$-variety. We define the scalar extension of $V$ by $E$ as the $E$-cycle $V_{E}=X\left(I(V) \otimes_{K} E\right)$. This notion extends to $K$-cycles by linearity and induces an inclusion of groups $Z_{r}\left(\mathbb{P}_{K}^{\boldsymbol{n}}\right) \hookrightarrow Z_{r}\left(\mathbb{P}_{E}^{\boldsymbol{n}}\right)$.

Each Weil or Cartier divisor of $\mathbb{P}_{K}^{n}$ is globally defined by a single rational multihomogeneous function in $K(\boldsymbol{x})$ because the ring $K[\boldsymbol{x}]$ is factorial [13, Prop. II.6.2 and II.6.11]. Hence, we will not make distinctions between them. We write $\operatorname{Div}\left(\mathbb{P}_{K}^{\boldsymbol{n}}\right)=Z_{|\boldsymbol{n}|-1}\left(\mathbb{P}_{K}^{\boldsymbol{n}}\right)$ for the group of divisors of $\mathbb{P}_{K}^{\boldsymbol{n}}$ and $\operatorname{Div}^{+}\left(\mathbb{P}_{K}^{\boldsymbol{n}}\right)=Z_{|\boldsymbol{n}|-1}^{+}\left(\mathbb{P}_{K}^{\boldsymbol{n}}\right)$ for the semigroup of those which are effective.

Each effective divisor $D$ of $\mathbb{P}_{K}^{\boldsymbol{n}}$ is defined by a multihomogeneous primitive polynomial in $A[\boldsymbol{x}] \backslash\{0\}$, unique up to a unit of $A$. We denote this polynomial by $f_{D}$. If we write 
$D=\sum_{H} m_{H} H$ where $H$ is a $K$-hypersurface of $\mathbb{P}^{\boldsymbol{n}}$ and $m_{H} \in \mathbb{N}$, then there exists $\lambda \in A^{\times}$ such that

$$
f_{D}=\lambda \prod_{H} f_{H}^{m_{H}}
$$

Conversely, given a multihomogeneous polynomial $f \in A[\boldsymbol{x}] \backslash\{0\}$, we denote by $\operatorname{div}(f) \in \operatorname{Div}^{+}\left(\mathbb{P}_{K}^{n}\right)$ the associated divisor.

We introduce some basic operations on cycles and divisors.

Definition 1.3. - Let $V$ be an irreducible subvariety of $\mathbb{P}_{K}^{n}$ and $H$ an irreducible hypersurface not containing $V$. Let $Y$ be an irreducible component of $V \cap H$. The intersection multiplicity of $V$ and $H$ along $Y$, denoted mult $(Y \mid V, H)$, is the length of the $K[\boldsymbol{x}]_{I(Y)}$-module $(K[\boldsymbol{x}] /(I(V)+I(H)))_{I(Y)}$, see [13, §I.1.7]. The intersection product of $V$ and $H$ is defined as

$$
V \cdot H=\sum_{Y} \operatorname{mult}(Y \mid V, H) Y,
$$

the sum being over the irreducible components of $V \cap H$. It is a cycle of pure dimen$\operatorname{sion} \operatorname{dim}(V)-1$.

Let $X$ be an equidimensional cycle and $D$ a divisor. We say that $X$ and $D$ intersect properly if no irreducible component of $X$ is contained in $|D|$. By bilinearity, the intersection product in (1.4) extends to a pairing

$$
Z_{r}\left(\mathbb{P}_{K}^{\boldsymbol{n}}\right) \times \operatorname{Div}\left(\mathbb{P}_{K}^{\boldsymbol{n}}\right) \rightarrow Z_{r-1}\left(\mathbb{P}_{K}^{\boldsymbol{n}}\right), \quad(X, D) \longmapsto X \cdot D,
$$

well-defined whenever $X$ and $D$ intersect properly.

Let $X \in Z_{r}\left(\mathbb{P}_{K}^{n}\right)$ and $D_{1}, \ldots, D_{\ell} \in \operatorname{Div}\left(\mathbb{P}_{K}^{n}\right)$. Then $X \cdot \prod_{j=1}^{\ell} D_{j}$ does not depend on the order of the divisors, provided that all the intermediate products are well-defined [11, Cor. 2.4.2 and Example 7.1.10(a)].

Definition 1.5. - Let $m_{1}, m_{2} \in \mathbb{Z}_{>0}$ and $\boldsymbol{n}_{i} \in \mathbb{N}^{m_{i}}$ for $i=1,2$. Let $\varphi: \mathbb{P}_{K}^{\boldsymbol{n}_{1}} \rightarrow \mathbb{P}_{K}^{\boldsymbol{n}_{2}}$ be a rational map and $V$ an irreducible subvariety of $\mathbb{P}_{K}^{n_{1}}$. The degree of $\varphi$ on $V$ is

$$
\operatorname{deg}\left(\left.\varphi\right|_{V}\right)=\left\{\begin{array}{cl}
{[K(V): K(\overline{\varphi(V)})]} & \text { if } \operatorname{dim}(\overline{\varphi(V)})=\operatorname{dim}(V), \\
0 & \text { if } \operatorname{dim}(\overline{\varphi(V)})<\operatorname{dim}(V) .
\end{array}\right.
$$

The direct image of $V$ under $\varphi$ is defined as the cycle $\varphi_{*} V=\operatorname{deg}\left(\left.\varphi\right|_{V}\right) \overline{\varphi(V)}$. It is a cycle of the same dimension as $V$. This notion extends by linearity to equidimensional cycles and induces a $\mathbb{Z}$-linear map

$$
\varphi_{*}: Z_{r}\left(\mathbb{P}_{K}^{\boldsymbol{n}_{1}}\right) \longrightarrow Z_{r}\left(\mathbb{P}_{K}^{\boldsymbol{n}_{2}}\right) .
$$

If $\psi: \mathbb{P}_{K}^{\boldsymbol{n}_{2}} \rightarrow \mathbb{P}_{K}^{\boldsymbol{n}_{3}}$ is a further rational map, then $(\psi \circ \varphi)_{*}=\psi_{*} \circ \varphi_{*}$ because of the multiplicativity of the degree of field extensions.

Let $H$ be a hypersuface of $\mathbb{P}_{K}^{n_{2}}$ not containing the image of $\varphi$. The inverse image of $H$ under $\varphi$ is defined as the hypersurface $\varphi^{*} H=\overline{\varphi^{-1}(H)}$. This notion extends to a $\mathbb{Z}$-linear map

$$
\varphi^{*}: \operatorname{Div}\left(\mathbb{P}_{K}^{\boldsymbol{n}_{2}}\right) \rightarrow \operatorname{Div}\left(\mathbb{P}_{K}^{\boldsymbol{n}_{1}}\right),
$$

well-defined for divisors whose support does not contain the image of $\varphi$.

4 e SÉRIE - TOME $46-2013$ - No 4 
Direct images of cycles, inverse images of divisors and intersection products are related by the projection formula [11, Prop. 2.3(c)]: let $\varphi: \mathbb{P}_{K}^{\boldsymbol{n}_{1}} \rightarrow \mathbb{P}_{K}^{\boldsymbol{n}_{2}}$ be a proper map, $X$ a cycle of $\mathbb{P}_{K}^{\boldsymbol{n}_{1}}$ and $D$ a divisor of $\mathbb{P}_{K}^{\boldsymbol{n}_{2}}$ containing no component of $\varphi(|X|)$. Then

$$
\varphi_{*}\left(X \cdot \varphi^{*} D\right)=\varphi_{*} X \cdot D \text {. }
$$

\subsection{Mixed degrees}

We recall the basic properties of mixed degrees of multiprojective cycles. We also study the behavior of this notion under linear projections.

Definition 1.7. - Let $V \subset \mathbb{P}_{K}^{n}$ be an irreducible $K$-variety. The Hilbert-Samuel function of $V$ is the numerical function defined as

$$
H_{V}: \mathbb{N}^{m} \longrightarrow \mathbb{N}, \quad \boldsymbol{\delta} \longmapsto \operatorname{dim}_{K}\left((K[\boldsymbol{x}] / I(V))_{\boldsymbol{\delta}}\right) .
$$

Proposition 1.8. - Let $V \subset \mathbb{P}_{K}^{n}$ be an irreducible $K$-variety of dimension $r$.

1. There is a unique polynomial $P_{V} \in \mathbb{Q}\left[z_{1}, \ldots, z_{m}\right]$ such that $P_{V}(\boldsymbol{\delta})=H_{V}(\boldsymbol{\delta})$ for all $\boldsymbol{\delta} \geq \boldsymbol{\delta}_{0}$ for some $\boldsymbol{\delta}_{0} \in \mathbb{N}^{m}$. In addition $\operatorname{deg}\left(P_{V}\right)=r$.

2. Let $\boldsymbol{b}=\left(b_{1}, \ldots, b_{m}\right) \in \mathbb{N}_{r}^{m}$. Then $\boldsymbol{b} ! \operatorname{coeff}_{\boldsymbol{b}}\left(P_{V}\right) \in \mathbb{N}$. Moreover, if $b_{i}>n_{i}$ for some $i$, then $\operatorname{coeff}_{\boldsymbol{b}}\left(P_{V}\right)=0$.

Proof. - (1) and the second part of (2) follow from [32, Thm. 2.10(1)]. The first part of (2) follows from [32, Thm. 2.10(2)] and its proof.

The polynomial $P_{V}$ in Proposition 1.8 is called the Hilbert-Samuel polynomial of $V$.

Definition 1.9. - Let $V \subset \mathbb{P}_{K}^{n}$ be an irreducible $K$-variety of dimension $r$ and $\boldsymbol{b} \in \mathbb{N}_{r}^{m}$. The (mixed) degree of $V$ of index $\boldsymbol{b}$ is defined as

$$
\operatorname{deg}_{\boldsymbol{b}}(V)=\boldsymbol{b} ! \operatorname{coeff}_{\boldsymbol{b}}\left(P_{V}\right) .
$$

It is a non-negative integer, thanks to Proposition 1.8(2). This notion extends by linearity to

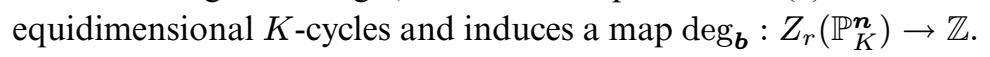

Recall that the Chow ring of $\mathbb{P}_{K}^{n}$ is the graded ring

$$
A^{*}\left(\mathbb{P}_{K}^{\boldsymbol{n}}\right)=\mathbb{Z}\left[\theta_{1}, \ldots, \theta_{m}\right] /\left(\theta_{1}^{n_{1}+1}, \ldots, \theta_{m}^{n_{m}+1}\right),
$$

where each $\theta_{i}$ denotes the class of the inverse image of a hyperplane of $\mathbb{P}_{K}^{n_{i}}$ under the projection $\mathbb{P}_{K}^{n} \rightarrow \mathbb{P}_{K}^{n_{i}}\left[11\right.$, Example 8.3.7]. Given a cycle $X \in Z_{r}\left(\mathbb{P}_{K}^{\boldsymbol{n}}\right)$, its class in the Chow ring is

$$
[X]=\sum_{\boldsymbol{b}} \operatorname{deg}_{\boldsymbol{b}}(X) \theta_{1}^{n_{1}-b_{1}} \cdots \theta_{m}^{n_{m}-b_{m}} \in A^{*}\left(\mathbb{P}_{K}^{\boldsymbol{n}}\right),
$$

the sum being over all $\boldsymbol{b} \in \mathbb{N}_{r}^{m}$ such that $\boldsymbol{b} \leq \boldsymbol{n}$. It is a homogeneous element of degree $|\boldsymbol{n}|-r$. By Proposition 1.8(2), $\operatorname{deg}_{\boldsymbol{b}}(X)=0$ whenever $b_{i}>n_{i}$ for some $i$. Hence, $[X]$ contains the information of all the mixed degrees of $X$, since $\left\{\boldsymbol{\theta}^{\boldsymbol{b}}\right\}_{\boldsymbol{b} \leq \boldsymbol{n}}$ is a $\mathbb{Z}$-basis of $\mathbb{A}^{*}\left(\mathbb{P}_{K}^{\boldsymbol{n}}\right)$. For $X_{1}, X_{2} \in Z_{r}\left(\mathbb{P}_{K}^{n}\right)$, we say that $\left[X_{1}\right] \geq\left[X_{2}\right]$ whenever the inequality holds coefficientwise in terms of this basis.

Given a $K$-cycle $X$, its class in the Chow ring is invariant under field extensions. In particular, $\left[X_{\bar{K}}\right]=[X]$ and $\operatorname{deg}_{\boldsymbol{b}}(X)=\operatorname{deg}_{\boldsymbol{b}}\left(X_{\bar{K}}\right)$ for all $\boldsymbol{b} \in \mathbb{N}_{r}^{m}$. If $\operatorname{dim}(X)=0$, its degree is defined as the number of points in $X_{\bar{K}}$, counted with multiplicity. 
Proposition 1.10. - $\quad$ 1. Let $X \in Z_{r}^{+}\left(\mathbb{P}_{K}^{\boldsymbol{n}}\right)$. Then $[X] \geq 0$.

2. We have $\left[\mathbb{P}_{K}^{\boldsymbol{n}}\right]=1$. Equivalently, $\operatorname{deg}_{\boldsymbol{n}}\left(\mathbb{P}_{K}^{\boldsymbol{n}}\right)=1$ and $\operatorname{deg}_{\boldsymbol{b}}\left(\mathbb{P}_{K}^{\boldsymbol{n}}\right)=0$ for all $\boldsymbol{b} \in \mathbb{N}_{|\boldsymbol{n}|}^{m}$ such that $\boldsymbol{b} \neq \boldsymbol{n}$.

3. Let $X \in Z_{0}\left(\mathbb{P}_{K}^{\boldsymbol{n}}\right)$. Then $[X]=\operatorname{deg}(X) \boldsymbol{\theta}^{\boldsymbol{n}}$. Equivalently, $\operatorname{deg}_{\mathbf{0}}(X)=\operatorname{deg}(X)$.

4. Let $D \in \operatorname{Div}^{+}\left(\mathbb{P}_{K}^{n}\right)$ and $f_{D}$ its defining polynomial. Then

$$
[D]=\sum_{i=1}^{m} \operatorname{deg}_{\boldsymbol{x}_{i}}\left(f_{D}\right) \theta_{i}
$$

Equivalently, $\operatorname{deg}_{\boldsymbol{n}-\boldsymbol{e}_{i}}(D)=\operatorname{deg}_{\boldsymbol{x}_{i}}\left(f_{D}\right)$ for $1 \leq i \leq m$ and $\operatorname{deg}_{\boldsymbol{b}}(D)=0$ for all $\boldsymbol{b} \in \mathbb{N}_{|\boldsymbol{n}|-1}^{m}$ such that $\boldsymbol{b} \neq \boldsymbol{n}-\boldsymbol{e}_{i}$ for all $i$.

5. Let $n \in \mathbb{N}$ and $V \subset \mathbb{P}_{K}^{n}$ a $K$-variety of pure dimension $r$. Then

$$
[V]=\operatorname{deg}(V) \theta^{n-r},
$$

where $\operatorname{deg}(V)$ denotes the degree of the projective variety $V$. Equivalently, $\operatorname{deg}_{r}(V)=\operatorname{deg}(V)$.

Proof. - (1) This follows from the definition of $[X]$ and Proposition 1.8(2).

(2) For $\boldsymbol{\delta}=\left(\delta_{1}, \ldots, \delta_{m}\right) \in \mathbb{N}^{m}$,

$$
H_{\mathbb{P}_{K}^{n}}(\boldsymbol{\delta})=\operatorname{dim}_{K}\left(K[\boldsymbol{x}]_{\boldsymbol{\delta}}\right)=\prod_{i=1}^{m}\left(\begin{array}{c}
n_{i}+\delta_{i} \\
n_{i}
\end{array}\right)=\frac{1}{n_{1} ! \cdots n_{m} !} \boldsymbol{\delta}^{\boldsymbol{n}}+O\left(\|\boldsymbol{\delta}\|^{|\boldsymbol{n}|-1}\right),
$$

where $\|\cdot\|$ denotes any fixed norm on $\mathbb{R}^{m}$. This implies that $\operatorname{deg}_{\boldsymbol{n}}\left(\mathbb{P}_{K}^{\boldsymbol{n}}\right)=1$ and thus $\left[\mathbb{P}_{K}^{\boldsymbol{n}}\right]=\operatorname{deg}_{\boldsymbol{n}}\left(\mathbb{P}_{K}^{\boldsymbol{n}}\right)=1$, as stated.

(3) Let $\boldsymbol{\xi}=\left(\boldsymbol{\xi}_{1}, \ldots, \boldsymbol{\xi}_{m}\right) \in \mathbb{P}^{\boldsymbol{n}}$. We have $\bar{K}[\boldsymbol{x}] / I(\boldsymbol{\xi})=\bigotimes_{i=1}^{m} \bar{K}\left[\boldsymbol{x}_{i}\right] / I\left(\boldsymbol{\xi}_{i}\right)$. Hence, for $\boldsymbol{\delta} \in \mathbb{N}^{m}$,

$$
H_{\boldsymbol{\xi}}(\boldsymbol{\delta})=\operatorname{dim}_{\bar{K}}\left((\bar{K}[\boldsymbol{x}] / I(\boldsymbol{\xi}))_{\boldsymbol{\delta}}\right)=\prod_{i=1}^{m} \operatorname{dim}_{\bar{K}}\left(\bar{K}\left[\boldsymbol{x}_{i}\right] / I\left(\boldsymbol{\xi}_{i}\right)\right)_{\delta_{i}}=1 .
$$

This implies that $\operatorname{deg}_{\mathbf{0}}(\boldsymbol{\xi})=1$ and so $[\boldsymbol{\xi}]=\boldsymbol{\theta}^{\boldsymbol{n}}$. For a general zero-dimensional $K$-cycle $X$, write $X_{\bar{K}}=\sum_{\boldsymbol{\xi}} m_{\boldsymbol{\xi}} \boldsymbol{\xi}$ for some points $\boldsymbol{\xi} \in \mathbb{P}^{\boldsymbol{n}}$ and $m_{\boldsymbol{\xi}} \in \mathbb{Z}$. Hence, $\operatorname{deg}_{\mathbf{0}}(X)=$ $\sum_{\boldsymbol{\xi}} m_{\boldsymbol{\xi}} \operatorname{deg}_{\mathbf{0}}(\boldsymbol{\xi})=\sum_{\boldsymbol{\xi}} m_{\boldsymbol{\xi}}=\operatorname{deg}(X)$ and so $[X]=\operatorname{deg}(X) \boldsymbol{\theta}^{\boldsymbol{n}}$.

(4) Write $\operatorname{deg}(f)=\left(\operatorname{deg}_{\boldsymbol{x}_{1}}(f), \ldots, \operatorname{deg}_{\boldsymbol{x}_{m}}(f)\right)$. For $\boldsymbol{\delta} \geq \operatorname{deg}(f)$, there is an exact sequence

$$
0 \longrightarrow K[\boldsymbol{x}]_{\boldsymbol{\delta}-\operatorname{deg}(f)} \stackrel{\times f}{\longrightarrow} K[\boldsymbol{x}]_{\boldsymbol{\delta}} \longrightarrow(K[\boldsymbol{x}] /(f))_{\boldsymbol{\delta}} \longrightarrow 0 .
$$

Hence, $H_{D}(\boldsymbol{\delta})=H_{\mathbb{P}_{K}^{n}}(\boldsymbol{\delta})-H_{\mathbb{P}_{K}^{n}}\left(\boldsymbol{\delta}-\operatorname{deg}\left(f_{D}\right)\right)$ and therefore

$$
P_{D}(\boldsymbol{d})=\sum_{i=1}^{m} \frac{\operatorname{deg}_{\boldsymbol{x}_{i}}(f)}{\left(\boldsymbol{n}-\boldsymbol{e}_{i}\right) !} \boldsymbol{\delta}^{\boldsymbol{n}-\boldsymbol{e}_{i}}+O\left(\|\boldsymbol{\delta}\|^{|\boldsymbol{n}|-2}\right) .
$$

This implies that $\operatorname{deg}_{\boldsymbol{n}-\boldsymbol{e}_{i}}(D)=\operatorname{deg}_{\boldsymbol{x}_{i}}(f)$ and so $[D]=\sum_{i=1}^{m} \operatorname{deg}_{\boldsymbol{x}_{i}}\left(f_{D}\right) \theta_{i}$, as stated.

(5) This follows readily from the definition of $\operatorname{deg}(V)$ in terms of Hilbert functions.

The following is the multiprojective version of Bézout's theorem. 
Theorem 1.11. - Let $X \in Z_{r}\left(\mathbb{P}_{K}^{n}\right)$ and $f \in K\left[\boldsymbol{x}_{1}, \ldots, \boldsymbol{x}_{m}\right]$ be a multihomogeneous polynomial such that $X$ and $\operatorname{div}(f)$ intersect properly. Then

$$
[X \cdot \operatorname{div}(f)]=[X] \cdot[\operatorname{div}(f)] .
$$

Equivalently, $\operatorname{deg}_{\boldsymbol{b}}(X \cdot \operatorname{div}(f))=\sum_{i=1}^{m} \operatorname{deg}_{\boldsymbol{x}_{i}}(f) \operatorname{deg}_{\boldsymbol{b}+\boldsymbol{e}_{i}}(X)$ for all $\boldsymbol{b} \in \mathbb{N}_{r-1}^{m}$.

Proof. - The equivalence between the two statements follows from Proposition 1.10(4). The second statement follows for instance from [33, Thm. 3.4].

Next corollary follows readily from this result together with Proposition 1.10(3).

Corollary 1.12. - Let $f_{1}, \ldots, f_{|\boldsymbol{n}|} \in K\left[\boldsymbol{x}_{1}, \ldots, \boldsymbol{x}_{m}\right]$ be multihomogeneous polynomials such that $\operatorname{dim}\left(V\left(f_{1}, \ldots, f_{i}\right)\right)=|\boldsymbol{n}|-i$ for all $i$. Then

$$
\operatorname{deg}\left(\prod_{i=1}^{|\boldsymbol{n}|} \operatorname{div}\left(f_{i}\right)\right)=\operatorname{coeff}_{\boldsymbol{\theta}^{n}}\left(\prod_{i=1}^{|\boldsymbol{n}|}\left(\operatorname{deg}_{\boldsymbol{x}_{1}}\left(f_{i}\right) \theta_{1}+\cdots+\operatorname{deg}_{\boldsymbol{x}_{m}}\left(f_{i}\right) \theta_{m}\right)\right) .
$$

EXAMPLE 1.13. - How many pairs (eigenvalue, eigenvector) can a generic square matrix have? Given $M=\left(m_{i, j}\right)_{i, j} \in K^{n \times n}$, the problem of computing these pairs consists in solving $M \boldsymbol{v}=\lambda \boldsymbol{v}$ for $\lambda \in \bar{K}$ and $\boldsymbol{v}=\left(v_{1}, \ldots, v_{n}\right) \in \bar{K}^{n} \backslash\{0\}$. Set

$$
f_{i}=s_{1} v_{i}-s_{0} \sum_{j=1}^{n} m_{i, j} v_{j}, \quad 1 \leq i \leq n .
$$

The matrix equation $M \boldsymbol{v}=\lambda \boldsymbol{v}$ translates into the system of $n$ bilinear scalar equations $f_{i}=0,1 \leq i \leq n$, for $\left(\left(s_{0}: s_{1}\right), \boldsymbol{v}\right) \in \mathbb{P}^{1} \times \mathbb{P}^{n-1}$ such that $s_{0} \neq 0$. If $M$ is generic, the hypersurfaces $V\left(f_{i}\right)$ intersect properly. By Corollary 1.12, the number of solutions in $\mathbb{P}^{1} \times \mathbb{P}^{n-1}$ of this system of equations is

$$
\operatorname{coeff}_{\theta_{1} \theta_{2}^{n-1}}\left(\prod_{i=1}^{n} \operatorname{deg}_{\boldsymbol{s}}\left(f_{i}\right) \theta_{1}+\operatorname{deg}_{\boldsymbol{v}}\left(f_{i}\right) \theta_{2}\right)=\operatorname{coeff}_{\theta_{1} \theta_{2}^{n-1}}\left(\left(\theta_{1}+\theta_{2}\right)^{n}\right)=n .
$$

We deduce that $M$ admits at most $n$ pairs (eigenvalue, eigenvector) counted with multiplicities. A straightforward application of the usual Bézout's theorem would have given the much larger bound $2^{n}$.

The following result shows that mixed degrees can also be defined geometrically.

Corollary 1.14. - Let $X \in Z_{r}\left(\mathbb{P}_{K}^{\boldsymbol{n}}\right)$ and $\boldsymbol{b} \in \mathbb{N}_{r}^{m}$. For $1 \leq i \leq m$ and $0 \leq j \leq b_{i}$ we denote by $H_{i, j} \subset \mathbb{P}_{K}^{n}$ the inverse image with respect to the projection $\mathbb{P}_{K}^{n} \rightarrow \mathbb{P}_{K}^{n_{i}}$ of a generic hyperplane of $\mathbb{P}_{K}^{n_{i}}$. Then

$$
\operatorname{deg}_{\boldsymbol{b}}(X)=\operatorname{deg}\left(X \cdot \prod_{i=1}^{m} \prod_{j=1}^{b_{i}} H_{i, j}\right)
$$


Proof. - The variety $X$ and the divisors $H_{i, j}$ intersect properly and $\left[H_{i, j}\right]=\theta_{i}$. Theorem 1.11 implies that, for $Z=X \cdot \prod_{i=1}^{m} \prod_{j=1}^{b_{i}} H_{i, j} \in Z_{0}\left(\mathbb{P}_{K}^{\boldsymbol{n}}\right)$,

$$
\operatorname{deg}(Z) \boldsymbol{\theta}^{\boldsymbol{n}}=[Z]=[X] \cdot \prod_{i=1}^{m} \prod_{j=1}^{b_{i}}\left[H_{i, j}\right]=[X] \boldsymbol{\theta}^{\boldsymbol{b}}=\left(\sum_{\boldsymbol{c} \in \mathbb{N}_{r}^{m}} \operatorname{deg}_{\boldsymbol{c}}(X) \boldsymbol{\theta}^{\boldsymbol{n}-\boldsymbol{c}}\right) \boldsymbol{\theta}^{\boldsymbol{b}}=\operatorname{deg}_{\boldsymbol{b}}(X) \boldsymbol{\theta}^{\boldsymbol{n}}
$$

which proves the statement.

Next we show that mixed degrees are monotonic with respect to linear projections. For $1 \leq i \leq m$, let $0 \leq l_{i} \leq n_{i}$ and set $\boldsymbol{l}=\left(l_{1}, \ldots, l_{m}\right) \in \mathbb{N}^{m}$. Consider the linear projection which forgets the last $n_{i}-l_{i}$ coordinates in each factor of $\mathbb{P}_{K}^{n}$ :

$$
\pi: \mathbb{P}_{K}^{\boldsymbol{n}} \rightarrow \mathbb{P}_{K}^{\boldsymbol{l}}, \quad\left(x_{i, j}\right)_{\substack{1 \leq i \leq m \\ 0 \leq j \leq n_{i}}} \longmapsto\left(x_{i, j}\right)_{\substack{1 \leq i \leq m \\ 0 \leq j \leq l_{i}}}
$$

This is a rational map, well-defined outside the union of linear subspaces $L:=\bigcup_{i=1}^{m} V\left(x_{i, 0}, \ldots, x_{i, l_{i}}\right) \subset \mathbb{P}_{K}^{n}$. It induces an injective $\mathbb{Z}$-linear map

$$
\jmath: A^{*}\left(\mathbb{P}_{K}^{l}\right) \longleftrightarrow A^{*}\left(\mathbb{P}_{K}^{n}\right), \quad P \longmapsto \boldsymbol{\theta}^{n-l} P .
$$

Proposition 1.16. - Let $\pi: \mathbb{P}_{K}^{n} \rightarrow \mathbb{P}_{K}^{l}$ be the linear projection as above and $X \in Z_{r}^{+}\left(\mathbb{P}_{K}^{\boldsymbol{n}}\right)$. Then

$$
\jmath\left(\left[\pi_{*} X\right]\right) \leq[X]
$$

Equivalently, $\operatorname{deg}_{\boldsymbol{b}}\left(\pi_{*} X\right) \leq \operatorname{deg}_{\boldsymbol{b}}(X)$ for all $\boldsymbol{b} \in \mathbb{N}_{r}^{m}$.

The proof of this result relies on the technical Lemma 1.18 below, which was suggested to us by José Ignacio Burgos. Consider the blow-up of $\mathbb{P}^{n}$ along the subvariety $L$, denoted $\mathrm{Bl}_{L}\left(\mathbb{P}^{n}\right)$ and defined as the closure in $\mathbb{P}^{n} \times \mathbb{P}^{l}$ of the graph of $\pi$. It is an irreducible variety of dimension $|\boldsymbol{n}|$. Set $\boldsymbol{x}$ and $\boldsymbol{y}$ for the multihomogeneous coordinates of $\mathbb{P}^{\boldsymbol{n}}$ and $\mathbb{P}^{\boldsymbol{l}}$, respectively. The ideal of this variety is

$$
I\left(\mathrm{Bl}_{L}\left(\mathbb{P}^{\boldsymbol{n}}\right)\right)=\left(\left\{x_{i, j_{1}} y_{i, j_{2}}-x_{i, j_{2}} y_{i, j_{1}}: 1 \leq i \leq m, 0 \leq j_{1}<j_{2} \leq l_{i}\right\}\right) \subset \bar{K}[\boldsymbol{x}, \boldsymbol{y}] .
$$

Consider the projections

$$
\operatorname{pr}_{1}: \mathbb{P}^{n} \times \mathbb{P}^{l} \longrightarrow \mathbb{P}^{n}, \quad \operatorname{pr}_{2}: \mathbb{P}^{n} \times \mathbb{P}^{l} \longrightarrow \mathbb{P}^{l}
$$

The exceptional divisor of the blow-up is supported in the hypersurface $E=\operatorname{pr}_{1}^{-1}(L)$. Let $V \subset \mathbb{P}^{n}$ be an irreducible variety such that $V \not \subset L$ and $W$ its strict transform, which is the closure of the set $\operatorname{pr}_{1}^{-1}(V \backslash L) \cap \mathrm{Bl}_{L}\left(\mathbb{P}^{n}\right)$. Then

$$
\operatorname{pr}_{1 *} W=V, \quad \operatorname{pr}_{2 *} W=\pi_{*} V .
$$

For a multihomogeneous polynomial $f \in \bar{K}[\boldsymbol{y}] \backslash\{0\}$, we write $\operatorname{div}_{\mathbb{P}^{l}}(f)$ for the divisor of $\mathbb{P}^{\boldsymbol{l}}$ defined by $f(\boldsymbol{y})$ and $\operatorname{div}_{\mathbb{P} n}(f)$ for the divisor of $\mathbb{P}^{n}$ defined by $f\left(\left(x_{i, j}\right)_{\substack{1 \leq i \leq m \\ 0 \leq j \leq \ell_{i}}}\right)$.

Lemma 1.18. - Let $V \subset \mathbb{P}^{n}$ be an irreducible variety of dimension $r$ such that $V \not \subset L$ and $f \in \bar{K}[\boldsymbol{y}] \backslash\{0\}$ a multihomogeneous polynomial. Assume that $\operatorname{pr}_{2}^{*} \operatorname{div}_{\mathbb{P}^{l}}(f)$ intersects $W$ properly and that no component of $W \cdot \operatorname{pr}_{2}^{*} \operatorname{div}_{\mathbb{P}^{l}}(f)$ is contained in $E$. Then $\operatorname{div}_{\mathbb{P}^{l}}(f)$ (respectively, $\operatorname{div}_{\mathbb{P} n}(f)$ ) intersects $\pi_{*} V$ (respectively, $V$ ) properly and

$$
\pi_{*}\left(V \cdot \operatorname{div}_{\mathbb{P} n}(f)\right)=\pi_{*} V \cdot \operatorname{div}_{\mathbb{P}^{l}}(f) .
$$

$4^{\mathrm{e}}$ SÉRIE - TOME $46-2013-\mathrm{N}^{\circ} 4$ 
Proof. - Consider the following divisors of $\mathbb{P}^{n} \times \mathbb{P}^{l}$ :

$$
D_{1}=\operatorname{pr}_{1}^{*} \operatorname{div}_{\mathbb{P} n}(f), \quad D_{2}=\operatorname{pr}_{2}^{*} \operatorname{div}_{\mathbb{P} l}(f) .
$$

Write for short $B=\mathrm{Bl}_{L}\left(\mathbb{P}^{n}\right)$. Since $W$ is irreducible, the hypothesis that $D_{2}$ intersects $W$ properly is equivalent to the fact that $W$ is not contained in $\left|D_{2}\right|$. This implies that neither $V$ is contained in $V(f(\boldsymbol{x}))$ nor $\pi(V)$ is contained in $V(f(\boldsymbol{y}))$. Hence all intersection products are well-defined. We claim that

$$
B \cdot\left(D_{2}-D_{1}\right)
$$

is a cycle of pure dimension $|\boldsymbol{n}|-1$ with support contained in the hypersurface $E$. To prove this, write $d_{i}=\operatorname{deg}_{\boldsymbol{x}_{i}}(f)$ and, for each $1 \leq i \leq m$, choose an index $0 \leq j_{i} \leq l_{i}$. Using (1.17) we verify that

$$
\left(\prod_{i=1}^{m} y_{i, j_{i}}^{d_{i}}\right) f(\boldsymbol{x}) \equiv\left(\prod_{i=1}^{m} x_{i, j_{i}}^{d_{i}}\right) f(\boldsymbol{y}) \quad(\bmod I(B)) .
$$

Observe that the ideal of $E$ in $\bar{K}[\boldsymbol{x}, \boldsymbol{y}] / I(B)$ is generated by the set of monomials

$$
\left(\prod_{i=1}^{m} x_{i, j_{i}}\right)_{\substack{1 \leq i \leq m \\ 0 \leq j_{i} \leq l_{i}}} .
$$

Let $\left(\boldsymbol{\xi}, \boldsymbol{\xi}^{\prime}\right) \in B \backslash E$. We have that $\prod_{i=1}^{m} \xi_{i, j_{i}} \neq 0$ for a choice of $j_{i}$ 's. From here, we can verify that $\prod_{i=1}^{m} \xi_{i, j_{i}}^{\prime} \neq 0$. This implies that $f(\boldsymbol{x})$ and $f(\boldsymbol{y})$ generate the same ideal in the localization $(\bar{K}[\boldsymbol{x}, \boldsymbol{y}] / I(B))_{\left(\boldsymbol{\xi}, \boldsymbol{\xi}^{\prime}\right)}$ for all such $\left(\boldsymbol{\xi}, \boldsymbol{\xi}^{\prime}\right)$ and proves the claim. Therefore, there exists a cycle $Z \in Z_{r-1}\left(\mathbb{P}^{n} \times \mathbb{P}^{l}\right)$ supported on $E$ such that

$$
W \cdot D_{1}=W \cdot D_{2}-Z \text {. }
$$

Since the map $\mathrm{pr}_{1}$ is proper, the projection Formula (1.6) implies that

$$
V \cdot \operatorname{div}_{\mathbb{P} n}(f)=\operatorname{pr}_{1 *}\left(W \cdot D_{1}\right)=\operatorname{pr}_{1 *}\left(W \cdot D_{2}\right)-\operatorname{pr}_{1 *} Z
$$

By hypothesis, no component of $W \cdot D_{2}$ is contained in $E$. Since $\operatorname{pr}_{1}: B \backslash E \rightarrow \mathbb{P}^{\boldsymbol{n}} \backslash L$ is an isomorphism, no component of $\operatorname{pr}_{1 *}\left(W \cdot D_{2}\right)$ is contained in $L$. Hence,

$$
\left.\pi_{*}\left(V \cdot \operatorname{div}_{\mathbb{P}^{n}}(f)\right)\right)=\pi_{*} \operatorname{pr}_{1 *}\left(W \cdot D_{2}\right)-\pi_{*} \operatorname{pr}_{1 *} Z=\left(\pi \circ \operatorname{pr}_{1}\right)_{*}\left(W \cdot D_{2}\right)=\operatorname{pr}_{2 *}\left(W \cdot D_{2}\right),
$$

because $\pi_{*} \operatorname{pr}_{1 *} Z=0$ as this is a cycle supported on $L$. Again by the projection formula,

$$
\operatorname{pr}_{2 *}\left(W \cdot D_{2}\right)=\operatorname{pr}_{2 *} W \cdot \operatorname{div}_{\mathbb{P}^{l}}(f)=\pi_{*} V \cdot \operatorname{div}_{\mathbb{P}}(f),
$$

which proves the statement.

Proof of Proposition 1.16. - The equivalence between the two formulations is a direct consequence of the definitions. We reduce without loss of generality to the case of an irreducible variety $V \subset \mathbb{P}^{\boldsymbol{n}}$ such that $\operatorname{dim}(\overline{\pi(V)})=r$.

We proceed by induction on the dimension. For $r=0$, the statement is obvious and so we assume $r \geq 1$. Let $1 \leq i \leq m$ such that $b_{i} \neq 0$ and $\ell \in \bar{K}\left[\boldsymbol{y}_{i}\right]$ a linear form. For each component $C$ of $W \cap E$ we pick a point $\boldsymbol{\xi}_{C} \in C$ and we impose that $\ell\left(\boldsymbol{\xi}_{C}\right) \neq 0$, which holds 
for a generic choice of $\ell$. This implies that $\operatorname{pr}_{2}^{*}\left(\operatorname{div}_{\mathbb{P} l}(\ell)\right)$ intersects $W$ properly and that their intersection has no component contained in $E$. Hence

$$
\begin{aligned}
\operatorname{deg}_{\boldsymbol{b}}\left(\pi_{*} V\right) & =\operatorname{deg}_{\boldsymbol{b}-\boldsymbol{e}_{i}}\left(\pi_{*} V \cdot \operatorname{div}_{\mathbb{P} l}(\ell)\right) & & \text { by Bézout's Theorem 1.11, } \\
& =\operatorname{deg}_{\boldsymbol{b}-\boldsymbol{e}_{i}}\left(\pi_{*}\left(V \cdot \operatorname{div}_{\mathbb{P} \boldsymbol{n}}(\ell)\right)\right) & & \text { by Lemma 1.18, } \\
& \left.\leq \operatorname{deg}_{\boldsymbol{b}-\boldsymbol{e}_{i}}\left(V \cdot \operatorname{div}_{\mathbb{P} \boldsymbol{n}}(\ell)\right)\right) & & \text { by the inductive hypothesis, } \\
& =\operatorname{deg}_{\boldsymbol{b}}(V) & & \text { by Bézout's Theorem 1.11, }
\end{aligned}
$$

which completes the proof.

Next result gives the behavior of Chow rings and classes with respect to products.

Proposition 1.19. - Let $m_{i} \in \mathbb{Z}_{>0}$ and $\boldsymbol{n}_{i} \in \mathbb{N}^{m_{i}}$ for $i=1,2$. Then

1. $A^{*}\left(\mathbb{P}_{K}^{\boldsymbol{n}_{1}} \times \mathbb{P}_{K}^{\boldsymbol{n}_{2}}\right) \simeq A^{*}\left(\mathbb{P}_{K}^{\boldsymbol{n}_{1}}\right) \otimes_{\mathbb{Z}} A^{*}\left(\mathbb{P}_{K}^{\boldsymbol{n}_{2}}\right)$.

2. Let $X_{i} \in Z_{r_{i}}\left(\mathbb{P}_{K}^{n_{i}}\right)$ for $i=1,2$. The above isomorphism identifies $\left[X_{1} \times X_{2}\right]$ with $\left[X_{1}\right] \otimes_{\mathbb{Z}}\left[X_{2}\right]$. Equivalently, for all $\boldsymbol{b}_{i} \in \mathbb{N}^{m_{i}}$ such that $\left|\boldsymbol{b}_{1}\right|+\left|\boldsymbol{b}_{2}\right|=r_{1}+r_{2}$,

$$
\operatorname{deg}_{\boldsymbol{b}_{1}, \boldsymbol{b}_{2}}\left(X_{1} \times X_{2}\right)=\left\{\begin{array}{cl}
\operatorname{deg}_{\boldsymbol{b}_{1}}\left(X_{1}\right) \operatorname{deg}_{\boldsymbol{b}_{2}}\left(X_{2}\right) & \text { if }\left|\boldsymbol{b}_{1}\right|=r_{1},\left|\boldsymbol{b}_{2}\right|=r_{2}, \\
0 & \text { otherwise. }
\end{array}\right.
$$

Proof. - (1) This is immediate from the definition of the Chow ring.

(2) We reduce without loss of generality to the case of irreducible $K$-varieties $V_{i} \subset \mathbb{P}_{K}^{\boldsymbol{n}_{i}}$, $i=1,2$. Let $\boldsymbol{x}_{i}$ denote the multihomogeneous coordinates of $\mathbb{P}_{K}^{\boldsymbol{n}_{i}}$. For $\boldsymbol{\delta}_{i} \in \mathbb{N}^{m_{i}}$,

$$
\left(K\left[\boldsymbol{x}_{1}, \boldsymbol{x}_{2}\right] / I\left(V_{1} \times V_{2}\right)\right)_{\boldsymbol{\delta}_{1}, \boldsymbol{\delta}_{2}} \simeq\left(K\left[\boldsymbol{x}_{1}\right] / I\left(V_{1}\right)\right)_{\boldsymbol{\delta}_{1}} \otimes\left(K\left[\boldsymbol{x}_{2}\right] / I\left(V_{2}\right)\right)_{\boldsymbol{\delta}_{2}} .
$$

Hence $H_{V_{1} \times V_{2}}\left(\boldsymbol{\delta}_{1}, \boldsymbol{\delta}_{2}\right)=H_{V_{1}}\left(\boldsymbol{\delta}_{1}\right) H_{V_{2}}\left(\boldsymbol{\delta}_{2}\right)$ and therefore $P_{V_{1} \times V_{2}}=P_{V_{1}} P_{V_{2}}$. This implies the equality of mixed degrees, which in turn implies that $\left[V_{1} \times V_{2}\right]=\left[V_{1}\right] \otimes\left[V_{2}\right]$ under the identification in (1).

We end this section with the intersection-theoretic properties of the ruled join of projective varieties. Let $n_{i} \in \mathbb{N}$ and consider an irreducible $K$-variety $V_{i} \subset \mathbb{P}_{K}^{n_{i}}$ for $i=1,2$. Let $K\left[\boldsymbol{x}_{i}\right]$ denote the homogeneous coordinate ring of $\mathbb{P}_{K}^{n_{i}}$ and $I\left(V_{i}\right) \subset K\left[\boldsymbol{x}_{i}\right]$ the ideal of $V_{i}$. The ruled join of $V_{1}$ and $V_{2}$, denoted by $V_{1} \# V_{2}$, is the irreducible subvariety of $\mathbb{P}_{K}^{n_{1}+n_{2}+1}$ defined by the homogeneous ideal generated by $I\left(V_{1}\right) \cup I\left(V_{2}\right)$ in $K\left[\boldsymbol{x}_{1}, \boldsymbol{x}_{2}\right]$. In case $K$ is algebraically closed, identifying $\mathbb{P}^{n_{1}}$ and $\mathbb{P}^{n_{2}}$ with the linear subspaces of $\mathbb{P}^{n_{1}+n_{2}+1}$ where the last $n_{2}+1$ (respectively, the first $n_{1}+1$ ) coordinates vanish, $V_{1} \# V_{2}$ coincides with the union of the lines of $\mathbb{P}^{n_{1}+n_{2}+1}$ joining points of $V_{1}$ with points of $V_{2}$.

The notion of ruled join extends to equidimensional $K$-cycles by linearity. Given cycles $X_{i} \in Z_{r_{i}}\left(\mathbb{P}_{K}^{n_{i}}\right), i=1,2$, the ruled join $X_{1} \# X_{2}$ is a cycle of $\mathbb{P}_{K}^{n_{1}+n_{2}+1}$ of pure dimension $r_{1}+r_{2}+1$ and degree

$$
\operatorname{deg}\left(X_{1} \# X_{2}\right)=\operatorname{deg}\left(X_{1}\right) \operatorname{deg}\left(X_{2}\right),
$$

see for instance [11, Example 8.4.5]. For $i=1,2$ consider the injective $\mathbb{Z}$-linear map $\jmath_{i}: A^{*}\left(\mathbb{P}_{K}^{n_{i}}\right) \hookrightarrow A^{*}\left(\mathbb{P}_{K}^{n_{1}+n_{2}+1}\right)$ defined by $\theta^{l} \mapsto \theta^{l}$ for $0 \leq l \leq n_{i}$. Then (1.20) is equivalent to the equality of classes

$$
\left[X_{1} \# X_{2}\right]=\jmath_{1}\left(\left[X_{1}\right]\right) \cdot \jmath_{2}\left(\left[X_{2}\right]\right) .
$$




\subsection{Eliminants and resultants}

In this section, we introduce the notions and basic properties of eliminants of varieties and of resultants of cycles in multiprojective spaces. This is mostly a reformulation of the theory of eliminants and resultants of multihomogeneous ideals developed by Rémond in [32, 33] as an extension of Philippon's theory of eliminants of homogeneous ideals [27]. We refer the reader to these articles for a complementary presentation of the subject.

We keep the notation of $\S 1$.1. In particular, we denote by $A$ a factorial ring with field of fractions $K$. Let $V \subset \mathbb{P}_{K}^{n}$ be an irreducible $K$-variety of dimension $r$. Let $\boldsymbol{d}_{0}, \ldots, \boldsymbol{d}_{r} \in \mathbb{N}^{m} \backslash\{\boldsymbol{0}\}$ and set $\boldsymbol{d}=\left(\boldsymbol{d}_{0}, \ldots, \boldsymbol{d}_{r}\right)$. For each $0 \leq i \leq r$, we introduce a group of variables $\boldsymbol{u}_{i}=\left\{u_{i, \boldsymbol{a}}: \boldsymbol{a} \in \mathbb{N}_{\boldsymbol{d}_{i}}^{\boldsymbol{n}+\mathbf{1}}\right\}$ and consider the general form $F_{i}$ of multidegree $\boldsymbol{d}_{i}$ in the variables $\boldsymbol{x}$ :

$$
F_{i}=\sum_{\boldsymbol{a} \in \mathbb{N}_{\boldsymbol{d}_{i}}^{n+1}} u_{i, \boldsymbol{a}} \boldsymbol{x}^{\boldsymbol{a}} \in K\left[\boldsymbol{u}_{i}\right][\boldsymbol{x}] .
$$

Set $\boldsymbol{u}=\left\{\boldsymbol{u}_{0}, \ldots, \boldsymbol{u}_{r}\right\}$ and consider the $K[\boldsymbol{u}]$-module

$$
\mathcal{M}_{\boldsymbol{d}}(V)=K[\boldsymbol{u}]\left[\boldsymbol{x}_{1}, \ldots, \boldsymbol{x}_{m}\right] /\left(I(V)+\left(F_{0}, \ldots, F_{r}\right)\right) .
$$

This module inherits a multigraded structure from $K[\boldsymbol{x}]$. For $\boldsymbol{\delta} \in \mathbb{N}^{m}$, we denote by $\mathcal{M}_{\boldsymbol{d}}(V)_{\boldsymbol{\delta}}$ its part of multidegree $\boldsymbol{\delta}$ in the variables $\boldsymbol{x}$. It is a $K[\boldsymbol{u}]$-module multigraded by setting $\operatorname{deg}\left(u_{i, a}\right)=\boldsymbol{e}_{i} \in \mathbb{N}^{r+1}$, the $(i+1)$-th vector of the standard basis of $\mathbb{R}^{r+1}$.

For the sequel, we fix a set of representatives of the irreducible elements of $K[\boldsymbol{u}]$ made out of primitive polynomials in $A[\boldsymbol{u}]$ and we denote it by $\operatorname{irr}(K[\boldsymbol{u}])$. We recall that the annihilator of a $K[\boldsymbol{u}]$-module $M$ is the ideal of $K[\boldsymbol{u}]$ defined as

$$
\operatorname{Ann}(M)=\operatorname{Ann}_{K[\boldsymbol{u}]}(M)=\{f \in K[\boldsymbol{u}]: f M=0\} .
$$

Definition 1.22. - Let $M$ be a finitely generated $K[\boldsymbol{u}]$-module. If $\operatorname{Ann}(M) \neq 0$, we set

$$
\chi(M)=\chi_{K[\boldsymbol{u}]}(M)=\prod_{f \in \operatorname{irr}(K[\boldsymbol{u}])} f^{\ell\left(M_{(f)}\right)}
$$

where $\ell\left(M_{(f)}\right)$ denotes the length of the $K[\boldsymbol{u}]_{(f)}$-module $M_{(f)}$. In case $\operatorname{Ann}(M)=0$, we set $\chi(M)=0$.

We have that $\ell\left(M_{(f)}\right) \geq 1$ if and only if $\operatorname{Ann}(M) \subset(f)$, see [32, §3.1]. Hence, the product in (1.23) involves a finite number of factors and $\chi(M)$ is well-defined.

Lemma 1.24. - Let $V \subset \mathbb{P}_{K}^{n}$ be an irreducible $K$-variety of dimension $r$ and $\boldsymbol{d} \in\left(\mathbb{N}^{m} \backslash\{\mathbf{0}\}\right)^{r+1}$. Then there exists $\boldsymbol{\delta}_{0} \in \mathbb{N}^{m}$ such that

$$
\operatorname{Ann}\left(\mathcal{M}_{\boldsymbol{d}}(V)_{\boldsymbol{\delta}}\right)=\operatorname{Ann}\left(\mathcal{M}_{\boldsymbol{d}}(V)_{\boldsymbol{\delta}_{0}}\right), \quad \chi\left(\mathcal{M}_{\boldsymbol{d}}(V)_{\boldsymbol{\delta}}\right)=\chi\left(\mathcal{M}_{\boldsymbol{d}}(V)_{\boldsymbol{\delta}_{0}}\right)
$$

for all $\boldsymbol{\delta} \in \mathbb{N}^{m}$ such that $\boldsymbol{\delta} \geq \boldsymbol{\delta}_{0}$.

Proof. - Let $\boldsymbol{\delta}_{\max } \in \mathbb{N}^{m}$ be the maximum of the multidegrees of a set of generators of $\mathcal{M}_{\boldsymbol{d}}(V)$ over $K[\boldsymbol{u}]$. For $\boldsymbol{\delta}^{\prime} \geq \boldsymbol{\delta} \geq \boldsymbol{\delta}_{\max }$ we have that $\mathcal{M}_{\boldsymbol{d}}(V)_{\boldsymbol{\delta}^{\prime}}=K[\boldsymbol{u}][\boldsymbol{x}]_{\boldsymbol{\delta}^{\prime}-\boldsymbol{\delta}} \mathcal{M}_{\boldsymbol{d}}(V)_{\boldsymbol{\delta}}$ and so $\operatorname{Ann}\left(\mathcal{M}_{\boldsymbol{d}}(V)_{\boldsymbol{\delta}^{\prime}}\right) \supset \operatorname{Ann}\left(\mathcal{M}_{\boldsymbol{d}}(V)_{\boldsymbol{\delta}}\right)$. Hence the annihilators of the parts of multidegree $\geq \boldsymbol{\delta}_{\text {max }}$ form an ascending chain of ideals with respect to the order $\leq$ on $\mathbb{N}^{m}$. Eventually, this chain stabilizes because $K[\boldsymbol{u}]$ is Noetherian, which proves the first statement. The second statement is [32, Lem. 3.2]. 
We define eliminants and resultants following [32, Def. 2.14 and §3.2]. The principal part $\operatorname{ppr}(\mathfrak{I})$ of an ideal $\mathfrak{I} \subset K[\boldsymbol{u}]$ is defined as any primitive polynomial in $A[\boldsymbol{u}]$ which is a greatest common divisor of the elements in $\mathfrak{I} \cap A[\boldsymbol{u}]$. If $\mathfrak{I}$ is principal, this polynomial can be equivalently defined as any primitive polynomial in $A[\boldsymbol{u}]$ which is a generator of $\mathfrak{I}$. The principal part of an ideal is unique up to a unit of $A$ and we fix its choice by supposing that it is a product of elements of $\operatorname{irr}(K[\boldsymbol{u}])$.

Definition 1.25. - Let $V \subset \mathbb{P}_{K}^{n}$ be an irreducible $K$-variety of dimension $r \geq 0$ and $\boldsymbol{d} \in\left(\mathbb{N}^{m} \backslash\{\mathbf{0}\}\right)^{r+1}$. The eliminant ideal of $V$ of index $\boldsymbol{d}$ is defined as

$$
\mathfrak{E}_{\boldsymbol{d}}(V)=\operatorname{Ann}\left(\mathcal{M}_{\boldsymbol{d}}(V)_{\boldsymbol{\delta}}\right)
$$

for any $\boldsymbol{\delta} \gg \mathbf{0}$. The eliminant of $V$ of index $\boldsymbol{d}$ is defined as

$$
\operatorname{Elim}_{\boldsymbol{d}}(V)=\operatorname{ppr}\left(\mathfrak{E}_{\boldsymbol{d}}(V)\right)
$$

The eliminant ideal is a non-zero multihomogeneous prime ideal in $K[\boldsymbol{u}]$ [32, Lem. 2.4(2) and Thm. 2.13(1)], see also Proposition 1.37(1) below. In particular, the eliminant is a primitive irreducible multihomogeneous polynomial in $A[\boldsymbol{u}] \backslash\{0\}$.

Definition 1.26. - Let $V \subset \mathbb{P}_{K}^{n}$ be an irreducible $K$-variety of dimension $r \geq 0$ and $\boldsymbol{d} \in\left(\mathbb{N}^{m} \backslash\{\mathbf{0}\}\right)^{r+1}$. The resultant of $V$ of index $\boldsymbol{d}$ is defined as

$$
\operatorname{Res}_{\boldsymbol{d}}(V)=\chi\left(\mathcal{M}_{\boldsymbol{d}}(V)_{\boldsymbol{\delta}}\right)
$$

for any $\boldsymbol{\delta} \gg \mathbf{0}$. It is a non-zero primitive multihomogeneous polynomial in $A[\boldsymbol{u}]$, because of Definition 1.22 and the fact that $\mathfrak{E}_{\boldsymbol{d}}(V)$ is non-zero.

Let $X \in Z_{r}\left(\mathbb{P}_{K}^{\boldsymbol{n}}\right)$ and write $X=\sum_{V} m_{V} V$. The resultant of $X$ of index $\boldsymbol{d}$ is defined as

$$
\operatorname{Res}_{\boldsymbol{d}}(X)=\prod_{V} \operatorname{Res}_{\boldsymbol{d}}(V)^{m_{V}} \in K(\boldsymbol{u})^{\times} .
$$

When $X$ is effective, $\operatorname{Res}_{\boldsymbol{d}}(X)$ is a primitive multihomogeneous polynomial in $A[\boldsymbol{u}]$.

Eliminants and resultants are invariant under index permutations. Next result follows easily from the definitions:

Proposition 1.27. - Let $X \in Z_{r}\left(\mathbb{P}_{K}^{\boldsymbol{n}}\right)$ and $V \subset \mathbb{P}_{K}^{\boldsymbol{n}}$ an irreducible $K$-variety of dimension $r$. Let $\boldsymbol{d}=\left(\boldsymbol{d}_{0}, \ldots, \boldsymbol{d}_{r}\right) \in\left(\mathbb{N}^{m} \backslash\{\mathbf{0}\}\right)^{r+1}$ and $\boldsymbol{u}=\left(\boldsymbol{u}_{0}, \ldots, \boldsymbol{u}_{r}\right)$ the group of variables corresponding to $\boldsymbol{d}$. Let $\sigma$ be a permutation of the set $\{0, \ldots, r\}$ and write $\sigma \boldsymbol{d}=\left(\boldsymbol{d}_{\sigma(0)}, \ldots, \boldsymbol{d}_{\sigma(r)}\right), \sigma \boldsymbol{u}=\left(\boldsymbol{u}_{\sigma(0)}, \ldots, \boldsymbol{u}_{\sigma(r)}\right)$. Then $\operatorname{Res}_{\sigma \boldsymbol{d}}(X)(\sigma \boldsymbol{u})=\operatorname{Res}_{\boldsymbol{d}}(X)(\boldsymbol{u})$ and $\operatorname{Elim}_{\sigma \boldsymbol{d}}(V)(\sigma \boldsymbol{u})=\operatorname{Elim}_{\boldsymbol{d}}(V)(\boldsymbol{u})$.

Eliminants and resultants are also invariant under field extensions.

Proposition 1.28. - Let $X \in Z_{r}\left(\mathbb{P}_{K}^{n}\right), V \subset \mathbb{P}_{K}^{n}$ an irreducible $K$-variety of dimension $r$ and $\boldsymbol{d}=\left(\boldsymbol{d}_{0}, \ldots, \boldsymbol{d}_{r}\right) \in\left(\mathbb{N}^{m} \backslash\{\mathbf{0}\}\right)^{r+1}$. Let $K \subset E$ be a field extension. Then there exists $\lambda_{1} \in E^{\times}$such that $\operatorname{Res}_{\boldsymbol{d}}\left(X_{E}\right)=\lambda_{1} \operatorname{Res}_{\boldsymbol{d}}(X)$. Furthermore, if $V_{E}$ is an irreducible E-variety, then there exists $\lambda_{2} \in E^{\times}$such that $\operatorname{Elim}_{\boldsymbol{d}}\left(V_{E}\right)=\lambda_{2} \operatorname{Elim}_{\boldsymbol{d}}(V)$.

To prove this, we need the following lemma. 
Lemma 1.29. - Let $M$ be a finitely generated $K[\boldsymbol{u}]$-module and $K \subset E$ a field extension. Then $\operatorname{Ann}_{E[\boldsymbol{u}]}\left(M \otimes_{K} E\right)=\operatorname{Ann}_{K[\boldsymbol{u}]}(M) \otimes_{K} E$ and $\chi_{E}\left(M \otimes_{K} E\right)=\lambda \chi_{K}(M)$ with $\lambda \in E^{\times}$.

Proof. - The first statement is a consequence of the fact that $E$ is a flat $K$-module: for each $m \in M$, the exact sequence

$$
0 \rightarrow \operatorname{Ann}_{K[\boldsymbol{u}]}(m) \rightarrow K[\boldsymbol{u}] \rightarrow K[\boldsymbol{u}] m \rightarrow 0
$$

yields the tensored exact sequence

$$
0 \rightarrow \operatorname{Ann}_{K[\boldsymbol{u}]}(m) \otimes_{K} E \rightarrow E[\boldsymbol{u}] \rightarrow E[\boldsymbol{u}]\left(m \otimes_{K} 1\right) \rightarrow 0 .
$$

Hence, $\operatorname{Ann}_{E[\boldsymbol{u}]}\left(m \otimes_{K} 1\right)=\operatorname{Ann}_{K[\boldsymbol{u}]}(m) \otimes_{K} E$. Now, if $M=\left(m_{1}, \ldots, m_{\ell}\right)$, then

$$
\begin{aligned}
\operatorname{Ann}_{E[\boldsymbol{u}]}\left(M \otimes_{K} E\right)=\bigcap_{i} \operatorname{Ann}_{E[\boldsymbol{u}]}\left(m_{i}\right. & \left.\otimes_{K} 1\right) \\
& =\bigcap_{i} \operatorname{Ann}_{K[\boldsymbol{u}]}\left(m_{i}\right) \otimes_{K} E=\operatorname{Ann}_{K[\boldsymbol{u}]}(M) \otimes_{K} E .
\end{aligned}
$$

For the second statement, we can reduce to the case when $\operatorname{Ann}(M) \neq 0$ because otherwise it is trivial. Let $f \in \operatorname{irr}(K[\boldsymbol{u}])$. The localization $K[\boldsymbol{u}]_{(f)}$ is a principal local domain and so $M_{(f)} \simeq \bigoplus_{i=1}^{N} K[\boldsymbol{u}]_{(f)} /\left(f^{\nu_{i}}\right)$ for some $\nu_{i} \geq 1$. In particular, $\ell\left(M_{(f)}\right)=\sum_{i} \nu_{i}$. Let $f=\lambda_{f} \prod_{g} g^{\mu_{g}}$ be the factorization of $f$ into elements $g \in \operatorname{irr}(E[\boldsymbol{u}])$ and a non-zero constant $\lambda_{f} \in E^{\times}$. On the one hand, for each $g$ in this factorization,

$$
\left(M \otimes_{K} E\right)_{(g)} \simeq M_{(f)} \otimes_{K[\boldsymbol{u}]} E[\boldsymbol{u}]_{(g)} \simeq \bigoplus_{i=1}^{N} E[\boldsymbol{u}]_{(g)} /\left(g^{\mu_{g} \nu_{i}}\right) .
$$

Hence $\ell\left(\left(M \otimes_{K} E\right)_{(g)}\right)=\left(\sum \nu_{i}\right) \mu_{g}=\ell\left(M_{(f)}\right) \mu_{g}$. On the other hand, let $g \in \operatorname{irr}(E[\boldsymbol{u}])$ be an irreducible polynomial which does not divide any $f \in \operatorname{irr}(K[\boldsymbol{u}])$ and suppose that $\ell\left(\left(M \otimes_{K} E\right)_{(g)}\right) \geq 1$. If this were the case, we would have $\operatorname{Ann}_{E[\boldsymbol{u}]}\left(M \otimes_{K} E\right) \subset(g)$. By the (already proved) first part of this proposition,

$$
\operatorname{Ann}_{E[\boldsymbol{u}]}\left(M \otimes_{K} E\right)=\operatorname{Ann}_{K[\boldsymbol{u}]}(M) \otimes_{K} E .
$$

This implies $\operatorname{Ann}(M) \subset(g) \cap K[\boldsymbol{u}]=0$, which contradicts the assumption that $\operatorname{Ann}(M) \neq 0$. Therefore, $\ell\left(\left(M \otimes_{K} E\right)_{(g)}\right)=0$. We deduce

$$
\prod_{g \in \operatorname{irr}(E[\boldsymbol{u}])} g^{\ell\left((M \otimes E)_{(g)}\right)}=\prod_{f \in \operatorname{irr}(K[\boldsymbol{u}])}\left(\prod_{g \mid f} g^{\ell\left(M_{(f)}\right) \mu_{g}}\right)=\lambda \prod_{f} f^{\ell\left(M_{(f)}\right)}
$$

for $\lambda=\prod_{f} \lambda_{f}^{-\ell\left(M_{(f)}\right)} \in E^{\times}$. Hence $\chi_{E[u]}\left(M \otimes_{K} E\right)=\lambda \chi_{K[u]}(M)$, as stated.

Proof of Proposition 1.28. - To prove the first part, it is enough to consider the case of an irreducible $K$-variety $V$. By definition, $V_{E}$ is the effective $E$-cycle defined by the extended ideal $I(V) \otimes_{K} E$. Hence,

$$
\mathcal{M}_{\boldsymbol{d}}\left(V_{E}\right)=E[\boldsymbol{u}]\left[\boldsymbol{x}_{1}, \ldots, \boldsymbol{x}_{m}\right] /\left(I(V) \otimes_{K} E+\left(F_{0}, \ldots, F_{r}\right)\right)=\mathcal{M}_{\boldsymbol{d}}(V) \otimes_{K} E .
$$

By [32, Thm. 3.3] and Lemma 1.29,

$$
\operatorname{Res}_{\boldsymbol{d}}\left(V_{E}\right)=\chi_{E[u]}\left(\mathcal{M}_{\boldsymbol{d}}\left(V_{E}\right)_{\boldsymbol{\delta}}\right)=\lambda \chi_{K[u]}\left(\mathcal{M}_{\boldsymbol{d}}(V)_{\boldsymbol{\delta}}\right)=\lambda_{1} \operatorname{Res}_{\boldsymbol{d}}(V)
$$


for any $\delta \gg 0$ and a $\lambda_{1} \in E^{\times}$, which proves the first part of the statement. The second part follows similarly from the definition of eliminants and Lemma 1.29.

Proposition 1.30. - Let $V \subset \mathbb{P}_{K}^{n}$ be an irreducible $K$-variety of dimension $r$ and $\boldsymbol{d} \in\left(\mathbb{N}^{m} \backslash\{\mathbf{0}\}\right)^{r+1}$. Then there exists $\nu \geq 1$ such that

$$
\operatorname{Res}_{\boldsymbol{d}}(V)=\operatorname{Elim}_{\boldsymbol{d}}(V)^{\nu} .
$$

Proof. - Let $\boldsymbol{\delta} \in \mathbb{N}^{m}$ and $f \in \operatorname{irr}(K[\boldsymbol{u}])$. We have that $\left(\mathcal{M}_{\boldsymbol{d}}(V)_{\boldsymbol{\delta}}\right)_{(f)} \neq 0$ if and only if $\operatorname{Ann}\left(\mathcal{M}_{\boldsymbol{d}}(V)_{\boldsymbol{\delta}}\right) \subset(f)$. Therefore,

$$
f\left|\operatorname{Res}_{\boldsymbol{d}}(V) \Longleftrightarrow f\right| \operatorname{Elim}_{\boldsymbol{d}}(V) .
$$

Thus $\operatorname{Elim}_{\boldsymbol{d}}(V)$ is the only irreducible factor of $\operatorname{Res}_{\boldsymbol{d}}(V)$ and the statement follows.

EXAMPLE 1.31. - The resultant of an irreducible variety is not necessarily an irreducible polynomial: consider the curve $C=V\left(x_{1,0}^{2} x_{2,1}-x_{1,1}^{2} x_{2,0}\right) \subset \mathbb{P}^{1} \times \mathbb{P}^{1}$ and the indexes $\boldsymbol{d}_{0}=\boldsymbol{d}_{1}=(0,1)$ with associated linear forms $F_{i}=u_{i, 0} x_{2,0}+u_{i, 1} x_{2,1}$ for $i=0,1$. We can verify that the corresponding resultant is

$$
\operatorname{Res}_{\boldsymbol{d}_{0}, \boldsymbol{d}_{1}}(C)=\left(u_{0,0} u_{1,1}-u_{0,1} u_{1,0}\right)^{2} .
$$

The partial degrees of resultants can be expressed in terms of mixed degrees.

Proposition 1.32. - Let $X \in Z_{r}^{+}\left(\mathbb{P}_{K}^{\boldsymbol{n}}\right)$ and $\boldsymbol{d} \in\left(\mathbb{N}^{m} \backslash\{\mathbf{0}\}\right)^{r+1}$. Then, for $0 \leq i \leq r$,

$$
\operatorname{deg}_{\boldsymbol{u}_{i}}\left(\operatorname{Res}_{\boldsymbol{d}}(X)\right)=\operatorname{coeff}_{\boldsymbol{\theta}^{n}}\left([X] \prod_{j \neq i}\left(\sum_{\ell=1}^{m} d_{j, \ell} \theta_{\ell}\right)\right) .
$$

Proof. - This follows from [32, Prop. 3.4].

For projective varieties, eliminants and resultants coincide:

Corollary 1.33. - For $n \in \mathbb{N}$ let $V \subset \mathbb{P}_{K}^{n}$ be an irreducible $K$-variety of dimension $r$ and $\boldsymbol{d} \in\left(\mathbb{Z}_{>0}\right)^{r+1}$. Then $\operatorname{Res}_{\boldsymbol{d}}(V)=\operatorname{Elim}_{\boldsymbol{d}}(V)$.

Proof. - By Proposition 1.30, $\operatorname{Res}_{\boldsymbol{d}}(V)=\operatorname{Elim}_{\boldsymbol{d}}(V)^{\nu}$ for some $\nu \geq 1$. On the one hand, $\operatorname{deg}_{\boldsymbol{u}_{i}}\left(\operatorname{Elim}_{\boldsymbol{d}}(V)\right)=\left(\prod_{j \neq i} d_{j}\right) \operatorname{deg}(V)$ for all $i$ [27, Remark to Lem. 1.8] while on the other hand, Proposition 1.32 implies that

$$
\operatorname{deg}_{\boldsymbol{u}_{i}}\left(\operatorname{Res}_{\boldsymbol{d}}(V)\right)=\operatorname{coeff}_{\theta^{n}}\left(\operatorname{deg}(V) \theta^{n-r} \prod_{j \neq i} d_{j} \theta\right)=\left(\prod_{j \neq i} d_{j}\right) \operatorname{deg}(V) .
$$

Thus $\operatorname{Res}_{\boldsymbol{d}}(V)$ and $\operatorname{Elim}_{\boldsymbol{d}}(V)$ have the same total degree. Hence, $\nu=1$ and the statement follows.

Given a subset $J \subset\{1, \ldots, m\}$ we set $\pi_{J}: \mathbb{P}_{K}^{n} \rightarrow \prod_{j \in J} \mathbb{P}_{K}^{n_{j}}$ for the natural projection and $\boldsymbol{x}_{J}=\left(\boldsymbol{x}_{j}\right)_{j \in J}$.

$4^{\text {e }}$ SÉRIE - TOME $46-2013-$ No $^{\circ}$ 
Lemma 1.34. - Let $\boldsymbol{d} \in\left(\mathbb{N}^{m} \backslash\{\mathbf{0}\}\right)^{r+1}$ and $F_{i}$ the associated general form of multidegree $\boldsymbol{d}_{i}$ for $0 \leq i \leq r$. Let $V \subset \mathbb{P}_{K}^{n}$ be an irreducible $K$-variety of dimension $r \geq 0$. Then $\mathfrak{E}_{\boldsymbol{d}}(V)$ is a principal ideal if and only if

$$
\operatorname{dim}\left(\pi_{J}(V)\right) \geq \#\left\{i: 0 \leq i \leq r, F_{i} \in K\left[\boldsymbol{u}_{i}\right]\left[\boldsymbol{x}_{J}\right]\right\}-1 \quad \text { for all } J \subset\{1, \ldots, m\} .
$$

If this is the case, $\operatorname{Elim}_{\boldsymbol{d}}(V) \in K[\boldsymbol{u}] \backslash K$. Otherwise, $\mathfrak{E}_{\boldsymbol{d}}(V)$ is not principal and $\operatorname{Elim}_{\boldsymbol{d}}(V)=1$.

Proof. - Assume for the moment that the field $K$ is infinite.

$(\Leftarrow)$ If $(1.35)$ holds, [32, Cor. 2.15(2)] implies that $\operatorname{Elim}_{\boldsymbol{d}}(V)$ generates $\mathfrak{E}_{\boldsymbol{d}}(V)$. Applying [32, Cor. 2.15(1)], it follows $\operatorname{Elim}_{\boldsymbol{d}}(V) \neq 0$ since, for $J=\{1, \ldots, m\}$,

$$
\operatorname{dim}\left(\pi_{J}(V)\right)=\operatorname{dim}(V)=r=\#\left\{i \in\{0, \ldots, r\}: F_{i} \in K\left[\boldsymbol{u}_{i}\right][\boldsymbol{x}]\right\}-1 .
$$

Now suppose that $\operatorname{Elim}_{\boldsymbol{d}}(V)=1$. This is equivalent to the fact that $\mathcal{M}_{\boldsymbol{d}}(V)_{\boldsymbol{\delta}}=0$ for $\boldsymbol{\delta} \gg 0$, which implies $I(V) \supset\left(\mathfrak{M}_{\boldsymbol{n}}\right)_{\boldsymbol{\delta}}$ and so $V=\varnothing$, which is a contradiction. Therefore, $\operatorname{Elim}_{\boldsymbol{d}}(V) \in K[\boldsymbol{u}] \backslash K$.

$(\Rightarrow)$ Suppose that (1.35) does not hold. By [32, Cor. 2.15(3)], $\operatorname{Elim}_{\boldsymbol{d}}(V)=1$. Hence $\mathfrak{E}_{\boldsymbol{d}}(V)$ is necessarily not principal, because otherwise we would have that $V=\varnothing$.

The case when $K$ is a finite field reduces to the previous case, by considering any transcendental extension $E$ of $K$ and applying Proposition 1.28.

Given $\boldsymbol{d} \in\left(\mathbb{N}^{m} \backslash\{\mathbf{0}\}\right)^{r+1}$, the space of coefficients of a family of multihomogeneous polynomials in $\bar{K}[\boldsymbol{x}] \backslash\{0\}$ of multidegrees $\boldsymbol{d}_{0}, \ldots, \boldsymbol{d}_{r}$ can be identified with

$$
\mathbb{P}^{\boldsymbol{N}}:=\prod_{i=0}^{r} \mathbb{P}^{\prod_{j=1}^{m}\left(\begin{array}{c}
d_{i, j}+n_{j} \\
n_{j}
\end{array}\right)-1}
$$

For $V \subset \mathbb{P}^{n}$ consider the following subset of $\mathbb{P}^{N}$ :

$$
\nabla_{\boldsymbol{d}}(V)=\left\{\left(\boldsymbol{u}_{0}, \ldots, \boldsymbol{u}_{r}\right) \in \mathbb{P}^{\boldsymbol{N}}: V \cap V\left(F_{0}\left(\boldsymbol{u}_{0}, \boldsymbol{x}\right), \ldots, F_{r}\left(\boldsymbol{u}_{r}, \boldsymbol{x}\right)\right) \neq \varnothing\right\} .
$$

The following results give a geometric interpretation of eliminant ideals.

Proposition 1.37. - Let $V \subset \mathbb{P}^{n}$ be an irreducible variety of dimension $r \geq 0$ and $\boldsymbol{d} \in\left(\mathbb{N}^{m} \backslash\{\mathbf{0}\}\right)^{r+1}$. Then

1. $I\left(\nabla_{\boldsymbol{d}}(V)\right)=\mathfrak{E}_{\boldsymbol{d}}(V)$;

2. the variety $\nabla_{\boldsymbol{d}}(V)$ is a hypersurface if and only if (1.35) holds. If this is the case, $\nabla_{\boldsymbol{d}}(V)=V\left(\operatorname{Elim}_{\boldsymbol{d}}(V)\right)=V\left(\operatorname{Res}_{\boldsymbol{d}}(V)\right)$.

Proof. - (1) follows from [32, Thm. 2.2], while (2) follows from (1) together with Lemma 1.34 and Proposition 1.30.

The following corollary gives a formula $\grave{a}$ la Poisson for the resultants of a cycle of dimension 0 . Recall that the evaluation of a multihomogeneous polynomial at a point of $\mathbb{P}^{n}$ is only defined up to a non-zero constant in $\bar{K}^{\times}$which depends on a choice of a representative of the given point. 
Corollary 1.38. - Let $X \in Z_{0}\left(\mathbb{P}_{K}^{\boldsymbol{n}}\right)$ and $\boldsymbol{d}_{0} \in \mathbb{N}^{m} \backslash\{\mathbf{0}\}$. Write $X_{\bar{K}}=\sum_{\boldsymbol{\xi}} m_{\boldsymbol{\xi}} \boldsymbol{\xi}$ with $\boldsymbol{\xi} \in \mathbb{P}^{\boldsymbol{n}}$ and $m_{\boldsymbol{\xi}} \in \mathbb{Z}$ and let $F_{0}$ be the general form of multidegree $\boldsymbol{d}_{0}$. Then there exists $\lambda \in \bar{K}^{\times}$ such that

$$
\operatorname{Res}_{\boldsymbol{d}_{0}}(X)=\lambda \prod_{\boldsymbol{\xi}} F_{0}(\boldsymbol{\xi})^{m_{\boldsymbol{\xi}}}
$$

Proof. - Let $\boldsymbol{\xi} \in \mathbb{P}^{\boldsymbol{n}}$. Observe that $F_{0}(\boldsymbol{\xi})=0$ is the irreducible equation of the hypersurface $\nabla_{\boldsymbol{d}}(\boldsymbol{\xi})$. By Proposition 1.37, there exists $\lambda \in \bar{K}^{\times}$such that $\operatorname{Elim}_{\boldsymbol{d}_{0}}(\boldsymbol{\xi})=\lambda F_{0}(\boldsymbol{\xi})$. Propositions 1.32 and 1.10(3) imply that $\operatorname{deg}_{\boldsymbol{u}_{0}}\left(\operatorname{Res}_{\boldsymbol{d}}(\boldsymbol{\xi})\right)=1$. Applying Proposition 1.30, we get $\operatorname{Res}_{\boldsymbol{d}_{0}}(\boldsymbol{\xi})=\operatorname{Elim}_{\boldsymbol{d}_{0}}(\boldsymbol{\xi})$. The general case follows readily from the definition of the resultant and its invariance under field extensions.

REMARK 1.39. - The notions of eliminant and resultant of multiprojective cycles include several of the classical notions of resultant.

1. The Macaulay resultant [23]. The classical resultant of $n+1$ homogeneous polynomials of degrees $d_{0}, \ldots, d_{n}$ coincides both with $\operatorname{Elim}_{\left(d_{0}, \ldots, d_{n}\right)}\left(\mathbb{P}^{n}\right)$ and with $\operatorname{Res}_{\left(d_{0}, \ldots, d_{n}\right)}\left(\mathbb{P}^{n}\right)$. This is a consequence of Proposition 1.37(2) and Corollary 1.33.

2. Chow forms [8]. The Chow form of an irreducible variety $V \subset \mathbb{P}^{n}$ of dimension $r$ coincides both with $\operatorname{Elim}_{(1, \ldots, 1)}(V)$ and with $\operatorname{Res}_{(1, \ldots, 1)}(V)$. This follows from Proposition 1.37 and Corollary 1.33 .

3. The GKZ mixed resultant $[12, \S 3.3]$. Let $V$ be a proper irreducible variety over $\mathbb{C}$ of dimension $r$ equipped with a family of very ample line bundles $L_{0}, \ldots, L_{r}$ and $R_{L_{0}, \ldots, L_{r}}$ the $\left(L_{0}, \ldots, L_{r}\right)$-resultant of $V$ in the sense of I. Gel'fand, M. Kapranov and A. Zelevinski. Each $L_{i}$ defines an embedding $\psi_{i}: V \hookrightarrow \mathbb{P}^{n_{i}}$. We consider then the map

$$
\boldsymbol{\psi}: V \longleftrightarrow \mathbb{P}^{\boldsymbol{n}}, \quad \boldsymbol{\xi} \longmapsto\left(\psi_{0}(\boldsymbol{\xi}), \ldots, \psi_{r}(\boldsymbol{\xi})\right) .
$$

Using Proposition 1.37(2), it can be shown that $R_{L_{0}, \ldots, L_{r}}$ coincides with the eliminant form $\operatorname{Elim}_{\boldsymbol{e}_{0}, \ldots, \boldsymbol{e}_{r}}(\boldsymbol{\psi}(V))$. Using the formula for the degree of the GKZ mixed resultant in [12, Thm. 3.3], we can show that it also coincides with the resultant $\operatorname{Res}_{\boldsymbol{e}_{0}, \ldots, \boldsymbol{e}_{r}}(\boldsymbol{\psi}(V))$.

\subsection{Operations on resultants}

We will now study the behavior of resultants with respect to basic geometric operations, including intersections, linear projections and products of cycles.

An important feature of resultants is that they transform the intersection product of a cycle with a divisor into an evaluation.

Proposition 1.40. - Let $X \in Z^{r}\left(\mathbb{P}_{K}^{\boldsymbol{n}}\right)$ and $\boldsymbol{d}_{0}, \ldots, \boldsymbol{d}_{r} \in \mathbb{N}^{m} \backslash\{\mathbf{0}\}$. Let $f \in K[\boldsymbol{x}]_{\boldsymbol{d}_{r}}$ such that $\operatorname{div}(f)$ intersects $X$ properly. Then there exists $\lambda \in K^{\times}$such that

$$
\operatorname{Res}_{\boldsymbol{d}_{0}, \ldots, \boldsymbol{d}_{r-1}, \boldsymbol{d}_{r}}(X)\left(\boldsymbol{u}_{0}, \ldots, \boldsymbol{u}_{r-1}, f\right)=\lambda \operatorname{Res}_{\boldsymbol{d}_{0}, \ldots, \boldsymbol{d}_{r-1}}(X \cdot \operatorname{div}(f))\left(\boldsymbol{u}_{0}, \ldots, \boldsymbol{u}_{r-1}\right),
$$

where the left-hand side denotes the specialization of the last group of variables of $\operatorname{Res}_{\boldsymbol{d}_{0}, \ldots, \boldsymbol{d}_{r-1}, \boldsymbol{d}_{r}}(X)$ at the coefficients of $f$.

Proof. - This is [32, Prop. 3.6].

4 ${ }^{\text {e }}$ SÉRIE - TOME 46 - 2013 - No 4 
Next we consider the behavior of resultants with respect to standard projections. Consider the linear projection $\pi: \mathbb{P}_{K}^{\boldsymbol{n}} \rightarrow \mathbb{P}_{K}^{\boldsymbol{l}}$ in (1.15) and let $\boldsymbol{x}$ and $\boldsymbol{y}$ denote the multihomogeneous coordinates of $\mathbb{P}_{K}^{n}$ and of $\mathbb{P}_{K}^{\boldsymbol{l}}$, respectively. Let $\boldsymbol{d} \in\left(\mathbb{N}^{m} \backslash\{\mathbf{0}\}\right)^{r+1}$. The general forms of multidegree $\boldsymbol{d}_{i}$ in the variables $\boldsymbol{x}$ and $\boldsymbol{y}$ are, respectively,

$$
F_{i}=\sum_{\boldsymbol{a} \in \mathbb{N}_{d_{i}}^{n+1}} u_{i, \boldsymbol{a}} \boldsymbol{x}^{\boldsymbol{a}} \in K\left[\boldsymbol{u}_{i}\right][\boldsymbol{x}], \quad F_{i}^{\prime}=\sum_{\boldsymbol{a} \in \mathbb{N}_{\boldsymbol{d}_{i}}^{l+1}} u_{i, \boldsymbol{a}} \boldsymbol{y}^{\boldsymbol{a}} \in K\left[\boldsymbol{u}_{i}\right][\boldsymbol{y}]
$$

Write

$$
\boldsymbol{u}_{i}^{\prime}=\left\{u_{i, \boldsymbol{a}}: \boldsymbol{a} \in \mathbb{N}_{\boldsymbol{d}_{i}}^{l+\mathbf{1}}\right\}, \quad \boldsymbol{u}_{i}^{\prime \prime}=\left\{u_{i, \boldsymbol{a}}: \boldsymbol{a} \in \mathbb{N}_{\boldsymbol{d}_{i}}^{\boldsymbol{n}+\mathbf{1}} \backslash \mathbb{N}_{\boldsymbol{d}_{i}}^{\boldsymbol{l + 1}}\right\}
$$

Let $\prec$ be the partial monomial order on $K\left[\boldsymbol{u}_{0}, \ldots, \boldsymbol{u}_{r}\right]$ defined as

$$
\left\{\boldsymbol{u}_{i}^{\prime}\right\}_{0 \leq i \leq r} \prec \boldsymbol{u}_{0}^{\prime \prime} \prec \cdots \prec \boldsymbol{u}_{r}^{\prime \prime} .
$$

By this, we mean that the variables in each set have the same weight and that those in $\boldsymbol{u}_{r}^{\prime \prime}$ have the maximal weight, then come those in $\boldsymbol{u}_{r-1}^{\prime \prime}$, etcetera. Observe that this order can be alternatively defined as the lexicographic order associated to the sequence of vectors $\boldsymbol{w}_{0}, \ldots, \boldsymbol{w}_{r}$ defined as

$$
\boldsymbol{w}_{i}=(\overbrace{(\mathbf{0}, \mathbf{0}), \ldots,(\mathbf{0}, \mathbf{0})}^{i-1},(\mathbf{0}, \mathbf{1}), \overbrace{(\mathbf{0}, \mathbf{0}), \ldots,(\mathbf{0}, \mathbf{0})}^{r-i}) .
$$

Given a polynomial $F \in K[\boldsymbol{u}] \backslash\{0\}$, we denote by $\operatorname{init}_{\prec}(F) \in K[\boldsymbol{u}] \backslash\{0\}$ its initial part with respect to this order. It consists in the sum of the terms in $F$ whose monomials are minimal with respect to $\prec$. This order is multiplicative, in the sense that $\operatorname{init}_{\prec}(F G)=$ $\operatorname{init}_{\prec}(F) \operatorname{init}_{\prec}(G)$ for all $F, G \in K[\boldsymbol{u}]$.

Proposition 1.41. - Let $\pi: \mathbb{P}_{K}^{n} \rightarrow \mathbb{P}_{K}^{l}$ be the linear projection and $\prec$ the partial monomial order on $K[\boldsymbol{u}]$ considered above. Let $X \in Z_{r}^{+}\left(\mathbb{P}_{K}^{\boldsymbol{n}}\right)$ and $\boldsymbol{d} \in\left(\mathbb{N}^{m} \backslash\{\mathbf{0}\}\right)^{r+1}$. Then

$$
\operatorname{Res}_{\boldsymbol{d}}\left(\pi_{*} X\right) \mid \operatorname{init}_{\prec}\left(\operatorname{Res}_{\boldsymbol{d}}(X)\right) \quad \text { in } A[\boldsymbol{u}] \text {. }
$$

Proof. - It is enough to consider the case $\pi_{*}(X) \neq 0$, otherwise $\operatorname{Res}_{\boldsymbol{d}}\left(\pi_{*} X\right)=1$. In addition, we only need to prove that the division holds in $K[\boldsymbol{u}]$, since both polynomials belong to $A[\boldsymbol{u}]$ and $\operatorname{Res}_{\boldsymbol{d}}\left(\pi_{*} X\right)$ is primitive.

We proceed by induction on the dimension $r$. For $r=0$, write $X_{\bar{K}}=\sum_{\boldsymbol{\xi}} m_{\boldsymbol{\xi}} \boldsymbol{\xi}$ with $\boldsymbol{\xi} \in \mathbb{P}^{n}$ and $m_{\boldsymbol{\xi}} \in \mathbb{Z}$. Hence,

$$
\pi_{*} X_{\bar{K}}=\sum_{\boldsymbol{\xi} \notin L} m_{\boldsymbol{\xi}} \pi_{*} \boldsymbol{\xi}
$$

since $\pi_{*} \boldsymbol{\xi}=0$ whenever $\boldsymbol{\xi} \in L$, with $L$ defined in (1.15). Observe that $F_{0}^{\prime}(\pi(\boldsymbol{\xi}))=$ init $_{\prec}\left(F_{0}(\boldsymbol{\xi})\right)$ for each $\boldsymbol{\xi} \notin L$. Using Corollary 1.38 and the multiplicativity of the order $\prec$, we deduce that there exist $\lambda, \lambda^{\prime} \in \bar{K}^{\times}$such that

$$
\operatorname{Res}_{\boldsymbol{d}_{0}}\left(\pi_{*} X\right)=\lambda^{\prime} \prod_{\boldsymbol{\xi} \notin L} \operatorname{init}_{\prec}\left(F_{0}(\boldsymbol{\xi})\right)^{m_{\boldsymbol{\xi}}} \mid \operatorname{init}_{\prec}\left(\prod_{\boldsymbol{\xi}} F_{0}(\boldsymbol{\xi})^{m_{\boldsymbol{\xi}}}\right)=\lambda \operatorname{init}_{\prec}\left(\operatorname{Res}_{\boldsymbol{d}_{0}}(\boldsymbol{\xi})\right) .
$$

Now let $r \geq 1$ and suppose that we have proved the statement for all cycles of pure dimension up to $r-1$ and any base field. Let $V$ be an irreducible $K$-variety of dimension $r$. We suppose that $\operatorname{Res}_{\boldsymbol{d}}\left(\pi_{*} V\right) \neq 1$ because otherwise the statement is trivial. Thus, the degree of this resultant in some group of variables is $\geq 1$ and, up to a reordering, we can suppose that 
this holds for the group $\boldsymbol{u}_{0}^{\prime}$. Consider the scalar extension $V_{K\left(\boldsymbol{u}_{r}^{\prime}\right)}$ of the $K$-variety $V$ by the field $K\left(\boldsymbol{u}_{r}^{\prime}\right)$. By Proposition 1.28, there exists $\lambda_{1} \in K\left(\boldsymbol{u}_{r}^{\prime}\right)^{\times}$such that

$$
\operatorname{Res}_{\boldsymbol{d}}\left(\pi_{*} V\right)=\lambda_{1} \operatorname{Res}_{\boldsymbol{d}}\left(\pi_{*} V_{K\left(\boldsymbol{u}_{r}^{\prime}\right)}\right) .
$$

In turn, Proposition 1.40 implies that there exists $\lambda_{2} \in K\left(\boldsymbol{u}_{r}^{\prime}\right)^{\times}$such that

$$
\operatorname{Res}_{\boldsymbol{d}}\left(\pi_{*}\left(V_{K\left(\boldsymbol{u}_{r}^{\prime}\right)}\right)\right)=\lambda_{2} \operatorname{Res}_{\boldsymbol{d}_{0}, \ldots, \boldsymbol{d}_{r-1}}\left(\pi_{*}\left(V_{K\left(\boldsymbol{u}_{r}^{\prime}\right)}\right) \cdot \operatorname{div}_{\mathbb{P}^{l}}\left(F_{r}^{\prime}\right)\right)\left(\boldsymbol{u}_{0}, \ldots, \boldsymbol{u}_{r-1}\right) .
$$

We can verify that the form $F_{r}^{\prime}$ satisfies the hypothesis of Lemma 1.18. Hence, this lemma implies the equality of cycles $\pi_{*}\left(V_{K\left(\boldsymbol{u}_{r}^{\prime}\right)} \cdot \operatorname{div}_{\mathbb{P}^{n}}\left(F_{r}^{\prime}\right)\right)=\pi_{*}\left(V_{K\left(\boldsymbol{u}_{r}^{\prime}\right)}\right) \cdot \operatorname{div}_{\mathbb{P}^{l}}\left(F_{r}^{\prime}\right)$. In particular,

$$
\operatorname{Res}_{\boldsymbol{d}_{0}, \ldots, \boldsymbol{d}_{r-1}}\left(\pi_{*}\left(V_{K\left(\boldsymbol{u}_{r}^{\prime}\right)}\right) \cdot \operatorname{div}_{\mathbb{P} l}\left(F_{r}^{\prime}\right)\right)=\operatorname{Res}_{\boldsymbol{d}_{0}, \ldots, \boldsymbol{d}_{r-1}}\left(\pi_{*}\left(V_{K\left(\boldsymbol{u}_{r}^{\prime}\right)} \cdot \operatorname{div}_{\mathbb{P} n}\left(F_{r}^{\prime}\right)\right)\right) .
$$

Applying the inductive hypothesis to the $(r-1)$-dimensional cycle $V_{K\left(\boldsymbol{u}_{r}^{\prime}\right)} \cdot \operatorname{div}_{\mathbb{P} n}\left(F_{r}^{\prime}\right)$,

$$
\operatorname{Res}_{\boldsymbol{d}_{0}, \ldots, \boldsymbol{d}_{r-1}}\left(\pi_{*}\left(V_{K\left(\boldsymbol{u}_{r}^{\prime}\right)} \cdot \operatorname{div}_{\mathbb{P} n}\left(F_{r}^{\prime}\right)\right)\right) \mid \operatorname{init}_{\prec}\left(\operatorname{Res}_{\boldsymbol{d}_{0}, \ldots, \boldsymbol{d}_{r-1}}\left(V_{K\left(\boldsymbol{u}_{r}^{\prime}\right)} \cdot \operatorname{div}_{\mathbb{P} \boldsymbol{n}}\left(F_{r}^{\prime}\right)\right)\right) .
$$

The divisor $\operatorname{div}_{\mathbb{P}^{n}}\left(F_{r}^{\prime}\right)$ intersects $V_{K\left(\boldsymbol{u}_{r}^{\prime}\right)}$ properly. Proposition 1.40 then implies

$$
\operatorname{Res}_{\boldsymbol{d}_{0}, \ldots, \boldsymbol{d}_{r-1}}\left(V_{K\left(\boldsymbol{u}_{r}^{\prime}\right)} \cdot \operatorname{div}_{\mathbb{P} n}\left(F_{r}^{\prime}\right)\right)\left(\boldsymbol{u}_{0}, \ldots, \boldsymbol{u}_{r-1}\right)=\lambda_{3} \operatorname{Res}_{\boldsymbol{d}}(V)\left(\boldsymbol{u}_{0}, \ldots, \boldsymbol{u}_{r-1}, F_{r}^{\prime}\right)
$$

for some $\lambda_{3} \in K\left(\boldsymbol{u}_{r}^{\prime}\right)^{\times}$. This last polynomial is not zero and it satisfies

$$
\operatorname{init}_{\prec}\left(\operatorname{Res}_{\boldsymbol{d}}(V)\left(\boldsymbol{u}_{0}, \ldots, \boldsymbol{u}_{r-1}, F_{r}^{\prime}\right)\right)=\operatorname{init}_{\prec}\left(\operatorname{Res}_{\boldsymbol{d}}(V)\right),
$$

due to the definition of $\prec$. We conclude

$$
\operatorname{Res}_{\boldsymbol{d}}\left(\pi_{*} V\right) \mid \operatorname{init}_{\prec}\left(\operatorname{Res}_{\boldsymbol{d}}(V)\right) \quad \text { in } K\left(\boldsymbol{u}_{r}^{\prime}\right)\left[\boldsymbol{u}_{0}, \ldots, \boldsymbol{u}_{r-1}\right] .
$$

This readily implies that $\operatorname{Res}_{\boldsymbol{d}}\left(\pi_{*} V\right) \mid \operatorname{init}_{\prec}\left(\operatorname{Res}_{\boldsymbol{d}}(V)\right)$ in $K[\boldsymbol{u}]$, because $\operatorname{Res}_{\boldsymbol{d}}\left(\pi_{*} V\right)$ is a power of an irreducible polynomial of positive degree in $\boldsymbol{u}_{0}$ and $r>0$.

For a general $K$-cycle of pure dimension $r$, the statement follows by applying this result to its irreducible components and using the multiplicativity of the order $\prec$. This concludes the inductive step.

REMARK 1.42. - In the projective case $(m=1)$, this result can be alternatively derived from [25, Prop. 4.1], see also [19, Lem. 2.6].

Resultants corresponding to general linear forms play an important role in the definition and study of mixed heights of cycles. We introduce a convenient notation for handling this particular case. Given $\boldsymbol{c}=\left(c_{1}, \ldots, c_{m}\right) \in \mathbb{N}^{m}$ we set

$$
\boldsymbol{e}(\boldsymbol{c})=(\overbrace{\boldsymbol{e}_{1}, \ldots, \boldsymbol{e}_{1}}^{c_{1}}, \ldots, \overbrace{\boldsymbol{e}_{m}, \ldots, \boldsymbol{e}_{m}}^{c_{m}}) \in\left(\mathbb{N}^{m} \backslash\{0\}\right)^{|\boldsymbol{c}|},
$$

where $\boldsymbol{e}_{i}$ denotes the $i$-th vector of the standard basis of $\mathbb{R}^{m}$. For $\boldsymbol{c} \in \mathbb{N}_{r+1}^{m}$, both $\operatorname{Elim}_{\boldsymbol{e}(\boldsymbol{c})}(V)$ and $\operatorname{Res}_{\boldsymbol{e}(\boldsymbol{c})}(V)$ are polynomials in the coefficients of the $r+1$ general linear forms $L_{0}, \ldots, L_{r}$ corresponding to the index $\boldsymbol{e}(\boldsymbol{c})$. In this case, Proposition 1.32 implies that, for $0 \leq i \leq r$,

$$
\operatorname{deg}_{\boldsymbol{u}_{i}}\left(\operatorname{Res}_{\boldsymbol{e}(\boldsymbol{c})}(X)\right)=\operatorname{deg}_{\boldsymbol{c}-\boldsymbol{e}_{j(i)}}(X),
$$

where $j(i)$ is the index $j$ such that $c_{1}+\cdots+c_{j-1}<i+1 \leq c_{1}+\cdots+c_{j}$. 
Proposition 1.45. - Let $m_{i} \in \mathbb{Z}_{>0}, \boldsymbol{n}_{i} \in \mathbb{N}^{m_{i}}$ and $X_{i} \in Z_{r_{i}}\left(\mathbb{P}_{K}^{\boldsymbol{n}_{i}}\right)$ for $i=1,2$. Let $\boldsymbol{c}_{i} \in \mathbb{N}^{m_{i}}$ such that $\left|\boldsymbol{c}_{1}\right|+\left|\boldsymbol{c}_{2}\right|=r_{1}+r_{2}+1$. Then there exists $\lambda \in A^{\times}$such that

$$
\operatorname{Res}_{\boldsymbol{e}\left(\boldsymbol{c}_{1}, \boldsymbol{c}_{2}\right)}\left(X_{1} \times X_{2}\right)=\left\{\begin{array}{cl}
\lambda \operatorname{Res}_{\boldsymbol{e}\left(\boldsymbol{c}_{1}\right)}\left(X_{1}\right)^{\operatorname{deg}_{c_{2}}\left(X_{2}\right)} & \text { if }\left|\boldsymbol{c}_{1}\right|=r_{1}+1,\left|\boldsymbol{c}_{2}\right|=r_{2}, \\
\lambda \operatorname{Res}_{\boldsymbol{e}\left(\boldsymbol{c}_{2}\right)}\left(X_{2}\right)^{\operatorname{deg}_{\boldsymbol{c}_{1}}\left(X_{1}\right)} & \text { if }\left|\boldsymbol{c}_{1}\right|=r_{1},\left|\boldsymbol{c}_{2}\right|=r_{2}+1, \\
1 & \text { otherwise. }
\end{array}\right.
$$

Proof. - We first prove the statement for the case when $K$ is algebraically closed and $A=K$. Let $\boldsymbol{u}=\left\{\boldsymbol{u}_{0}, \ldots, \boldsymbol{u}_{r_{1}+r_{2}}\right\}$ be the group of variables associated to the index $\boldsymbol{e}\left(\boldsymbol{c}_{1}, \boldsymbol{c}_{2}\right)$. By (1.44), for each $0 \leq i \leq r_{1}+r_{2}$ there is $j, 1 \leq j \leq m_{1}+m_{2}$, such that

$$
\operatorname{deg}_{\boldsymbol{u}_{i}}\left(\operatorname{Res}_{\boldsymbol{e}\left(\boldsymbol{c}_{1}, \boldsymbol{c}_{2}\right)}\left(X_{1} \times X_{2}\right)\right)=\operatorname{deg}_{\left(\boldsymbol{c}_{1}, \boldsymbol{c}_{2}\right)-\boldsymbol{e}_{j}}\left(X_{1} \times X_{2}\right) .
$$

If either $\left|\boldsymbol{c}_{1}\right| \geq r_{1}+2$ or $\left|\boldsymbol{c}_{2}\right| \geq r_{2}+2$, then $\operatorname{deg}_{\left(\boldsymbol{c}_{1}, \boldsymbol{c}_{2}\right)-\boldsymbol{e}_{j}}\left(X_{1} \times X_{2}\right)=0$ for all $j$, thanks to Proposition 1.19. Hence all partial degrees are 0 and $\operatorname{Res}_{\boldsymbol{e}\left(\boldsymbol{c}_{1}, \boldsymbol{c}_{2}\right)}\left(X_{1} \times X_{2}\right)=1$.

Consider then the case when $\left|\boldsymbol{c}_{1}\right|=r_{1}+1,\left|\boldsymbol{c}_{2}\right|=r_{2}$. Again by (1.44), for $1 \leq i \leq r_{2}$, there exists $j>m_{1}$ such that

$$
\operatorname{deg}_{\boldsymbol{u}_{r_{1}+i}}\left(\operatorname{Res}_{\boldsymbol{e}\left(\boldsymbol{c}_{1}, \boldsymbol{c}_{2}\right)}\left(X_{1} \times X_{2}\right)\right)=\operatorname{deg}_{\left(\boldsymbol{c}_{1}, \boldsymbol{c}_{2}\right)-\boldsymbol{e}_{j}}\left(X_{1} \times X_{2}\right) .
$$

By Proposition 1.19, this mixed degree also vanishes, because $\left|\boldsymbol{c}_{1}\right|>r_{1}$. Therefore

$$
\operatorname{Res}_{\boldsymbol{e}\left(\boldsymbol{c}_{1}, \boldsymbol{c}_{2}\right)}\left(X_{1} \times X_{2}\right) \in K\left[\boldsymbol{u}_{0}, \ldots, \boldsymbol{u}_{r_{1}}\right] .
$$

Furthermore, suppose that $\operatorname{deg}_{c_{2}}\left(X_{2}\right)=0$. In this case, for each $0 \leq i \leq r_{1}$ there exists $j$, $1 \leq j \leq m_{1}$, such that

$$
\operatorname{deg}_{\boldsymbol{u}_{i}}\left(\operatorname{Res}_{\boldsymbol{e}\left(\boldsymbol{c}_{1}, \boldsymbol{c}_{2}\right)}\left(X_{1} \times X_{2}\right)\right)=\operatorname{deg}_{\left(\boldsymbol{c}_{1}, \boldsymbol{c}_{2}\right)-\boldsymbol{e}_{j}}\left(X_{1} \times X_{2}\right)=\operatorname{deg}_{\boldsymbol{c}_{1}-\boldsymbol{e}_{j}}\left(X_{1}\right) \operatorname{deg}_{\boldsymbol{c}_{2}}\left(X_{2}\right)=0 .
$$

Hence, $\operatorname{Res}_{\boldsymbol{e}\left(\boldsymbol{c}_{1}, \boldsymbol{c}_{2}\right)}\left(X_{1} \times X_{2}\right)=1=\operatorname{Res}_{\boldsymbol{e}\left(\boldsymbol{c}_{1}\right)}\left(X_{1}\right)^{\operatorname{deg}_{c_{2}}\left(X_{2}\right)}$ and the statement holds in this case. Therefore, we assume that $\operatorname{deg}_{c_{2}}\left(X_{2}\right) \neq 0$. By linearity, it suffices to prove the statement for two irreducible varieties $V_{i} \subset \mathbb{P}^{\boldsymbol{n}_{i}}$ of dimension $r_{i}, i=1,2$. We consider first the case when $r_{2}=0$, that is, when $V_{2}=\{\boldsymbol{\xi}\}$ is a point. Then

$$
\mathcal{M}_{\boldsymbol{e}\left(\boldsymbol{c}_{1}\right)}\left(V_{1} \times V_{2}\right)=\mathcal{M}_{\boldsymbol{e}\left(\boldsymbol{c}_{1}\right)}\left(V_{1}\right) \otimes_{K} K\left[\boldsymbol{x}_{2}\right] / I(\boldsymbol{\xi}) .
$$

Hence, for $\boldsymbol{\delta}_{i} \in \mathbb{N}^{m_{i}}$,

$$
\mathcal{M}_{\boldsymbol{e}\left(\boldsymbol{c}_{1}\right)}\left(V_{1} \times V_{2}\right)_{\boldsymbol{\delta}_{1}, \boldsymbol{\delta}_{2}}=\mathcal{M}_{\boldsymbol{e}\left(\boldsymbol{c}_{1}\right)}\left(V_{1}\right)_{\boldsymbol{\delta}_{1}} \otimes_{K}\left(K\left[\boldsymbol{x}_{2}\right] / I(\boldsymbol{\xi})\right)_{\boldsymbol{\delta}_{2}} \simeq \mathcal{M}_{\boldsymbol{e}\left(\boldsymbol{c}_{1}\right)}\left(V_{1}\right)_{\boldsymbol{\delta}_{1}} .
$$

By the definition of the resultant, there exists $\lambda \in K^{\times}$such that

$$
\operatorname{Res}_{\boldsymbol{e}\left(\boldsymbol{c}_{1}\right)}\left(V_{1} \times V_{2}\right)=\lambda \operatorname{Res}_{\boldsymbol{e}\left(\boldsymbol{c}_{1}\right)}\left(V_{1}\right)=\lambda \operatorname{Res}_{\boldsymbol{e}\left(\boldsymbol{c}_{1}\right)}\left(V_{1}\right)^{\operatorname{deg}_{\mathbf{0}}\left(V_{2}\right)},
$$

which proves the statement in this case.

Now let $V_{2} \subset \mathbb{P}^{\boldsymbol{n}_{2}}$ be an irreducible variety of dimension $r_{2} \geq 1$. Write $\boldsymbol{u}^{\prime}=\left(\boldsymbol{u}_{0}, \ldots, \boldsymbol{u}_{r_{1}}\right)$ and let $\ell_{i} \in K\left[\boldsymbol{x}_{2}\right], 1 \leq i \leq r_{2}$, be generic linear forms associated to $\boldsymbol{e}\left(\boldsymbol{c}_{2}\right)$ and $\pi_{2}$ the 
projection $\mathbb{P}^{\boldsymbol{n}_{1}} \times \mathbb{P}^{\boldsymbol{n}_{2}} \rightarrow \mathbb{P}^{\boldsymbol{n}_{2}}$. By (1.46), $\operatorname{Res}_{\boldsymbol{e}\left(\boldsymbol{c}_{1}, \boldsymbol{c}_{2}\right)}\left(V_{1} \times V_{2}\right)$ does not depend on the groups of variables $\boldsymbol{u}_{r_{1}+i}$ for $1 \leq i \leq r_{2}$. Hence,

$$
\begin{aligned}
\operatorname{Res}_{\boldsymbol{e}\left(\boldsymbol{c}_{1}, \boldsymbol{c}_{2}\right)}\left(V_{1} \times V_{2}\right)(\boldsymbol{u}) & =\operatorname{Res}_{\boldsymbol{e}\left(\boldsymbol{c}_{1}, \boldsymbol{c}_{2}\right)}\left(V_{1} \times V_{2}\right)\left(\boldsymbol{u}^{\prime}, \ell_{1}, \ldots, \ell_{r_{2}}\right) \\
& =\lambda \operatorname{Res}_{\boldsymbol{e}\left(\boldsymbol{c}_{1}\right)}\left(\left(V_{1} \times V_{2}\right) \cdot \prod_{i=1}^{r_{2}} \pi_{2}^{*} \operatorname{div}\left(\ell_{i}\right)\right)\left(\boldsymbol{u}^{\prime}\right) \\
& =\lambda \operatorname{Res}_{\boldsymbol{e}\left(\boldsymbol{c}_{1}\right)}\left(V_{1} \times\left(V_{2} \cdot \prod_{i=1}^{r_{2}} \operatorname{div}\left(\ell_{i}\right)\right)\right)\left(\boldsymbol{u}^{\prime}\right)
\end{aligned}
$$

with $\lambda \in K^{\times}$, thanks to Proposition 1.40. The cycle $V_{2} \cdot \prod_{i=1}^{r_{2}} \operatorname{div}\left(\ell_{i}\right)$ is of dimension 0 and so we are in the hypothesis of the previous case. By Corollary 1.14, it is a cycle of degree $\operatorname{deg}_{\boldsymbol{c}_{2}}\left(V_{2}\right)$. Therefore, $\operatorname{Res}_{\boldsymbol{e}\left(\boldsymbol{c}_{1}\right)}\left(V_{1} \times\left(V_{2} \cdot \prod_{i=1}^{r_{2}} \operatorname{div}\left(\ell_{i}\right)\right)\right)=\lambda^{\prime} \operatorname{Res}_{\boldsymbol{e}\left(\boldsymbol{c}_{1}\right)}\left(V_{1}\right)^{\operatorname{deg}_{\boldsymbol{c}_{2}}\left(V_{2}\right)}$ with $\lambda^{\prime} \in K^{\times}$, which completes the proof for the case when $K$ is algebraically closed and $A=K$.

The case of an arbitrary field $K$ which is the field of fractions of a factorial ring $A$ follows from Proposition 1.28 and the fact that the resultants of $V_{1}, V_{2}$ and $V_{1} \times V_{2}$ are primitive polynomials in $A[\boldsymbol{u}]$.

\section{Heights of cycles of multiprojective spaces}

\subsection{Mixed heights of cycles over function fields}

Throughout this section, we denote by $k$ a field and $t=\left\{t_{1}, \ldots, t_{p}\right\}$ a group of variables. The height of a polynomial $f=\sum_{\boldsymbol{a}} \alpha_{\boldsymbol{a}} x_{1}^{a_{1}} \cdots x_{n}^{a_{n}} \in k[\boldsymbol{t}]\left[x_{1}, \ldots, x_{n}\right] \backslash\{0\}$ is defined as

$$
\mathrm{h}(f)=\operatorname{deg}_{\boldsymbol{t}}(f)=\max _{\boldsymbol{a}} \operatorname{deg}\left(\alpha_{\boldsymbol{a}}\right) .
$$

For $f=0$, we set $\mathrm{h}(f)=0$. The following lemma estimates the behavior of the height of polynomials with respect to addition, multiplication and composition. Its proof follows directly from the definitions.

Lemma 2.1. - Let $f_{1}, \ldots, f_{s} \in k[\boldsymbol{t}]\left[x_{1}, \ldots, x_{n}\right]$ and $g \in k[\boldsymbol{t}]\left[y_{1}, \ldots, y_{s}\right]$. Then

1. $\mathrm{h}\left(\sum_{i} f_{i}\right) \leq \max _{i} \mathrm{~h}\left(f_{i}\right)$;

2. $\mathrm{h}\left(\prod_{i} f_{i}\right)=\sum_{i} \mathrm{~h}\left(f_{i}\right)$;

3. $\mathrm{h}\left(g\left(f_{1}, \ldots, f_{s}\right)\right) \leq \mathrm{h}(g)+\operatorname{deg}_{\boldsymbol{y}}(g) \max _{i} \mathrm{~h}\left(f_{i}\right)$.

In the sequel, we extend this notion to cycles of $\mathbb{P}_{k(t)}^{n}$ and study its basic properties. To this end, we specialize the theory in $\S 1$ to the case when the factorial ring $A$ is the polynomial ring $k[\boldsymbol{t}]$ with field of fractions $K=k(\boldsymbol{t})$. In particular, the resultant of an effective equidimensional $k(\boldsymbol{t})$-cycle is a primitive polynomial in $k[\boldsymbol{t}][\boldsymbol{u}]$.

Definition 2.2. - Let $V \subset \mathbb{P}_{k(t)}^{n}$ be an irreducible $k(\boldsymbol{t})$-variety of dimension $r, \boldsymbol{c} \in \mathbb{N}_{r+1}^{m}$ and $\boldsymbol{e}(\boldsymbol{c})$ as in (1.43). The (mixed) height of $V$ of index $\boldsymbol{c}$ is defined as

$$
\mathrm{h}_{\boldsymbol{c}}(V)=\mathrm{h}\left(\operatorname{Res}_{\boldsymbol{e}(\boldsymbol{c})}(V)\right)=\operatorname{deg}_{\boldsymbol{t}}\left(\operatorname{Res}_{\boldsymbol{e}(\boldsymbol{c})}(V)\right) .
$$

This definition extends by linearity to cycles in $Z_{r}\left(\mathbb{P}_{k(t)}^{\boldsymbol{n}}\right)$.

For $n \in \mathbb{N}$ and $X \in Z_{r}\left(\mathbb{P}_{k(t)}^{n}\right)$, the height of $X$ is defined as $\mathrm{h}(X)=\mathrm{h}_{r+1}(X)$. 
Definition 2.3. - Let $\eta$ be an indeterminate. The extended Chow ring of $\mathbb{P}_{k(t)}^{n}$ is the graded ring

$$
A^{*}\left(\mathbb{P}_{k(t)}^{\boldsymbol{n}} ; k[\boldsymbol{t}]\right)=A^{*}\left(\mathbb{P}_{k(t)}^{\boldsymbol{n}}\right) \otimes_{\mathbb{Z}} \mathbb{Z}[\eta] /\left(\eta^{2}\right) \simeq \mathbb{Z}\left[\eta, \theta_{1}, \ldots, \theta_{m}\right] /\left(\eta^{2}, \theta_{1}^{n_{1}+1}, \ldots, \theta_{m}^{n_{m}+1}\right),
$$

where $\theta_{i}$ denotes the class in $A^{*}\left(\mathbb{P}_{k(t)}^{n}\right)$ of the inverse image of a hyperplane of $\mathbb{P}_{k(t)}^{n_{i}}$ under the projection $\mathbb{P}_{k(t)}^{n} \rightarrow \underset{k(t)}{n_{i}}$. For short, we alternatively denote this ring as $A^{*}\left(\mathbb{P}^{\boldsymbol{n}} ; k[\boldsymbol{t}]\right)$. To a cycle $X \in Z_{r}\left(\mathbb{P}_{k(t)}^{n}\right)$ we associate an element of this ring, namely

$$
[X]_{k[t]}=\sum_{\boldsymbol{c} \in \mathbb{N}_{r+1}^{m}, \boldsymbol{c} \leq \boldsymbol{n}} \mathrm{h}_{\boldsymbol{c}}(X) \eta \theta_{1}^{n_{1}-c_{1}} \cdots \theta_{m}^{n_{m}-c_{m}}+\sum_{\boldsymbol{b} \in \mathbb{N}_{r}^{m}, \boldsymbol{b} \leq \boldsymbol{n}} \operatorname{deg}_{\boldsymbol{b}}(X) \theta_{1}^{n_{1}-b_{1}} \cdots \theta_{m}^{n_{m}-b_{m}} .
$$

This is a homogeneous element of degree $|\boldsymbol{n}|-r$.

There is an inclusion of the Chow ring into the extended Chow ring

$$
\imath: A^{*}\left(\mathbb{P}_{k(t)}^{\boldsymbol{n}}\right) \hookrightarrow A^{*}\left(\mathbb{P}_{k(t)}^{\boldsymbol{n}} ; k[\boldsymbol{t}]\right)
$$

satisfying $[X]_{k[t]} \equiv \imath([X])(\bmod \eta)$. In particular, the class of a cycle in the Chow ring is determined by its class in the extended Chow ring.

For a cycle $X$ of pure dimension $r$, we will see in Theorem 2.18(2) that $\mathrm{h}_{\boldsymbol{c}}(X)=0$ for every $c$ such that $c_{i}>n_{i}$ for some $i$. Hence $[X]_{k[t]}$ contains the information about all mixed degrees and heights, since $\left\{\boldsymbol{\theta}^{\boldsymbol{a}}, \eta \boldsymbol{\theta}^{\boldsymbol{a}}\right\}_{\boldsymbol{a} \leq \boldsymbol{n}}$ is a $\mathbb{Z}$-basis of $A^{*}\left(\mathbb{P}^{\boldsymbol{n}} ; k[\boldsymbol{t}]\right)$.

The mixed heights of a $k(\boldsymbol{t})$-cycle $X$ can be interpreted as some mixed degrees of a model of $X$ over $\mathbb{P}_{k}^{p}$. For simplicity, we will only consider the case of projective $k(t)$-cycles, where $t$ is a single variable.

Definition 2.4. - Let $n \in \mathbb{N}$ and $V \subset \mathbb{P}_{k(t)}^{n}$ be an irreducible $k(t)$-variety. Let $\boldsymbol{s}=\left\{s_{0}, s_{1}\right\}$ and $\boldsymbol{x}=\left\{x_{0}, \ldots, x_{n}\right\}$ be groups of variables and $\mathscr{f} \subset k[\boldsymbol{s}, \boldsymbol{x}]$ the bihomogeneous ideal generated by all the polynomials of the form $s_{0}^{\operatorname{deg}_{t}(f)} f\left(s_{1} / s_{0}, \boldsymbol{x}\right)$ for $f \in I(V) \cap k[t, \boldsymbol{x}]$. The standard model of $V$ over $\mathbb{P}_{k}^{1}$ is defined as the $k$-variety $V(\mathcal{J}) \subset \mathbb{P}_{k}^{1} \times \mathbb{P}_{k}^{n}$. This notion extends by linearity to cycles of $\mathbb{P}_{k(t)}^{n}:$ the standard model of a cycle $X=\sum_{V} m_{V} V$ is defined as $\chi=\sum_{V} m_{V} V$, where $V$ denotes the standard model of the irreducible $k(t)$-variety $V$.

REMARK 2.5. - Set $V_{0}$ and $V_{\infty}$ for the restriction of $V$ to the open subsets $\left(\mathbb{P}_{k}^{1} \backslash\{(0: 1)\}\right) \times \mathbb{P}_{k}^{n}$ and $\left(\mathbb{P}_{k}^{1} \backslash\{(1: 0)\}\right) \times \mathbb{P}_{k}^{n}$, respectively. These are irreducible $k$-varieties which correspond to the prime ideals $\mathscr{I}_{0}=I(V) \cap k[t][\boldsymbol{x}]$ and $\mathscr{I}_{\infty}=I(V) \cap k\left[t^{-1}\right][\boldsymbol{x}]$, respectively, and form a covering of $V$. In particular, $V$ is an irreducible $k$-variety.

A variety $W \subset \mathbb{P}_{k}^{1} \times \mathbb{P}_{k}^{n}$ is vertical if its projection to $\mathbb{P}_{k}^{1}$ consists in a single point. The following lemma shows that the standard model of a cycle of $\mathbb{P}_{k(t)}^{n}$ of pure dimension $r \geq 0$ is a cycle of $\mathbb{P}_{k}^{1} \times \mathbb{P}_{k}^{n}$ without vertical components. Moreover, there is a bijection between $Z_{r}\left(\mathbb{P}_{k(t)}^{n}\right)$ and the set of cycles in $Z_{r+1}\left(\mathbb{P}_{k}^{1} \times \mathbb{P}_{k}^{n}\right)$ without vertical components.

Lemma 2.6. - Let $n \in \mathbb{N}$ and $r \geq 0$.

1. Let $X \in Z_{r}\left(\mathbb{P}_{k(t)}^{n}\right)$. Then its standard model $\chi$ is a cycle of $\mathbb{P}_{k}^{1} \times \mathbb{P}_{k}^{n}$ of pure dimension $r+1$, without vertical fibers, and the generic fiber of $\chi \rightarrow \mathbb{P}_{k}^{1}$ coincides with $X$ under the natural identification of the generic fiber of $\mathbb{P}_{k}^{1} \times \mathbb{P}_{k}^{n} \rightarrow \mathbb{P}_{k}^{1}$ with $\mathbb{P}_{k(t)}^{n}$. 
2. Let $Y \in Z_{r+1}\left(\mathbb{P}_{k}^{1} \times \mathbb{P}_{k}^{n}\right)$ be a cycle without vertical components and $Y$ the generic fiber of $\mathcal{Y} \rightarrow \mathbb{P}_{k}^{1}$. Then $Y \in Z_{r}\left(\mathbb{P}_{k(t)}^{n}\right)$ and $Y$ is its standard model.

Proof. - (1) It suffices to prove the statement for an irreducible variety $V \subset \mathbb{P}_{k(t)}^{n}$. We keep the notation in Definition 2.4 and Remark 2.5 and we denote by $\pi$ the projection $V \rightarrow \mathbb{P}_{k}^{1}$. Suppose that $V$ is vertical. This would imply that $\pi\left(V_{0}\right)$ is a point. Hence, there exists $f \in k[t] \backslash\{0\}$ such that $\pi\left(\mathcal{V}_{0}\right) \subset V(f)$ or, equivalently, such that $f \in I_{0}$. But this would imply that $1 \in I(V)$ and a fortiori, $V=\varnothing$. This is contrary to our assumptions and so we deduce that $V$ is not vertical.

Choose $0 \leq i \leq n$ such that $V$ is not contained in $V\left(x_{i}\right)$. For simplicity, we suppose that $i=0$. Set $V_{x_{0}}=V \backslash V\left(x_{0}\right) \subset \mathbb{P}_{k(t)}^{n} \backslash V\left(x_{0}\right) \simeq \mathbb{A}_{k(t)}^{n}$. Set $x_{i}^{\prime}=x_{i} / x_{0}, 1 \leq i \leq n$, and let $J \subset k(t)\left[x_{1}^{\prime}, \ldots, x_{n}^{\prime}\right]$ be the ideal of $V_{x_{0}}$. Then $J \cap k\left[t, x_{1}^{\prime}, \ldots, x_{n}^{\prime}\right]$ is the ideal of the $k$-variety $\left(V_{0}\right)_{x_{0}}:=V_{0} \backslash V\left(x_{0}\right) \subset\left(\mathbb{A}_{k}^{1} \times \mathbb{P}_{k}^{n}\right) \backslash V\left(x_{0}\right) \simeq \mathbb{A}_{k}^{1} \times \mathbb{A}_{k}^{n}$. Hence,

$$
\left(V_{0}\right)_{x_{0}} \times_{\mathbb{A}_{k}^{1}} \operatorname{Spec}(k(t))=\operatorname{Spec}\left(k\left[t, \boldsymbol{x}^{\prime}\right] / I\left(\left(V_{0}\right)_{x_{0}}\right) \otimes_{k} k(t)\right)=\operatorname{Spec}\left(k(t)\left[\boldsymbol{x}^{\prime}\right] / J\right)=V_{x_{0}} .
$$

The generic fiber of $\pi$ coincides with the closure in $\mathbb{P}_{k(t)}^{n}$ of the generic fiber of $\left(V_{0}\right)_{x_{0}} \rightarrow \mathbb{A}_{k}^{1}$, namely, with $\overline{V_{x_{0}}}=V$. The fact that $\pi$ is surjective with generic fiber $V$ implies that $\operatorname{dim}(V)=\operatorname{dim}(V)+\operatorname{dim}\left(\mathbb{P}_{k}^{1}\right)=r+1$ by the theorem of dimension of fibers.

(2) It is sufficient to consider the case of an irreducible $k$-variety $W \subset \mathbb{P}_{k}^{1} \times \mathbb{P}_{k}^{n}$ without vertical components. Let $\mathcal{J}_{0} \subset k[t][\boldsymbol{x}]$ be the ideal of the restriction of $W$ to the open subset $\left(\mathbb{P}_{k}^{1} \backslash\{(0: 1)\}\right) \times \mathbb{P}_{k}^{n} \simeq \mathbb{A}_{k}^{1} \times \mathbb{P}_{k}^{n}$. It is a prime ideal of Krull dimension $r+2$ and $\mathscr{J}_{0} \cap k[t]=\{0\}$ since $\pi$ is surjective. Hence, $J:=k(t) \otimes_{k[t]} \mathscr{J}_{0}$ is a homogeneous prime ideal of Krull dimension $r+1$ which defines the generic fiber of $\pi$. Moreover, $\mathcal{g}_{0}=J \cap k[t][\boldsymbol{x}]$ and so $W$ is the standard model of $W$.

Lemma 2.7. - Let $I \subset k(t)[\boldsymbol{x}]$ be an equidimensional ideal, $X(I) \in Z\left(\mathbb{P}_{k(t)}^{n}\right)$ its associated cycle and $\chi$ the standard model of $X(I)$. Let $I \subset k[\boldsymbol{s}, \boldsymbol{x}]$ be the ideal generated by all the polynomials of the form $s_{0}^{\operatorname{deg}_{t}(f)} f\left(s_{1} / s_{0}, \boldsymbol{x}\right)$ for $f \in I \cap k[t, \boldsymbol{x}]$. Then $\chi=X(\mathcal{J})$.

Proof. - This can be verified by going through the ideals: the minimal primes of $I$ are in bijection with the minimal primes of $\mathcal{I}$, and this bijection preserves multiplicities.

There is an isomorphism $\phi: A^{*}\left(\mathbb{P}_{k(t)}^{n} ; k[t]\right) \rightarrow A^{*}\left(\mathbb{P}_{k}^{1} \times \mathbb{P}_{k}^{n}\right)$ which sends the generators $\eta, \theta_{1} \in A^{*}\left(\mathbb{P}_{k}^{n} ; k[t]\right)$ to the generators $\theta_{1}, \theta_{2} \in A^{*}\left(\mathbb{P}_{k}^{1} \times \mathbb{P}_{k}^{n}\right)$, respectively. Next result shows that, via this isomorphism, the class of a projective $k(t)$-cycle $X$ identifies with the class of its standard model $\chi$. In particular, the height of $X$ coincides with a mixed degree of $\chi$.

Proposition 2.8. - Let $X \in Z_{r}\left(\mathbb{P}_{k(t)}^{n}\right)$ and $\chi$ be the standard model of $X$. Then

$$
\phi\left([X]_{k(t)}\right)=[\chi] .
$$

Equivalently, $\operatorname{deg}(X)=\operatorname{deg}_{1, r}(\chi)$ and $\mathrm{h}(X)=\operatorname{deg}_{0, r+1}(\chi)$.

Proof. - It is enough to prove the statement for an irreducible $k(t)$-variety $V$ with standard model $V$. Set $\boldsymbol{u}=\left\{\boldsymbol{u}_{0}, \ldots, \boldsymbol{u}_{r}\right\}, \mathbf{1}=(1, \ldots, 1) \in \mathbb{N}^{r+1}, \boldsymbol{e}=(1,0)$ and $\widetilde{\boldsymbol{e}}=((0,1), \ldots,(0,1)) \in\left(\mathbb{N}^{2}\right)^{r+1}$. We first claim that there exists $\lambda \in k^{\times}$such that

$$
\operatorname{Res}_{\mathbf{1}}(V)(\boldsymbol{u})=\lambda \operatorname{Res}_{\boldsymbol{e}, \tilde{\boldsymbol{e}}}(V)((-t, 1), \boldsymbol{u}) .
$$


Let $I \subset k(t)[\boldsymbol{x}]$ and $\mathscr{I} \subset k[\boldsymbol{s}, \boldsymbol{x}]$ denote the homogeneous ideal of $V$ and the bihomogeneous ideal of $\mathcal{V}$, respectively. Set

$$
\mathscr{J}_{1}=k(t) \otimes_{k} J+\left(s_{1}-t s_{0}\right), \quad \mathscr{J}_{2}=k(t)[s] \otimes_{k(t)} I+\left(s_{1}-t s_{0}\right) \quad \subset k(t)[\boldsymbol{s}, \boldsymbol{x}] .
$$

These ideals define the subschemes $V_{k(t)} \cap V\left(s_{1}-t s_{0}\right)$ and $\{(1: t)\} \times V$ of $\mathbb{P}_{k(t)}^{1} \times \mathbb{P}_{k(t)}^{n}$, respectively. For $f \in k[t, \boldsymbol{x}]$, set

$$
f^{\text {hom }}=s_{0}^{\operatorname{deg}_{t}(f)} f\left(\frac{s_{1}}{s_{0}}, \boldsymbol{x}\right) \in k[\boldsymbol{s}, \boldsymbol{x}] .
$$

This polynomial is homogeneous of $\operatorname{degree} \operatorname{deg}_{t}(f)$ with respect to the variables $s$. Observe that

$$
f^{\text {hom }} \equiv s_{0}^{\operatorname{deg}_{t}(f)} f \equiv\left(t^{-1} s_{1}\right)^{\operatorname{deg}_{t}(f)} f \quad\left(\bmod \left(s_{1}-t s_{0}\right)\right) .
$$

The ideal $\mathscr{I}$ is generated by $\left\{f^{\text {hom }}: f \in I \cap k[t, \boldsymbol{x}]\right\}$. By (2.10), these generators lie in $\mathscr{J}_{2}$; hence $I \subset \mathscr{J}_{2}$ and so $\mathscr{J}_{1} \subset \mathscr{J}_{2}$. The Equations (2.10) also imply that $I$ is contained in the localization $\left(\mathscr{J}_{2}\right)_{s_{i}} \subset k(t)[\boldsymbol{s}, \boldsymbol{x}]_{s_{i}}$ for $i=0,1$. Hence, $\left(\mathscr{J}_{1}\right)_{s_{i}}=\left(\mathscr{J}_{2}\right)_{s_{i}}$ and so both ideals define the same subschemes of $\mathbb{P}_{k(t)}^{1} \times \mathbb{P}_{k(t)}^{n}$. In particular, $\operatorname{div}\left(s_{1}-t s_{0}\right)$ intersects $V_{k(t)}$ properly and we have the equality of cycles

$$
V_{k(t)} \cdot \operatorname{div}\left(s_{1}-t s_{0}\right)=\{(1: t)\} \times V \in Z_{r}\left(\mathbb{P}_{k(t)}^{1} \times \mathbb{P}_{k(t)}^{n}\right)
$$

Applying successively Propositions 1.28 and 1.40, (2.11) and Proposition 1.45, we obtain that there exists $\mu_{0}, \mu \in k[t] \backslash\{0\}$ such that

$$
\begin{array}{r}
\operatorname{Res}_{\boldsymbol{e}, \widetilde{\boldsymbol{e}}}(V)((-t, 1), \boldsymbol{u})=\mu_{0} \operatorname{Res}_{\widetilde{\boldsymbol{e}}}\left(V_{k(t)} \cdot \operatorname{div}\left(s_{1}-t s_{0}\right)\right)(\boldsymbol{u}) \\
=\mu_{0} \operatorname{Res}_{\tilde{\boldsymbol{e}}}(\{(1: t)\} \times V)(\boldsymbol{u})=\mu \operatorname{Res}_{\mathbf{1}}(V)(\boldsymbol{u}) .
\end{array}
$$

It remains to show that $\mu \in k^{\times}$. For $\tau \in \bar{k}$, $\operatorname{div}\left(s_{1}-\tau s_{0}\right)$ intersects $V$ properly since the projection $\pi: \mathcal{V} \rightarrow \mathbb{P}_{k}^{1}$ is surjective (Lemma 2.6). Proposition 1.40 then implies that $\operatorname{Res}_{\boldsymbol{e}, \tilde{\boldsymbol{e}}}(V)((-\tau, 1), \boldsymbol{u}) \neq 0$. Furthermore, $\left.\operatorname{Res}_{\mathbf{1}}(V)(\boldsymbol{u})\right|_{t=\tau} \neq 0$ as this resultant is a primitive polynomial in $k[t][\boldsymbol{u}]$. Specializing (2.12) at $t=\tau$, we deduce that $\mu(\tau) \neq 0$ for all $\tau \in \bar{k}$. Hence, $\mu \in k^{\times}$and (2.9) follows for $\lambda=\mu^{-1}$.

Let $\boldsymbol{v}=\left\{v_{0}, v_{1}\right\}$ be a group of variables. By (2.9), for any $0 \leq i \leq r$,

$$
\operatorname{deg}_{\boldsymbol{u}_{i}}\left(\operatorname{Res}_{\mathbf{1}}(V)(\boldsymbol{u})\right)=\operatorname{deg}_{\boldsymbol{u}_{i}}\left(\operatorname{Res}_{\boldsymbol{e}, \tilde{\boldsymbol{e}}}(V)((-t, 1), \boldsymbol{u})\right)=\operatorname{deg}_{\boldsymbol{u}_{i}}\left(\operatorname{Res}_{\boldsymbol{e}, \tilde{\boldsymbol{e}}}(V)(\boldsymbol{v}, \boldsymbol{u})\right),
$$

since $\operatorname{Res}_{\boldsymbol{e}, \tilde{\boldsymbol{e}}}(\mathcal{V})$ is homogeneous in the variables $\boldsymbol{v}$. Proposition 1.32 then implies that $\operatorname{deg}(V)=\operatorname{deg}_{1, r}(V)$.

Applying an argument similar to the one above, we verify that $s_{0}$ intersects $V$ properly. Hence, $\operatorname{Res}_{\boldsymbol{e}, \tilde{\boldsymbol{e}}}(\mathcal{V})((1,0), \boldsymbol{u})=\lambda \operatorname{Res}_{\tilde{e}}\left(\mathcal{V} \cdot \operatorname{div}\left(s_{0}\right)\right)(\boldsymbol{u})$ with $\lambda \in k^{\times}$. In particular, this specialization is not zero, and so the degrees of $\operatorname{Res}_{\boldsymbol{e}, \tilde{\boldsymbol{e}}}(\mathcal{V})$ in $v_{0}$ and in $\boldsymbol{v}$ coincide. Therefore,

$$
\mathrm{h}(V)=\operatorname{deg}_{t}\left(\operatorname{Res}_{\mathbf{1}}(V)(\boldsymbol{u})\right)=\operatorname{deg}_{t}\left(\operatorname{Res}_{\boldsymbol{e}, \tilde{\boldsymbol{e}}}(V)((-t, 1), \boldsymbol{u})\right)=\operatorname{deg}_{\boldsymbol{v}}\left(\operatorname{Res}_{\boldsymbol{e}, \tilde{\boldsymbol{e}}}(V)(\boldsymbol{v}, \boldsymbol{u})\right) .
$$

By Proposition 1.32, we conclude that $\mathrm{h}(V)=\operatorname{deg}_{0, r+1}(\mathcal{V})$.

Next proposition collects some basic properties of mixed heights and classes in the extended Chow ring of multiprojective cycles over $k(\boldsymbol{t})$ with $\boldsymbol{t}=\left\{t_{1}, \ldots, t_{p}\right\}$. 
Proposition 2.13. - $\quad$ 1. Let $X \in Z_{r}^{+}\left(\mathbb{P}_{k(t)}^{\boldsymbol{n}}\right)$. Then $[X]_{k[t]} \geq 0$. In particular, $\mathrm{h}_{\boldsymbol{c}}(X) \geq 0$ for all $\boldsymbol{c} \in \mathbb{N}_{r+1}^{m}$.

2. Let $X \in Z_{r}\left(\mathbb{P}_{k}^{n}\right)$. Then $\left[X_{k(t)}\right]_{k[t]}=\imath\left(\left[X_{k(t)}\right]\right)$ or equivalently, $\mathrm{h}_{\boldsymbol{c}}(X)=0$ for all $\boldsymbol{c} \in \mathbb{N}_{r+1}^{m}$. In particular, $\left[\mathbb{P}_{k(t)}^{\boldsymbol{n}}\right]_{k[t]}=1$.

3. Let $\boldsymbol{\xi}=\left(\boldsymbol{\xi}_{1}, \ldots, \boldsymbol{\xi}_{m}\right) \in \mathbb{P}^{\boldsymbol{n}}$ be a point with coordinates in $k(\boldsymbol{t})$ and for each $1 \leq i \leq m$ write $\boldsymbol{\xi}_{i}=\left(\xi_{i, j}\right)_{j}$ for coprime polynomials $\xi_{i, j} \in k[\boldsymbol{t}]$. Then

$$
[\boldsymbol{\xi}]_{k[t]}=\sum_{i=1}^{m} \mathrm{~h}\left(\boldsymbol{\xi}_{i}\right) \eta \boldsymbol{\theta}^{\boldsymbol{n}-\boldsymbol{e}_{i}}+\boldsymbol{\theta}^{\boldsymbol{n}}
$$

with $\mathrm{h}\left(\boldsymbol{\xi}_{i}\right):=\max _{j} \mathrm{~h}\left(\xi_{i, j}\right)=\max _{j} \operatorname{deg}\left(\xi_{i, j}\right)$. In particular, $\mathrm{h}_{\boldsymbol{e}_{i}}(\boldsymbol{\xi})=\mathrm{h}\left(\boldsymbol{\xi}_{i}\right)$.

4. Let $D \in \operatorname{Div}^{+}\left(\mathbb{P}_{k(t)}^{\boldsymbol{n}}\right)$ and $f_{D} \in k[\boldsymbol{t}][\boldsymbol{x}]$ its primitive defining polynomial. Then

$$
[D]_{k[t]}=\mathrm{h}\left(f_{D}\right) \eta+\sum_{i=1}^{m} \operatorname{deg}_{\boldsymbol{x}_{i}}\left(f_{D}\right) \theta_{i}
$$

In particular, $\mathrm{h}_{\boldsymbol{n}}(D)=\mathrm{h}\left(f_{D}\right)$.

Proof. - (1) This is immediate from the definition of mixed heights and classes in the extended Chow ring.

(2) By Proposition 1.28, there exists $\lambda \in k(\boldsymbol{t})^{\times}$such that $\operatorname{Res}_{\boldsymbol{e}(\boldsymbol{c})}\left(X_{k(\boldsymbol{t})}\right)=\lambda \operatorname{Res}_{\boldsymbol{e}(\boldsymbol{c})}(X)$. Since the term in the left-hand side is primitive with respect to $k[t]$, we deduce that $\lambda \in k^{\times}$. Hence, this resultant does not depend on $\boldsymbol{t}$ and $\mathrm{h}_{\boldsymbol{c}}(X)=0$ for all $\boldsymbol{c}$. This readily implies that $\left[X_{k(t)}\right]_{k[t]}=\imath\left(\left[X_{k(t)}\right]\right)$. The rest of the statement follows from Proposition 1.10(2).

(3) By Proposition 1.10(3), it is enough to show that $\mathrm{h}_{\boldsymbol{e}_{i}}(\boldsymbol{\xi})=\mathrm{h}\left(\boldsymbol{\xi}_{i}\right)$. By Corollary 1.38, there exists $\lambda_{i} \in k(\boldsymbol{t})^{\times}$such that $\operatorname{Res}_{\boldsymbol{e}_{i}}(\boldsymbol{\xi})=\lambda_{i} L_{i}\left(\boldsymbol{\xi}_{i}\right)$, where $L_{i}$ is the general linear form of multidegree $\boldsymbol{e}_{i}$. Indeed, $\lambda_{i} \in k^{\times}$since $\operatorname{Res}_{\boldsymbol{e}_{i}}(\boldsymbol{\xi})$ and $L_{i}\left(\boldsymbol{\xi}_{i}\right)$ are primitive polynomials in $k[\boldsymbol{t}]$. Hence, $\mathrm{h}_{\boldsymbol{e}_{i}}(\boldsymbol{\xi})=\operatorname{deg}_{\boldsymbol{t}}\left(L_{i}\left(\boldsymbol{\xi}_{i}\right)\right)=\mathrm{h}\left(\boldsymbol{\xi}_{i}\right)$.

(4) By Proposition 1.10(4), it is enough to show that $\mathrm{h}_{\boldsymbol{n}}(D)=\mathrm{h}\left(f_{D}\right)$. Set $\boldsymbol{d}=\operatorname{deg}\left(f_{D}\right)$. Consider the general form $F$ of multidegree $\boldsymbol{d}$ and the general linear forms $\boldsymbol{L}=\left(L_{0}, \ldots, L_{|\boldsymbol{n}|-1}\right)$ corresponding to $\boldsymbol{e}(\boldsymbol{n})$. Write for short $R=\operatorname{Res}_{\boldsymbol{e}(\boldsymbol{n}), \boldsymbol{d}}\left(\mathbb{P}_{k}^{\boldsymbol{n}}\right)$. Using Propositions 1.40 and 1.28 , we deduce that there exists $\lambda \in k(\boldsymbol{t})^{\times}$such that

$$
\lambda \operatorname{Res}_{\boldsymbol{e}(\boldsymbol{n})}(D)(\boldsymbol{L})=R\left(\boldsymbol{L}, f_{D}\right) .
$$

Observe that $\lambda \in k[\boldsymbol{t}] \backslash\{0\}$ because the resultant in the left-hand side is a primitive polynomial. On the other hand, let $H \subset \mathbb{A}_{k}^{p}$ be an irreducible $k$-hypersurface. The fact that $f_{D}$ is primitive implies that there exists $\boldsymbol{\xi} \in H$ such that $f_{D}(\boldsymbol{\xi}, \boldsymbol{x}) \neq 0$. Applying Proposition 1.40 to the cycle $\mathbb{P}_{k}^{\boldsymbol{n}}$ and $f_{D}(\boldsymbol{\xi}, \boldsymbol{x}) \in k[\boldsymbol{x}]$ we deduce

$$
R\left(\boldsymbol{L}, f_{D}(\boldsymbol{\xi}, \boldsymbol{x})\right) \neq 0 .
$$

Hence, $\lambda(\boldsymbol{\xi}) \neq 0$. This implies that $V(\lambda)$ contains no hypersurface of $\mathbb{A}_{k}^{p}$ and so $\lambda \in k^{\times}$. Hence,

$$
\mathrm{h}_{\boldsymbol{n}}(D)=\operatorname{deg}_{\boldsymbol{t}}\left(\operatorname{Res}_{\boldsymbol{e}(\boldsymbol{n})}(D)\right)=\operatorname{deg}_{\boldsymbol{t}}\left(R\left(\boldsymbol{L}, f_{D}\right)\right)=\operatorname{deg}_{\boldsymbol{t}}\left(f_{D}\right) \operatorname{deg}_{\boldsymbol{u}_{n}}(R)=\mathrm{h}\left(f_{D}\right),
$$

since $\operatorname{deg}_{\boldsymbol{t}}\left(f_{D}\right)=\mathrm{h}\left(f_{D}\right)$ and, by Propositions 1.32 and $1.10(2), \operatorname{deg}_{\boldsymbol{u}_{n}}(R)=1$. 
We recall some notions and properties of valuations of fields. Let $T_{0}, \ldots, T_{p}$ denote the standard homogeneous coordinates of $\mathbb{P}_{k}^{p}$. If we identify each variable $t_{i}$ with the rational function $T_{i} / T_{0}$, we can regard $k(\boldsymbol{t})$ as the field of rational functions of this projective space: given $\alpha \in k(\boldsymbol{t})$, then $\alpha\left(T_{1} / T_{0}, \ldots, T_{p} / T_{0}\right)$ is homogeneous of degree 0 and defines a rational function on $\mathbb{P}_{k}^{p}$.

Given an irreducible $k$-hypersurface $H$ of $\mathbb{P}^{p}$ and $\alpha \in k(\boldsymbol{t})$, we set $\operatorname{ord}_{H}(\alpha)$ for the order of vanishing of $\alpha$ along $H$. The map ord $\operatorname{or}_{H}: k(\boldsymbol{t}) \rightarrow \mathbb{Z}$ is a valuation of $k(\boldsymbol{t})$. If $H_{\infty}:=V\left(T_{0}\right)$ is the hyperplane at infinity, then $\operatorname{ord}_{H_{\infty}}(\alpha)=-\operatorname{deg}(\alpha)$ where $\operatorname{deg}(\alpha)=\operatorname{deg}\left(\alpha_{1}\right)-\operatorname{deg}\left(\alpha_{2}\right)$ for any $\alpha_{i} \in k[\boldsymbol{t}]$ such that $\alpha=\alpha_{1} / \alpha_{2}$. If $H \neq H_{\infty}$, then $\operatorname{ord}_{H}(\alpha)$ coincides with the order of the polynomial $f_{H}\left(1, t_{1}, \ldots, t_{p}\right)$ in the factorization of $\alpha$, where $f_{H} \in k\left[T_{0}, \ldots, T_{p}\right]$ is the defining polynomial of $H$.

Let $K$ be an arbitrary field and $v$ a valuation of $K$. For a polynomial $f$ over $K$, we set $v(f)$ for the minimum of the valuation of its coefficients. Gauss Lemma states that for any given polynomials $f, g$ over $K$,

$$
v(f g)=v(f)+v(g) .
$$

Given a finite extension $E$ of $K$, there exists a (non-necessarily unique) valuation $w$ of $E$ extending $v$.

The height of an arbitrary resultant can be expressed in terms of mixed heights:

Lemma 2.14. - Let $X \in Z_{r}^{+}\left(\mathbb{P}_{k(t)}^{\boldsymbol{n}}\right)$ and $\boldsymbol{d} \in\left(\mathbb{N}^{m} \backslash\{\mathbf{0}\}\right)^{r+1}$. Then

$$
\operatorname{deg}_{\boldsymbol{t}}\left(\operatorname{Res}_{\boldsymbol{d}}(X)\right)=\operatorname{coeff}_{\eta \boldsymbol{\theta}^{n}}\left([X]_{k[t]} \prod_{i=0}^{r} \sum_{j=1}^{m} d_{i, j} \theta_{j}\right) .
$$

In particular, for $\boldsymbol{b} \in \mathbb{N}_{r}^{m}$ and $\boldsymbol{d}_{r} \in \mathbb{N}^{m} \backslash\{\mathbf{0}\}$,

$$
\operatorname{deg}_{\boldsymbol{t}}\left(\operatorname{Res}_{\boldsymbol{e}(\boldsymbol{b}), \boldsymbol{d}_{r}}(X)\right)=\sum_{j=1}^{m} d_{r, j} \mathrm{~h}_{\boldsymbol{b}+\boldsymbol{e}_{j}}(X) .
$$

Proof. - Write $\boldsymbol{d}=\left(\boldsymbol{d}_{0}, \ldots, \boldsymbol{d}_{r}\right)$ for $\boldsymbol{d}_{i} \in \mathbb{N}^{m} \backslash\{\mathbf{0}\}$. We claim that

$$
\operatorname{deg}_{\boldsymbol{t}}\left(\operatorname{Res}_{\boldsymbol{d}_{0}, \ldots, \boldsymbol{d}_{r}}(X)\right)=\sum_{j=1}^{m} d_{0, j} \operatorname{deg}_{\boldsymbol{t}}\left(\operatorname{Res}_{\boldsymbol{e}_{j}, \boldsymbol{d}_{1}, \ldots, \boldsymbol{d}_{r}}(X)\right) .
$$

We first consider the case $r=0$. Let $X_{\overline{k(t)}}=\sum_{\boldsymbol{\xi}} m_{\boldsymbol{\xi}} \boldsymbol{\xi}$ with $\boldsymbol{\xi} \in \mathbb{P}^{\boldsymbol{n}}$ and $m_{\boldsymbol{\xi}} \geq 1$. For each of these points write $\boldsymbol{\xi}=\left(\xi_{i, j}\right)_{i, j}$ for some $\xi_{i, j} \in \overline{k(\boldsymbol{t})}$. Let $F_{0}$ and $L_{j}$ denote the general forms of degree $\boldsymbol{d}_{0}$ and $\boldsymbol{e}_{j}$ as in (1.21), respectively. By Proposition 1.28 and Corollary 1.38, there exist $\mu, \lambda_{j} \in \overline{k(t)}^{\times}$such that

$$
\operatorname{Res}_{\boldsymbol{d}_{0}}(X)\left(F_{0}\right)=\mu \prod_{\boldsymbol{\xi}} F_{0}(\boldsymbol{\xi})^{m_{\boldsymbol{\xi}}}, \quad \operatorname{Res}_{\boldsymbol{e}_{j}}(X)\left(L_{j}\right)=\lambda_{j} \prod_{\boldsymbol{\xi}} L_{j}\left(\boldsymbol{\xi}_{j}\right)^{m_{\boldsymbol{\xi}}}
$$

Picking a suitable choice of multihomogeneous coordinates for the $\boldsymbol{\xi}$ 's, it is possible to set $\lambda_{j}=1$ for all $j$. We have

$$
\operatorname{Res}_{\boldsymbol{d}_{0}}(X)\left(\prod_{j=1}^{m} L_{j}^{d_{0, j}}\right)=\mu \prod_{\boldsymbol{\xi}} \prod_{j=1}^{m} L_{j}(\boldsymbol{\xi})^{d_{0, j} m_{\boldsymbol{\xi}}}=\mu \prod_{j=1}^{m} \operatorname{Res}_{\boldsymbol{e}_{j}}(X)\left(L_{j}\right)^{d_{0, j}} .
$$


We deduce that $\mu \in k(\boldsymbol{t})^{\times}$since all considered resultants have coefficients in $k[\boldsymbol{t}]$. Now let $E$ be a sufficiently large finite extension of $k(t)$ containing all the chosen coordinates $\xi_{i, j}$. For any valuation $v$ of $E$,

$$
v\left(F_{0}(\boldsymbol{\xi})\right)=\min _{|\boldsymbol{a}|=\boldsymbol{d}_{0}} v\left(\boldsymbol{\xi}^{\boldsymbol{a}}\right)=\sum_{j=1}^{m} d_{0, j} \min _{\ell} v\left(\xi_{j, \ell}\right)=\sum_{j=1}^{m} d_{0, j} v\left(L_{j}\left(\boldsymbol{\xi}_{j}\right)\right) .
$$

Let $H$ be an irreducible $k$-hypersurface of $\mathbb{P}^{p}$ different from $H_{\infty}$, and $v_{H}$ a valuation of $E$ extending $\operatorname{ord}_{H}$. Since $\operatorname{Res}_{\boldsymbol{d}_{0}}(X)$ and $\operatorname{Res}_{\boldsymbol{e}_{j}}(X)$ are primitive with respect to $k[\boldsymbol{t}]$, we have that $v_{H}\left(\operatorname{Res}_{\boldsymbol{d}_{0}}(X)\right)=v_{H}\left(\operatorname{Res}_{\boldsymbol{e}_{j}}(X)\right)=0$. By Gauss Lemma,

$$
\begin{aligned}
\operatorname{ord}_{H}(\mu) & =-\operatorname{ord}_{H}\left(\prod_{\boldsymbol{\xi}} F_{0}(\boldsymbol{\xi})^{m_{\boldsymbol{\xi}}}\right)=-\sum_{\boldsymbol{\xi}} m_{\boldsymbol{\xi}} v_{H}\left(F_{0}(\boldsymbol{\xi})\right), \\
0 & =\operatorname{ord}_{H}\left(\prod_{\boldsymbol{\xi}} L_{j}\left(\boldsymbol{\xi}_{j}\right)^{m_{\boldsymbol{\xi}}}\right)=\sum_{\boldsymbol{\xi}} m_{\boldsymbol{\xi}} v_{H}\left(L_{j}\left(\boldsymbol{\xi}_{j}\right)\right) .
\end{aligned}
$$

Applying (2.17),

$$
\operatorname{ord}_{H}(\mu)=-\sum_{\boldsymbol{\xi}} m_{\boldsymbol{\xi}} v_{H}\left(F_{0}(\boldsymbol{\xi})\right)=-\sum_{j=1}^{m} d_{0, j}\left(\sum_{\boldsymbol{\xi}} m_{\boldsymbol{\xi}} v_{H}\left(L_{j}\left(\boldsymbol{\xi}_{j}\right)\right)\right)=0 .
$$

Since this holds for every $H \neq H_{\infty}$, we deduce that $\mu \in k^{\times}$. Now let $v_{\infty}$ be a valuation of $E$ extending $\operatorname{ord}_{H_{\infty}}$. Applying again Gauss Lemma and (2.17),

$$
\begin{aligned}
\operatorname{ord}_{H_{\infty}}\left(\operatorname{Res}_{\boldsymbol{d}_{0}}(X)\right)=\sum_{\boldsymbol{\xi}} m_{\boldsymbol{\xi}} v_{\infty}\left(F_{0}(\boldsymbol{\xi})\right) \\
=\sum_{j=1}^{m} d_{0, j}\left(\sum_{\boldsymbol{\xi}} m_{\boldsymbol{\xi}} v_{\infty}\left(L_{j}\left(\boldsymbol{\xi}_{j}\right)\right)\right)=\sum_{j=1}^{m} d_{0, j} \operatorname{ord}_{H_{\infty}}\left(\operatorname{Res}_{\boldsymbol{e}_{j}}(X)\right) .
\end{aligned}
$$

Hence $\operatorname{deg}_{\boldsymbol{t}}\left(\operatorname{Res}_{\boldsymbol{d}_{0}}(X)\right)=\sum_{j=1}^{m} d_{0, j} \operatorname{deg}_{\boldsymbol{t}}\left(\operatorname{Res}_{\boldsymbol{e}_{j}}(X)\right)$, which concludes the case $r=0$.

The case $r \geq 1$ follows by reduction to the zero-dimensional case. For $1 \leq i \leq r$, let $F_{i}$ be the generic polynomial of multidegree $\boldsymbol{d}_{i}$ and $\boldsymbol{u}_{i}$ the variables corresponding to its coefficients. Set $\widetilde{k}=k\left(\boldsymbol{u}_{1}, \ldots, \boldsymbol{u}_{r}\right)$. Observe that $\operatorname{div}\left(F_{j}\right)$ intersects properly the cycle $X_{\widetilde{k}(\boldsymbol{t})} \cdot \prod_{i=1}^{j-1} \operatorname{div}\left(F_{i}\right), 1 \leq j \leq r$. Therefore, $X_{\widetilde{k}(\boldsymbol{t})} \cdot \prod_{i=1}^{r} \operatorname{div}\left(F_{i}\right)$ is a cycle of dimension 0 and, by Proposition 1.40, there exists $\lambda \in \widetilde{k}(\boldsymbol{t})^{\times}$such that

$$
\operatorname{Res}_{\boldsymbol{d}}(X)=\lambda \operatorname{Res}_{\boldsymbol{d}_{0}}\left(X_{\widetilde{k}} \cdot \prod_{i=1}^{r} \operatorname{div}\left(F_{i}\right)\right) .
$$

Since both resultants are primitive with respect to $\widetilde{k}[\boldsymbol{t}], \lambda \in \widetilde{k}^{\times}$and so these resultants have the same $\boldsymbol{t}$-degree. Analogous relations hold for $\operatorname{Res}_{\boldsymbol{e}_{j}, \boldsymbol{d}_{1}, \ldots, \boldsymbol{d}_{r}}(X), 1 \leq j \leq m$. Hence, (2.16) follows by applying the previously considered zero-dimensional case.

From Proposition 1.27, we deduce that the map

$$
\left(\mathbb{N}^{m} \backslash\{\mathbf{0}\}\right)^{r+1} \longrightarrow \mathbb{Z}, \quad \boldsymbol{d} \longmapsto \operatorname{deg}_{\boldsymbol{t}}\left(\operatorname{Res}_{\boldsymbol{d}}(X)\right)
$$

is multilinear with respect to the variables $\boldsymbol{d}_{0}, \ldots, \boldsymbol{d}_{r}$. The same holds for the map $\boldsymbol{d} \mapsto \operatorname{coeff}_{\eta \boldsymbol{\theta}^{n}}\left([X]_{k[t]} \prod_{i=0}^{r} \sum_{j=1}^{m} d_{i, j} \theta_{j}\right)$. Both maps coincide when $\boldsymbol{d}=\boldsymbol{e}(\boldsymbol{c})$ for $\boldsymbol{c} \in \mathbb{N}_{r+1}^{m}$ because

$$
\operatorname{deg}_{\boldsymbol{t}}\left(\operatorname{Res}_{\boldsymbol{e}(\boldsymbol{c})}(X)\right)=\mathrm{h}_{\boldsymbol{c}}(X)=\operatorname{coeff}_{\eta} \boldsymbol{\theta}^{n}\left(\boldsymbol{\theta}^{\boldsymbol{c}}[X]_{k[t]}\right) .
$$


Since the family $\{\boldsymbol{e}(\boldsymbol{c})\}_{\boldsymbol{c}}$ is a basis of the semigroup $\left(\mathbb{N}^{m} \backslash\{\boldsymbol{0}\}\right)^{r+1}$, both maps coincide for all $\boldsymbol{d}$. This completes the proof of the statement.

The following is an arithmetic version of Bézout's theorem for multiprojective cycles over $k(\boldsymbol{t})$.

Theorem 2.18. - Let $X \in Z_{r}\left(\underset{\mathbb{P}_{k(t)}^{\boldsymbol{n}}}{)}\right)$ and $f \in k[\boldsymbol{t}]\left[\boldsymbol{x}_{1}, \ldots, \boldsymbol{x}_{m}\right]$ a polynomial, multihomogeneous in the variables $\boldsymbol{x}$, such that $X$ and $\operatorname{div}(f)$ intersect properly.

1. If $X$ is effective, then for any $\boldsymbol{b} \in \mathbb{N}_{r}^{m}$

$$
\mathrm{h}_{\boldsymbol{b}}(X \cdot \operatorname{div}(f)) \leq \mathrm{h}(f) \operatorname{deg}_{\boldsymbol{b}}(X)+\sum_{i=1}^{m} \operatorname{deg}_{\boldsymbol{x}_{i}}(f) \mathrm{h}_{\boldsymbol{b}+\boldsymbol{e}_{i}}(X) .
$$

2. $\mathrm{h}_{\boldsymbol{c}}(X)=0$ for any $\boldsymbol{c} \in \mathbb{N}_{r+1}^{m}$ such that $c_{i}>n_{i}$ for some $i$.

3. If $X$ is effective, then $[X \cdot \operatorname{div}(f)]_{k[t]} \leq[X]_{k[t]} \cdot[\operatorname{div}(f)]_{k[t]}$.

Proof. - (1) Set $\boldsymbol{d}=\operatorname{deg}(f)$ and let $\boldsymbol{u}=\left(\boldsymbol{u}_{0}, \ldots, \boldsymbol{u}_{r-1}, \boldsymbol{u}_{r}\right)$ be the groups of variables corresponding to $(\boldsymbol{e}(\boldsymbol{b}), \boldsymbol{d})$. By Proposition 1.40, there exists $\lambda \in k(\boldsymbol{t})^{\times}$such that

$$
\operatorname{Res}_{\boldsymbol{e}(\boldsymbol{b}), \boldsymbol{d}}(X)\left(\boldsymbol{u}_{0}, \ldots, \boldsymbol{u}_{r-1}, f\right)=\lambda \operatorname{Res}_{\boldsymbol{e}(\boldsymbol{b})}(X \cdot \operatorname{div}(f))\left(\boldsymbol{u}_{0}, \ldots, \boldsymbol{u}_{r-1}\right) .
$$

Indeed, $\lambda \in k[\boldsymbol{t}] \backslash\{0\}$ because the resultant in the right-hand side is primitive. Hence,

$$
\begin{aligned}
\operatorname{deg}_{\boldsymbol{t}}\left(\operatorname{Res}_{\boldsymbol{e}(\boldsymbol{b})}(X \cdot \operatorname{div}(f))\right) & \leq \operatorname{deg}_{\boldsymbol{t}}\left(\operatorname{Res}_{\boldsymbol{e}(\boldsymbol{b}), \boldsymbol{d}}(X)\left(\boldsymbol{u}_{0}, \ldots, \boldsymbol{u}_{r-1}, f\right)\right) \\
& \leq \operatorname{deg}_{\boldsymbol{t}}(f) \operatorname{deg}_{\boldsymbol{u}_{r}}\left(\operatorname{Res}_{\boldsymbol{e}(\boldsymbol{b}), \boldsymbol{d}}(X)\right)+\operatorname{deg}_{\boldsymbol{t}}\left(\operatorname{Res}_{\boldsymbol{e}(\boldsymbol{b}), \boldsymbol{d}}(X)\right) .
\end{aligned}
$$

From the definition of the height and Proposition 1.32, we deduce that $\mathrm{h}_{\boldsymbol{b}}(X \cdot \operatorname{div}(f)) \leq$ $\mathrm{h}(f) \operatorname{deg}_{\boldsymbol{b}}(V)+\operatorname{deg}_{\boldsymbol{t}}\left(\operatorname{Res}_{\boldsymbol{e}(\boldsymbol{b}), \boldsymbol{d}}(X)\right)$. The statement follows then from (2.15).

(2) It is enough to prove the statement for a $k(\boldsymbol{t})$-variety $V$. We first consider the case when $V$ is determined by a regular sequence. We proceed by induction on its codimension. Let $f_{j}$, $1 \leq j \leq|\boldsymbol{n}|-r$, be a regular sequence of multihomogeneous polynomials. For $r \leq \ell \leq|\boldsymbol{n}|$, set

$$
Y_{\ell}=\prod_{j=1}^{|n|-\ell} \operatorname{div}\left(f_{j}\right) \in Z_{\ell}^{+}\left(\mathbb{P}_{k(t)}^{n}\right) .
$$

For $r=|\boldsymbol{n}|$, we have $Y_{|\boldsymbol{n}|}=\mathbb{P}^{\boldsymbol{n}}$ and Proposition 2.13(2) implies that $\mathrm{h}_{\boldsymbol{c}}\left(Y_{|\boldsymbol{n}|}\right)=0$ for all $\boldsymbol{c} \in \mathbb{N}_{|\boldsymbol{n}|+1}$. Suppose now that $r<|\boldsymbol{n}|$ and that the statement holds for $Y_{r+1}$. Let $\boldsymbol{c} \in \mathbb{N}_{r+1}^{m}$ such that $c_{i}>n_{i}$ for some $i$. By Proposition 2.13(1) and item (1) above,

$$
0 \leq \mathrm{h}_{\boldsymbol{c}}\left(Y_{r}\right) \leq \mathrm{h}\left(f_{|\boldsymbol{n}|-r}\right) \operatorname{deg}_{\boldsymbol{c}}\left(Y_{r+1}\right)+\sum_{j=1}^{m} \operatorname{deg}_{\boldsymbol{x}_{j}}\left(f_{|\boldsymbol{n}|-r}\right) \mathrm{h}_{\boldsymbol{c}+\boldsymbol{e}_{j}}\left(Y_{r+1}\right) .
$$

The inductive hypothesis together with the fact that $\operatorname{deg}_{c}\left(Y_{r+1}\right)=0$ imply that the righthand side of this inequality vanishes, and hence $\mathrm{h}_{\boldsymbol{c}}\left(Y_{r}\right)=0$.

For the general case, consider $|\boldsymbol{n}|-r$ generic linear combinations of a system of generators of $I(V)$. The obtained polynomials form a regular sequence and define a variety $Y_{r}$ such that $Y_{r}-V$ is effective. By the previous analysis, $0 \leq \mathrm{h}_{\boldsymbol{c}}(V) \leq \mathrm{h}_{\boldsymbol{c}}\left(Y_{r}\right)=0$, hence $\mathrm{h}_{\boldsymbol{c}}(V)=0$ as stated.

(3) This is a direct consequence of (1) and (2) together with Theorem 1.11 and Proposition 2.13(4). 
Corollary 2.19. - Let $V \subset \mathbb{P}_{k(t)}^{n}$ be a $k(\boldsymbol{t})$-variety of pure dimension $r$ and $f \in k[\boldsymbol{t}]\left[\boldsymbol{x}_{1}, \ldots, \boldsymbol{x}_{m}\right]$ a multihomogeneous polynomial. Let $W$ denote the union of the components of dimension $r-1$ of the intersection $V \cap V(f)$. Then

$$
[W]_{k[t]} \leq[V]_{k[t]} \cdot[V(f)]_{k[t]} .
$$

In particular, $\mathrm{h}_{\boldsymbol{b}}(W) \leq \mathrm{h}(f) \operatorname{deg}_{\boldsymbol{b}}(V)+\sum_{i=1}^{m} \operatorname{deg}_{\boldsymbol{x}_{i}}(f) \mathrm{h}_{\boldsymbol{b}+\boldsymbol{e}_{i}}(V)$ for all $\boldsymbol{b} \in \mathbb{N}_{r}^{m}$.

Proof. - Let $V^{\prime} \subset \mathbb{P}_{k(t)}^{n}$ be the union of the components of $V$ not contained in $|\operatorname{div}(f)|$. Then $W \subset V^{\prime} \cap V(f)$ and $V(f)$ intersects $V^{\prime}$ properly. By Theorem 2.18(3),

$$
[W]_{k(t)} \leq\left[V^{\prime} \cdot V(f)\right]_{k(t)} \leq\left[V^{\prime}\right]_{k(t} \cdot[V(f)]_{k(t)} \leq[V]_{k(t} \cdot[V(f)]_{k(t)} \cdot
$$

The last statement follows from this inequality when $\boldsymbol{b} \leq \boldsymbol{n}$ and from Theorem 2.18(2) otherwise.

Corollary 2.20. - Let $n \in \mathbb{Z}_{>0}, X \in Z_{r}^{+}\left(\mathbb{P}_{k(t)}^{n}\right)$ and $f_{j} \in k[\boldsymbol{t}]\left[x_{0}, \ldots, x_{n}\right] \backslash k[\boldsymbol{t}]$ a family of $s \leq r$ polynomials homogeneous in the variables $x_{0}, \ldots, x_{n}$ such that $X \cdot \prod_{j=1}^{i-1} \operatorname{div}\left(f_{j}\right)$ and $\operatorname{div}\left(f_{i}\right)$ intersect properly for $1 \leq i \leq s$. Then

$$
\mathrm{h}\left(X \cdot \prod_{j=1}^{s} \operatorname{div}\left(f_{j}\right)\right) \leq\left(\prod_{j=1}^{s} \operatorname{deg}_{\boldsymbol{x}}\left(f_{j}\right)\right)\left(\mathrm{h}(X)+\operatorname{deg}(X)\left(\sum_{\ell=1}^{s} \frac{\operatorname{deg}_{\boldsymbol{t}}\left(f_{\ell}\right)}{\operatorname{deg}_{\boldsymbol{x}}\left(f_{\ell}\right)}\right)\right) .
$$

Proof. - Set $Y=X \cdot \prod_{j=1}^{s} \operatorname{div}\left(f_{j}\right)$. We have

$$
[Y]_{k[t]}=\mathrm{h}(Y) \eta \theta^{n-r+s-1}+\operatorname{deg}(Y) \theta^{n-r+s},[X]_{k[t]}=\left(\mathrm{h}(X) \eta \theta^{n-r-1}+\operatorname{deg}(X) \theta^{n-r}\right) .
$$

Applying recursively Theorem 2.18(3),

$$
[Y]_{k[t]} \leq[X]_{k[t]} \cdot \prod_{j=1}^{s}\left[\operatorname{div}\left(f_{j}\right)\right]_{k[t]} .
$$

The statement follows by comparing the coefficients corresponding to the monomial $\eta \theta^{n-r+s-1}$ in the above inequality.

Next result shows that, for projective $k(t)$-cycles, the inequality in Theorem 2.18(1) is an equality in the generic case.

Proposition 2.21. - Let $X \in Z_{r}\left(\mathbb{P}_{k(t)}^{n}\right)$ with $k$ an algebraically closed field, $t$ a single variable and $n, r \geq 1$. Let $\chi \subset \mathbb{P}_{k}^{1} \times \mathbb{P}_{k}^{n}$ be the standard model of $X$ and $\ell \in k\left[x_{0}, \ldots, x_{n}\right] a$ generic linear form. Then

1. $\chi \cdot \operatorname{div}(\ell)$ is the standard model of $X \cdot \operatorname{div}(\ell)$;

2. $\operatorname{deg}(X \cdot \operatorname{div}(\ell))=\operatorname{deg}(X)$ and $\mathrm{h}(X \cdot \operatorname{div}(\ell))=\mathrm{h}(X)$.

Proof. - (1) By Lemma 2.7, it is enough to prove the claim for an irreducible $k(t)$-variety $V$ of dimension $r \geq 1$. Let $V \subset \mathbb{P}_{k}^{1} \times \mathbb{P}_{k}^{n}$ be the standard model of $V$. By Lemma 2.6(2), it suffices to prove that $V \cdot \operatorname{div}(\ell)$ is the generic fiber of $V \cdot \operatorname{div}(\ell)$ and that the support of $V \cdot \operatorname{div}(\ell)$ has no vertical components. Consider the projection $\varpi: \mathcal{V} \rightarrow \mathbb{P}_{k}^{n}$. We have

$$
1 \leq r=\operatorname{dim}(\mathcal{V})-\operatorname{dim}\left(\mathbb{P}_{k}^{1}\right) \leq \operatorname{dim}(\varpi(\mathcal{V})) \leq \operatorname{dim}(\mathcal{V})=r+1
$$

$4^{\text {e }}$ SÉRIE - TOME $46-2013$ - No 4 
If $\operatorname{dim}(\varpi(V))=1$, then $\operatorname{dim}(V)=1$ and $\varpi^{-1}(\boldsymbol{\xi})=\mathbb{P}_{k}^{1} \times\{\boldsymbol{\xi}\}$ for all $\boldsymbol{\xi} \in \varpi(\mathcal{V})$ because of the theorem of dimension of fibers. Hence, in this case,

$$
V=\mathbb{P}_{k}^{1} \times \varpi(V)
$$

Therefore, $V \cdot \operatorname{div}(\ell)=\mathbb{P}_{k}^{1} \times(\varpi(\mathcal{V}) \cdot \operatorname{div}(\ell))$ has no vertical components. Moreover, by considering the generic fiber of $\pi: \mathcal{V} \rightarrow \mathbb{P}_{k}^{1}$, it follows from Lemma 2.6(1) that $V=\varpi(\mathcal{V})_{k(t)}$. Hence, the generic fiber of $\mathcal{V} \cdot \operatorname{div}(\ell)$ over $\mathbb{P}_{k}^{1}$ coincides with $V \cdot \operatorname{div}(\ell)$, which proves the claim for $\operatorname{dim}(\varpi(\mathcal{V}))=1$.

If $\operatorname{dim}(\varpi(V)) \geq 2, V \cap V(\ell)$ is an irreducible $k$-variety of dimension $r$ by [15, Thm. 6.3(4)]. Moreover, the induced projection $\pi_{\ell}: \mathcal{V} \cdot \operatorname{div}(\ell) \rightarrow \mathbb{P}_{k}^{1}$ is surjective. Indeed, for $\tau \in \mathbb{P}^{1}(k)$, we have that $V \cap \pi^{-1}(\boldsymbol{\tau})$ is a projective variety of dimension $r \geq 1$ and so

$$
(V \cap V(\ell)) \cap \pi^{-1}(\boldsymbol{\tau})=\left(V \cap \pi^{-1}(\boldsymbol{\tau})\right) \cap V(\ell) \neq \varnothing .
$$

Hence, the projection $\pi_{\ell}$ has no vertical fibers, as it is surjective and the support of $V \cdot \operatorname{div}(\ell)$ is irreducible. By going through the ideals of definition, we can verify that its generic fiber coincides with $V \cdot \operatorname{div}(\ell)$, which completes the proof of the claim.

(2) The statement concerning the degree follows from Theorem 1.11. For the height,

$$
\mathrm{h}(X)=\operatorname{deg}_{0, r+1}(\chi)=\operatorname{deg}_{0, r}(\chi \cdot \operatorname{div}(\ell))=\mathrm{h}(X \cdot \operatorname{div}(\ell)) .
$$

The first and third equalities follow from Proposition 2.8 and the second one follows from Theorem 1.11.

We next show that mixed heights are monotonic with respect to linear projections. We keep the notation from Proposition 1.16. In particular, we consider the linear projection $\pi: \mathbb{P}_{K}^{\boldsymbol{n}} \rightarrow \mathbb{P}_{K}^{\boldsymbol{l}}$ in (1.15) and the inclusion $\jmath: A^{*}\left(\mathbb{P}^{\boldsymbol{l}} ; k[\boldsymbol{t}]\right) \hookrightarrow A^{*}\left(\mathbb{P}^{\boldsymbol{n}} ; k[\boldsymbol{t}]\right)$ defined by $\jmath(P)=\boldsymbol{\theta}^{\boldsymbol{n}-\boldsymbol{l}} P$.

Proposition 2.22. - Let $\pi: \mathbb{P}_{k(t)}^{n} \rightarrow \mathbb{P}_{k(t)}^{l}$ be the linear projection defined in (1.15) and $X \in Z_{r}^{+}\left(\underset{\mathbb{P}_{(t)}^{n}}{\boldsymbol{n}}\right)$. Then

$$
\jmath\left(\left[\pi_{*} X\right]_{k[t]}\right) \leq[X]_{k[t]} .
$$

In particular, $\mathrm{h}_{\boldsymbol{c}}\left(\pi_{*} X\right) \leq \mathrm{h}_{\boldsymbol{c}}(X)$ for all $\boldsymbol{c} \in \mathbb{N}_{r+1}^{m}$.

Proof. - The statement is equivalent to the inequalities $\operatorname{deg}_{\boldsymbol{b}}\left(\pi_{*} X\right) \leq \operatorname{deg}_{\boldsymbol{b}}(X)$ and $\mathrm{h}_{\boldsymbol{c}}\left(\pi_{*} X\right) \leq \mathrm{h}_{\boldsymbol{c}}(X)$ for all $\boldsymbol{b}, \boldsymbol{c}$. Because of Proposition 1.16, we only need to prove the latter.

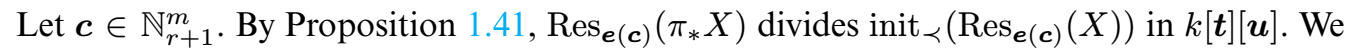
deduce that

$$
\mathrm{h}_{\boldsymbol{c}}\left(\pi_{*} X\right)=\operatorname{deg}_{\boldsymbol{t}}\left(\operatorname{Res}_{\boldsymbol{e}(\boldsymbol{c})}\left(\pi_{*} X\right)\right) \leq \operatorname{deg}_{\boldsymbol{t}}\left(\operatorname{Res}_{\boldsymbol{e}(\boldsymbol{c})}(X)\right)=\mathrm{h}_{\boldsymbol{c}}(X),
$$

which proves the statement.

The following result gives the behavior of extended Chow rings and classes with respect to products.

Proposition 2.23. - Let $m_{i} \in \mathbb{Z}_{>0}$ and $\boldsymbol{n}_{i} \in \mathbb{N}^{m_{i}}$ for $i=1,2$. Then

1. $A^{*}\left(\mathbb{P}^{\boldsymbol{n}_{1}} \times \mathbb{P}^{\boldsymbol{n}_{2}} ; k[\boldsymbol{t}]\right) \simeq A^{*}\left(\mathbb{P}^{\boldsymbol{n}_{1}} ; k[\boldsymbol{t}]\right) \otimes_{\mathbb{Z}[\eta]} A^{*}\left(\mathbb{P}^{\boldsymbol{n}_{2}} ; k[\boldsymbol{t}]\right)$. 
2. Let $X_{i} \in Z_{r_{i}}^{+}\left(\mathbb{P}_{k(t)}^{\boldsymbol{n}_{i}}\right)$ for $i=1,2$. The above isomorphism identifies $\left[X_{1} \times X_{2}\right]_{k[t]}$ with $\left[X_{1}\right]_{k[t]} \otimes\left[X_{2}\right]_{k[t]}$. In particular, for $\boldsymbol{c}_{i} \in \mathbb{N}^{m_{i}}$ such that $\left|\boldsymbol{c}_{1}\right|+\left|\boldsymbol{c}_{2}\right|=r_{1}+r_{2}+1$,

$$
\mathrm{h}_{\left(\boldsymbol{c}_{1}, \boldsymbol{c}_{2}\right)}\left(X_{1} \times X_{2}\right)=\left\{\begin{array}{cl}
\operatorname{deg}_{\boldsymbol{c}_{2}}\left(X_{2}\right) \mathrm{h}_{\boldsymbol{c}_{1}}\left(X_{1}\right) & \text { if }\left|\boldsymbol{c}_{1}\right|=r_{1}+1,\left|\boldsymbol{c}_{2}\right|=r_{2}, \\
\operatorname{deg}_{\boldsymbol{c}_{1}}\left(X_{1}\right) \mathrm{h}_{\boldsymbol{c}_{2}}\left(X_{2}\right) & \text { if }\left|\boldsymbol{c}_{1}\right|=r_{1},\left|\boldsymbol{c}_{2}\right|=r_{2}+1, \\
0 & \text { otherwise. }
\end{array}\right.
$$

Proof. - (1) is immediate from the definition of the extended Chow ring while (2) follows directly from Proposition 1.45.

Finally, we compute the class in the extended Chow ring of the ruled join of two projective varieties. Let $n_{1}, n_{2} \in \mathbb{N}$ and consider the $\mathbb{Z}$-linear map $\jmath_{i}: A^{*}\left(\mathbb{P}^{n_{i}} ; k[\boldsymbol{t}]\right) \hookrightarrow$ $A^{*}\left(\mathbb{P}^{n_{1}+n_{2}+1} ; k[\boldsymbol{t}]\right)$ defined by $\jmath_{i}\left(\theta^{l} \eta^{b}\right)=\theta^{l} \eta^{b}$ for $0 \leq l \leq n_{i}$ and $b=0,1$.

Proposition 2.24. - Let $X_{i} \in Z_{r_{i}}\left(\mathbb{P}_{k(t)}^{n_{i}}\right)$ for $i=1,2$. Then

$$
\left[X_{1} \# X_{2}\right]_{k[t]}=\jmath_{1}\left(\left[X_{1}\right]_{k[t]}\right) \cdot \jmath_{2}\left(\left[X_{2}\right]_{k[t]}\right) .
$$

In particular, $\mathrm{h}\left(X_{1} \# X_{2}\right)=\operatorname{deg}\left(X_{1}\right) \mathrm{h}\left(X_{2}\right)+\operatorname{deg}\left(X_{2}\right) \mathrm{h}\left(X_{1}\right)$.

We need some lemmas for the proof of this result. The first of them deals with 0-dimensional cycles. For $i=1,2$ and $j=0,1$, let $\boldsymbol{x}_{i}=\left\{x_{i, 0}, \ldots, x_{i, n_{i}}\right\}$ be the homogeneous coordinates of $\mathbb{P}_{k(t)}^{n_{i}}$ and $\boldsymbol{u}_{j}^{(i)}=\left\{u_{j, l}^{(i)}\right\}_{0 \leq l \leq n_{i}}$ a group of $n_{i}+1$ variables. Write $L_{j}^{(i)}$ for the general linear form in the variables $\boldsymbol{x}_{i}$.

Lemma 2.25. - Let $X_{i} \in Z_{0}\left(\mathbb{P}_{k(t)}^{n_{i}}\right)$ for $i=1,2$. Then $\mathrm{h}\left(X_{1} \# X_{2}\right)=\operatorname{deg}\left(X_{1}\right) \mathrm{h}\left(X_{2}\right)+$ $\operatorname{deg}\left(X_{2}\right) \mathrm{h}\left(X_{1}\right)$.

Proof. - For $i=1,2$, write $\operatorname{Res}_{1}\left(X_{i}\right)=\lambda_{i} \prod_{\boldsymbol{\xi}_{i}} L_{0}^{(i)}\left(\boldsymbol{\xi}_{i}\right)^{m_{\boldsymbol{\xi}_{i}}}$ with $\lambda_{i} \in \overline{k(\boldsymbol{t})}^{\times}$, $\boldsymbol{\xi}_{i} \in \mathbb{P}^{n_{i}}(\overline{k(\boldsymbol{t})})$ and $m_{\boldsymbol{\xi}_{i}} \in \mathbb{Z}$. We claim that there exists $\nu \in k(\boldsymbol{t})^{\times}$such that

$$
\operatorname{Res}_{1,1}\left(X_{1} \# X_{2}\right)=\nu \lambda_{1}^{\operatorname{deg}\left(X_{2}\right)} \lambda_{2}^{\operatorname{deg}\left(X_{1}\right)} \prod_{\boldsymbol{\xi}_{1}, \boldsymbol{\xi}_{2}}\left(L_{0}^{(1)}\left(\boldsymbol{\xi}_{1}\right) L_{1}^{(2)}\left(\boldsymbol{\xi}_{2}\right)-L_{1}^{(1)}\left(\boldsymbol{\xi}_{1}\right) L_{0}^{(2)}\left(\boldsymbol{\xi}_{2}\right)\right)^{m_{\boldsymbol{\xi}_{1}} m_{\boldsymbol{\xi}_{2}}} .
$$

Indeed, for each $\boldsymbol{\xi}_{1} \in \mathbb{P}^{n_{1}}(\overline{k(\boldsymbol{t})})$ and $\boldsymbol{\xi}_{2} \in \mathbb{P}^{n_{2}}(\overline{k(\boldsymbol{t})})$, Proposition 1.37(1) and Corollary 1.33 imply that $\operatorname{Res}_{1,1}\left(\boldsymbol{\xi}_{1} \# \boldsymbol{\xi}_{2}\right)$ is equal to $L_{0}^{(1)}\left(\boldsymbol{\xi}_{1}\right) L_{1}^{(2)}\left(\boldsymbol{\xi}_{2}\right)-L_{1}^{(1)}\left(\boldsymbol{\xi}_{1}\right) L_{0}^{(2)}\left(\boldsymbol{\xi}_{2}\right)$ up to a constant in $\overline{k(t)}^{\times}$. We deduce that there exists $\mu \in \overline{k(\boldsymbol{t})} \times$ such that

$$
\operatorname{Res}_{1,1}\left(X_{1} \# X_{2}\right)=\mu \prod_{\boldsymbol{\xi}_{1}, \boldsymbol{\xi}_{2}}\left(L_{0}^{(1)}\left(\boldsymbol{\xi}_{1}\right) L_{1}^{(2)}\left(\boldsymbol{\xi}_{2}\right)-L_{1}^{(1)}\left(\boldsymbol{\xi}_{1}\right) L_{0}^{(2)}\left(\boldsymbol{\xi}_{2}\right)\right)^{m_{\boldsymbol{\xi}_{1}} m_{\boldsymbol{\xi}_{2}}}
$$

By setting $\boldsymbol{u}_{1}^{(1)}=0$ in this equality and comparing its right-hand side with the explicit expression for $\operatorname{Res}_{1}\left(X_{i}\right)$ plus the fact that $\operatorname{Res}_{1,1}\left(X_{1} \# X_{2}\right)$ has coefficients in $k(t)$, we get that $\mu=\nu \lambda_{1}^{\operatorname{deg}\left(X_{2}\right)} \lambda_{2}^{\operatorname{deg}\left(X_{1}\right)}$ with $\nu \in k(\boldsymbol{t})^{\times}$.

Let $E$ be a sufficiently large extension of $k(\boldsymbol{t}), H \subset \mathbb{P}_{k}^{p}$ a hypersurface and $v$ a valuation of $E$ extending $\operatorname{ord}_{H}$. For $i=1,2$ and $\boldsymbol{\xi}_{i}=\left(\xi_{i, j}\right)_{j} \in E^{n_{i}+1}$ set $v\left(\boldsymbol{\xi}_{i}\right)=\min \left\{v\left(\xi_{i, j}\right)\right\}$. Observe that

$$
v\left(L_{0}^{(1)}\left(\boldsymbol{\xi}_{1}\right) L_{1}^{(2)}\left(\boldsymbol{\xi}_{2}\right)-L_{1}^{(1)}\left(\boldsymbol{\xi}_{1}\right) L_{0}^{(2)}\left(\boldsymbol{\xi}_{2}\right)\right)=v\left(\boldsymbol{\xi}_{1}\right)+v\left(\boldsymbol{\xi}_{2}\right)=v\left(L_{0}^{(1)}\left(\boldsymbol{\xi}_{1}\right)\right)+v\left(L_{0}^{(2)}\left(\boldsymbol{\xi}_{2}\right)\right) .
$$


Therefore, applying Gauss' lemma, we obtain that $\operatorname{ord}_{H}\left(\operatorname{Res}_{1,1}\left(X_{1} \# X_{2}\right)\right)$ is equal to

$$
\begin{aligned}
& \operatorname{ord}_{H}(\nu)+\operatorname{deg}\left(X_{2}\right) v\left(\lambda_{1}\right)+\operatorname{deg}\left(X_{1}\right) v\left(\lambda_{2}\right)+\sum_{\boldsymbol{\xi}_{1}, \boldsymbol{\xi}_{2}} m_{\boldsymbol{\xi}_{1}} m_{\boldsymbol{\xi}_{2}}\left(v\left(\boldsymbol{\xi}_{1}\right)+v\left(\boldsymbol{\xi}_{2}\right)\right) \\
& =\operatorname{ord}_{H}(\nu)+\operatorname{deg}\left(X_{2}\right)\left(v\left(\lambda_{1}\right)+\sum_{\boldsymbol{\xi}_{1}} m_{\boldsymbol{\xi}_{1}} v\left(\boldsymbol{\xi}_{1}\right)\right)+\operatorname{deg}\left(X_{1}\right)\left(v\left(\lambda_{2}\right)+\sum_{\boldsymbol{\xi}_{2}} m_{\boldsymbol{\xi}_{2}} v\left(\boldsymbol{\xi}_{2}\right)\right) \\
& =\operatorname{ord}_{H}(\nu)+\operatorname{deg}\left(X_{1}\right) \operatorname{ord}_{H}\left(\operatorname{Res}_{1}\left(X_{2}\right)\right)+\operatorname{deg}\left(X_{2}\right) \operatorname{ord}_{H}\left(\operatorname{Res}_{1}\left(X_{1}\right)\right) .
\end{aligned}
$$

Let $H_{\infty}$ be the hyperplane at infinity. For $H \neq H_{\infty}$,

$$
\operatorname{ord}_{H}\left(\operatorname{Res}_{1,1}\left(X_{1} \# X_{2}\right)\right)=\operatorname{ord}_{H}\left(\operatorname{Res}_{1}\left(X_{i}\right)\right)=0
$$

and the identity above implies that $\operatorname{ord}_{H}(\nu)=0$ in this case. Since this holds for all $H \neq H_{\infty}$, it follows that $\nu \in k^{\times}$. Hence, $\operatorname{ord}_{H_{\infty}}(\nu)=0$. For $H=H_{\infty}$ in the same identity, we get

$$
\operatorname{ord}_{H_{\infty}}\left(\operatorname{Res}_{1,1}\left(X_{1} \# X_{2}\right)\right)=\operatorname{deg}\left(X_{1}\right) \operatorname{ord}_{H_{\infty}}\left(\operatorname{Res}_{1}\left(X_{2}\right)\right)+\operatorname{deg}\left(X_{2}\right) \operatorname{ord}_{H_{\infty}}\left(\operatorname{Res}_{1}\left(X_{1}\right)\right) \text {. }
$$

This implies the statement.

Lemma 2.27. - Let $k$ be an algebraically closed field and $t$ a single variable. Let $V \subset \mathbb{P}_{k}^{1} \times \mathbb{P}_{k}^{n_{1}+n_{2}+1}$ be the standard model of $V_{1} \# V_{2}$, where each $V_{i} \subset \mathbb{P}_{k(t)}^{n_{i}}$ is an irreducible $k(t)$-variety and $\operatorname{dim}\left(V_{2}\right) \geq 1$. Let $\ell \in k\left[\boldsymbol{x}_{2}\right]$ be a generic linear form. Then $V \cdot \operatorname{div}(\ell)$ is the standard model of $V_{1} \#\left(V_{2} \cdot \operatorname{div}(\ell)\right)$.

Proof. - By Lemma 2.6(2), it suffices to prove that $V_{1} \#\left(V_{2} \cdot \operatorname{div}(\ell)\right)$ is the generic fiber of $V \cdot \operatorname{div}(\ell)$ and that the support of the cycle $V \cdot \operatorname{div}(\ell)$ has no vertical components.

Let $\mathscr{I}_{i, 0}=I\left(V_{i}\right) \cap k[t]\left[\boldsymbol{x}_{i}\right]$ and $\mathscr{I}_{0}=\left(I\left(V_{1}\right)+I\left(V_{2}\right)\right) \cap k[t]\left[\boldsymbol{x}_{1}, \boldsymbol{x}_{2}\right]$ be the prime ideals of $V_{i, 0}$ and of $V_{0}$ respectively, following the notation in Remark 2.5. Then $\searrow_{0} \supset \nearrow_{1,0}+\mathscr{I}_{2,0}$. In addition, there is an isomorphism of $k[t]$-algebras

$$
k[t]\left[\boldsymbol{x}_{1}, \boldsymbol{x}_{2}\right] /\left(\mathscr{I}_{1,0}+\mathscr{I}_{2,0}\right) \simeq k[t]\left[\boldsymbol{x}_{1}\right] / \mathscr{I}_{1,0} \otimes_{k[t]} k[t]\left[\boldsymbol{x}_{2}\right] / \mathscr{I}_{2,0} .
$$

Hence $\mathscr{I}_{1,0}+\mathscr{I}_{2,0}$ is a prime ideal of Krull dimension $r_{1}+r_{2}+2$. By Remark 2.5 and Lemma 2.6(1), $\mathscr{I}_{0}$ is also a prime ideal of Krull dimension $r_{1}+r_{2}+2$. Hence, $\nearrow_{0}=\nearrow_{1,0}+I_{2,0}$. This implies that $\mathscr{I}_{0}+(\ell)=I_{1,0}+I_{2,0}+(\ell)$ and so

$$
k[t]\left[\boldsymbol{x}_{1}, \boldsymbol{x}_{2}\right] /\left(\mathscr{J}_{0}+(\ell)\right) \simeq k[t]\left[\boldsymbol{x}_{1}\right] / \mathscr{I}_{1,0} \otimes_{k[t]} k[t]\left[\boldsymbol{x}_{2}\right] /\left(\mathscr{I}_{2,0}+(\ell)\right) .
$$

Let $\boldsymbol{\tau} \in \mathbb{P}^{1}(k) \backslash\{(0: 1)\}$ and write $\boldsymbol{\tau}=(1: \tau)$ with $\tau \in k$. The $k[t]$-algebra $k[t]\left[\boldsymbol{x}_{1}, \boldsymbol{x}_{2}\right] /\left(\mathscr{J}_{0}+(\ell)\right) \otimes_{k[t]} k(\tau)$ is isomorphic to

$$
\left(k[t]\left[\boldsymbol{x}_{1}\right] / \mathscr{I}_{1,0} \otimes_{k[t]} k(\tau)\right) \otimes_{k[t]}\left(k[t]\left[\boldsymbol{x}_{2}\right] /\left(\mathscr{I}_{2,0}+(\ell)\right) \otimes_{k[t]} k(\tau)\right) .
$$

The cycle $X\left(\mathscr{J}_{0}+(\ell)\right)$ coincides with $V_{0} \cdot \operatorname{div}(\ell)$ because of the definition of the intersection product. Since $\operatorname{dim}\left(V_{2}\right) \geq 1$, Proposition 2.21(1) and Lemma 2.7 imply that the cycle $X\left(\mathcal{J}_{2,0}+(\ell)\right)$ coincides with the standard model of $V_{2} \cdot \operatorname{div}(\ell)$.

We also deduce that $\operatorname{dim}\left(\pi^{-1}(\boldsymbol{\tau})\right)=r_{1}+r_{2}=\operatorname{dim}\left(\mathcal{V}_{0} \cdot \operatorname{div}(\ell)\right)-1$, and so there are no vertical components. The case when $\tau=(0: 1)$ can be treated in the same way by considering $\mathscr{I}_{\infty}$ and $\mathscr{I}_{i, \infty}$ instead of $\mathscr{I}_{0}$ and $\mathscr{I}_{i, 0}$, respectively. Finally, applying the isomorphism above to the generic point of $\mathbb{P}_{k}^{1}$, we deduce that the generic fiber of $\pi: V \cdot \operatorname{div}(\ell) \rightarrow \mathbb{P}_{k}^{1}$ coincides with $V_{1} \#\left(V_{2} \cdot \operatorname{div}(\ell)\right)$. This concludes the proof. 
Proof of Proposition 2.24. - The statement is equivalent to the equalities

$$
\operatorname{deg}\left(X_{1} \# X_{2}\right)=\operatorname{deg}\left(X_{1}\right) \operatorname{deg}\left(X_{2}\right), \quad \mathrm{h}\left(X_{1} \# X_{2}\right)=\operatorname{deg}\left(X_{1}\right) \mathrm{h}\left(X_{2}\right)+\operatorname{deg}\left(X_{2}\right) \mathrm{h}\left(X_{1}\right) .
$$

The first one is (1.20) and so we only need to prove the second. It is enough to prove this equality for irreducible $k(\boldsymbol{t})$-varieties $V_{1}, V_{2}$ over an algebraically closed field $k$.

Suppose first that the group of parameters consists in a single variable $t$. In this case, the proof will be done by induction on the dimension of the $V_{i}$ 's. Write $r_{i}=\operatorname{dim}\left(V_{i}\right)$. The case $r_{1}=r_{2}=0$ is covered by Lemma 2.25, so we assume that either $r_{1}$ or $r_{2}$ are not 0 . By symmetry, we can suppose that $r_{2} \geq 1$. Let $V_{i}$ and $V$ be the standard models of $V_{i}$ and of $V_{1} \# V_{2}$, respectively, and $\ell \in k\left[\boldsymbol{x}_{2}\right]$ a generic linear form. Then, by Proposition 2.8, Theorem 1.11, Lemma 2.27 and again Proposition 2.8,

$$
\mathrm{h}\left(V_{1} \# V_{2}\right)=\operatorname{deg}_{0, r_{1}+r_{2}+2}(\mathcal{V})=\operatorname{deg}_{0, r_{1}+r_{2}+1}(\mathcal{V} \cdot \operatorname{div}(\ell))=\mathrm{h}\left(V_{1} \#\left(V_{2} \cdot \operatorname{div}(\ell)\right)\right) .
$$

Using the inductive hypothesis and Proposition 2.21(2), we get

$$
\begin{array}{r}
\mathrm{h}\left(V_{1} \#\left(V_{2} \cdot \operatorname{div}(\ell)\right)\right)=\operatorname{deg}\left(V_{1}\right) \mathrm{h}\left(V_{2} \cdot \operatorname{div}(\ell)\right)+\operatorname{deg}\left(V_{2} \cdot \operatorname{div}(\ell)\right) \mathrm{h}\left(V_{1}\right) \\
=\operatorname{deg}\left(V_{1}\right) \mathrm{h}\left(V_{2}\right)+\operatorname{deg}\left(V_{2}\right) \mathrm{h}\left(V_{1}\right),
\end{array}
$$

which proves the statement for this case.

The case of an arbitrary number $p$ of parameters reduces to the previous one as follows. Given an irreducible subvariety $W \subset \mathbb{P}_{k(t)}^{n}$, consider the field $\widetilde{k}=k\left(\frac{t_{1}-\gamma_{1}}{w}, \ldots, \frac{t_{p}-\gamma_{p}}{w}\right)$ where $w$ is an additional variable and $\gamma_{i} \in k$ is a generic element. Observe that $\widetilde{k}(w)$ coincides with $k(\boldsymbol{t})(w)$ and so it is a transcendental extension of $k(\boldsymbol{t})$. The scalar extension $W_{\tilde{k}(w)} \subset \mathbb{P}_{\tilde{k}(w)}^{n}$ is an irreducible subvariety of the same dimension and degree as $W$. Let $\operatorname{Res}_{\mathbf{1}}\left(W_{\widetilde{k}(w)}\right)$ be the Chow form of $W_{\widetilde{k}(w)}$, primitive with respect to the base ring $\widetilde{k}[w]$. By Proposition 1.28, there exists $\lambda \in \widetilde{k}(w)^{\times}$such that

$$
\lambda \operatorname{Res}_{\mathbf{1}}\left(W_{\widetilde{k}(w)}\right)(w)=\operatorname{Res}_{\mathbf{1}}(W)(\boldsymbol{t})=\operatorname{Res}_{\mathbf{1}}(W)\left(\frac{t_{1}-\gamma_{1}}{w} w+\gamma_{1}, \ldots, \frac{t_{p}-\gamma_{p}}{w} w+\gamma_{p}\right) .
$$

Indeed, $\operatorname{Res}_{\mathbf{1}}(W)$ is a polynomial over $\widetilde{k}[w]$ and $\operatorname{Res}_{\mathbf{1}}\left(W_{\widetilde{k}(w)}\right)$ is primitive with respect to this ring. Hence, $\lambda \in \widetilde{k}[w] \backslash\{0\}$. Furthermore, let $\zeta$ be a root of $\lambda$ in the algebraic closure of $\widetilde{k}$. If $\zeta \neq 0$, setting $w=\zeta$ in the equality above we get $\operatorname{Res}_{1}(W)=0$, which is impossible. If $\zeta=0$, we get $0=\operatorname{Res}_{\mathbf{1}}(W)(\gamma)$, which is also impossible since $\gamma$ is generic. We conclude that $\lambda \in \widetilde{k}^{\times}$. In particular,

$$
\mathrm{h}(W)=\operatorname{deg}_{\boldsymbol{t}}\left(\operatorname{Res}_{\mathbf{1}}(W)\right)=\operatorname{deg}_{w}\left(\operatorname{Res}_{\mathbf{1}}\left(W_{\widetilde{k}(w)}\right)\right)=\mathrm{h}_{w}\left(W_{\widetilde{k}(w)}\right),
$$

where $\mathrm{h}_{w}$ denotes the height with respect to the base ring $\widetilde{k}[w]$. Coming back to our problem, we observe that $\left(V_{1} \# V_{2}\right)_{\tilde{k}(w)}=V_{1, \widetilde{k}(w)} \# V_{2, \widetilde{k}(w)}$, which follows readily from the definition of the ruled join. Applying (2.28) and the previously considered case when $p=1$, we conclude that $\mathrm{h}\left(V_{1} \# V_{2}\right)=\mathrm{h}_{w}\left(V_{1, \widetilde{k}(w)} \# V_{2, \widetilde{k}(w)}\right)=\operatorname{deg}\left(V_{1, \widetilde{k}(w)}\right) \mathrm{h}_{w}\left(V_{2, \widetilde{k}(w)}\right)+$ $\operatorname{deg}\left(V_{2, \widetilde{k}(w)}\right) \mathrm{h}_{w}\left(V_{1, \widetilde{k}(w)}\right)=\operatorname{deg}\left(V_{1}\right) \mathrm{h}\left(V_{2}\right)+\operatorname{deg}\left(V_{2}\right) \mathrm{h}\left(V_{1}\right)$. 


\subsection{Measures of complex polynomials}

To study cycles defined over $\mathbb{Q}$, we will use different measures for the size of a complex multivariate polynomial. We introduce them in this section and collect some of their properties.

Definition 2.29. - Let $f=\sum_{\boldsymbol{a}} \alpha_{\boldsymbol{a}} \boldsymbol{x}^{\boldsymbol{a}} \in \mathbb{C}\left[x_{1}, \ldots, x_{n}\right]$. The $\ell^{\infty}$-norm, the $\ell^{1}$-norm and the sup-norm of $f$ are respectively defined as

$$
\|f\|_{\infty}=\max _{\boldsymbol{a}}\left|\alpha_{a}\right|, \quad\|f\|_{1}=\sum_{\boldsymbol{a}}\left|\alpha_{\boldsymbol{a}}\right|, \quad\|f\|_{\sup }=\sup _{\boldsymbol{x} \in\left(S^{1}\right)^{n}}|f(\boldsymbol{x})|,
$$

where $S^{1}=\{x \in \mathbb{C}:|x|=1\}$ denotes the unit circle of $\mathbb{C}$. The Mahler measure of $f$ is defined as

$$
\mathrm{m}(f)=\int_{0}^{1} \ldots \int_{0}^{1} \log \left|f\left(\mathrm{e}^{2 \pi i u_{1}}, \ldots, \mathrm{e}^{2 \pi i u_{n}}\right)\right| d u_{1} \ldots d u_{n}=\int_{\left(S^{1}\right)^{n}} \log |f| d \mu^{n}
$$

where $\mu$ denotes the Haar measure on $S^{1}$ of total mass 1 .

We list some inequalities comparing these measures.

Lemma 2.30. - Let $f \in \mathbb{C}\left[x_{1}, \ldots, x_{n}\right]$. Then

1. $\log \|f\|_{\infty} \leq \log \|f\|_{\text {sup }} \leq \log \|f\|_{1} \leq \log \|f\|_{\infty}+\log (n+1) \operatorname{deg}(f)$;

2. $\mathrm{m}(f) \leq \log \|f\|_{\text {sup }}$;

3. $\log \|f\|_{1} \leq \mathrm{m}(f)+\log (n+1) \operatorname{deg}(f)$;

4. $\left|\mathrm{m}(f)-\log \|f\|_{\infty}\right| \leq \log (n+1) \operatorname{deg}(f)$.

Proof. - (1) Let $f=\sum_{\boldsymbol{a}} \alpha_{\boldsymbol{a}} \boldsymbol{x}^{\boldsymbol{a}}$. By Cauchy's formula, for $\boldsymbol{a} \in \operatorname{supp}(f)$,

$$
\alpha_{\boldsymbol{a}}=\int_{\left(S^{1}\right)^{n}} \frac{f(\boldsymbol{x})}{\boldsymbol{x}^{\boldsymbol{a}}} d \mu^{n} .
$$

Hence ||$f\left\|_{\infty}=\max _{\boldsymbol{a}}\left|\alpha_{\boldsymbol{a}}\right| \leq \sup _{\boldsymbol{x} \in\left(S^{1}\right)^{n}}|f(\boldsymbol{x})|=\right\| f \|_{\text {sup }}$, which gives the first inequality. The second follows easily from the definitions while the third one follows from

$$
\|f\|_{1}=\sum_{\boldsymbol{a}}\left|\alpha_{\boldsymbol{a}}\right| \leq \# \operatorname{supp}(f)\|f\|_{\infty} \leq\left(\begin{array}{c}
n+\operatorname{deg}(f) \\
n
\end{array}\right)\|f\|_{\infty} \leq(n+1)^{\operatorname{deg}(f)}\|f\|_{\infty} .
$$

(2) follows easily from the definitions, (3) follows from [27, Lem. 1.13] while (4) follows from (1), (2) and (3).

We also list some well-known properties of the Mahler measure. Recall that a weight monomial order on $\mathbb{C}[\boldsymbol{x}]$ is a partial order on the monomials of $\mathbb{C}[\boldsymbol{x}]$ defined by a vector $\boldsymbol{v} \in \mathbb{R}^{n}$ as $\boldsymbol{x}^{\boldsymbol{a}} \prec \boldsymbol{x}^{\boldsymbol{b}}$ if and only if $\langle\boldsymbol{v}, \boldsymbol{a}\rangle\langle\langle\boldsymbol{v}, \boldsymbol{b}\rangle$.

Lemma 2.31. - Let $f, g \in \mathbb{C}[\boldsymbol{x}]$. Then

1. $\mathrm{m}(f g)=\mathrm{m}(f)+\mathrm{m}(g)$.

2. $\mathrm{m}\left(f\left(\omega_{1} x_{1}, \ldots, \omega_{n} x_{n}\right)\right)=\mathrm{m}(f)$ for any $\left(\omega_{1}, \ldots, \omega_{n}\right) \in\left(S^{1}\right)^{n}$.

3. $\mathrm{m}\left(f\left(x_{1}^{\ell}, \ldots, x_{n}^{\ell}\right)\right)=\mathrm{m}(f)$ for any $\ell \geq 1$.

4. Let $\prec$ be a weight monomial order on $\mathbb{C}[\boldsymbol{x}]$. Then $\mathrm{m}\left(\operatorname{init}_{\prec}(f)\right) \leq \mathrm{m}(f)$. 
Proof. - (1), (2) These follow easily from the definition of the Mahler measure.

(3) For $\boldsymbol{u} \in \mathbb{R}^{n}$ set $\boldsymbol{w}=\left(\ell u_{1}, \ldots, \ell u_{n}\right)$ and observe that $d w_{1} \ldots d w_{n}=\ell^{n} d u_{1} \ldots d u_{n}$. Hence,

$$
\begin{array}{r}
\mathrm{m}\left(f\left(\boldsymbol{x}^{\ell}\right)\right)=\int_{[0,1]^{n}} \log \left|f\left(\mathrm{e}^{2 \pi i \ell \boldsymbol{u}}\right)\right| d u_{1} \ldots d u_{n}=\frac{1}{\ell^{n}} \int_{[0, \ell]^{n}} \log \left|f\left(\mathrm{e}^{2 \pi i \boldsymbol{w}}\right)\right| d w_{1} \ldots d w_{n} \\
=\int_{[0,1]^{n}} \log \left|f\left(\mathrm{e}^{2 \pi i \boldsymbol{w}}\right)\right| d w_{1} \ldots d w_{n}=\mathrm{m}(f(\boldsymbol{x})) .
\end{array}
$$

(4) Let $\boldsymbol{v} \in \mathbb{R}^{n}$ be a vector defining $\prec$. The exponents of the monomials of $f$ which are minimal with respect to $\prec$ are the vectors in $\operatorname{supp}(f)$ lying in the maximal face $F$ of the Newton polytope of $f$ which has $\boldsymbol{v}$ as an inner exterior normal. Hence, $\operatorname{init}_{\prec}(f)$ is the face polynomial corresponding to $F$, that is, the sum of the terms in $f$ whose exponent lies in that face. The result then follows from the fact that the Mahler measure of a polynomial is bounded below by the Mahler measure of any of its face polynomials, see for instance [36].

We collect in the following lemma some further inequalities comparing the measures of polynomials depending on groups of variables.

Lemma 2.32. - Let $f \in \mathbb{C}\left[\boldsymbol{y}_{1}, \ldots, \boldsymbol{y}_{m}\right]$ be a polynomial in $m$ groups of $n_{i}$ variables each. Then

1. $\log \|f\|_{\text {sup }} \leq \log \|f\|_{1} \leq \log \|f\|_{\infty}+\sum_{i=1}^{m} \log \left(n_{i}+1\right) \operatorname{deg}_{\boldsymbol{y}_{i}}(f)$;

2. $\left|\mathrm{m}(f)-\log \|f\|_{\infty}\right| \leq \sum_{i=1}^{m} \log \left(n_{i}+1\right) \operatorname{deg}_{\boldsymbol{y}_{i}}(f)$.

Proof. - (1) can be proved in the same way as the third inequality in Lemma 2.30(1), while (2) follows from [19, Lem. 1.1].

For multihomogeneous polynomials, we will also need the following variant of the Mahler measure, introduced and studied by Philippon in [29].

Definition 2.33. - Let $f \in \mathbb{C}\left[\boldsymbol{x}_{1}, \ldots, \boldsymbol{x}_{m}\right]$ be a multihomogeneous polynomial in $m$ groups of $n_{i}+1$ variables each. The Philippon measure of $f$ is defined as

$$
\operatorname{ph}(f)=\int_{S^{2 n_{1}+1} \times \cdots \times S^{2 n_{m}+1}} \log |f| d \mu_{1} \ldots d \mu_{m}+\sum_{i=1}^{m}\left(\sum_{j=1}^{n_{i}} \frac{1}{2 j}\right) \operatorname{deg}_{\boldsymbol{x}_{i}}(f),
$$

where $S^{2 n_{i}+1}=\left\{\boldsymbol{x} \in \mathbb{C}^{n_{i}+1}:\left|x_{0}\right|^{2}+\cdots+\left|x_{n_{i}}\right|^{2}=1\right\}$ denotes the unit sphere of $\mathbb{C}^{n_{i}+1}$ and $\mu_{i}$ the Borel measure on $S^{2 n_{i}+1}$ of total mass 1 , invariant under the action of the unitary group.

The Philippon measure is related to the Mahler measure by the inequalities

$$
0 \leq \operatorname{ph}(f)-\mathrm{m}(f) \leq \sum_{i=1}^{m} \log \left(n_{i}+1\right) \operatorname{deg}_{\boldsymbol{x}_{i}}(f),
$$

which follow from [22, Thm. 4]. In particular,

$$
\operatorname{ph}(f) \leq \log \|f\|_{\text {sup }}+\sum_{i=1}^{m} \log \left(n_{i}+1\right) \operatorname{deg}_{\boldsymbol{x}_{i}}(f) .
$$


Definition 2.36. - Let $f \in \mathbb{Z}\left[x_{1}, \ldots, x_{n}\right]$. The height of $f$ is defined as $h(f)=\log \|f\|_{\infty}$ if $f \neq 0$ and as 0 otherwise.

The following lemma estimates the behavior of the height of polynomials with respect to the arithmetic operations and composition. Its proof follows directly from the definitions.

Lemma 2.37. - Let $f_{1}, \ldots, f_{s} \in \mathbb{Z}\left[x_{1}, \ldots, x_{n}\right]$. Then

1. $\mathrm{h}\left(\sum_{i} f_{i}\right) \leq \max _{i} \mathrm{~h}\left(f_{i}\right)+\log (s)$;

2. $\mathrm{h}\left(\prod_{i} f_{i}\right) \leq \mathrm{h}\left(f_{1}\right)+\sum_{i=2}^{s} \log \left\|f_{i}\right\|_{1} \leq \sum_{i} \mathrm{~h}\left(f_{i}\right)+\log (n+1) \sum_{i=2}^{s} \operatorname{deg}\left(f_{i}\right)$.

3. Let $g \in \mathbb{C}\left[y_{1}, \ldots, y_{s}\right]$ and write $d=\max _{i} \operatorname{deg}\left(f_{i}\right)$ and $h=\max _{i} \mathrm{~h}\left(f_{i}\right)$. Then $\mathrm{h}\left(g\left(f_{1}, \ldots, f_{s}\right)\right) \leq \mathrm{h}(g)+\operatorname{deg}(g)(h+\log (s+1)+d \log (n+1))$.

\subsection{Canonical mixed heights of cycles over $\mathbb{Q}$}

The projective space $\mathbb{P}^{n}=\mathbb{P}^{n}(\overline{\mathbb{Q}})$ has a standard structure of toric variety with torus

$$
\left(\mathbb{P}^{n}\right)^{\circ}:=\mathbb{P}^{n} \backslash V\left(x_{0} \cdots x_{n}\right) \simeq\left(\overline{\mathbb{Q}}^{\times}\right)^{n} .
$$

The action of this torus on $\mathbb{P}^{n}$ writes down as $\boldsymbol{u} \cdot \boldsymbol{x}=\left(u_{0} x_{0}: \cdots: u_{n} x_{n}\right)$ for $\boldsymbol{u}=\left(u_{0}: \cdots: u_{n}\right) \in\left(\mathbb{P}^{n}\right)^{\circ}$ and $\boldsymbol{x}=\left(x_{0}: \cdots: x_{n}\right) \in \mathbb{P}^{n}$. This toric structure on $\mathbb{P}^{n}$ allows to define a notion of canonical height for its subvarieties [7]. Following David and Philippon, this height can be defined by a limit process à la Tate [10]. In precise terms, for $\ell \geq 1$, consider the $\ell$-power map

$$
[\ell]: \mathbb{P}^{n} \longrightarrow \mathbb{P}^{n}, \quad\left(x_{0}: \cdots: x_{n}\right) \longmapsto\left(x_{0}^{\ell}: \cdots: x_{n}^{\ell}\right) .
$$

Let $V \subset \mathbb{P}^{n}$ be an irreducible subvariety and let $\mathrm{h}$ denote the Fubini-Study height of projective varieties [30, 19]. The canonical (or normalized) height of $V$ can be defined as

$$
\widehat{\mathrm{h}}(V)=\operatorname{deg}(V) \lim _{\ell \rightarrow \infty} \frac{\mathrm{h}([\ell] V)}{\ell \operatorname{deg}([\ell] V)} .
$$

Both $\mathrm{h}$ and $\widehat{\mathrm{h}}$ extend to cycles by linearity. Alternatively, the canonical height can be defined using Arakelov geometry, as the height of $V$ with respect to the canonical metric on the universal line bundle $\theta(1)$, see for instance [24, 7].

We collect in the proposition below some of the basic properties of this notion. For a cycle $X$ of $\mathbb{P}^{n}$, we denote by $[\ell]_{*} X$ its direct image under the map $[\ell]$. We denote by $\mu_{\infty}$ the subgroup of $\overline{\mathbb{Q}}^{\times}$of roots of unity and by $\mu_{\ell}$ the subgroup of $\ell$-roots. A variety is called a torsion subvariety of $\mathbb{P}^{n}$ if it is the closure of the orbit of the action of a subtorus of $\left(\mathbb{P}^{n}\right)^{\circ}$ over a point with coordinates in $\{0\} \cup \mu_{\infty}$.

Proposition 2.39. - $\quad$ 1. Let $X \in Z_{r}\left(\mathbb{P}^{n}\right)$. Then $\widehat{\mathrm{h}}(X)=\lim _{\ell \rightarrow \infty} \ell^{-r-1} \mathrm{~h}\left([\ell]_{*} X\right)$.

2. Let $X \in Z_{r}\left(\mathbb{P}^{n}\right)$ and $\ell \in \mathbb{Z}_{>0}$. Then $\operatorname{deg}\left([\ell]_{*} X\right)=\ell^{r} \operatorname{deg}(X)$ and $\widehat{\mathrm{h}}\left([\ell]_{*} X\right)=$ $\ell^{r+1} \widehat{\mathrm{h}}(X)$.

3. If $X$ is an effective cycle, then $\widehat{\mathrm{h}}(X) \geq 0$.

4. If $X$ is a sum of torsion subvarieties, then $\widehat{\mathrm{h}}(X)=0$. In particular, $\widehat{\mathrm{h}}\left(\mathbb{P}^{n}\right)=0$.

5. Let $X \in Z_{r}^{+}\left(\mathbb{P}^{n}\right)$. Then $|\widehat{\mathrm{h}}(X)-\mathrm{h}(X)| \leq \frac{7}{2}(r+1) \log (n+1) \operatorname{deg}(X)$.

6. Let $\boldsymbol{\xi} \in \mathbb{P}^{n}$ be a point with rational coordinates and write $\boldsymbol{\xi}=\left(\xi_{0}: \cdots: \xi_{n}\right)$ for some coprime $\xi_{i} \in \mathbb{Z}$. Then $\widehat{\mathrm{h}}(\boldsymbol{\xi})=\log \left(\max _{i}\left|\xi_{i}\right|\right)$. 
7. Let $D \in \operatorname{Div}^{+}\left(\mathbb{P}_{\mathbb{Q}}^{n}\right)$ and $f_{D} \in \mathbb{Z}[\boldsymbol{x}]$ its primitive defining polynomial. Then $\widehat{\mathrm{h}}(D)=\mathrm{m}\left(f_{D}\right)$.

Proof. - (1)-(2) By linearity, we reduce to the case of an irreducible variety $V$. We can assume that $V \cap\left(\mathbb{P}^{n}\right)^{\circ} \neq \varnothing$, otherwise we restrict to a sufficiently small standard subspace of $\mathbb{P}^{n}$, that is, a linear subspace defined by a subset of the coordinates $x_{i}$.

For shorthand, let $[\ell]$ denote also the restriction of the $\ell$-power map to the torus $\left(\mathbb{P}^{n}\right)^{\circ}$. This is a group homomorphism with kernel $\operatorname{ker}[\ell] \simeq \mu_{\ell}^{n}$. Let $\operatorname{stab}(V)=\left\{\boldsymbol{u} \in\left(\mathbb{P}^{n}\right)^{\circ}: \boldsymbol{u} \cdot V=V\right\}$ be the stabilizer of $V$. On the one hand, by [10, Prop. 2.1(i)],

$$
\operatorname{deg}([\ell] V)=\frac{\ell^{r}}{\#(\operatorname{stab}(V) \cap \operatorname{ker}[\ell])} \operatorname{deg}(V) .
$$

On the other hand, for a generic point $\boldsymbol{x} \in V$,

$$
\operatorname{deg}\left(\left.[\ell]\right|_{V}\right)=\#\{\boldsymbol{y} \in V:[\ell] \boldsymbol{y}=[\ell] \boldsymbol{x}\}=\#\{\boldsymbol{\omega} \in \operatorname{ker}[\ell]: \boldsymbol{\omega} \boldsymbol{x} \in V\}=\#(\operatorname{stab}(V) \cap \operatorname{ker}[\ell]) .
$$

Therefore, $\operatorname{deg}\left([\ell]_{*} V\right)=\operatorname{deg}\left(\left.[\ell]\right|_{V}\right) \operatorname{deg}([\ell] V)=\ell^{r} \operatorname{deg}(V)$. Furthermore,

$$
\widehat{\mathrm{h}}(V)=\lim _{\ell \rightarrow \infty} \frac{\operatorname{deg}(V) \mathrm{h}([\ell] V)}{\ell \operatorname{deg}([\ell] V)}=\lim _{\ell \rightarrow \infty} \frac{\operatorname{deg}\left(\left.[\ell]\right|_{V}\right) \mathrm{h}([\ell] V)}{\ell^{r+1}}=\lim _{\ell \rightarrow \infty} \frac{\mathrm{h}\left([\ell]_{*} V\right)}{\ell^{r+1}} .
$$

Finally

$$
\widehat{\mathrm{h}}\left([\ell]_{*} V\right)=\lim _{j \rightarrow \infty} \frac{\mathrm{h}\left([j]_{*}[\ell]_{*} V\right)}{j^{r+1}}=\ell^{r+1} \lim _{j \rightarrow \infty} \frac{\mathrm{h}\left([j \ell]_{*} V\right)}{(\ell j)^{r+1}}=\ell^{r+1} \widehat{\mathrm{h}}(V) .
$$

(3) This is a direct consequence of the definition of $\widehat{h}$ and the analogous property for the Fubini-Study height.

(4)-(7) These follow from [10, Prop. 2.1 and display (2)].

In the sequel, we extend the notion of canonical height to the multiprojective setting and study its behavior under geometric constructions. Our approach relies on the analogous theory for the Fubini-Study mixed height developed by Rémond in [32, 33]. For simplicity, we will restrict to subvarieties of $\mathbb{P}^{\boldsymbol{n}}$ defined over $\mathbb{Q}$ or equivalently, to $\mathbb{Q}$-varieties in $\mathbb{P}_{\mathbb{Q}}^{\boldsymbol{n}}$, see Remark 1.1 . We will apply the resultant theory in $\S 1$ for the case when $A=\mathbb{Z}$. In particular, the resultant of an irreducible $\mathbb{Q}$-variety is a primitive polynomial in $\mathbb{Z}[\boldsymbol{u}]$.

Definition $2.40([33, \S 2.3])$. - Let $V \subset \mathbb{P}_{\mathbb{Q}}^{n}$ be an irreducible $\mathbb{Q}$-variety of dimension $r$, $\boldsymbol{c} \in \mathbb{N}_{r+1}^{m}$ and $\boldsymbol{e}(\boldsymbol{c})$ as defined in (1.43). The Fubini-Study (mixed) height of $V$ of index $\boldsymbol{c}$ is defined as

$$
\mathrm{h}_{\boldsymbol{c}}(V)=\operatorname{ph}\left(\operatorname{Res}_{\boldsymbol{e}(\boldsymbol{c})}(V)\right) .
$$

This definition extends by linearity to cycles in $Z_{r}\left(\mathbb{P}_{\mathbb{Q}}^{\boldsymbol{n}}\right)$.

Next, we introduce morphisms relating different multiprojective spaces.

$4^{\text {e }}$ SÉRIE - TOME $46-2013-$ No $^{\circ}$ 
Definition 2.41. - Let $\delta_{i} \in \mathbb{Z}_{>0}$ and set $N_{i}=\left(\begin{array}{c}\delta_{i}+n_{i} \\ n_{i}\end{array}\right)$. The Veronese embedding and the modified Veronese embedding of index $\delta_{i}$ are the embeddings $v_{\delta_{i}}, \widetilde{v}_{\delta_{i}}: \mathbb{P}^{n_{i}} \hookrightarrow \mathbb{P}^{N_{i}-1}$ respectively defined for $\boldsymbol{x}_{i} \in \mathbb{P}^{n_{i}}$ as

$$
v_{\boldsymbol{\delta}_{i}}\left(\boldsymbol{x}_{i}\right)=\left(\boldsymbol{x}_{i}^{\boldsymbol{a}_{i}}\right)_{\boldsymbol{a}_{i} \in \mathbb{N}_{\delta_{i}}^{n_{i}+1}}, \quad \widetilde{v}_{\boldsymbol{\delta}_{i}}\left(\boldsymbol{x}_{i}\right)=\left(\left(\begin{array}{c}
\delta_{i} \\
\boldsymbol{a}_{i}
\end{array}\right)^{1 / 2} \boldsymbol{x}_{i}^{\boldsymbol{a}_{i}}\right)_{\boldsymbol{a}_{i} \in \mathbb{N}_{\delta_{i}}^{n_{i}+1}}
$$

For $\boldsymbol{\delta}=\left(\delta_{1}, \ldots, \delta_{m}\right) \in\left(\mathbb{Z}_{>0}\right)^{m}$, we set $v_{\boldsymbol{\delta}}=v_{\delta_{1}} \times \cdots \times v_{\delta_{m}}$ and $\widetilde{v}_{\boldsymbol{\delta}}=\widetilde{v}_{\delta_{1}} \times \cdots \times \widetilde{v}_{\delta_{m}}$ for the Veronese embedding and the modified Veronese embedding of index $\boldsymbol{\delta}$, respectively. These are embeddings of $\mathbb{P}^{\boldsymbol{n}}$ into $\mathbb{P}^{\boldsymbol{N}-\mathbf{1}}$, where $\boldsymbol{N}=\left(N_{1}, \ldots, N_{m}\right)$ and $\mathbf{1}=(1, \ldots, 1)$.

Consider the Segre embedding $s: \mathbb{P}^{\boldsymbol{N}-\mathbf{1}} \hookrightarrow \mathbb{P}^{N_{1} \cdots N_{m}-1}$ defined as $s\left(\boldsymbol{y}_{1}, \ldots, \boldsymbol{y}_{m}\right)=$ $\left(y_{1, j_{1}} \cdots y_{m, j_{m}}\right)_{1 \leq j_{i} \leq N_{i}}$ for $\boldsymbol{y}_{i} \in \mathbb{P}^{N_{i}-1}$. The composed maps $s \circ v_{\boldsymbol{\delta}}, s \circ \widetilde{v}_{\boldsymbol{\delta}}: \mathbb{P}^{\boldsymbol{n}} \longrightarrow$ $\mathbb{P}^{N_{1} \cdots N_{m}-1}$ are called the Segre-Veronese embedding and the modified Segre-Veronese embedding of index $\boldsymbol{\delta}$, respectively.

Let $\boldsymbol{\delta}=\left(\delta_{1}, \ldots, \delta_{m}\right) \in\left(\mathbb{Z}_{>0}\right)^{m}$. For each $i$, consider the diagonal endomorphism $\Delta_{i}: \mathbb{P}^{N_{i}-1} \stackrel{\sim}{\rightarrow} \mathbb{P}^{N_{i}-1}$ defined as

$$
\Delta_{i}\left(\left(y_{i, \boldsymbol{a}_{i}}\right)_{\boldsymbol{a}_{i}}\right)=\left(\left(\begin{array}{c}
\delta_{i} \\
\boldsymbol{a}_{i}
\end{array}\right)^{1 / 2} y_{i, \boldsymbol{a}_{i}}\right)_{\boldsymbol{a}_{i} \in \mathbb{N}_{\delta_{i}}^{n_{i}+1}} .
$$

Consider also the diagonal endomorphism $\Delta: \mathbb{P}^{N_{1} \cdots N_{m}-1} \stackrel{\sim}{\rightarrow} \mathbb{P}^{N_{1} \cdots N_{m}-1}$ defined as

$$
\Delta\left(\left(y_{\boldsymbol{a}}\right)_{\boldsymbol{a}}\right)=\left(\left(\begin{array}{c}
\delta_{1} \\
\boldsymbol{a}_{1}
\end{array}\right)^{1 / 2} \cdots\left(\begin{array}{c}
\delta_{m} \\
\boldsymbol{a}_{m}
\end{array}\right)^{1 / 2} y_{\boldsymbol{a}}\right)_{\boldsymbol{a} \in \mathbb{N}_{\delta}^{n+1}} .
$$

These linear maps allow to write the modified Veronese and the modified Segre-Veronese embeddings in terms of the Veronese and Segre-Veronese embeddings as

$$
\widetilde{v}_{\delta_{i}}=\Delta_{i} \circ v_{\delta_{i}}, \quad s \circ \widetilde{v}_{\boldsymbol{\delta}}=\Delta \circ s \circ v_{\boldsymbol{\delta}} .
$$

The degree and the Fubini-Study height of the direct image of a cycle $X \in Z_{r}\left(\mathbb{P}_{\mathbb{Q}}^{\boldsymbol{n}}\right)$ under the Segre-Veronese and the modified Segre-Veronese embeddings decompose in terms of mixed degrees and Fubini-Study mixed heights of $X$.

Proposition 2.44. - Let $X \in Z_{r}\left(\mathbb{P}_{\mathbb{Q}}^{\boldsymbol{n}}\right)$ and $\boldsymbol{\delta} \in\left(\mathbb{Z}_{>0}\right)^{m}$. Then

$$
\operatorname{deg}\left(\left(s \circ v_{\boldsymbol{\delta}}\right)_{*} X\right)=\sum_{\boldsymbol{b} \in \mathbb{N}_{r}^{m}}\left(\begin{array}{l}
r \\
\boldsymbol{b}
\end{array}\right) \operatorname{deg}_{\boldsymbol{b}}(X) \boldsymbol{\delta}^{\boldsymbol{b}}, \mathrm{h}\left(\left(s \circ \widetilde{v}_{\boldsymbol{\delta}}\right)_{*} X\right)=\sum_{\boldsymbol{c} \in \mathbb{N}_{r+1}^{m}}\left(\begin{array}{c}
r+1 \\
\boldsymbol{c}
\end{array}\right) \mathrm{h}_{\boldsymbol{c}}(X) \boldsymbol{\delta}^{\boldsymbol{c}}
$$

Proof. - This follows from [33, p. 103].

Let $\ell \in \mathbb{Z}_{>0}$. The $\ell$-power map of $\mathbb{P}^{n}$ is defined as

$$
[\ell]: \mathbb{P}^{\boldsymbol{n}} \longrightarrow \mathbb{P}^{\boldsymbol{n}}, \quad \boldsymbol{x}=\left(x_{i, j}\right)_{i, j} \longmapsto \boldsymbol{x}^{\ell}=\left(x_{i, j}^{\ell}\right)_{i, j} .
$$


Proposition-Definition 2.45. - Let $X \in Z_{r}\left(\mathbb{P}_{\mathbb{Q}}^{\boldsymbol{n}}\right)$ and $\boldsymbol{c} \in \mathbb{N}_{r+1}^{m}$. Then the sequence $\left(\ell^{-r-1} \mathrm{~h}_{c}\left([\ell]_{*} X\right)\right)_{\ell \geq 1}$ converges for $\ell \rightarrow \infty$. The limit

$$
\widehat{\mathrm{h}}_{\boldsymbol{c}}(X):=\lim _{\ell \rightarrow \infty} \ell^{-r-1} \mathrm{~h}_{\boldsymbol{c}}\left([\ell]_{*} X\right)
$$

is called the canonical (mixed) height of $X$ of index $\boldsymbol{c}$. For any $\boldsymbol{\delta} \in\left(\mathbb{Z}_{>0}\right)^{m}$ it holds

$$
\widehat{\mathrm{h}}\left(\left(s \circ v_{\boldsymbol{\delta}}\right)_{*} X\right)=\sum_{\boldsymbol{c} \in \mathbb{N}_{r+1}^{m}}\left(\begin{array}{c}
r+1 \\
\boldsymbol{c}
\end{array}\right) \widehat{\mathrm{h}}_{\boldsymbol{c}}(X) \boldsymbol{\delta}^{\boldsymbol{c}} .
$$

In particular, for a projective cycle $X \in Z_{r}\left(\mathbb{P}_{\mathbb{Q}}^{n}\right)$ we have that $\widehat{\mathrm{h}}_{r+1}(X)=\widehat{\mathrm{h}}(X)$.

Proof. - Proposition 2.44 applied to the cycle $[\ell]_{*} X$ implies that

$$
\mathrm{h}\left(\left(s \circ \widetilde{v}_{\boldsymbol{\delta}}\right)_{*}[\ell]_{*} X\right)=\sum_{\boldsymbol{c} \in \mathbb{N}_{r+1}^{m}}\left(\begin{array}{c}
r+1 \\
\boldsymbol{c}
\end{array}\right) \mathrm{h}_{\boldsymbol{c}}\left([\ell]_{*} X\right) \boldsymbol{\delta}^{\boldsymbol{c}} .
$$

Let $I \subset\left(\mathbb{Z}_{>0}\right)^{m}$ be a subset of cardinality $\#\left(\mathbb{N}_{r+1}^{m}\right)$ such that the square matrix $\left(\left(\begin{array}{c}r+1 \\ c\end{array}\right) \boldsymbol{\delta}^{c}\right)_{\boldsymbol{c} \in \mathbb{N}_{r+1}^{m}, \boldsymbol{\delta} \in I}$ is of maximal rank. Inverting this matrix, we can write the mixed heights in the formula above in terms of heights of projective cycles as

$$
\mathrm{h}_{\boldsymbol{c}}\left([\ell]_{*} X\right)=\sum_{\boldsymbol{\delta} \in I} \nu_{\boldsymbol{c}, \boldsymbol{\delta}} \mathrm{h}\left(\left(s \circ \widetilde{v}_{\boldsymbol{\delta}}\right)_{*}[\ell]_{*} X\right)
$$

with $\nu_{c, \delta} \in \mathbb{Q}$ not depending on $\ell$. Observe that

$$
\left(s \circ \widetilde{v}_{\boldsymbol{\delta}} \circ[\ell]\right)_{*} X=\Delta_{*}\left(s \circ v_{\boldsymbol{\delta}} \circ[\ell]\right)_{*} X,
$$

where $\Delta$ denotes the linear map in (2.42). By [19, Lem. 2.7] applied to the projective cycle $\left(s \circ v_{\delta} \circ[\ell]\right)_{*} X$, the map $\Delta$ and its inverse, there exists $\kappa(m, r, \delta) \geq 0$ such that the quantity $\left|\mathrm{h}\left(\left(s \circ \widetilde{v}_{\boldsymbol{\delta}}\right)_{*}[\ell]_{*} X\right)-\mathrm{h}\left(\left(s \circ v_{\boldsymbol{\delta}}\right)_{*}[\ell]_{*} X\right)\right|$ is bounded above by

$$
\kappa(m, r, \boldsymbol{\delta}) \log \left(\prod_{i=1}^{m}\left(\begin{array}{c}
\delta_{i}+n_{i} \\
n_{i}
\end{array}\right)\right) \operatorname{deg}\left(\left(s \circ v_{\boldsymbol{\delta}}\right)_{*}[\ell]_{*} X\right) .
$$

We have that $[\ell]$ commutes with $s \circ v_{\boldsymbol{\delta}}$. By Proposition 2.39(2),

$$
\operatorname{deg}\left(\left(s \circ v_{\boldsymbol{\delta}}\right)_{*}[\ell]_{*} X\right)=\operatorname{deg}\left([\ell]_{*}\left(s \circ v_{\boldsymbol{\delta}}\right)_{*} X\right)=\ell^{r} \operatorname{deg}\left(\left(s \circ v_{\boldsymbol{\delta}}\right)_{*} X\right) .
$$

We deduce $\mathrm{h}\left(\left(s \circ \widetilde{v}_{\boldsymbol{\delta}}\right)_{*}[\ell]_{*} X\right)=\mathrm{h}\left([\ell]_{*}\left(s \circ v_{\boldsymbol{\delta}}\right)_{*} X\right)+O\left(\ell^{r}\right)$. Therefore, for each $\boldsymbol{\delta} \in I$,

$$
\lim _{\ell \rightarrow \infty} \ell^{-r-1} \mathrm{~h}\left(\left(s \circ \widetilde{v}_{\delta}\right)_{*}[\ell]_{*} X\right)=\lim _{\ell \rightarrow \infty} \ell^{-r-1} \mathrm{~h}\left([\ell]_{*}\left(s \circ v_{\delta}\right)_{*} X\right)=\widehat{\mathrm{h}}\left(\left(s \circ v_{\boldsymbol{\delta}}\right)_{*} X\right) .
$$

This proves that the sequence $\left(\ell^{-r-1} \mathrm{~h}_{c}\left([\ell]_{*} X\right)\right)_{\ell \geq 1}$ converges for $\ell \rightarrow \infty$, since it is a linear combination of convergent sequences as shown in (2.48). The Formula (2.46) follows from (2.47) by passing to the limit for $\ell \rightarrow \infty$. The last statement is this formula applied to $X \in Z_{r}\left(\mathbb{P}_{\mathbb{Q}}^{n}\right)$ and $\delta=1$.

Remark 2.49. - The definition of canonical mixed heights in [31, Formula (1.3)] is different from the one presented here. Nevertheless, both notions coincide as they both satisfy (2.46). These mixed heights can be alternatively defined using Arakelov geometry, as explained at the end of [31, §I], and they correspond to the canonical mixed heights induced by the toric structure of $\mathbb{P}_{\mathbb{Q}}^{n}$, see [7].

$4{ }^{\text {e }}$ SÉRIE - TOME $46-2013$ - No 4 
Definition 2.50. - Let $\eta$ be an indeterminate. The extended Chow ring of $\mathbb{P}_{\mathbb{Q}}^{n}$ is the graded ring

$$
A^{*}\left(\mathbb{P}_{\mathbb{Q}}^{\boldsymbol{n}} ; \mathbb{Z}\right)=A^{*}\left(\mathbb{P}_{\mathbb{Q}}^{\boldsymbol{n}}\right) \otimes_{\mathbb{Z}} \mathbb{R}[\eta] /\left(\eta^{2}\right) \simeq \mathbb{R}\left[\eta, \theta_{1}, \ldots, \theta_{m}\right] /\left(\eta^{2}, \theta_{1}^{n_{1}+1}, \ldots, \theta_{m}^{n_{m}+1}\right),
$$

where $\theta_{i}$ denotes the class in $A^{*}\left(\mathbb{P}_{\mathbb{Q}}^{\boldsymbol{n}}\right)$ of the inverse image of a hyperplane of $\mathbb{P}_{\mathbb{Q}}^{n_{i}}$ under the projection $\mathbb{P}_{\mathbb{Q}}^{n} \rightarrow \mathbb{P}_{\mathbb{Q}}^{n_{i}}$. For short, we alternatively denote this ring as $A^{*}\left(\mathbb{P}^{n} ; \mathbb{Z}\right)$. To a cycle $X \in Z_{r}\left(\mathbb{P}_{\mathbb{Q}}^{\boldsymbol{n}}\right)$ we associate an element of this ring, defined as

$$
[X]_{\mathbb{Z}}=\sum_{\boldsymbol{c} \in \mathbb{N}_{r+1}^{m}, \boldsymbol{c} \leq \boldsymbol{n}} \widehat{\mathrm{h}}_{\boldsymbol{c}}(X) \eta \theta_{1}^{n_{1}-c_{1}} \cdots \theta_{m}^{n_{m}-c_{m}}+\sum_{\boldsymbol{b} \in \mathbb{N}_{r}^{m}, \boldsymbol{b} \leq \boldsymbol{n}} \operatorname{deg}_{\boldsymbol{b}}(X) \theta_{1}^{n_{1}-b_{1}} \cdots \theta_{m}^{n_{m}-b_{m}}
$$

It is a homogeneous element of degree $|\boldsymbol{n}|-r$.

There is an inclusion $\imath: A^{*}\left(\mathbb{P}_{\mathbb{Q}}^{\boldsymbol{n}}\right) \hookrightarrow A^{*}\left(\mathbb{P}_{\mathbb{Q}}^{\boldsymbol{n}} ; \mathbb{Z}\right)$ which satisfies $[X]_{\mathbb{Z}} \equiv \imath([X])(\bmod \eta)$. In particular, the class of a cycle in the Chow ring is determined by its class in the extended Chow ring.

For $X \in Z_{r}\left(\mathbb{P}_{\mathbb{Q}}^{\boldsymbol{n}}\right)$, Theorem 2.58(2) shows that $\widehat{\mathrm{h}}_{\boldsymbol{c}}(X)=0$ for every $\boldsymbol{c}$ such that $c_{i}>n_{i}$ for some $i$. Hence $[X]_{\mathbb{Z}}$ contains the information of all mixed degrees and heights, since $\left\{\boldsymbol{\theta}^{\boldsymbol{a}}, \eta \boldsymbol{\theta}^{\boldsymbol{a}}\right\}_{\boldsymbol{a} \leq \boldsymbol{n}}$ is a basis of $A^{*}\left(\mathbb{P}^{\boldsymbol{n}} ; \mathbb{Z}\right)$.

Next proposition extends the first properties of the canonical height in Proposition 2.39 to the multiprojective setting. The space $\mathbb{P}^{n}$ is a toric variety with torus

$$
\left(\mathbb{P}^{\boldsymbol{n}}\right)^{\circ}=\prod_{i=1}^{m}\left(\mathbb{P}^{n_{i}}\right)^{\circ} \simeq\left(\overline{\mathbb{Q}}^{\times}\right)^{|\boldsymbol{n}|} .
$$

A variety $V$ is called a torsion subvariety of $\mathbb{P}^{n}$ if it is the closure of the orbit of the action of a subtorus of $\left(\mathbb{P}^{\boldsymbol{n}}\right)^{\circ}$ over a point with coordinates in $\{0\} \cup \mu_{\infty}$.

Proposition 2.51. - $\quad$ 1. Let $X \in Z_{r}\left(\mathbb{P}_{\mathbb{Q}}^{\boldsymbol{n}}\right)$ and $\ell \geq 1$. Then $\operatorname{deg}_{\boldsymbol{b}}\left([\ell]_{*} X\right)=\ell^{r} \operatorname{deg}_{\boldsymbol{b}}(X)$ and $\widehat{\mathrm{h}}_{\boldsymbol{c}}\left([\ell]_{*} X\right)=\ell^{r+1} \widehat{\mathrm{h}}_{\boldsymbol{c}}(X)$ for all $\boldsymbol{b} \in \mathbb{N}_{r}^{m}$ and $\boldsymbol{c} \in \mathbb{N}_{r+1}^{m}$.

2. Let $X \in Z_{r}^{+}\left(\mathbb{P}_{\mathbb{Q}}^{\boldsymbol{n}}\right)$. Then $[X]_{\mathbb{Z}} \geq 0$. In particular, $\widehat{\mathrm{h}}_{\boldsymbol{c}}(X) \geq 0$ for all $\boldsymbol{c} \in \mathbb{N}_{r+1}^{m}$.

3. If $X$ is a linear combination of torsion subvarieties, then $[X]_{\mathbb{Z}}=\imath([X])$ or equivalently, $\widehat{\mathrm{h}}_{\boldsymbol{c}}(X)=0$ for all $\boldsymbol{c} \in \mathbb{N}_{r+1}^{m}$. In particular, $\left[\mathbb{P}_{\mathbb{Q}}^{\boldsymbol{n}}\right]_{\mathbb{Z}}=1$.

4. Let $X \in Z_{r}^{+}\left(\mathbb{P}_{\mathbb{Q}}^{\boldsymbol{n}}\right)$. Then there exists $\kappa(r, m) \geq 0$ such that for all $\boldsymbol{c} \in \mathbb{N}_{r+1}^{m}$,

$$
\left|\widehat{\mathrm{h}}_{\boldsymbol{c}}(X)-\mathrm{h}_{\boldsymbol{c}}(X)\right| \leq \kappa(r, m) \log (|\boldsymbol{n}|+1) \sum_{\boldsymbol{b} \in \mathbb{N}_{r}^{m}} \operatorname{deg}_{\boldsymbol{b}}(X)
$$

Proof. - (1) Recall that $[\ell]$ commutes with $s \circ v_{\boldsymbol{\delta}}$. Hence, Propositions 2.44 and 2.39(2) imply that

$$
\begin{aligned}
\ell^{r} \sum_{\boldsymbol{b} \in \mathbb{N}_{r}^{m}}\left(\begin{array}{l}
r \\
\boldsymbol{b}
\end{array}\right) \operatorname{deg}_{\boldsymbol{b}}(X) \boldsymbol{\delta}^{\boldsymbol{b}}=\ell^{r} \operatorname{deg} & \left(\left(s \circ v_{\boldsymbol{\delta}}\right)_{*} X\right) \\
& =\operatorname{deg}\left(\left(s \circ v_{\boldsymbol{\delta}}\right)_{*}[\ell]_{*} X\right)=\sum_{\boldsymbol{b} \in \mathbb{N}_{r}^{m}}\left(\begin{array}{l}
r \\
\boldsymbol{b}
\end{array}\right) \operatorname{deg}_{\boldsymbol{b}}\left([\ell]_{*} X\right) \boldsymbol{\delta}^{\boldsymbol{b}} .
\end{aligned}
$$

Since this holds for all $\boldsymbol{\delta} \in\left(\mathbb{Z}_{>0}\right)^{m}$, we deduce that $\operatorname{deg}_{\boldsymbol{b}}\left([\ell]_{*} X\right)=\ell^{r} \operatorname{deg}_{\boldsymbol{b}}(X)$. The statement for the height follows analogously by using (2.46) and Proposition 2.39(2).

(2) The non-negativity of the canonical mixed heights is a consequence of the nonnegativity of the Fubini-Study mixed heights. The latter follows from the estimates in (2.34) 
and the non-negativity of the Mahler measure of a polynomial with integer coefficients. Hence, $\widehat{\mathrm{h}}_{\boldsymbol{c}}(X) \geq 0$ for all $c \in \mathbb{N}_{r+1}^{m}$. The rest of the statement follows from this together with Proposition 1.10(1).

(3) It suffices to prove this statement for a torsion subvariety $V$ of $\mathbb{P}^{n}$. Given $\boldsymbol{\delta} \in\left(\mathbb{Z}_{>0}\right)^{m}$, Proposition 2.39(4) implies that $\widehat{\mathrm{h}}\left(\left(s \circ v_{\delta}\right)_{*} X\right)=0$ since the image of $V$ under a SegreVeronese embedding is a torsion subvariety of a projective space. Therefore, the right-hand side of (2.46) is equal to 0 for all $\boldsymbol{\delta}$, which implies that all its coefficients are 0 . Hence all canonical mixed heights of $V$ are 0 , as stated.

(4) By (2.46) and Proposition 2.44, for each $\boldsymbol{\delta} \in\left(\mathbb{Z}_{>0}\right)^{m}$,

$$
\widehat{\mathrm{h}}\left(\left(s \circ v_{\boldsymbol{\delta}}\right)_{*} X\right)-\mathrm{h}\left(\left(s \circ \widetilde{v}_{\boldsymbol{\delta}}\right)_{*} X\right)=\sum_{\boldsymbol{c} \in \mathbb{N}_{r+1}^{m}}\left(\begin{array}{c}
r+1 \\
\boldsymbol{c}
\end{array}\right)\left(\widehat{\mathrm{h}}_{\boldsymbol{c}}(X)-\mathrm{h}_{\boldsymbol{c}}(X)\right) \boldsymbol{\delta}^{\boldsymbol{c}} .
$$

As in the proof of Proposition-Definition 2.45, we pick a subset $I \subset\left(\mathbb{Z}_{>0}\right)^{m}$ of cardinality $\#\left(\mathbb{N}_{r+1}^{m}\right)$ such that the square matrix $M_{I}:=\left(\left(\begin{array}{c}r+1 \\ c\end{array}\right) \boldsymbol{\delta}^{c}\right)_{c \in \mathbb{N}_{r+1}^{m}, \boldsymbol{\delta} \in I}$ is of maximal rank. Inverting this matrix, we obtain for each $c$

$$
\widehat{\mathrm{h}}_{\boldsymbol{c}}(X)-\mathrm{h}_{\boldsymbol{c}}(X)=\sum_{\boldsymbol{\delta} \in I} \nu_{\boldsymbol{c}, \boldsymbol{\delta}}\left(\widehat{\mathrm{h}}\left(\left(s \circ v_{\boldsymbol{\delta}}\right)_{*} X\right)-\mathrm{h}\left(\left(s \circ \widetilde{v}_{\boldsymbol{\delta}}\right)_{*} X\right)\right)
$$

with $\nu_{\boldsymbol{c}, \boldsymbol{\delta}} \in \mathbb{Q}$. Using (2.43), [19, Lem. 2.7], Proposition 2.44 and the inequality $\log \left(\prod_{i=1}^{m}\left(\begin{array}{c}\delta_{i}+n_{i} \\ n_{i}\end{array}\right)\right) \leq|\boldsymbol{\delta}| \log (|\boldsymbol{n}|+1)$, we get that there exists $\kappa_{1}(r, m, \boldsymbol{\delta}) \geq 0$ such that

$$
\left|\mathrm{h}\left(\left(s \circ \widetilde{v}_{\boldsymbol{\delta}}\right)_{*} X\right)-\mathrm{h}\left(\left(s \circ v_{\boldsymbol{\delta}}\right)_{*} X\right)\right| \leq \kappa_{1}(r, m, \boldsymbol{\delta}) \log (|\boldsymbol{n}|+1) \sum_{\boldsymbol{b} \in \mathbb{N}_{r}^{m}} \operatorname{deg}_{\boldsymbol{b}}(X) .
$$

By Propositions 2.39(5) and 2.44, there exists $\kappa_{2}(r, m, \boldsymbol{\delta}) \geq 0$ such that

$$
\left|\widehat{\mathrm{h}}\left(\left(s \circ v_{\boldsymbol{\delta}}\right)_{*} X\right)-\mathrm{h}\left(\left(s \circ v_{\boldsymbol{\delta}}\right)_{*} X\right)\right| \leq \kappa_{2}(r, m, \boldsymbol{\delta}) \log (|\boldsymbol{n}|+1) \sum_{\boldsymbol{b} \in \mathbb{N}_{r}^{m}} \operatorname{deg}_{\boldsymbol{b}}(X) .
$$

Therefore, setting $\kappa_{3}(r, m, \boldsymbol{\delta})=\kappa_{1}(r, m, \boldsymbol{\delta})+\kappa_{2}(r, m, \boldsymbol{\delta})$, we get

$$
\left|\widehat{\mathrm{h}}\left(\left(s \circ v_{\boldsymbol{\delta}}\right)_{*} X\right)-\mathrm{h}\left(\left(s \circ \widetilde{v}_{\boldsymbol{\delta}}\right)_{*} X\right)\right| \leq \kappa_{3}(r, m, \boldsymbol{\delta}) \log (|\boldsymbol{n}|+1) \sum_{\boldsymbol{b} \in \mathbb{N}_{r}^{m}} \operatorname{deg}_{\boldsymbol{b}}(X) .
$$

Observe that the matrix $M_{I}$ does not depend on $\boldsymbol{n}$. Using (2.52), we deduce that there exists $\kappa(r, m)$ such that

$$
\left|\widehat{\mathrm{h}}_{\boldsymbol{c}}(X)-\mathrm{h}_{\boldsymbol{c}}(X)\right| \leq \kappa(r, m) \log (|\boldsymbol{n}|+1) \sum_{\boldsymbol{b} \in \mathbb{N}_{r}^{m}} \operatorname{deg}_{\boldsymbol{b}}(X)
$$

for all $c \in \mathbb{N}_{r+1}^{m}$, as stated.

The following proposition describes the mixed heights and classes of points and divisors, extending Proposition 2.39(6)-(7) to the multiprojective setting.

Proposition 2.53. - $\quad$ 1. Let $\boldsymbol{\xi}=\left(\boldsymbol{\xi}_{1}, \ldots, \boldsymbol{\xi}_{m}\right) \in \mathbb{P}^{\boldsymbol{n}}$ be a point with coordinates in $\mathbb{Q}$ and for each $1 \leq i \leq m$ write $\boldsymbol{\xi}_{i}=\left(\xi_{i, j}\right)_{j}$ for coprime $\xi_{i, j} \in \mathbb{Z}$. Then

$$
[\boldsymbol{\xi}]_{\mathbb{Z}}=\sum_{i=1}^{m} \widehat{\mathrm{h}}\left(\boldsymbol{\xi}_{i}\right) \eta \boldsymbol{\theta}^{\boldsymbol{n}-\boldsymbol{e}_{i}}+\boldsymbol{\theta}^{\boldsymbol{n}}
$$


In particular, $\widehat{\mathrm{h}}_{\boldsymbol{e}_{i}}(\boldsymbol{\xi})=\widehat{\mathrm{h}}\left(\boldsymbol{\xi}_{i}\right)$.

2. Let $D \in \operatorname{Div}^{+}\left(\mathbb{P}_{\mathbb{Q}}^{n}\right)$ and $f_{D} \in \mathbb{Z}[\boldsymbol{x}]$ its primitive defining polynomial. Then

$$
[D]_{\mathbb{Z}}=\mathrm{m}\left(f_{D}\right) \eta+\sum_{i=1}^{m} \operatorname{deg}_{\boldsymbol{x}_{i}}\left(f_{D}\right) \theta_{i}
$$

In particular, $\widehat{\mathrm{h}}_{\boldsymbol{n}}(D)=\mathrm{m}\left(f_{D}\right)$.

We need the following lemma.

Lemma 2.54. - Let $H$ be an irreducible $\mathbb{Q}$-hypersurface of $\mathbb{P}_{\mathbb{Q}}^{\boldsymbol{n}}$ which is not a standard hyperplane and $\ell \geq 1$. Then

$$
f_{[\ell] *[\ell]_{*} H}=\prod_{\boldsymbol{\omega} \in \operatorname{ker}[\ell]} f_{H}(\boldsymbol{\omega} \cdot \boldsymbol{x}) .
$$

Proof. - Let $\operatorname{stab}(H)=\left\{\boldsymbol{u} \in\left(\mathbb{P}^{n}\right)^{\circ}: \boldsymbol{u} \cdot H=H\right\}$ be the stabilizer of $V$. Then

$$
[\ell]^{-1}[\ell] H=\bigcup_{\omega} \boldsymbol{\omega} \cdot H
$$

the union being over a set of representatives of $\operatorname{ker}[\ell] /(\operatorname{stab}(H) \cap \operatorname{ker}[\ell])$. As in the proof of Proposition 2.39(1)-(2), we can show that $\operatorname{deg}\left(\left.[\ell]\right|_{H}\right)=\#(\operatorname{stab}(H) \cap \operatorname{ker}[\ell])$. Hence,

$$
[\ell]^{*}[\ell]_{*} H=\operatorname{deg}\left(\left.[\ell]\right|_{H}\right)[\ell]^{-1}[\ell] H=\sum_{\boldsymbol{\omega} \in \operatorname{ker}[\ell]} \boldsymbol{\omega} \cdot H .
$$

This implies that the primitive polynomial defining this divisor satisfies

$$
f_{[\ell] *[\ell]_{*} H}=\lambda \prod_{\boldsymbol{\omega} \in \operatorname{ker}[\ell]} f_{H}(\boldsymbol{\omega} \cdot \boldsymbol{x})
$$

with $\lambda \in \mathbb{Q}^{\times}$. It only remains to prove that $\lambda= \pm 1$. Let $K$ be the $\ell$-th cyclotomic field, so that $f_{H}(\boldsymbol{\omega} \cdot \boldsymbol{x}) \in K[\boldsymbol{x}]$ for all $\boldsymbol{\omega} \in \operatorname{ker}[\ell]$. Let $p \in \mathbb{Z}$ be a prime number and $v$ a valuation of $K$ extending $\operatorname{ord}_{p}$. By Gauss Lemma,

$$
\operatorname{ord}_{p}\left(\prod_{\boldsymbol{\omega}} f_{H}(\boldsymbol{\omega} \cdot \boldsymbol{x})\right)=\sum_{\boldsymbol{\omega}} v\left(f_{H}(\boldsymbol{\omega} \cdot \boldsymbol{x})\right)=0
$$

since $f_{H}$ is primitive and $v(\boldsymbol{\omega})=0$ for all $\boldsymbol{\omega} \in \operatorname{ker}[\ell]$. This implies that $\operatorname{ord}_{p}(\lambda)=0$ for all $p$ and $\lambda= \pm 1$ as desired.

Proof of Proposition 2.53. - (1) It is enough to prove that $\widehat{\mathrm{h}}_{\boldsymbol{e}_{i}}(\boldsymbol{\xi})=\widehat{\mathrm{h}}\left(\boldsymbol{\xi}_{i}\right)$. We have that $[\ell]_{*} \boldsymbol{\xi}=\boldsymbol{\xi}^{\ell}$, hence $\operatorname{Res}_{\boldsymbol{e}_{i}}\left([\ell]_{*} \boldsymbol{\xi}\right)=\lambda_{i} L_{i}\left(\boldsymbol{\xi}_{i}^{\ell}\right)$, where $L_{i}$ is the general linear form of multidegree $\boldsymbol{e}_{i}$ and $\lambda_{i} \in \mathbb{Q}^{\times}$, thanks to Corollary 1.38. Since $\operatorname{Res}_{\boldsymbol{e}_{i}}(\boldsymbol{\xi})$ and $L_{i}\left(\boldsymbol{\xi}_{i}\right)$ are primitive polynomials, we deduce that $\lambda_{i}= \pm 1$. Applying Definition 2.40, the estimates in (2.34) and Lemma 2.30(4), we obtain

$$
\ell^{-1} \mathrm{~h}_{\boldsymbol{e}_{i}}\left([\ell]_{*} \boldsymbol{\xi}\right)=\ell^{-1} \operatorname{ph}\left(L_{i}\left(\boldsymbol{\xi}_{i}^{\ell}\right)\right)=\log \left(\max _{j}\left|\xi_{i, j}\right|\right)+O\left(\ell^{-1}\right) .
$$

The statement follows by letting $\ell \rightarrow \infty$.

(2) By Proposition 1.10(4),

$$
[D]_{\mathbb{Z}}=\widehat{\mathrm{h}}_{\boldsymbol{n}}(D) \eta+[D]=\widehat{\mathrm{h}}_{\boldsymbol{n}}(D) \eta+\sum_{i=1}^{m} \operatorname{deg}_{\boldsymbol{x}_{i}}\left(f_{D}\right) \theta_{i} .
$$


Thus, we only have to prove that $\widehat{\mathrm{h}}_{\boldsymbol{n}}(D)=\mathrm{m}\left(f_{D}\right)$. We reduce without loss of generality to the case of an irreducible hypersurface $H$. If $H$ is a standard hyperplane of $\mathbb{P}_{\mathbb{Q}}^{n}$, then $f_{H}=x_{i, j}$ for some $i, j$. Hence $\widehat{\mathrm{h}}_{\boldsymbol{n}}(H)=0=\mathrm{m}\left(x_{i, j}\right)$, and the statement is true in this case.

We can then suppose that $H$ is an irreducible $\mathbb{Q}$-hypersurface which is not a standard hyperplane. Let $\ell \geq 1$. By definition, $f_{[\ell]^{*}[\ell]_{*} H}=f_{[\ell]_{*} H}\left(\boldsymbol{x}^{\ell}\right)$. Applying successively Lemma 2.31(3), Lemma 2.54 and Lemma 2.31(2), we get

$$
\mathrm{m}\left(f_{[\ell]_{*} H}\right)=\mathrm{m}\left(f_{[\ell]_{*} H}\left(\boldsymbol{x}^{\ell}\right)\right)=\sum_{\omega \in \operatorname{ker}[\ell]} \mathrm{m}\left(f_{H}(\omega \cdot \boldsymbol{x})\right)=\ell^{|\boldsymbol{n}|} \mathrm{m}\left(f_{H}\right) .
$$

By [33, Thm. 3.4], the estimates in (2.34) and Proposition 2.51(1),

$$
\mathrm{h}_{\boldsymbol{n}}\left([\ell]_{*} H\right)=\operatorname{ph}\left(f_{[\ell]_{*} H}\right)+\sum_{i=1}^{m}\left(\sum_{j=1}^{n_{i}-1} \sum_{l=1}^{j} \frac{1}{2 l}\right) \operatorname{deg}_{\boldsymbol{n}-\boldsymbol{e}_{i}}\left([\ell]_{*} H\right)=\mathrm{m}\left(f_{[\ell]_{*} H}\right)+O\left(\ell^{|\boldsymbol{n}|-1}\right) .
$$

Therefore, $\ell^{-|\boldsymbol{n}|} \mathrm{h}_{\boldsymbol{n}}\left([\ell]_{*} H\right)=\mathrm{m}\left(f_{H}\right)+O\left(\ell^{-1}\right)$. We conclude that $\widehat{\mathrm{h}}_{\boldsymbol{n}}(H)=\mathrm{m}\left(f_{H}\right)$ by letting $\ell \rightarrow \infty$.

The following result gives the behavior of mixed degrees and heights with respect to Veronese embeddings.

Proposition 2.55. - For $\boldsymbol{\delta} \in\left(\mathbb{Z}_{>0}\right)^{m}$ let $v_{\boldsymbol{\delta}}$ be the Veronese embedding of index $\boldsymbol{\delta}$, $X \in Z_{r}\left(\mathbb{P}_{\mathbb{Q}}^{\boldsymbol{n}}\right), \boldsymbol{b} \in \mathbb{N}_{r}^{m}$ and $\boldsymbol{c} \in \mathbb{N}_{r+1}^{m}$. Then

$$
\operatorname{deg}_{\boldsymbol{b}}\left(\left(v_{\boldsymbol{\delta}}\right)_{*} X\right)=\boldsymbol{\delta}^{\boldsymbol{b}} \operatorname{deg}_{\boldsymbol{b}}(X), \quad \widehat{\mathrm{h}}_{\boldsymbol{c}}\left(\left(v_{\boldsymbol{\delta}}\right)_{*} X\right)=\boldsymbol{\delta}^{\boldsymbol{c}} \widehat{\mathrm{h}}_{\boldsymbol{c}}(X) .
$$

Proof. - It is enough to prove the statement for an irreducible $\mathbb{Q}$-variety $V$. Set $N_{i}=\left(\begin{array}{c}\delta_{i}+n_{i} \\ n_{i}\end{array}\right)$ for $1 \leq i \leq m, \boldsymbol{N}=\left(N_{1}, \ldots, N_{m}\right)$ and $\mathbf{1}=(1, \ldots, 1) \in \mathbb{N}^{m}$. The embedding $v_{\boldsymbol{\delta}}$ induces an isomorphism of multigraded algebras

$$
\mathbb{Q}\left[\mathbb{P}^{\boldsymbol{N}-\mathbf{1}}\right] / I\left(v_{\boldsymbol{\delta}}(V)\right)=\bigoplus_{\boldsymbol{d} \in \mathbb{N}^{m}}\left(\mathbb{Q}\left[\mathbb{P}^{\boldsymbol{N}-\mathbf{1}}\right] / I\left(v_{\boldsymbol{\delta}}(V)\right)\right)_{\boldsymbol{d}} \simeq \bigoplus_{\boldsymbol{d} \in \mathbb{N}^{m}}\left(\mathbb{Q}\left[\mathbb{P}^{\boldsymbol{n}}\right] / I(V)\right)_{\left(d_{1} \delta_{1}, \ldots, d_{m} \delta_{m}\right)} .
$$

Hence, the Hilbert-Samuel functions of $V$ and of $v_{\boldsymbol{\delta}}(V)$ satisfy $H_{v_{\boldsymbol{\delta}}(V)}\left(d_{1}, \ldots, d_{m}\right)=$ $H_{V}\left(\delta_{1} d_{1}, \ldots, \delta_{m} d_{m}\right)$ for all $\boldsymbol{d}$. Comparing the coefficient of the monomial $\boldsymbol{d}^{\boldsymbol{b}}$ in the corresponding Hilbert polynomials and using that $v_{\delta}$ is a map of degree 1 , it follows that $\operatorname{deg}_{\boldsymbol{b}}\left(\left(v_{\boldsymbol{\delta}}\right)_{*} V\right)=\operatorname{deg}_{\boldsymbol{b}}\left(v_{\boldsymbol{\delta}}(V)\right)=\boldsymbol{\delta}^{\boldsymbol{b}} \operatorname{deg}_{\boldsymbol{b}}(V)$.

Concerning the height, consider the embeddings

$$
s \circ v_{\boldsymbol{d} \boldsymbol{\delta}}: \mathbb{P}^{\boldsymbol{n}} \longleftrightarrow \mathbb{P}^{N_{1}^{\prime} \cdots N_{m}^{\prime}-1}, \quad s \circ v_{\boldsymbol{d}} \circ v_{\boldsymbol{\delta}}: \mathbb{P}^{\boldsymbol{n}} \longleftrightarrow \mathbb{P}^{M_{1} \cdots M_{m}-1},
$$

where $N_{i}^{\prime}=\left(\begin{array}{c}d_{i} \delta_{i}+n_{i} \\ n_{i}\end{array}\right)$ and $M_{i}=\left(\begin{array}{c}d_{i}+N_{i}-1 \\ N_{i}-1\end{array}\right)$. For $\boldsymbol{x}_{i} \in \mathbb{P}^{n_{i}}$,

$$
v_{d_{i} \delta_{i}}\left(\boldsymbol{x}_{i}\right)=\left(\boldsymbol{x}_{i}^{\boldsymbol{a}_{i}}\right)_{\left|\boldsymbol{a}_{i}\right|=d_{i} \delta_{i}}, \quad v_{d_{i}} \circ v_{\delta_{i}}\left(\boldsymbol{x}_{i}\right)=\left(\left(\left(\boldsymbol{x}_{i}^{\boldsymbol{b}_{i}}\right)_{\left|\boldsymbol{b}_{i}\right|=\delta_{i}}\right)^{\boldsymbol{c}_{i}}\right)_{\left|\boldsymbol{c}_{i}\right|=d_{i}} .
$$

Observe that the monomials appearing in the image of both maps are the same. This implies that there are linear maps $A_{i}: \mathbb{P}^{M_{i}-1} \rightarrow \mathbb{P}^{N_{i}^{\prime}-1}$ and $B_{i}: \mathbb{P}^{N_{i}^{\prime}-1} \rightarrow \mathbb{P}^{M_{i}-1}$ such that $A_{i} \circ v_{d_{i}} \circ v_{\delta_{i}}=v_{d_{i} \delta_{i}}$ and $v_{d_{i}} \circ v_{\delta_{i}}=B_{i} \circ v_{d_{i} \delta_{i}}$. In turn, this implies that there exist linear maps $A: \mathbb{P}^{M_{1} \cdots M_{m}-1} \rightarrow \mathbb{P}^{N_{1}^{\prime} \cdots N_{m}^{\prime}-1}$ and $B: \mathbb{P}^{N_{1}^{\prime} \cdots N_{m}^{\prime}-1} \rightarrow \mathbb{P}^{M_{1} \cdots M_{m}-1}$ such that

$$
A \circ s \circ v_{\boldsymbol{d}} \circ v_{\boldsymbol{\delta}}=s \circ v_{\boldsymbol{d} \boldsymbol{\delta}}, \quad s \circ v_{\boldsymbol{d}} \circ v_{\boldsymbol{\delta}}=B \circ s \circ v_{\boldsymbol{d} \boldsymbol{\delta}} .
$$

4 e SÉRIE - TOME $46-2013-$ No $^{\circ}$ 
Let $\ell \geq 1$. We apply [19, Lem. 2.7] to compare the Fubini-Study height of the image of the cycle $[\ell]_{*} V$ under the maps $s \circ v_{\boldsymbol{d} \boldsymbol{\delta}}$ and $s \circ v_{\boldsymbol{d}} \circ v_{\boldsymbol{\delta}}$. Using also Propositions 2.44 and 2.51(1), we obtain that there exists $\kappa(\boldsymbol{n}, \boldsymbol{d}, \boldsymbol{\delta}) \geq 0$ such that

$$
\left|\mathrm{h}\left(s \circ v_{\boldsymbol{d} \boldsymbol{\delta}}\left([\ell]_{*} V\right)\right)-\mathrm{h}\left(s \circ v_{\boldsymbol{d}} \circ v_{\boldsymbol{\delta}}\left([\ell]_{*} V\right)\right)\right| \leq \kappa(\boldsymbol{n}, \boldsymbol{d}, \boldsymbol{\delta}) \sum_{\boldsymbol{b}} \operatorname{deg}_{\boldsymbol{b}}\left([\ell]_{*} V\right)=O\left(\ell^{r}\right) .
$$

The map $[\ell]$ commutes with $v_{\boldsymbol{\delta}}, v_{\boldsymbol{d}}, v_{\boldsymbol{d} \delta}$ and $s$. Hence, $s \circ v_{\boldsymbol{d} \delta}\left([\ell]_{*} V\right)=[\ell]_{*} s \circ v_{\boldsymbol{d} \delta}(V)$ and $s \circ v_{\boldsymbol{d}} \circ v_{\boldsymbol{\delta}}\left([\ell]_{*} V\right)=[\ell]_{*} s \circ v_{\boldsymbol{d}} \circ v_{\boldsymbol{\delta}}(V)$. From (2.56), we deduce that $\mathrm{h}\left([\ell]_{*} s \circ v_{\boldsymbol{d}} \circ v_{\boldsymbol{\delta}}(V)\right)=$ $\mathrm{h}\left([\ell]_{*} s \circ v_{\boldsymbol{d} \delta}(V)\right)+O\left(\ell^{r}\right)$. Therefore,

$$
\begin{aligned}
\widehat{\mathrm{h}}\left(s \circ v_{\boldsymbol{d}} \circ v_{\boldsymbol{\delta}}(V)\right)=\lim _{\ell \rightarrow \infty} \ell^{-r-1} h\left([\ell]_{*} s \circ v_{\boldsymbol{d}} \circ v_{\boldsymbol{\delta}}(V)\right) \\
\quad=\lim _{\ell \rightarrow \infty} \ell^{-r-1} \mathrm{~h}\left([\ell]_{*} s \circ v_{\boldsymbol{d} \boldsymbol{\delta}}(V)\right)=\widehat{\mathrm{h}}\left(s \circ v_{\boldsymbol{d} \boldsymbol{\delta}}(V)\right) .
\end{aligned}
$$

Hence, Proposition 2.44 implies that

$$
\begin{aligned}
\sum_{\boldsymbol{c}}\left(\begin{array}{c}
r+1 \\
\boldsymbol{c}
\end{array}\right) \widehat{\mathrm{h}}_{\boldsymbol{c}}\left(v_{\boldsymbol{\delta}}(V)\right) \boldsymbol{d}^{\boldsymbol{c}}=\widehat{\mathrm{h}}\left(s \circ v_{\boldsymbol{d}} \circ v_{\boldsymbol{\delta}}(V)\right) & \\
& =\widehat{\mathrm{h}}\left(s \circ v_{\boldsymbol{d} \boldsymbol{\delta}}(V)\right)=\sum_{\boldsymbol{c}}\left(\begin{array}{c}
r+1 \\
\boldsymbol{c}
\end{array}\right) \widehat{\mathrm{h}}_{\boldsymbol{c}}(V)(\boldsymbol{d} \boldsymbol{\delta})^{\boldsymbol{c}} .
\end{aligned}
$$

As this holds for all $\boldsymbol{d} \in \mathbb{N}^{m}$, it follows that $\widehat{\mathrm{h}}_{\boldsymbol{c}}\left(v_{\boldsymbol{\delta}}(V)\right)=\boldsymbol{\delta}^{\boldsymbol{c}} \widehat{\mathrm{h}}_{\boldsymbol{c}}(V)$, as stated.

The following result is the arithmetic analogue of Bézout's theorem for multiprojective $\mathbb{Q}$-cycles. It contains Theorem 3 in the introduction. Given a multihomogeneous polynomial $f \in \mathbb{Z}\left[\boldsymbol{x}_{1}, \ldots, \boldsymbol{x}_{m}\right]$, we consider the element in the extended Chow ring

$$
[f]_{\text {sup }}=\log \|f\|_{\text {sup }} \eta+\sum_{i=1}^{m} \operatorname{deg}_{\boldsymbol{x}_{i}}(f) \theta_{i} \in A^{*}\left(\mathbb{P}^{n} ; \mathbb{Z}\right) .
$$

Observe that $[\operatorname{div}(f)]_{\mathbb{Z}} \leq[f]_{\text {sup }}$.

Theorem 2.58. - Let $X \in Z_{r}\left(\mathbb{P}_{\mathbb{Q}}^{\boldsymbol{n}}\right)$ and $f \in \mathbb{Z}\left[\boldsymbol{x}_{1}, \ldots, \boldsymbol{x}_{m}\right]$ a multihomogeneous polynomial such that $X$ and $\operatorname{div}(f)$ intersect properly.

1. If $X$ is effective, then for any $\boldsymbol{b} \in \mathbb{N}_{r}^{m}$,

$$
\widehat{\mathrm{h}}_{\boldsymbol{b}}(X \cdot \operatorname{div}(f)) \leq \sum_{i=1}^{m} \operatorname{deg}_{\boldsymbol{x}_{i}}(f) \widehat{\mathrm{h}}_{\boldsymbol{b}+\boldsymbol{e}_{i}}(X)+\log \|f\|_{\text {sup }} \operatorname{deg}_{\boldsymbol{b}}(X) .
$$

2. $\widehat{\mathrm{h}}_{\boldsymbol{c}}(X)=0$ for any $\boldsymbol{c} \in \mathbb{N}_{r+1}^{m}$ such that $c_{i}>n_{i}$ for some $i$.

3. If $X$ is effective, then $[X \cdot \operatorname{div}(f)]_{\mathbb{Z}} \leq[X]_{\mathbb{Z}} \cdot[f]_{\text {sup }}$.

Proof. - (1) We reduce without loss of generality to the case of an irreducible $\mathbb{Q}$-variety $V$. Let $\ell \geq 1$ and set $\ell=(\ell, \ldots, \ell) \in\left(\mathbb{Z}_{>0}\right)^{m}$. Consider the Veronese embedding $v_{\ell}: \mathbb{P}^{\boldsymbol{n}} \rightarrow \mathbb{P}^{\boldsymbol{N}-\mathbf{1}}$ where $\boldsymbol{N}=\left(\left(\begin{array}{c}\ell+n_{i} \\ n_{i}\end{array}\right), \ldots,\left(\begin{array}{c}\ell+n_{i} \\ n_{i}\end{array}\right)\right), \mathbf{1}=(1, \ldots, 1) \in \mathbb{N}^{m}$. Set $\boldsymbol{d}=\left(d_{1}, \ldots, d_{m}\right)=\operatorname{deg}(f)$. We have that $f^{\ell} \in \mathbb{Q}\left[\mathbb{P}^{\boldsymbol{n}}\right]_{\ell \boldsymbol{d}}$ and so there is a unique polynomial $F_{\ell} \in \mathbb{Q}\left[\mathbb{P}^{\boldsymbol{N}-\mathbf{1}}\right]_{\boldsymbol{d}}$ such that $v_{\ell}^{*} \operatorname{div}\left(F_{\ell}\right)=\operatorname{div}\left(f^{\ell}\right)=\ell \operatorname{div}(f)$. Then, the projection Formula (1.6) implies that

$$
\left(v_{\ell}\right)_{*} V \cdot \operatorname{div}\left(F_{\ell}\right)=\ell\left(v_{\ell}\right)_{*}(V \cdot \operatorname{div}(f)) .
$$


Set $\|F\|_{v_{\ell}(V)}=\sup _{\boldsymbol{\xi} \in v_{\ell}(V)} \frac{\left|F_{\ell}(\boldsymbol{\xi})\right|}{\| \boldsymbol{\xi}_{1}||^{d_{1} \ldots}|| \boldsymbol{\xi}_{m}||^{d_{m}}}$, where $\|\cdot\|$ is the Euclidean norm. We apply the arithmetic Bézout's theorem for the Fubini-Study mixed height ([33, Thm. 3.4 and Cor. 3.6]) to the variety $Z_{\ell}:=v_{\ell}(V)=\left(v_{\ell}\right)_{*} V$ and the $\operatorname{divisor} \operatorname{div}\left(F_{\ell}\right)$ and we obtain

$$
\mathrm{h}_{\boldsymbol{b}}\left(Z_{\ell} \cdot \operatorname{div}\left(F_{\ell}\right)\right) \leq \sum_{i=1}^{m} d_{i} \mathrm{~h}_{\boldsymbol{b}+\boldsymbol{e}_{i}}\left(Z_{\ell}\right)+\operatorname{deg}_{\boldsymbol{b}}\left(Z_{\ell}\right) \log \left(\left\|F_{\ell}\right\|_{v_{\ell}(V)}\right) .
$$

Applying Proposition 2.51(4) to $Z_{\ell}$ and to $Z_{\ell} \cdot \operatorname{div}\left(F_{\ell}\right)$ together with the multiprojective Bézout's Theorem 1.11, it follows that there exists $\kappa(r, m, \boldsymbol{d}) \geq 0$ such that

$$
\begin{aligned}
\widehat{\mathrm{h}}_{\boldsymbol{b}}\left(Z_{\ell} \cdot \operatorname{div}\left(F_{\ell}\right)\right) \leq \sum_{i=1}^{m} d_{i} \widehat{\mathrm{h}}_{\boldsymbol{b}+\boldsymbol{e}_{i}}\left(Z_{\ell}\right) & +\operatorname{deg}_{\boldsymbol{b}}\left(Z_{\ell}\right) \log \left(\left\|F_{\ell}\right\|_{v_{\ell}(V)}\right) \\
& +\kappa(r, m, \boldsymbol{d}) \log (|\boldsymbol{N}-\mathbf{1}|+1) \sum_{\boldsymbol{b}} \operatorname{deg}_{\boldsymbol{b}}\left(Z_{\ell}\right) .
\end{aligned}
$$

By Proposition 2.55, $\operatorname{deg}_{\boldsymbol{b}}\left(Z_{\ell}\right)=\ell^{r} \operatorname{deg}_{\boldsymbol{b}}(V)$ and $\widehat{\mathrm{h}}_{\boldsymbol{b}+\boldsymbol{e}_{i}}\left(Z_{\ell}\right)=\ell^{r+1} \widehat{\mathrm{h}}_{\boldsymbol{b}+\boldsymbol{e}_{i}}(V)$. The same result together with (2.59) also implies that

$$
\widehat{\mathrm{h}}_{\boldsymbol{b}}\left(Z_{\ell} \cdot \operatorname{div}\left(F_{\ell}\right)\right)=\widehat{\mathrm{h}}_{\boldsymbol{b}}\left(\ell v_{\ell, *}(V \cdot \operatorname{div}(f))\right)=\ell^{r+1} \widehat{\mathrm{h}}_{\boldsymbol{b}}(V \cdot \operatorname{div}(f)) .
$$

We have that

$$
\begin{aligned}
\left\|F_{\ell}\right\|_{v_{\ell}(V)}=\sup _{\boldsymbol{x} \in V} \frac{\left|f^{\ell}(\boldsymbol{x})\right|}{\left\|v_{\ell}\left(\boldsymbol{x}_{1}\right)\right\|^{d_{1}} \cdots} & \left\|v_{\ell}\left(\boldsymbol{x}_{m}\right)\right\|^{d_{m}} \\
& \leq \sup _{\boldsymbol{x} \in V} \frac{\left|f^{\ell}(\boldsymbol{x})\right|}{\prod_{i=1}^{m} \max _{j}\left|x_{i, j}\right|^{\ell d_{i}}} \leq \sup _{\boldsymbol{x} \in \mathbb{D}^{n+1}}\left|f^{\ell}(\boldsymbol{x})\right|,
\end{aligned}
$$

where $\mathbb{D}=\{z \in \mathbb{C}:|z| \leq 1\}$ is the unit disk. Hence, $\log \left(\left\|F_{\ell}\right\|_{v_{\ell}(V)}\right) \leq \ell \log \|f\|_{\text {sup }}$ because of the maximum modulus principle applied to $f$. Besides, $\log (|\boldsymbol{N}-\mathbf{1}|+1)=O(\log (\ell))$ and we deduce from (2.60) that

$$
\widehat{\mathrm{h}}_{\boldsymbol{b}}(V \cdot \operatorname{div}(f)) \leq \sum_{i=1}^{m} d_{i} \widehat{\mathrm{h}}_{\boldsymbol{b}+\boldsymbol{e}_{i}}(V)+\operatorname{deg}_{\boldsymbol{b}}(V) \log \|f\|_{\text {sup }}+O\left(\frac{\log (\ell)}{\ell}\right) .
$$

The result follows by letting $\ell \rightarrow \infty$.

(2) This follows by adapting the proof of Theorem 2.18(2) to this setting without major changes.

(3) This is an immediate consequence of (1) and (2), together with Theorem 1.11 and Proposition 1.10(4).

The following results can be proved by adapting the arguments in the proofs of corollaries 2.19 and 2.20 without major changes.

Corollary 2.61. - Let $V \subset \mathbb{P}_{\mathbb{Q}}^{n}$ be a $\mathbb{Q}$-variety of pure dimension $r$ and $f \in \mathbb{Z}\left[\boldsymbol{x}_{1}, \ldots, \boldsymbol{x}_{m}\right]$ a multihomogeneous polynomial. Let $W$ denote the union of the components of dimension $r-1$ of the intersection $V \cap V(f)$. Then

$$
[W]_{\mathbb{Z}} \leq[V]_{\mathbb{Z}} \cdot[V(f)]_{\mathrm{sup}} .
$$

In particular, $\widehat{\mathrm{h}}_{\boldsymbol{b}}(W) \leq \sum_{i=1}^{m} \operatorname{deg}_{\boldsymbol{x}_{i}}(f) \widehat{\mathrm{h}}_{\boldsymbol{b}+\boldsymbol{e}_{i}}(V)+\log \|f\|_{\text {sup }} \operatorname{deg}_{\boldsymbol{b}}(V)$ for all $\boldsymbol{b} \in \mathbb{N}_{r}^{m}$. 
Corollary 2.62. - Let $n \in \mathbb{Z}_{>0}, X \in Z_{r}^{+}\left(\mathbb{P}_{\mathbb{Q}}^{n}\right)$ and $f_{j} \in \mathbb{Z}\left[x_{0}, \ldots, x_{n}\right] \backslash \mathbb{Z}$ be $s \leq r$ homogeneous polynomials such that $X \cdot \prod_{j=1}^{i-1} \operatorname{div}\left(f_{j}\right)$ and $\operatorname{div}\left(f_{i}\right)$ intersect properly for all $1 \leq i \leq s$. Then

$$
\widehat{\mathrm{h}}\left(X \cdot \prod_{j=1}^{s} \operatorname{div}\left(f_{j}\right)\right) \leq\left(\prod_{i=1}^{s} \operatorname{deg}\left(f_{i}\right)\right)\left(\widehat{\mathrm{h}}(X)+\operatorname{deg}(X)\left(\sum_{\ell=1}^{s} \frac{\log \left\|f_{\ell}\right\|_{\text {sup }}}{\operatorname{deg}\left(f_{\ell}\right)}\right)\right) .
$$

REMARK 2.63. - Theorem 2.58 is one of the main reasons for considering canonical mixed heights instead of others. For instance, the fact that the canonical mixed heights of index $c$ such that $c_{i}>n_{i}$ for some $i$ are zero is quite convenient in the applications. Observe that the analogue of this statement for the Fubini-Study mixed heights does not hold: for instance, $\mathrm{h}_{n+1}\left(\mathbb{P}^{n}\right)$ equals the $n$-th Stoll number, which is not zero. This fact implies that the class in $A^{*}\left(\mathbb{P}_{\mathbb{Q}}^{n} ; \mathbb{Z}\right)$ of a cycle $X$ contains the information about all canonical mixed heights of $X$. This information is necessary if one wants to express the arithmetic Bézout's inequality in Theorem 2.58(1) in terms of elements in the extended Chow ring.

We next show that canonical mixed heights are monotonic with respect to linear projections. We keep the notation from Proposition 1.16 and consider also the injective $\mathbb{R}$-linear map $\jmath: A^{*}\left(\mathbb{P}^{l} ; \mathbb{Z}\right) \hookrightarrow A^{*}\left(\mathbb{P}^{\boldsymbol{n}} ; \mathbb{Z}\right)$ defined by $\jmath(P)=\boldsymbol{\theta}^{\boldsymbol{n}-\boldsymbol{l}} P$.

Proposition 2.64. - Let $\pi: \mathbb{P}_{\mathbb{Q}}^{n} \rightarrow \mathbb{P}_{\mathbb{Q}}^{l}$ be the standard projection defined in (1.15) and $X \in Z_{r}^{+}\left(\mathbb{P}_{\mathbb{Q}}^{n}\right)$. Then

In particular, $\widehat{\mathrm{h}}_{\boldsymbol{c}}\left(\pi_{*} X\right) \leq \widehat{\mathrm{h}}_{\boldsymbol{c}}(X)$ for all $\boldsymbol{c} \in \mathbb{N}_{r+1}^{m}$.

$$
\jmath\left(\left[\pi_{*} X\right]_{\mathbb{Z}}\right) \leq[X]_{\mathbb{Z}} .
$$

Proof. - The statement is equivalent to $\operatorname{deg}_{\boldsymbol{b}}\left(\pi_{*} X\right) \leq \operatorname{deg}_{\boldsymbol{b}}(X)$ and $\widehat{\mathrm{h}}_{\boldsymbol{c}}\left(\pi_{*} X\right) \leq \widehat{\mathrm{h}}_{\boldsymbol{c}}(X)$ for all $\boldsymbol{b}, \boldsymbol{c}$. Because of Proposition 1.16, we only need to prove the latter inequality.

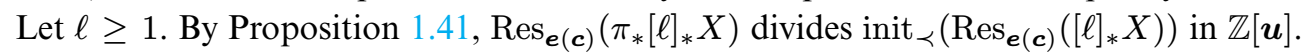
Using the fact that the Mahler measure of a polynomial with integers coefficients is $\geq 0$ and Lemma 2.31(4), we deduce that

$$
\mathrm{m}\left(\operatorname{Res}_{\boldsymbol{e}(\boldsymbol{c})}\left(\pi_{*}[\ell]_{*} X\right)\right) \leq \mathrm{m}\left(\operatorname{init}_{\prec}\left(\operatorname{Res}_{\boldsymbol{e}(\boldsymbol{c})}\left([\ell]_{*} X\right)\right)\right) \leq \mathrm{m}\left(\operatorname{Res}_{\boldsymbol{e}(\boldsymbol{c})}\left([\ell]_{*} X\right)\right) .
$$

From the estimates in (2.34) and Proposition 2.51(1), it follows that

$$
\begin{aligned}
& \left.\mathrm{h}_{\boldsymbol{c}}\left(\pi_{*}[\ell]_{*} X\right)\right)=\operatorname{ph}\left(\operatorname{Res}_{\boldsymbol{e}(\boldsymbol{c})}\left(\pi_{*}[\ell]_{*} X\right)\right)=\mathrm{m}\left(\operatorname{Res}_{\boldsymbol{e}(\boldsymbol{c})}\left(\pi_{*}[\ell]_{*} X\right)\right)+O\left(\ell^{r}\right), \\
& \left.\mathrm{h}_{\boldsymbol{c}}\left([\ell]_{*} X\right)\right)=\operatorname{ph}\left(\operatorname{Res}_{\boldsymbol{e}(\boldsymbol{c})}\left([\ell]_{*} X\right)\right)=\mathrm{m}\left(\operatorname{Res}_{\boldsymbol{e}(\boldsymbol{c})}\left([\ell]_{*} X\right)\right)+O\left(\ell^{r}\right) .
\end{aligned}
$$

Since $\pi$ commutes with $[\ell]$,

$$
\widehat{\mathrm{h}}_{\boldsymbol{c}}\left(\pi_{*}(X)\right)=\lim _{\ell \rightarrow \infty} \frac{\mathrm{h}_{\boldsymbol{c}}\left([\ell]_{*} \pi_{*}(X)\right)}{\ell^{r+1}}=\lim _{\ell \rightarrow \infty} \frac{\mathrm{h}_{\boldsymbol{c}}\left(\pi_{*}[\ell]_{*} X\right)}{\ell^{r+1}} \leq \lim _{\ell \rightarrow \infty} \frac{\mathrm{h}_{\boldsymbol{c}}\left([\ell]_{*} X\right)}{\ell^{r+1}}=\widehat{\mathrm{h}}_{\boldsymbol{c}}(X),
$$

which completes the proof.

EXAMPLE 2.65. - We consider the problem in Example 1.13 of computing the pairs (eigenvalue, eigenvector) for the case of a matrix $M$ with entries in $\mathbb{Z}$. We use the notations therein and furthermore we set $Z=\prod_{i=1}^{n} \operatorname{div}\left(f_{i}\right) \in Z_{0}^{+}\left(\mathbb{P}^{1} \times \mathbb{P}^{n-1} ; \mathbb{Q}\right)$. By Corollary 2.62,

$$
[Z]_{\mathbb{Z}} \leq \prod_{i=1}^{n}\left[\operatorname{div}\left(f_{i}\right)\right]_{\mathrm{sup}}
$$


We have that $[Z]_{\mathbb{Z}}=\widehat{\mathrm{h}}_{(1,0)}(Z) \eta \theta_{2}^{n-1}+\widehat{\mathrm{h}}_{(0,1)}(Z) \eta \theta_{1} \theta_{2}^{n-2}+\operatorname{deg}(Z) \theta_{1} \theta_{2}^{n-1}$ and that $\left[\operatorname{div}\left(f_{i}\right)\right]_{\text {sup }}=\log \left\|f_{i}\right\|_{\text {sup }} \eta+\theta_{1}+\theta_{2}$. By comparing coefficients, it follows that

$$
\widehat{\mathrm{h}}_{(1,0)}(Z) \leq \sum_{i} \log \left\|f_{i}\right\|_{\text {sup }}, \quad \widehat{\mathrm{h}}_{(0,1)}(Z) \leq(n-1) \sum_{i} \log \left\|f_{i}\right\|_{\text {sup }}, \quad \operatorname{deg}(Z) \leq n .
$$

Let $\pi_{1}$ and $\pi_{2}$ denote the projections from $\mathbb{P}^{1} \times \mathbb{P}^{n-1}$ to the first and the second factor, respectively. We have that

$$
Z_{1}:=\left(\pi_{1}\right)_{*} Z \in Z_{0}^{+}\left(\mathbb{P}_{\mathbb{Q}}^{1}\right), \quad Z_{2}:=\left(\pi_{2}\right)_{*} Z \in Z_{0}^{+}\left(\mathbb{P}_{\mathbb{Q}}^{n-1}\right)
$$

are the cycles of eigenvalues and of eigenvectors of $M$, respectively. Applying Proposition 2.64, we deduce that the heights of these cycles satisfy

$$
\widehat{\mathrm{h}}\left(Z_{1}\right) \leq \sum_{i} \log \left\|f_{i}\right\|_{\text {sup }}, \quad \widehat{\mathrm{h}}\left(Z_{2}\right) \leq(n-1) \sum_{i} \log \left\|f_{i}\right\|_{\text {sup }} .
$$

A straightforward application of the arithmetic Bézout inequality for projective cycles (Corollary 2.62) would have given the much worse bound $2^{n-1} \sum_{i} \log \left\|f_{i}\right\|_{\text {sup }}$.

Next result gives the behavior of extended Chow rings and classes with respect to products.

Proposition 2.66. - Let $m_{i} \in \mathbb{Z}_{>0}$ and $\boldsymbol{n}_{i} \in \mathbb{N}^{m_{i}}$ for $i=1,2$. Then

1. $A^{*}\left(\mathbb{P}^{\boldsymbol{n}_{1}} \times \mathbb{P}^{\boldsymbol{n}_{2}} ; \mathbb{Z}\right) \simeq A^{*}\left(\mathbb{P}^{\boldsymbol{n}_{1}} ; \mathbb{Z}\right) \otimes_{\mathbb{R}[\eta]} A^{*}\left(\mathbb{P}^{\boldsymbol{n}_{2}} ; \mathbb{Z}\right)$.

2. Let $X_{i} \in Z_{r_{i}}\left(\mathbb{P}_{\mathbb{Q}}^{\boldsymbol{n}_{i}}\right)$ for $i=1,2$. The above isomorphism identifies $\left[X_{1} \times X_{2}\right]_{\mathbb{Z}}$ with $\left[X_{1}\right]_{\mathbb{Z}} \otimes\left[X_{2}\right]_{\mathbb{Z}}$. In particular, for $\boldsymbol{c}_{i} \in \mathbb{N}^{m_{i}}$ such that $\left|\boldsymbol{c}_{1}\right|+\left|\boldsymbol{c}_{2}\right|=r_{1}+r_{2}+1$,

$$
\widehat{\mathrm{h}}_{\left(\boldsymbol{c}_{1}, \boldsymbol{c}_{2}\right)}\left(X_{1} \times X_{2}\right)=\left\{\begin{array}{cl}
\operatorname{deg}_{\boldsymbol{c}_{1}}\left(X_{1}\right) \widehat{\mathrm{h}}_{\boldsymbol{c}_{2}}\left(X_{2}\right) & \text { if }\left|\boldsymbol{c}_{1}\right|=r_{1},\left|\boldsymbol{c}_{2}\right|=r_{2}+1, \\
\operatorname{deg}_{\boldsymbol{c}_{2}}\left(X_{2}\right) \widehat{\mathrm{h}}_{\boldsymbol{c}_{1}}\left(X_{1}\right) & \text { if }\left|\boldsymbol{c}_{1}\right|=r_{1}+1,\left|\boldsymbol{c}_{2}\right|=r_{2}, \\
0 & \text { otherwise. }
\end{array}\right.
$$

Proof. - (1) This is immediate from the definition of the extended Chow ring.

(2) Consider the case when $\left|\boldsymbol{c}_{1}\right|=r_{1}+1,\left|\boldsymbol{c}_{2}\right|=r_{2}$. By Propositions 1.45 and 2.51(1),

$$
\begin{aligned}
\mathrm{h}_{\boldsymbol{c}}\left([\ell]_{*}\left(X_{1} \times X_{2}\right)\right) & =\operatorname{ph}\left(\operatorname{Res}_{\boldsymbol{e}(\boldsymbol{c})}\left([\ell]_{*} X_{1} \times[\ell]_{*} X_{2}\right)\right) \\
& =\operatorname{ph}\left(\operatorname{Res}_{\boldsymbol{e}\left(\boldsymbol{c}_{1}\right)}\left([\ell]_{*} X_{1}\right)^{\operatorname{deg}_{\boldsymbol{c}_{2}}\left([\ell]_{*} X_{2}\right)}\right)=\ell^{r_{2}} \operatorname{deg}_{\boldsymbol{c}_{2}}\left(X_{2}\right) \mathrm{h}_{\boldsymbol{c}_{1}}\left([\ell]_{*} X_{1}\right) .
\end{aligned}
$$

Hence,

$$
\begin{aligned}
\widehat{\mathrm{h}}_{\boldsymbol{c}}\left(X_{1} \times X_{2}\right)=\lim _{\ell \rightarrow \infty} \ell^{-r_{1}-r_{2}-1} \mathrm{~h}_{\boldsymbol{c}}\left([\ell]_{*}\left(X_{1} \times X_{2}\right)\right) \\
\quad=\operatorname{deg}_{\boldsymbol{c}_{2}}\left(X_{2}\right) \lim _{\ell \rightarrow \infty} \ell^{-r_{1}-1} \mathrm{~h}_{\boldsymbol{c}_{1}}\left([\ell]_{*} X_{1}\right)=\widehat{\mathrm{h}}_{\boldsymbol{c}_{1}}\left(X_{1}\right) \operatorname{deg}_{\boldsymbol{c}_{2}}\left(X_{2}\right) .
\end{aligned}
$$

The other cases can be proved similarly. The equality of classes in the extended Chow ring follows from this together with Proposition 1.19.

Finally, we compute the class in the extended Chow ring of the ruled join of two projective varieties. For $i=1,2$ consider the injective $\mathbb{R}$-linear map $\jmath_{i}: A^{*}\left(\mathbb{P}^{n_{i}} ; \mathbb{Z}\right) \hookrightarrow A^{*}\left(\mathbb{P}^{n_{1}+n_{2}+1} ; \mathbb{Z}\right)$ defined by $\theta^{l} \eta^{b} \mapsto \theta^{l} \eta^{b}$ for $0 \leq l \leq n_{i}$ and $b=0,1$. 
Proposition 2.67. - Let $n_{i} \in \mathbb{N}$ and $X_{i} \in Z_{r_{i}}\left(\mathbb{P}_{\mathbb{Q}}^{n_{i}}\right)$ for $i=1,2$. Then

$$
\left[X_{1} \# X_{2}\right]_{\mathbb{Z}}=\jmath_{1}\left(\left[X_{1}\right]_{\mathbb{Z}}\right) \cdot \jmath_{2}\left(\left[X_{2}\right]_{\mathbb{Z}}\right) \text {. }
$$

In particular, $\widehat{\mathrm{h}}\left(X_{1} \# X_{2}\right)=\operatorname{deg}\left(X_{1}\right) \widehat{\mathrm{h}}\left(X_{2}\right)+\operatorname{deg}\left(X_{2}\right) \widehat{\mathrm{h}}\left(X_{1}\right)$.

Proof. - The equality of classes is equivalent to $\operatorname{deg}\left(X_{1} \# X_{2}\right)=\operatorname{deg}\left(X_{1}\right) \operatorname{deg}\left(X_{2}\right)$ and $\widehat{\mathrm{h}}\left(X_{1} \# X_{2}\right)=\operatorname{deg}\left(X_{1}\right) \widehat{\mathrm{h}}\left(X_{2}\right)+\operatorname{deg}\left(X_{2}\right) \widehat{\mathrm{h}}\left(X_{1}\right)$. The first one is (1.20) while the second one is [31, Prop. 4.9(a)].

\section{The height of the implicit equation}

As an application of the results in the previous sections, we obtain sharp bounds for the exponents and the coefficients of an equation defining the closure of the image of an affine variety under a rational map. We consider separately the cases $K$ a field of functions and $K=\mathbb{Q}$. Besides of their intrinsic interest, these results play a central role in our treatment of the parametric and arithmetic Nullstellensätze.

In the sequel, we will be mostly concerned with affine varieties instead of multiprojective cycles. Given $\boldsymbol{n} \in \mathbb{N}^{m}$, we identify the affine space

$$
\mathbb{A}_{K}^{\boldsymbol{n}}:=\mathbb{A}_{K}^{n_{1}} \times \cdots \times \mathbb{A}_{K}^{n_{m}}
$$

with the dense open subset $\mathbb{P}_{K}^{\boldsymbol{n}} \backslash V\left(x_{1,0}, \ldots, x_{m, 0}\right)$. This identification allows to transfer notions and results from $\mathbb{P}_{K}^{n}$ to $\mathbb{A}_{K}^{n}$. For instance, subvarieties, cycles and divisors of $\mathbb{A}_{K}^{\boldsymbol{n}}$ correspond to the restriction of subvarieties, cycles and divisors of $\mathbb{P}_{K}^{n}$ to this open subset. Thus, we can consider their mixed degrees and heights, which will correspond to the analogous notions for their closure in the corresponding multiprojective space. In particular, the degree and the height of a subvariety of $\mathbb{A}_{K}^{n}$ are respectively defined as the degree and the height of its closure in $\mathbb{P}_{K}^{n}$.

For $n \in \mathbb{N}$, the set of $K$-varieties of $\mathbb{A}_{K}^{n}$ is in one-to-one correspondence with the set of radical ideals of $K\left[x_{1}, \ldots, x_{n}\right]$. For an affine $K$-variety $V \subset \mathbb{A}_{K}^{n}$, we denote by $V(\bar{K})$ the set of closed points of $V_{\bar{K}}$. It can be identified with the subset of $\bar{K}^{n}$ defined by $I(V)$. A polynomial relation is said to hold on $V$ if it holds for every point of $V(\bar{K})$.

Since $\mathbb{Q}$ is a perfect field, a $\mathbb{Q}$-variety of $\mathbb{A}_{\mathbb{Q}}^{n}$ can be identified with a subvariety of $\mathbb{A}^{n}(\overline{\mathbb{Q}})$ defined over $\mathbb{Q}$, see Remark 1.1.

\subsection{The function field case}

Let $k$ be an arbitrary field and consider the groups of variables $\boldsymbol{t}=\left\{t_{1}, \ldots, t_{p}\right\}$ and $\boldsymbol{x}=\left\{x_{1}, \ldots, x_{n}\right\}$. The following result is a parametric analogue of Perron's theorem on the size of an equation of algebraic dependence for $r+1$ polynomials over a variety of dimension $r$.

THeOREM 3.1. - Let $V \subset \mathbb{A}_{k(t)}^{n}$ be a $k(\boldsymbol{t})$-variety of pure dimension $r$ and $q_{1}, \ldots, q_{r+1} \in$ $k[\boldsymbol{t}, \boldsymbol{x}] \backslash k[\boldsymbol{t}]$. Set $d_{j}=\operatorname{deg}_{\boldsymbol{x}}\left(q_{j}\right), h_{j}=\operatorname{deg}_{\boldsymbol{t}}\left(q_{j}\right)$ for $1 \leq j \leq r+1$ and write $\boldsymbol{d}=\left(d_{1}, \ldots, d_{r+1}\right)$, $\boldsymbol{h}=\left(h_{1}, \ldots, h_{r+1}\right)$. Then there exists

$$
E=\sum_{\boldsymbol{a} \in \mathbb{N}^{r+1}} \alpha_{\boldsymbol{a}} \boldsymbol{y}^{\boldsymbol{a}} \in k[\boldsymbol{t}]\left[y_{1}, \ldots, y_{r+1}\right] \backslash\{0\}
$$


satisfying $E\left(q_{1}, \ldots, q_{r+1}\right)=0$ on $V$ and such that, for all $\boldsymbol{a} \in \operatorname{supp}(E)$,

$$
\begin{aligned}
& \text { - }\langle\boldsymbol{d}, \boldsymbol{a}\rangle \leq\left(\prod_{j=1}^{r+1} d_{j}\right) \operatorname{deg}(V), \\
& \text { - } \operatorname{deg}\left(\alpha_{\boldsymbol{a}}\right)+\langle\boldsymbol{h}, \boldsymbol{a}\rangle \leq\left(\prod_{j=1}^{r+1} d_{j}\right)\left(\mathrm{h}(V)+\operatorname{deg}(V) \sum_{\ell=1}^{r+1} \frac{h_{\ell}}{d_{\ell}}\right) .
\end{aligned}
$$

Our proof below follows Jelonek's approach in [14, Thm. 3.3] and heavily relies on the arithmetic intersection theory developed in the previous sections.

Lemma 3.2. - Let $V \subset \mathbb{A}_{k(t)}^{n}$ be a $k(\boldsymbol{t})$-variety of pure dimension $r$ and $l \in \mathbb{N}$. Then

$$
\operatorname{deg}\left(V \times \mathbb{A}_{k(t)}^{l}\right)=\operatorname{deg}(V), \quad \mathrm{h}\left(V \times \mathbb{A}_{k(t)}^{l}\right)=\mathrm{h}(V) .
$$

Proof. - Consider the standard inclusions $\iota_{1}, \iota_{2}$ and $\iota_{3}$ of $\mathbb{A}^{n}, \mathbb{A}^{l-1}$ and $\mathbb{A}^{n+l}$ into $\mathbb{P}^{n}$, $\mathbb{P}^{l-1}$ and $\mathbb{P}^{n+l}$, respectively. We can easily verify that $\iota_{3}\left(V \times \mathbb{A}^{l}\right)=\iota_{1}(V) \# \iota_{2}\left(\mathbb{A}^{l-1}\right)$. Applying Propositions 2.24 and 2.13(2),

$$
\left[V \times \mathbb{A}^{l}\right]_{k(t)}=[V]_{k(t)} \cdot\left[\mathbb{A}^{l-1}\right]_{k(t)}=[V]_{k(t)},
$$

which implies the statement.

Proof of Theorem 3.1. - Consider first the case when the map

$$
\psi: V \longrightarrow \mathbb{A}_{k(t)}^{r+1}, \quad \boldsymbol{x} \longmapsto\left(q_{1}(\boldsymbol{x}), \ldots, q_{r+1}(\boldsymbol{x})\right)
$$

is generically finite onto its image, that is, when the fiber of a generic point in $\operatorname{Im}(\psi)$ is finite. $\operatorname{In}$ our setting, this implies that $\overline{\operatorname{Im}(\psi)}$ is a hypersurface. Let $E \in k[\boldsymbol{t}][\boldsymbol{y}] \backslash\{0\}$ be a primitive and squarefree polynomial defining this hypersurface. Let $v, w_{1}, \ldots, w_{r+1}$ be a set of auxiliary variables and write

$$
P=E\left(w_{1}+v^{h_{1}} y_{1}^{d_{1}}, \ldots, w_{r+1}+v^{h_{r+1}} y_{r+1}^{d_{r+1}}\right) \in k[\boldsymbol{t}, v][\boldsymbol{w}, \boldsymbol{y}] .
$$

Hence,

$$
\max _{\boldsymbol{a}}\{\langle\boldsymbol{d}, \boldsymbol{a}\rangle\}=\operatorname{deg}_{\boldsymbol{w}, \boldsymbol{y}}(P), \quad \max _{\boldsymbol{a}}\left\{\operatorname{deg}\left(\alpha_{\boldsymbol{a}}\right)+\langle\boldsymbol{h}, \boldsymbol{a}\rangle\right\}=\operatorname{deg}_{\boldsymbol{t}, v}(P) .
$$

We have that $E$ is primitive and squarefree as a polynomial in $k[\boldsymbol{t}, v][\boldsymbol{w}, \boldsymbol{y}]$. Hence the same holds for $P$, since the map defined by $y_{j} \mapsto w_{j}+v^{h_{j}} y_{j}^{d_{j}}, w_{j} \mapsto y_{j}$ is the identity on $k[\boldsymbol{t}, v]$ and an automorphism of $k[\boldsymbol{t}, v][\boldsymbol{w}, \boldsymbol{y}]$ which sends $P$ to $E$.

Write $V(P)$ for the $k(\boldsymbol{t}, v)$-hypersurface of $\mathbb{A}_{k(\boldsymbol{t}, v)}^{2 r+2}$ defined by $P$. By Proposition 2.13(4),

$$
\operatorname{deg}_{\boldsymbol{w}, \boldsymbol{y}}(P)=\operatorname{deg}(V(P)), \quad \operatorname{deg}_{\boldsymbol{t}, v}(P)=\mathrm{h}(V(P)) .
$$

We will bound both the degree and the height of this hypersurface. Consider the following subvarieties of $\mathbb{A}_{k(t, v)}^{n+2 r+2}$ :

$$
G=\left(V \times \mathbb{A}^{2 r+2}\right) \cap \bigcap_{j=1}^{r+1} V\left(y_{j}-q_{j}\right), \quad G(\boldsymbol{d}, \boldsymbol{h})=\left(V \times \mathbb{A}^{2 r+2}\right) \cap \bigcap_{j=1}^{r+1} V\left(w_{j}+v^{h_{j}} y_{j}^{d_{j}}-q_{j}\right) .
$$

Both varieties are of pure dimension $2 r+1$ : indeed, each of them is defined by a set of $r+1$ polynomials which form a complete intersection over $V \times \mathbb{A}^{2 r+2}$ as they depend on different 
variables $y_{j}$. Let $\rho: \mathbb{A}^{r+1} \rightarrow \mathbb{A}^{r+1}$ be the map defined by $y_{j} \mapsto w_{j}+v^{h_{j}} y_{j}^{d_{j}}$ and $\pi$ the projection $\mathbb{A}^{n+2 r+2} \simeq \mathbb{A}^{n} \times \mathbb{A}^{2 r+2} \rightarrow \mathbb{A}^{2 r+2}$. We have a commutative diagram

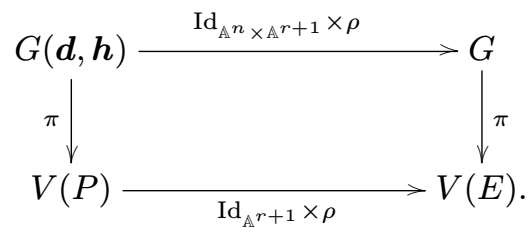

Both $G(\boldsymbol{d}, \boldsymbol{h}) \rightarrow G$ and $G \rightarrow V(E)$ are generically finite since $\psi$ is generically finite, and so this is also the case for $\pi: G(\boldsymbol{d}, \boldsymbol{h}) \rightarrow V(P)$. Proposition 2.22 then implies that

$$
\operatorname{deg}(V(P)) \leq \operatorname{deg}(G(\boldsymbol{d}, \boldsymbol{h})), \quad \mathrm{h}(V(P)) \leq \mathrm{h}(G(\boldsymbol{d}, \boldsymbol{h})) .
$$

Observe that $\operatorname{deg}_{\boldsymbol{x}, \boldsymbol{w}, \boldsymbol{y}}\left(v^{h_{j}} y_{j}^{d_{j}}+w_{j}-q_{j}\right)=d_{j}$ and $\operatorname{deg}_{\boldsymbol{t}, v}\left(v^{h_{j}} y_{j}^{d_{j}}+w_{j}-q_{j}\right)=h_{j}$. Hence, since $G(\boldsymbol{d}, \boldsymbol{h})$ is an open set of a component defined by the homogenization of these equations in $\mathbb{P}^{n+2 r+2}$,

$$
\begin{aligned}
\operatorname{deg}(G(\boldsymbol{d}, \boldsymbol{h})) & \leq\left(\prod_{j=1}^{r+1} d_{j}\right) \operatorname{deg}\left(V \times \mathbb{A}^{2 r+2}\right)=\left(\prod_{j=1}^{r+1} d_{j}\right) \operatorname{deg}(V), \\
\mathrm{h}(G(\boldsymbol{d}, \boldsymbol{h})) & \leq\left(\prod_{j=1}^{r+1} d_{j}\right)\left(\mathrm{h}\left(V \times \mathbb{A}^{2 r+2}\right)+\operatorname{deg}\left(V \times \mathbb{A}^{2 r+2}\right) \sum_{\ell=1}^{r+1} \frac{h_{\ell}}{d_{\ell}}\right) \\
& \leq\left(\prod_{j=1}^{r+1} d_{j}\right)\left(\mathrm{h}(V)+\operatorname{deg}(V) \sum_{\ell=1}^{r+1} \frac{h_{\ell}}{d_{\ell}}\right),
\end{aligned}
$$

thanks to Corollary 2.19 and Lemma 3.2. The statements follows from these bounds, together with (3.3), (3.4) and (3.5).

The general case reduces to the generically finite one by a deformation argument. Choose variables $x_{i_{1}}, \ldots, x_{i_{r+1}}$ among those in the group $\boldsymbol{x}$ in such a way that the projection $V \rightarrow \mathbb{A}_{k(t)}^{r+1}, \boldsymbol{x} \mapsto\left(x_{i_{1}}, \ldots, x_{i_{r+1}}\right)$ is generically finite onto its image. Adding a further variable $z$, we consider the map

$$
V_{k(z, \boldsymbol{t})} \longrightarrow \mathbb{A}_{k(z, t)}^{r+1}, \quad \boldsymbol{x} \longmapsto\left(q_{1}(\boldsymbol{x})+z x_{i_{1}}, \ldots, q_{r+1}(\boldsymbol{x})+z x_{i_{r+1}}\right) .
$$

It is also generically finite onto its image. Thus, we are in the hypothesis of the previous case with respect to the base ring $k(z)[\boldsymbol{t}]$. We deduce that there is a polynomial $\widetilde{E}=\sum_{\boldsymbol{a}} \widetilde{\alpha}_{\boldsymbol{a}} \boldsymbol{y}^{\boldsymbol{a}} \in$ $k(z)[\boldsymbol{t}][\boldsymbol{y}] \backslash\{0\}$ defining the closure of the image of this map and satisfying, for all $\boldsymbol{a} \in \operatorname{supp}(\widetilde{E})$,

$$
\langle\boldsymbol{d}, \boldsymbol{a}\rangle \leq\left(\prod_{j=1}^{r+1} d_{j}\right) \operatorname{deg}(V), \quad \operatorname{deg}_{\boldsymbol{t}}\left(\widetilde{\alpha}_{\boldsymbol{a}}\right)+\langle\boldsymbol{h}, \boldsymbol{a}\rangle \leq\left(\prod_{j=1}^{r+1} d_{j}\right)\left(\mathrm{h}(V)+\operatorname{deg}(V) \sum_{\ell=1}^{r+1} \frac{h_{\ell}}{d_{\ell}}\right) .
$$

After multiplying by a suitable non-zero polynomial in $z$, we can assume without loss of generality that $\widetilde{E}$ lies in $k[z][\boldsymbol{t}, \boldsymbol{y}]$ and that it is primitive as a polynomial in the variables $\boldsymbol{t}, \boldsymbol{y}$. Set $E=\widetilde{E}(0, \boldsymbol{t}, \boldsymbol{y}) \in k[\boldsymbol{t}, \boldsymbol{y}] \backslash\{0\}$. We have that $\widetilde{E}\left(q_{1}+z x_{i_{1}}, \ldots, q_{r+1}+z x_{i_{r+1}}\right) \in$ $I(V) \otimes k(\boldsymbol{t})[z]$ and so $E(\boldsymbol{q}) \in I(V)$ or, equivalently, $E(\boldsymbol{q})=0$ on $V$. We deduce that $E=0$ is an equation of algebraic dependence for $q_{1}, \ldots, q_{r+1}$ which satisfies the same bounds as $\widetilde{E}$. 
For $V=\mathbb{A}_{k(t)}^{n}$ we have $r=n, \operatorname{deg}(V)=1$ and $\mathrm{h}(V)=0$. The above result gives the bounds

$$
\langle\boldsymbol{d}, \boldsymbol{a}\rangle \leq \prod_{j=1}^{n+1} d_{j}, \quad \operatorname{deg}\left(\alpha_{\boldsymbol{a}}\right)+\langle\boldsymbol{h}, \boldsymbol{a}\rangle \leq \sum_{\ell=1}^{n+1}\left(\prod_{j \neq \ell} d_{j}\right) h_{\ell}
$$

for the $\boldsymbol{y}$-degree and the $\boldsymbol{t}$-degree of an equation of algebraic dependence for $n+1$ polynomials in $k[\boldsymbol{t}, \boldsymbol{x}]$ of $\boldsymbol{x}$-degree $d_{j} \geq 1$ and $\boldsymbol{t}$-degree $h_{j}$.

Example 3.7. - Let $d_{1}, d_{2} \in \mathbb{N}$ be coprime integers and set $q_{j}=g_{j} x^{d_{j}}-1 \in k[t, x]$ for a generic univariate polynomial $g_{j} \in k[t]$ of degree $h_{j}, j=1,2$. The implicit equation of the closure of the image of

$$
\mathbb{A}_{k(t)}^{1} \longrightarrow \mathbb{A}_{k(t)}^{2}, \quad x \longmapsto\left(q_{1}(x), q_{2}(x)\right)
$$

is $g_{2}^{d_{1}}\left(y_{1}+1\right)^{d_{2}}-g_{1}^{d_{2}}\left(y_{2}+1\right)^{d_{1}}=0$. The bounds (3.6) are optimal in this case.

Theorem 3.1 can be regarded as an estimate for the Newton polytope of the equation $E$ : if we write $E=\sum_{\boldsymbol{a}, \boldsymbol{c}} \gamma_{\boldsymbol{a}, \boldsymbol{c}} \boldsymbol{t}^{\boldsymbol{c}} \boldsymbol{y}^{\boldsymbol{a}} \in k[\boldsymbol{t}, \boldsymbol{y}]$ with $\gamma_{\boldsymbol{a}, \boldsymbol{c}} \in k$, the corresponding Newton polytope is the convex hull

$$
\mathrm{N}(E)=\operatorname{conv}\left\{(\boldsymbol{a}, \boldsymbol{c}): \gamma_{\boldsymbol{a}, \boldsymbol{c}} \neq 0\right\} \subset \mathbb{R}^{r+1} \times \mathbb{R}^{p} .
$$

Theorem 3.1 is equivalent to the statement that $\mathrm{N}(E)$ is contained in the intersection of the non-negative orthant with the two half-spaces defined by the inequalities

$$
\langle\boldsymbol{d}, \boldsymbol{a}\rangle \leq\left(\prod_{j=1}^{r+1} d_{j}\right) \operatorname{deg}(V), \quad\langle\mathbf{1}, \boldsymbol{c}\rangle+\langle\boldsymbol{h}, \boldsymbol{a}\rangle \leq\left(\prod_{j=1}^{r+1} d_{j}\right)\left(\mathrm{h}(V)+\operatorname{deg}(V) \sum_{\ell=1}^{r+1} \frac{h_{\ell}}{d_{\ell}}\right),
$$

where $\mathbf{1}=(1, \ldots, 1) \in \mathbb{N}^{p}$. Indeed, it can be shown that, for $V=\mathbb{A}_{k(t)}^{n}$ and generic polynomials $q_{j}$ of $\boldsymbol{x}$-degree $d_{j} \geq 1$ and $\boldsymbol{t}$-degree $h_{j}$, the Newton polytope of $E$ coincides with the set cut out by these inequalities.

For instance, consider in Example 3.7 the case when $d_{2} h_{1} \geq d_{1} h_{2}$. Then, the Newton polytope of $E$ is the convex hull of the points $(0,0,0),\left(d_{2}, 0,0\right),\left(d_{2}, 0, d_{1} h_{2}\right),\left(0,0, d_{2} h_{1}\right)$, $\left(0, d_{1}, d_{2} h_{1}\right),\left(0, d_{1}, 0\right)$, as shown in the figure:

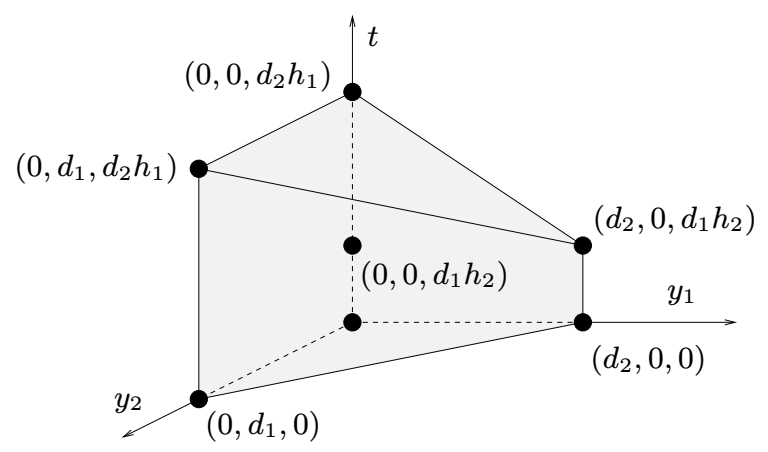

It coincides with the subset of $\mathbb{R}^{3}$ cut out by the inequalities (3.6), namely

$\left\{\left(a_{1}, a_{2}, c\right): \quad a_{1}, a_{2}, c \geq 0, \quad d_{1} a_{1}+d_{2} a_{2} \leq d_{1} d_{2}, \quad c+h_{1} a_{1}+h_{2} a_{2} \leq d_{1} h_{2}+d_{2} h_{1}\right\}$. 
Example 3.8. - Consider the elliptic curve $C=V\left((t+1) x_{1}^{3}+x_{1}^{2}-x_{2}^{2}\right) \subset \mathbb{A}_{k(t)}^{2}$ and the polynomials $q_{1}=x_{1}+(t+1) x_{2}-1, q_{2}=x_{1} x_{2}+(t-1) x_{2}^{2}+t \in k\left[t, x_{1}, x_{2}\right]$. The implicit equation of the closure of the image of

$$
C \longrightarrow \mathbb{A}_{k(t)}^{2}, \quad\left(x_{1}, x_{2}\right) \longmapsto\left(q_{1}\left(x_{1}, x_{2}\right), q_{2}\left(x_{1}, x_{2}\right)\right)
$$

is defined by a polynomial $E \in k\left[t, y_{1}, y_{2}\right]$ with 138 terms. Its Newton polytope is the polytope $\operatorname{conv}((0,0,0),(6,0,0),(6,0,5),(0,0,11),(0,3,8),(0,3,0)) \subset \mathbb{R}^{3}$ :

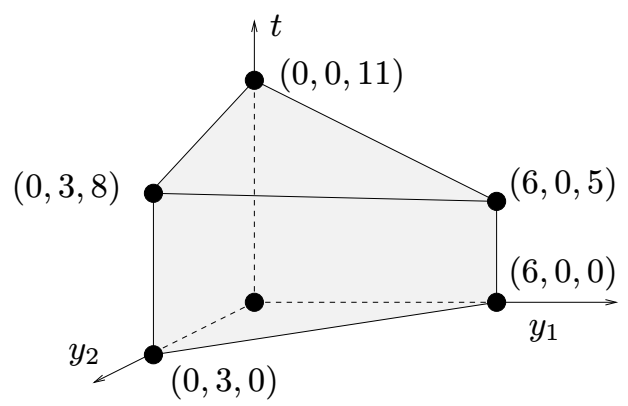

It also coincides with the polytope cut out by the inequalities in the parametric Perron's theorem: we have that $\operatorname{deg}(C)=3$ and $\mathrm{h}(C)=1$ while $\operatorname{deg}_{\boldsymbol{x}}\left(q_{1}\right)=1, \operatorname{deg}_{\boldsymbol{x}}\left(q_{2}\right)=2$ and $\operatorname{deg}_{t}\left(q_{i}\right)=1, i=1,2$. Theorem 3.1 implies the inclusion of polytopes

$$
\mathrm{N}(E) \subset\left\{\left(a_{1}, a_{2}, c\right): \quad a_{1}, a_{2}, c \geq 0, \quad a_{1}+2 a_{2} \leq 6, \quad c+a_{1}+a_{2} \leq 11\right\},
$$

which turns out to be an equality.

The following result is an upper bound for the degree and the height of the implicit equation of a hypersurface defined as the closure of the image of a general rational map. These estimates are not used in the proof of the arithmetic Nullstellensätze. Nevertheless, we include them because they may have some independent interest.

Recall that a rational map $\rho: V \rightarrow \mathbb{A}_{k(t)}^{r+1}$ is defined by quotients of polynomials whose denominators do not vanish identically on any of the components of $V$. It is generically finite onto its image if the fiber of a generic point in $\operatorname{Im}(\rho)$ is finite. In our setting, this implies that the closure of its image is a hypersurface.

THeOREM 3.9. - Let $V \subset \mathbb{A}_{k(t)}^{n}$ be a $k(\boldsymbol{t})$-variety of pure dimension $r$ and

$$
\psi: V \rightarrow \mathbb{A}_{k(t)}^{r+1}, \quad \boldsymbol{x} \longmapsto\left(\frac{q_{1}}{p_{1}}(\boldsymbol{x}), \ldots, \frac{q_{r+1}}{p_{r+1}}(\boldsymbol{x})\right)
$$

a rational map, generically finite onto its image, defined by polynomials $q_{j}, p_{j} \in k[\boldsymbol{t}, \boldsymbol{x}]$ such that $q_{j} / p_{j} \notin k(\boldsymbol{t})$. Let $E \in k[\boldsymbol{t}, \boldsymbol{y}]$ be a primitive and squarefree polynomial defining $\overline{\operatorname{Im}(\psi)}$. Set $d_{j}=\max \left\{\operatorname{deg}_{\boldsymbol{x}}\left(q_{j}\right), \operatorname{deg}_{\boldsymbol{x}}\left(p_{j}\right)\right\}$ and $h_{j}=\max \left\{\operatorname{deg}_{\boldsymbol{t}}\left(q_{j}\right), \operatorname{deg}_{\boldsymbol{t}}\left(p_{j}\right)\right\}$ for $1 \leq j \leq r+1$. Then

$$
\begin{aligned}
& \text { - } \operatorname{deg}_{y_{i}}(E) \leq\left(\prod_{j \neq i} d_{j}\right) \operatorname{deg}(V) \quad \text { for } 1 \leq i \leq r+1, \\
& \text { - } \operatorname{deg}_{t}(E) \leq\left(\prod_{j=1}^{r+1} d_{j}\right)\left(\mathrm{h}(V)+\operatorname{deg}(V) \sum_{\ell=1}^{r+1} \frac{h_{\ell}}{d_{\ell}}\right)
\end{aligned}
$$


Proof. - Let $U=V \backslash \bigcup_{j=1}^{r+1} V\left(p_{j}\right)$ be the dense open subset of $V$ where $\psi$ is defined. The graph of $\psi$ is

$$
\left\{(\boldsymbol{x}, \boldsymbol{y}): \boldsymbol{x} \in U, y_{j} p_{j}(\boldsymbol{x})=q_{j}(\boldsymbol{x}) \text { for } 1 \leq j \leq r+1\right\} \subset U \times \mathbb{A}^{r+1}(\overline{k(\boldsymbol{t})}) .
$$

Let $G$ be the closure of this set in $V \times \mathbb{A}_{k(t)}^{r+1}$. The equations $y_{j} p_{j}=q_{j}$ intersect properly on $U \times \mathbb{A}_{k(t)}^{r+1}$ because they depend on different variables $y_{j}$. Hence, $G$ is an equidimensional variety of dimension $r$, and the projection $V \times \mathbb{A}^{r+1} \rightarrow \mathbb{A}^{r+1}$ induces a generically finite map between $G$ and $V(E)$.

We consider the mixed degrees and heights of $G$ and $V(E)$ with respect to the inclusions

$$
V \times \mathbb{A}^{r+1} \hookrightarrow \mathbb{P}^{n} \times\left(\mathbb{P}^{1}\right)^{r+1}, \quad \mathbb{A}^{r+1} \hookrightarrow\left(\mathbb{P}^{1}\right)^{r+1} .
$$

Set $\mathbf{1}=(1, \ldots, 1) \in \mathbb{N}^{r+1}$ and let $\boldsymbol{e}_{i}$ denote the $i$-th vector of the standard basis of $\mathbb{R}^{r+1}$. By Proposition 2.13(4),

$$
\operatorname{deg}_{y_{i}}(E)=\operatorname{deg}_{\mathbf{1}-\boldsymbol{e}_{i}}(V(E)), \quad \operatorname{deg}_{\boldsymbol{t}}(E)=\mathrm{h}_{\mathbf{1}}(V(E)) .
$$

Proposition 2.22 applied to the projection $\mathbb{P}^{n} \times\left(\mathbb{P}^{1}\right)^{r+1} \rightarrow\left(\mathbb{P}^{1}\right)^{r+1}$ implies that

$$
\operatorname{deg}_{\mathbf{1}-\boldsymbol{e}_{i}}(V(E)) \leq \operatorname{deg}_{0, \mathbf{1}-\boldsymbol{e}_{i}}(G), \quad \mathrm{h}_{\mathbf{1}}(V(E)) \leq \mathrm{h}_{0, \mathbf{1}}(G) .
$$

Let $[G]_{k[t]} \in \mathbb{Z}\left[\eta, \theta_{0}, \boldsymbol{\theta}\right] /\left(\eta^{2}, \theta_{0}^{n+1}, \theta_{1}^{2}, \ldots, \theta_{r+1}^{2}\right)$ be the class of $G$ in the extended Chow ring of $\mathbb{P}^{n} \times\left(\mathbb{P}^{1}\right)^{r+1}$. Observe that $G$ is contained in the part of dimension $r$ of the intersection

$$
\left(V \times \mathbb{A}^{r+1}\right) \cap \bigcap_{j=1}^{r+1} V\left(y_{j} p_{j}-q_{j}\right) .
$$

We will give an upper bound for the class of $G$ by applying Corollary 2.19 recursively to the variety $V \times \mathbb{A}^{r+1}$ and the polynomials $g_{j}=y_{j} p_{j}-q_{j}$ for $j=1, \ldots, r+1$. By Propositions 2.23 and 2.13(2),

$$
\left[V \times \mathbb{A}^{r+1}\right]_{k[t]}=[V]_{k[t]} \otimes\left[\mathbb{A}^{1}\right]_{k[t]}^{\otimes(r+1)}=[V]_{k[t]}=\mathrm{h}(V) \eta \theta_{0}^{n-r-1}+\operatorname{deg}(V) \theta_{0}^{n-r} .
$$

Hence,

$$
\begin{aligned}
{[G]_{k[t]} } & \leq\left[V \times \mathbb{A}^{r+1}\right]_{k[t]} \prod_{j=1}^{r+1}\left(\operatorname{deg}_{\boldsymbol{t}}\left(g_{j}\right) \eta+\operatorname{deg}_{\boldsymbol{x}}\left(g_{j}\right) \theta_{0}+\sum_{\ell=1}^{r+1} \operatorname{deg}_{y_{\ell}}\left(g_{j}\right) \theta_{\ell}\right) \\
& =\left(\mathrm{h}(V) \eta \theta_{0}^{n-r-1}+\operatorname{deg}(V) \theta_{0}^{n-r}\right) \prod_{j=1}^{r+1}\left(h_{j} \eta+d_{j} \theta_{0}+\theta_{j}\right) .
\end{aligned}
$$

Observe that $\mathrm{h}_{0, \mathbf{1}}(G)$ and $\operatorname{deg}_{0, \mathbf{1}-\boldsymbol{e}_{i}}(G)$ are the coefficients in $[G]_{k[t]}$ of the monomials $\eta \theta_{0}^{n}$ and $\theta_{0}^{n} \theta_{i}$, respectively. Therefore

$$
\operatorname{deg}_{0, \mathbf{1}-\boldsymbol{e}_{i}}(G) \leq\left(\prod_{j \neq i} d_{j}\right) \operatorname{deg}(V), \quad \mathrm{h}_{0, \mathbf{1}}(G) \leq\left(\prod_{j=1}^{r+1} d_{j}\right)\left(\mathrm{h}(V)+\operatorname{deg}(V) \sum_{\ell=1}^{r+1} \frac{h_{\ell}}{d_{\ell}}\right) .
$$

The statement follows from these estimates, together with (3.10) and (3.11). 
For $V=\mathbb{A}_{k(t)}^{n}$, the above result gives the bounds

$$
\operatorname{deg}_{y_{i}}(E) \leq \prod_{j \neq i} d_{j}, \quad \operatorname{deg}_{\boldsymbol{t}}(E) \leq \sum_{\ell=1}^{n+1}\left(\prod_{j \neq \ell} d_{j}\right) h_{\ell} .
$$

It can be shown that, for generic polynomials of $\boldsymbol{x}$-degree $d_{j}$ and $\boldsymbol{t}$-degree $h_{j}$, the Newton polytope of $E$ coincides with the subset of $\mathbb{R}^{r+1} \times \mathbb{R}^{p}$ cut out by these inequalities.

ExAmple 3.13. - Let $d_{1}, d_{2} \in \mathbb{N}$ be coprime integers and $g_{i} \in k[t]$ univariate polynomials of degree $h_{i}, i=1,2$. Set

$$
\frac{q_{1}}{p_{1}}=\frac{x^{d_{1}}}{(x+1)^{d_{1}} g_{1}}-1, \quad \frac{q_{2}}{p_{2}}=\frac{(x+1)^{d_{2}}}{x^{d_{2}} g_{2}}-1 .
$$

The implicit equation of the closure of the image of the map

$$
\mathbb{A}_{k(t)}^{1} \rightarrow \mathbb{A}_{k(t)}^{2}, \quad x \longmapsto\left(\frac{q_{1}}{p_{1}}(x), \frac{q_{2}}{p_{2}}(x)\right)
$$

is given by the polynomial $E=g_{1}{ }^{d_{2}} g_{2}{ }^{d_{1}}\left(y_{1}+1\right)^{d_{2}}\left(y_{2}+1\right)^{d_{1}}-1 \in k\left[t, y_{1}, y_{2}\right]$. Theorem 3.9 is optimal in this case, since $\operatorname{deg}_{y_{1}}(E)=d_{2}, \operatorname{deg}_{y_{2}}(E)=d_{1}$ and $\operatorname{deg}_{t}(E)=d_{1} h_{2}+d_{2} h_{1}$. Moreover, we can check that the Newton polytope of $E$ coincides with the set cut out by the inequalities in (3.12).

The previous results can be extended to polynomials depending on groups of parameters. For the sequel, we will need the multiparametric version of Theorem 3.1 that we state below. Let $\boldsymbol{t}_{l}=\left\{t_{l, 1}, \ldots, t_{l, p_{l}}\right\}$ be a group of variables for $1 \leq l \leq m$ and set $\boldsymbol{t}=\left\{\boldsymbol{t}_{1}, \ldots, \boldsymbol{t}_{m}\right\}$. For each $1 \leq l \leq m$, write $k_{l}=k\left(\boldsymbol{t}_{1}, \ldots, \boldsymbol{t}_{l-1}, \boldsymbol{t}_{l+1}, \ldots, \boldsymbol{t}_{m}\right)$ and observe that $k_{l}\left(\boldsymbol{t}_{l}\right)=k(\boldsymbol{t})$. Hence, for a given projective $k(\boldsymbol{t})$-variety $V$, we can consider its height with respect to the base ring $k_{l}\left[\boldsymbol{t}_{l}\right]$. We denote this height $\mathrm{h}_{\boldsymbol{t}_{l}}(V)$.

COROLlary 3.14. - Let $V \subset \mathbb{A}_{k(\boldsymbol{t})}^{n}$ be a $k(\boldsymbol{t})$-variety of pure dimension $r$ and $q_{1}, \ldots, q_{r+1} \in k[\boldsymbol{t}][\boldsymbol{x}] \backslash k[\boldsymbol{t}]$ such that the map

$$
\psi: V \longrightarrow \mathbb{A}^{r+1}, \quad \boldsymbol{x} \longmapsto\left(q_{1}(\boldsymbol{x}), \ldots, q_{r+1}(\boldsymbol{x})\right)
$$

is generically finite onto its image. Let $E=\sum_{\boldsymbol{a} \in \mathbb{N}^{r+1}} \alpha_{\boldsymbol{a}} \boldsymbol{y}^{\boldsymbol{a}} \in k[\boldsymbol{t}]\left[y_{1}, \ldots, y_{r+1}\right]$ be a primitive and squarefree polynomial defining $\overline{\operatorname{Im}(\psi)}$. Set $d_{j}=\operatorname{deg}_{\boldsymbol{x}}\left(q_{j}\right), h_{l, j}=\operatorname{deg}_{\boldsymbol{t}_{l}}\left(q_{j}\right)$ for $1 \leq j \leq r+1,1 \leq l \leq m$ and write $\boldsymbol{d}=\left(d_{1}, \ldots, d_{r+1}\right), \boldsymbol{h}_{l}=\left(h_{l, 1}, \ldots, h_{l, r+1}\right)$. Then, for all $\boldsymbol{a} \in \operatorname{supp}(E)$,

$$
\begin{aligned}
& \text { - }\langle\boldsymbol{d}, \boldsymbol{a}\rangle \leq\left(\prod_{j=1}^{r+1} d_{j}\right) \operatorname{deg}(V), \\
& \text { - } \operatorname{deg}_{\boldsymbol{t}_{l}}\left(\alpha_{\boldsymbol{a}}\right)+\left\langle\boldsymbol{h}_{l}, \boldsymbol{a}\right\rangle \leq\left(\prod_{j=1}^{r+1} d_{j}\right)\left(\mathrm{h}_{\boldsymbol{t}_{l}}(V)+\operatorname{deg}(V) \sum_{\ell=1}^{r+1} \frac{h_{l, \ell}}{d_{\ell}}\right) \quad \text { for } 1 \leq l \leq m .
\end{aligned}
$$

Proof. - Observe that $E$ is primitive and squarefree as an element of $k_{l}\left[\boldsymbol{t}_{l}\right][\boldsymbol{y}]$. The result then follows from Theorem 3.1 applied to the field $k_{l}$ and the group of variables $\boldsymbol{t}_{l}$. 


\subsection{The rational case}

We now turn our attention to the problem of estimating the size of the implicit equation for a rational map defined over $\mathbb{Q}$. It will be convenient to consider a more general situation where the input polynomials depend on groups of parameters. As in the end of the previous section, we set $\boldsymbol{t}_{l}=\left\{t_{l, 1}, \ldots, t_{l, p_{l}}\right\}$ for $1 \leq l \leq m$ and $\boldsymbol{t}=\left\{\boldsymbol{t}_{1}, \ldots, \boldsymbol{t}_{m}\right\}$.

Theorem 3.15. - Let $V \subset \mathbb{A}_{\mathbb{Q}}^{n}$ be a $\mathbb{Q}$-variety of pure dimension $r$ and $q_{1}, \ldots, q_{r+1} \in \mathbb{Z}[\boldsymbol{t}][\boldsymbol{x}] \backslash \mathbb{Z}[\boldsymbol{t}]$ such that the map

$$
\psi: V_{\mathbb{Q}(t)} \longrightarrow \mathbb{A}_{\mathbb{Q}(t)}^{r+1}, \quad \boldsymbol{x} \longmapsto\left(q_{1}(\boldsymbol{x}), \ldots, q_{r+1}(\boldsymbol{x})\right)
$$

is generically finite onto its image. Let $E=\sum_{\boldsymbol{a} \in \mathbb{N}^{r+1}} \alpha_{\boldsymbol{a}} \boldsymbol{y}^{\boldsymbol{a}} \in \mathbb{Z}[\boldsymbol{t}]\left[y_{1}, \ldots, y_{r+1}\right]$ be a primitive and squarefree polynomial defining $\overline{\operatorname{Im}(\psi)}$. Set $d_{j}=\operatorname{deg}_{\boldsymbol{x}}\left(q_{j}\right), \delta_{l, j}=\operatorname{deg}_{\boldsymbol{t}_{l}}\left(q_{j}\right), h_{j}=\mathrm{h}\left(q_{j}\right)$ for $1 \leq j \leq r+1,1 \leq l \leq m$ and write $\boldsymbol{d}=\left(d_{1}, \ldots, d_{r+1}\right), \boldsymbol{\delta}_{l}=\left(\delta_{l, 1}, \ldots, \delta_{l, r+1}\right)$, $\boldsymbol{h}=\left(h_{1}, \ldots, h_{r+1}\right)$. Then, for all $\boldsymbol{a} \in \operatorname{supp}(E)$,

$$
\begin{aligned}
& \bullet\langle\boldsymbol{d}, \boldsymbol{a}\rangle \leq\left(\prod_{j=1}^{r+1} d_{j}\right) \operatorname{deg}(V), \\
& \text { - } \operatorname{deg}_{\boldsymbol{t}_{l}}\left(\alpha_{\boldsymbol{a}}\right)+\left\langle\boldsymbol{\delta}_{l}, \boldsymbol{a}\right\rangle \leq\left(\prod_{j=1}^{r+1} d_{j}\right) \operatorname{deg}(V) \sum_{\ell=1}^{r+1} \frac{\delta_{l, \ell}}{d_{\ell}} \quad \text { for } 1 \leq l \leq m, \\
& \bullet \mathrm{h}\left(\alpha_{\boldsymbol{a}}\right)+\langle\boldsymbol{h}, \boldsymbol{a}\rangle \leq\left(\prod_{j=1}^{r+1} d_{j}\right)(\widehat{\mathrm{h}}(V)+\operatorname{deg}(V)(\log (r+2) \\
&\left.\left.\quad+\sum_{\ell=1}^{r+1} \frac{1}{d_{\ell}}\left(h_{\ell}+\log \left(\# \operatorname{supp}\left(q_{\ell}\right)+2\right)+\sum_{l=1}^{m} \delta_{l, \ell} \log \left(p_{l}+1\right)\right)\right)\right) .
\end{aligned}
$$

We need the following lemma for the proof of this result. It is the analogue for $\mathbb{Q}$ of Lemma 3.2 and can be proved using the same arguments and Proposition 2.67 instead of 2.24 .

Lemma 3.16. - Let $V \subset \mathbb{A}_{\mathbb{Q}}^{n}$ be a $\mathbb{Q}$-variety of pure dimension $r$ and $l \in \mathbb{N}$. Then

$$
\operatorname{deg}\left(V \times \mathbb{A}_{\mathbb{Q}}^{l}\right)=\operatorname{deg}(V), \quad \widehat{\mathrm{h}}\left(V \times \mathbb{A}_{\mathbb{Q}}^{l}\right)=\widehat{\mathrm{h}}(V) .
$$

Proof of Theorem 3.15. - By Proposition 2.13(2), the height $h_{\boldsymbol{t}_{l}}$ of $V_{\mathbb{Q}(\boldsymbol{t})}$ with respect to the base ring $\mathbb{Q}\left(\boldsymbol{t}_{1}, \ldots, \boldsymbol{t}_{l-1}, \boldsymbol{t}_{l+1}, \ldots, \boldsymbol{t}_{m}\right)\left[\boldsymbol{t}_{l}\right]$ is zero. Then, the bounds for the degrees are a direct consequence of Corollary 3.14. For the height, the proof follows closely the lines of that of Theorem 3.1 with $\mathbb{Z}$ instead of $k[\boldsymbol{t}]$. We will avoid repeating the same verifications when they follow mutatis mutandis the parametric case.

Let $w_{1}, \ldots, w_{r+1}$ be a group of variables and consider the polynomial

$$
P=E\left(w_{1}+H_{1} y_{1}, \ldots, w_{r+1}+H_{r+1} y_{r+1}\right) \in \mathbb{Z}[\boldsymbol{t}, \boldsymbol{w}, \boldsymbol{y}],
$$

with $H_{j}=\mathrm{e}^{h_{j}} \in \mathbb{N}$. It verifies

$$
\max _{\boldsymbol{a}}\left\{\mathrm{h}\left(\alpha_{\boldsymbol{a}}\right)+\langle\boldsymbol{h}, \boldsymbol{a}\rangle\right\}=\mathrm{h}(P(\mathbf{0}, \boldsymbol{y})) .
$$


Applying successively Lemmas 2.32(2) and 2.31(4),

$$
\begin{aligned}
\mathrm{h}(P(\mathbf{0}, \boldsymbol{y})) & \leq \mathrm{m}(P(\mathbf{0}, \boldsymbol{y}))+\operatorname{deg}_{\boldsymbol{y}}(P(\mathbf{0}, \boldsymbol{y})) \log (r+2)+\sum_{l=1}^{m} \operatorname{deg}_{\boldsymbol{t}_{l}}(P(\mathbf{0}, \boldsymbol{y})) \log \left(p_{l}+1\right) \\
& \leq \mathrm{m}(P)+\operatorname{deg}_{\boldsymbol{y}}(E) \log (r+2)+\sum_{l=1}^{m} \operatorname{deg}_{\boldsymbol{t}_{l}}(E) \log \left(p_{l}+1\right) .
\end{aligned}
$$

The polynomial $P$ is primitive and squarefree. Hence, it gives a defining equation for the $\mathbb{Q}$-hypersurface $V(P) \subset \mathbb{A}_{\mathbb{Q}}^{\boldsymbol{p}} \times \mathbb{A}_{\mathbb{Q}}^{2 r+2}$, where $\boldsymbol{p}:=\left(p_{1}, \ldots, p_{m}\right)$. Considering the standard inclusion of this affine space into $\mathbb{P}_{\mathbb{Q}}^{p} \times \mathbb{P}_{\mathbb{Q}}^{2 r+2}$, Proposition 2.53(2) implies

$$
\mathrm{m}(P)=\widehat{\mathrm{h}}_{\boldsymbol{p}, 2 r+2}(V(P)) .
$$

Consider the variety

$$
G(\boldsymbol{d}, \boldsymbol{h})=\left(\mathbb{A}^{\boldsymbol{p}} \times V \times \mathbb{A}^{2 r+2}\right) \cap \bigcap_{j=1}^{r+1} V\left(w_{j}+H_{j} y_{j}-q_{j}\right) \subset \mathbb{A}^{\boldsymbol{p}} \times \mathbb{A}^{n+2 r+2} .
$$

As in the proof of Theorem 3.1, we can verify that it is of pure dimension $|\boldsymbol{p}|+2 r+1$ and that the projection $\mathbb{A}^{\boldsymbol{p}} \times \mathbb{A}^{n+2 r+2} \rightarrow \mathbb{A}^{\boldsymbol{p}} \times \mathbb{A}^{2 r+2}$ induces a generically finite map $\pi: G(\boldsymbol{d}, \boldsymbol{h}) \rightarrow V(P)$. Considering mixed heights with respect to the standard inclusion $\mathbb{A}^{\boldsymbol{p}} \times \mathbb{A}^{n+2 r+2} \hookrightarrow \mathbb{P}^{\boldsymbol{p}} \times \mathbb{P}^{n+2 r+2}$, Proposition 2.64 implies

$$
\widehat{\mathrm{h}}_{\boldsymbol{p}, 2 r+2}(V(P)) \leq \widehat{\mathrm{h}}_{\boldsymbol{p}, 2 r+2}(G(\boldsymbol{d}, \boldsymbol{h})) .
$$

The closure of $\mathbb{A}^{\boldsymbol{p}} \times V \times \mathbb{A}^{2 r+2}$ is $\mathbb{P}^{\boldsymbol{p}} \times \iota\left(V \times \mathbb{A}^{2 r+2}\right)$, where $\iota$ denotes the standard inclusion $\mathbb{A}^{n+2 r+2} \hookrightarrow \mathbb{P}^{n+2 r+2}$. We will consider classes in the extended Chow ring

$$
A^{*}\left(\mathbb{P}^{\boldsymbol{p}} \times \mathbb{P}^{n+2 r+2} ; \mathbb{Z}\right)=\mathbb{R}[\eta, \boldsymbol{\theta}, \zeta] /\left(\eta^{2}, \theta_{1}^{p_{1}+1}, \ldots, \theta_{l}^{p_{l}+1}, \zeta^{n+2 r+3}\right) .
$$

With this notation, Propositions 2.66(2) and 2.51(3) together with Lemma 3.16 imply

$$
\left[\mathbb{A}^{p} \times V \times \mathbb{A}^{2 r+2}\right]_{\mathbb{Z}}=\left[\mathbb{A}^{\boldsymbol{p}}\right]_{\mathbb{Z}} \otimes\left[V \times \mathbb{A}^{2 r+2}\right]_{\mathbb{Z}}=[V]_{\mathbb{Z}}=\widehat{\mathrm{h}}(V) \eta \zeta^{n-r-1}+\operatorname{deg}(V) \zeta^{n-r} .
$$

Write $g_{j}=w_{j}+H_{j} y_{j}-q_{j} \in \mathbb{Z}[\boldsymbol{t}, \boldsymbol{x}, \boldsymbol{w}, \boldsymbol{y}]$ and consider the class associated to its sup-norm as defined in (2.57):

$$
\left[g_{j}\right]_{\text {sup }}=\log \left\|g_{j}\right\|_{\sup } \eta+\sum_{l=1}^{m} \operatorname{deg}_{\boldsymbol{t}_{l}}\left(g_{j}\right) \theta_{l}+\operatorname{deg}_{\boldsymbol{x}, \boldsymbol{w}, \boldsymbol{y}}\left(g_{j}\right) \zeta=\log \left\|g_{j}\right\|_{\sup } \eta+\sum_{l=1}^{m} \delta_{l, j} \theta_{l}+d_{j} \zeta .
$$

The divisors defined by these polynomials intersect $\mathbb{A}^{\boldsymbol{p}} \times V \times \mathbb{A}^{2 r+2}$ properly. Applying recursively Corollary 2.61,

$$
\begin{aligned}
{[G(\boldsymbol{d}, \boldsymbol{h})]_{\mathbb{Z}} } & \leq\left[\mathbb{A}^{\boldsymbol{p}} \times V \times \mathbb{A}^{2 r+2}\right]_{\mathbb{Z}} \prod_{j=1}^{r+1}\left[g_{j}\right]_{\text {sup }} \\
& =\left(\widehat{\mathrm{h}}(V) \eta \zeta^{n-r-1}+\operatorname{deg}(V) \zeta^{n-r}\right) \prod_{j=1}^{r+1}\left(\log \left\|g_{j}\right\|_{\text {sup }} \eta+\sum_{l=1}^{m} \delta_{l, j} \theta_{l}+d_{j} \zeta\right) .
\end{aligned}
$$


The mixed height $\widehat{\mathrm{h}}_{\boldsymbol{p}, 2 r+2}(G(\boldsymbol{d}, \boldsymbol{h}))$ is the coefficient of $\eta \zeta^{n}$ in $[G(\boldsymbol{d}, \boldsymbol{h})]_{\mathbb{Z}}$. Furthermore, $\log \left\|g_{\ell}\right\|_{\text {sup }} \leq h_{\ell}+\log \left(\# \operatorname{supp}\left(q_{\ell}\right)+2\right)$ by Lemma 2.32(1). The above inequality implies

$$
\widehat{\mathrm{h}}_{\boldsymbol{p}, 2 r+2}(G(\boldsymbol{d}, \boldsymbol{h})) \leq\left(\prod_{j=1}^{r+1} d_{j}\right)\left(\widehat{\mathrm{h}}(V)+\operatorname{deg}(V) \sum_{\ell=1}^{r+1} \frac{h_{\ell}+\log \left(\# \operatorname{supp}\left(q_{\ell}\right)+2\right)}{d_{\ell}}\right) .
$$

The statement follows from this inequality together with (3.17), (3.18), (3.19), (3.20), and the already considered bounds for the partial degrees of $E$.

Theorem 4 in the introduction follows from the case $m=0$ of this result and the inequality $\# \operatorname{supp}\left(q_{\ell}\right)+2 \leq(n+3)^{d_{\ell}}$.

REMARK 3.21. - Theorem 4 can be regarded as a first bound for the extended Newton polytope of the implicit equation, defined as the convex envelope of the subset

$$
\left\{(\boldsymbol{a}, \lambda): \boldsymbol{a} \in \operatorname{supp}(E), 0 \leq \lambda \leq \mathrm{h}\left(\alpha_{\boldsymbol{a}}\right)\right\} \subset \mathbb{R}^{r+1} \times \mathbb{R} .
$$

Indeed, it would be interesting to have a better understanding of this "arithmetic" polytope in terms of finer invariants of the input polynomials $q_{j}$ like for instance their extended Newton polytope, instead of just their degree and height.

Example 3.22. - Let $d_{1}, d_{2} \in \mathbb{N}$ and $H_{1}, H_{2} \in \mathbb{N}$ be two pairs of coprime integers and set $q_{j}=H_{j} x^{d_{j}} \in \mathbb{Z}[x]$ for $j=1,2$. The implicit equation of the image of the map

$$
\mathbb{A}_{\mathbb{Q}}^{1} \longrightarrow \mathbb{A}_{\mathbb{Q}}^{2}, \quad x \longmapsto\left(q_{1}(x), q_{2}(x)\right)
$$

is given by the polynomial $E=H_{2}^{d_{1}} y_{1}^{d_{2}}-H_{1}^{d_{2}} y_{2}^{d_{1}} \in \mathbb{Z}\left[y_{1}, y_{2}\right]$.

Then, $\max _{\left(a_{1}, a_{2}\right)}\left\{d_{1} a_{1}+d_{2} a_{2}\right\}=d_{1} d_{2}$ and

$$
\max _{\left(a_{1}, a_{2}\right)}\left\{\log \left|\operatorname{coeff}_{\left(a_{1}, a_{2}\right)}(E)\right|+h_{1} a_{1}+h_{2} a_{2}\right\}=d_{1} h_{2}+d_{2} h_{1},
$$

with $h_{j}=\log \left(H_{j}\right)$. Hence, the bounds in Theorem 4 are optimal in this example, up to a term of size $O\left(d_{1} d_{2}\right)$.

Using a deformation argument, we can extend Theorem 3.15 to the case when the map is not generically finite. For simplicity, we will only state this result for polynomials not depending on parameters.

Corollary 3.23. - Let $V \subset \mathbb{A}_{\mathbb{Q}}^{n}$ be a $\mathbb{Q}$-variety of pure dimension $r$ and $q_{1}, \ldots, q_{r+1} \in \mathbb{Z}[\boldsymbol{x}] \backslash \mathbb{Z}$. Set $d_{j}=\operatorname{deg}\left(q_{j}\right)$ and $h_{j}=\mathrm{h}\left(q_{j}\right)$ for $1 \leq j \leq r+1$ and write $\boldsymbol{d}=\left(d_{1}, \ldots, d_{r+1}\right), \boldsymbol{h}=\left(h_{1}, \ldots, h_{r+1}\right)$. Then there exists

$$
E=\sum_{\boldsymbol{a} \in \mathbb{N}^{r+1}} \alpha_{\boldsymbol{a}} \boldsymbol{y}^{\boldsymbol{a}} \in \mathbb{Z}\left[y_{1}, \ldots, y_{r+1}\right] \backslash\{0\}
$$

satisfying $E\left(q_{1}, \ldots, q_{r+1}\right)=0$ on $V$ and such that, for all $\boldsymbol{a} \in \operatorname{supp}(E)$,

$$
\begin{aligned}
& \text { - }\langle\boldsymbol{d}, \boldsymbol{a}\rangle \leq\left(\prod_{j=1}^{r+1} d_{j}\right) \operatorname{deg}(V), \\
& \text { - } \mathrm{h}\left(\alpha_{\boldsymbol{a}}\right)+\langle\boldsymbol{h}, \boldsymbol{a}\rangle \leq\left(\prod_{j=1}^{r+1} d_{j}\right)\left(\widehat{\mathrm{h}}(V)+\operatorname{deg}(V)\left(\sum_{\ell=1}^{r+1} \frac{h_{\ell}}{d_{\ell}}+(r+2) \log (2 n+8)\right)\right) .
\end{aligned}
$$


Proof. - We will follow the arguments in the proof of Theorem 3.1 adapted to this situation. Choose $r+1$ variables $x_{i_{1}}, \ldots, x_{i_{r+1}}$ in the group $\boldsymbol{x}$ so that the linear projection $V \rightarrow \mathbb{A}_{\mathbb{Q}}^{r+1}, \boldsymbol{x} \mapsto\left(x_{i_{1}}, \ldots, x_{i_{r+1}}\right)$ is generically finite onto its image. Adding a further variable $z$, consider the map

$$
V_{\mathbb{Q}(z)} \longrightarrow \mathbb{A}_{\mathbb{Q}(z)}^{r+1}, \quad \boldsymbol{x} \longmapsto\left(q_{1}(\boldsymbol{x})+z x_{i_{1}}, \ldots, q_{r+1}(\boldsymbol{x})+z x_{i_{r+1}}\right),
$$

which is generically finite onto its image. Let $\widetilde{E}=\sum_{\boldsymbol{a}} \alpha_{\boldsymbol{a}} \boldsymbol{y}^{\boldsymbol{a}} \in \mathbb{Z}[z][\boldsymbol{y}]$ be a primitive and squarefree polynomial defining the closure of the image of this map. The polynomials $q_{j}(\boldsymbol{x})+z x_{i_{j}}$ have $\boldsymbol{x}$-degree $d_{j}, z$-degree 1 and height $h_{j}$. Theorem 3.15 applied to this case gives $\langle\boldsymbol{d}, \boldsymbol{a}\rangle \leq\left(\prod_{j=1}^{r+1} d_{j}\right) \operatorname{deg}(V)$ and

$$
\begin{aligned}
\mathrm{h}\left(\alpha_{\boldsymbol{a}}\right)+\langle\boldsymbol{h}, \boldsymbol{a}\rangle \leq\left(\prod_{j=1}^{r+1} d_{j}\right)(\widehat{\mathrm{h}}(V) & +\operatorname{deg}(V)(\log (r+2) \\
& \left.\left.+\sum_{\ell=1}^{r+1} \frac{1}{d_{\ell}}\left(h_{\ell}+\log \left(\# \operatorname{supp}\left(q_{\ell}\right)+3\right)+\log (2)\right)\right)\right) .
\end{aligned}
$$

The polynomial $E:=\widetilde{E}(0, \boldsymbol{y}) \in \mathbb{Z}[\boldsymbol{y}]$ gives a non-trivial relation of algebraic dependence for the $q_{j}$ 's and it satisfies the same degree and height bounds as $\widetilde{E}$. The statement then follows from the inequality $\# \operatorname{supp}\left(q_{\ell}\right)+3 \leq(n+1)^{d_{\ell}}+3 \leq(n+4)^{d_{\ell}}$.

Next result gives an upper bound for the Mahler measure and a fortiori, for the height of the implicit equation for a rational map.

Theorem 3.24. - Let $V \subset \mathbb{A}_{\mathbb{Q}}^{n}$ be $a \mathbb{Q}$-variety of pure dimension $r$ and

$$
\psi: V \rightarrow \mathbb{A}_{\mathbb{Q}}^{r+1}, \quad \boldsymbol{x} \mapsto\left(\frac{q_{1}}{p_{1}}(\boldsymbol{x}), \ldots, \frac{q_{r+1}}{p_{r+1}}(\boldsymbol{x})\right)
$$

a rational map, generically finite onto its image, defined by polynomials $q_{j}, p_{j} \in \mathbb{Z}[\boldsymbol{x}]$ such that $q_{j} / p_{j} \notin \mathbb{Q}$. Let $E \in \mathbb{Z}[\boldsymbol{y}]$ be a primitive and squarefree polynomial defining $\overline{\operatorname{Im}(\psi)}$. Set $d_{j}=\max \left\{\operatorname{deg}_{\boldsymbol{x}}\left(q_{j}\right), \operatorname{deg}_{\boldsymbol{x}}\left(p_{j}\right)\right\}$ and $h_{j}=\log \left(\left\|q_{j}\right\|_{1}+\left\|p_{j}\right\|_{1}\right)$ for $1 \leq j \leq r+1$. Then

$$
\begin{aligned}
& \text { - } \operatorname{deg}_{y_{i}}(E) \leq\left(\prod_{j \neq i} d_{j}\right) \operatorname{deg}(V) \quad \text { for } 1 \leq i \leq r+1, \\
& \text { - } \mathrm{m}(E) \leq\left(\prod_{j=1}^{r+1} d_{j}\right)\left(\widehat{\mathrm{h}}(V)+\operatorname{deg}(V) \sum_{\ell=1}^{r+1} \frac{h_{\ell}}{d_{\ell}}\right) .
\end{aligned}
$$

Proof. - The bounds for the partial degrees of $E$ follow from Theorem 3.9. Thus, we only have to prove the upper bound for the Mahler measure. Let $V(E) \subset \mathbb{A}_{\mathbb{Q}}^{r+1}$ and $G \subset V \times \mathbb{A}_{\mathbb{Q}}^{r+1}$ denote the closure of the image of $\psi$ and of the graph of $\psi$, respectively. We consider mixed heights of $G$ and $V(E)$ with respect to the inclusions

$$
V \times \mathbb{A}^{r+1} \hookrightarrow \mathbb{P}^{n} \times\left(\mathbb{P}^{1}\right)^{r+1}, \quad \mathbb{A}^{r+1} \hookrightarrow\left(\mathbb{P}^{1}\right)^{r+1} .
$$

Propositions 2.53 and 2.64 imply that

$$
\mathrm{m}(E)=\widehat{\mathrm{h}}_{\mathbf{1}}(V(E)) \leq \widehat{\mathrm{h}}_{0, \mathbf{1}}(G) .
$$

Let $[G]_{\mathbb{Z}} \in \mathbb{R}\left[\eta, \theta_{0}, \boldsymbol{\theta}\right] /\left(\eta^{2}, \theta_{0}^{n+1}, \theta_{1}^{2}, \ldots, \theta_{r+1}^{2}\right)$ be the class of the closure of $G$ in the extended Chow ring of $\mathbb{P}^{n} \times\left(\mathbb{P}^{1}\right)^{r+1}$. We will bound it by applying Corollary 2.61 recursively to 
$V \times \mathbb{A}^{r+1}$ and $g_{j}:=y_{j} p_{j}-q_{j}$ for $j=1, \ldots, r+1$. Propositions 2.66(2) and 2.51(3) imply that $\left[V \times \mathbb{A}^{r+1}\right]_{\mathbb{Z}}=\widehat{\mathrm{h}}(V) \eta \theta_{0}^{n-r-1}+\operatorname{deg}(V) \theta_{0}^{n-r}$. Besides, $\left\|p_{j} y_{j}-q_{j}\right\|_{\text {sup }} \leq\left\|p_{j}\right\|_{1}+\left\|q_{j}\right\|_{1}$ and so $\left[p_{j} y_{j}-q_{j}\right]_{\text {sup }} \leq h_{j} \eta+d_{j} \theta_{0}+\theta_{j}$. Hence,

$[G]_{\mathbb{Z}} \leq\left[V \times \mathbb{A}^{r+1}\right]_{\mathbb{Z}} \prod_{j=1}^{r+1}\left[p_{j} y_{j}-q_{j}\right]_{\text {sup }} \leq\left(\widehat{\mathrm{h}}(V) \eta \theta_{0}^{n-r-1}+\operatorname{deg}(V) \theta_{0}^{n-r}\right) \prod_{j=1}^{r+1}\left(h_{j} \eta+d_{j} \theta_{0}+\theta_{j}\right)$.

The mixed height $\widehat{\mathrm{h}}_{0, \mathbf{1}}(G)$ is the coefficient of the monomial $\eta \theta_{0}^{n}$ in $[G]_{\mathbb{Z}}$. The above inequality then implies

$$
\widehat{\mathrm{h}}_{0, \mathbf{1}}(G) \leq\left(\prod_{j=1}^{r+1} d_{j}\right) \widehat{\mathrm{h}}(V)+\operatorname{deg}(V) \sum_{\ell=1}^{r+1}\left(\prod_{j \neq \ell} d_{j}\right) h_{\ell} .
$$

The statement follows from this inequality and (3.25).

For $V=\mathbb{A}_{\mathbb{Q}}^{n}$, the above result gives the bounds

$$
\operatorname{deg}_{y_{i}}(E) \leq \prod_{j \neq i} d_{j}, \quad \mathrm{~m}(E) \leq \sum_{\ell=1}^{n+1}\left(\prod_{j \neq \ell} d_{j}\right) h_{\ell}
$$

for the degree and the Mahler measure of $\overline{\operatorname{Im}(\psi)}$. Using Lemma 2.32(2), we can bound the height of this polynomial by

$$
\mathrm{h}(E) \leq \sum_{\ell=1}^{n+1}\left(\prod_{j \neq \ell} d_{j}\right)\left(h_{\ell}+\log (2)\right) .
$$

ExAmple 3.26. - Let $d_{1}, d_{2}, H_{1}, H_{2} \geq 1$ such that $d_{1}, d_{2}$ are coprime and set

$$
\frac{q_{1}}{p_{1}}=\frac{x^{d_{1}}}{H_{1}(x+1)^{d_{1}}}, \quad \frac{q_{2}}{p_{2}}=\frac{(x+1)^{d_{2}}}{H_{2} x^{d_{2}}} .
$$

The implicit equation of the closure of the image of

$$
\mathbb{A}_{\mathbb{Q}}^{1}-\rightarrow \mathbb{A}_{\mathbb{Q}}^{2}, \quad x \longmapsto\left(\frac{q_{1}}{p_{1}}(x), \frac{q_{2}}{p_{2}}(x)\right)
$$

is defined by $E=H_{1}{ }^{{ }_{2}} H_{2}{ }^{d_{1}} y_{1}^{d_{2}} y_{2}^{d_{1}}-1 \in \mathbb{Z}\left[y_{1}, y_{2}\right]$. We have $\max \left\{\operatorname{deg}\left(p_{j}\right), \operatorname{deg}\left(q_{j}\right)\right\}=d_{j}$ while $\log \left(\left\|q_{1}\right\|_{1}+\left\|p_{1}\right\|_{1}\right)=\log \left(1+H_{1} 2^{d_{1}}\right)$ and $\log \left(\left\|q_{2}\right\|_{1}+\left\|p_{2}\right\|_{1}\right)=\log \left(2^{d_{2}}+H_{2}\right)$. Hence, Theorem 3.24 gives

$$
\mathrm{m}(E) \leq d_{2} \log \left(1+H_{1} 2^{d_{1}}\right)+d_{1} \log \left(2^{d_{2}}+H_{2}\right) .
$$

Indeed, $\mathrm{m}(E)=d_{1} \log \left(H_{2}\right)+d_{2} \log \left(H_{1}\right)$ and so the obtained bound is optimal up a term of size $O\left(d_{1} d_{2}\right)$.

\section{Arithmetic Nullstellensätze}

\subsection{An effective approach to the Nullstellensatz}

Recall the statement of the weak Nullstellensatz over a variety:

Let $V \subset \mathbb{A}_{K}^{n}$ be a $K$-variety and $f_{1}, \ldots, f_{s} \in K\left[x_{1}, \ldots, x_{n}\right]$ without common zeros in $V(\bar{K})$. Then there exist $g_{1}, \ldots, g_{s} \in K\left[x_{1}, \ldots, x_{n}\right]$ such that $1=g_{1} f_{1}+\cdots+g_{s} f_{s}$ on $V$. 
We recall Jelonek's approach in [14] to produce bounds for the degree of the $g_{i}$ 's and explain how it can be adapted to obtain bounds for their height. Set $r=\operatorname{dim}(V)$. We assume without loss of generality that $s \leq r+1$, otherwise we reduce to this case by taking generic linear combinations of the input polynomials. Consider the regular map

$$
\varphi: V \times \mathbb{A}_{K}^{1} \longrightarrow V \times \mathbb{A}_{K}^{s}, \quad(\boldsymbol{x}, z) \longmapsto\left(\boldsymbol{x}, z f_{1}(\boldsymbol{x}), \ldots, z f_{s}(\boldsymbol{x})\right) .
$$

Since the $f_{j}$ 's have no common zeros in $V(\bar{K})$, the subsets $\left\{\left(\boldsymbol{x}, z_{1}, \ldots, z_{s}\right): f_{j}(\boldsymbol{x}) \neq 0\right\}$ form an open covering of $V \times \mathbb{A}_{K}^{s}$. This implies that

$$
\operatorname{Im}(\varphi)=\left\{\left(\boldsymbol{x}, z_{1}, \ldots, z_{s}\right) \in V \times \mathbb{A}_{K}^{s}: f_{i}(\boldsymbol{x}) z_{j}=f_{j}(\boldsymbol{x}) z_{i} \text { for all } i, j\right\} .
$$

In particular, $\operatorname{Im}(\varphi)$ is a subvariety of $V \times \mathbb{A}_{K}^{s}$. Furthermore, let $g_{1}, \ldots, g_{s}$ be polynomials such that $1=g_{1} f_{1}+\cdots+g_{s} f_{s}$. The map $\operatorname{Im}(\varphi) \rightarrow V \times \mathbb{A}_{K}^{1}$ defined as $\left(\boldsymbol{x}, z_{1}, \ldots, z_{s}\right) \mapsto$ $\left(\boldsymbol{x}, g_{1}(\boldsymbol{x}) z_{1}+\cdots+g_{s}(\boldsymbol{x}) z_{s}\right)$ is a left inverse of $\varphi$. Hence, $\varphi$ induces an isomorphism $V \times \mathbb{A}_{K}^{1} \rightarrow \operatorname{Im}(\varphi)$.

Assume there exists a finite linear projection $\pi: \operatorname{Im}(\varphi) \rightarrow \mathbb{A}_{K}^{r+1}$. Such a map exists if the field $K$ is sufficiently big (for instance, if it is infinite). In this case, we can assume without loss of generality that $\pi$ is given by a $(r+1) \times(n+s)$-matrix in reduced triangular form. Hence, there are linear forms $\ell_{i}=\gamma_{i, 1} x_{1}+\cdots+\gamma_{i, n} x_{n} \in K[\boldsymbol{x}], 1 \leq i \leq r+1$, such that this projection writes down as

$$
\pi: \operatorname{Im}(\varphi) \rightarrow \mathbb{A}_{K}^{r+1},\left(\boldsymbol{x}, z_{1}, \ldots, z_{s}\right) \mapsto\left(z_{1}+\ell_{1}(\boldsymbol{x}), \ldots, z_{s}+\ell_{s}(\boldsymbol{x}), \ell_{s+1}(\boldsymbol{x}), \ldots, \ell_{r+1}(\boldsymbol{x})\right) .
$$

Thus, the composition $\pi \circ \varphi: V \times \mathbb{A}_{K}^{1} \rightarrow \mathbb{A}_{K}^{r+1}$ is a finite map or equivalently, the inclusion of algebras $(\pi \circ \varphi)^{*}: K\left[y_{1}, \ldots, y_{r+1}\right] \hookrightarrow K[\boldsymbol{x}, z] / I\left(V \times \mathbb{A}_{K}^{1}\right)$ is an integral extension. This map writes down, for $(\boldsymbol{x}, z) \in V \times \mathbb{A}_{K}^{1}$, as

$$
\pi \circ \varphi(\boldsymbol{x}, z)=\left(z f_{1}(\boldsymbol{x})+\ell_{1}(\boldsymbol{x}), \ldots, z f_{s}(\boldsymbol{x})+\ell_{s}(\boldsymbol{x}), \ell_{s+1}(\boldsymbol{x}), \ldots, \ell_{r+1}(\boldsymbol{x})\right) .
$$

Up to a non-zero scalar in $K$, the minimal polynomial of $z$ over $K[\boldsymbol{y}]$ is of the form

$$
E=z^{\delta}+\sum_{j=1}^{\delta} \sum_{\boldsymbol{a} \in \mathbb{N}^{r+1}} \alpha_{\boldsymbol{a}, j} \boldsymbol{y}^{\boldsymbol{a}} z^{\delta-j} \in K[\boldsymbol{y}, z] .
$$

Therefore,

$$
z^{\delta}+\sum_{j=1}^{\delta} \sum_{\boldsymbol{a}} \alpha_{\boldsymbol{a}, j}\left(z f_{1}+\ell_{1}\right)^{a_{1}} \cdots\left(z f_{s}+\ell_{s}\right)^{a_{s}} \ell_{s+1}^{a_{s+1}} \cdots \ell_{r+1}^{\alpha_{r+1}} z^{\delta-j}=0 \quad \text { on } V \times \mathbb{A}^{1}
$$

and so all the coefficients in the expansion of (4.2) with respect to the variable $z$ vanish identically on $V$. We derive a Bézout identity from the coefficient of $z^{\delta}$ as follows: for each $1 \leq j \leq \delta$ and $\boldsymbol{a} \in \mathbb{N}^{r+1}$, the coefficient of $z^{j}$ in the expansion of

$$
\left(z f_{1}+\ell_{1}\right)^{a_{1}} \cdots\left(z f_{s}+\ell_{s}\right)^{a_{s}} \ell_{s+1}^{a_{s+1}} \cdots \ell_{r+1}^{\alpha_{r+1}}
$$

writes down as

$$
\sum_{\boldsymbol{b}} g_{\boldsymbol{b}} f_{1}^{b_{1}} \cdots f_{s}^{b_{s}}
$$

with $g_{\boldsymbol{b}} \in K[\boldsymbol{x}]$ and $\boldsymbol{b} \in \mathbb{N}^{s}$ such that $|\boldsymbol{b}|=j \geq 1$. Hence each term in this sum is a multiple of some $f_{j}$ and, regrouping terms, we can write the coefficient of $z^{\delta}$ in the expansion of (4.2) as $1-g_{1} f_{1}-\cdots-g_{s} f_{s}$ with $g_{i} \in K[\boldsymbol{x}]$. 
Remark 4.3. - The argument above is not a proof of the Nullstellensatz since it relies on the a priori existence of a Bézout identity: this is used to prove that the map $\varphi$ in (4.1) is an isomorphism onto its image.

In our treatment of the arithmetic Nullstellensatz, we will use the previous construction for the general linear forms

$$
\ell_{i}:=u_{i, 1} x_{1}+\cdots+u_{i, n} x_{n} \in K\left[\boldsymbol{u}_{i}\right][\boldsymbol{x}] \quad \text { for } 1 \leq i \leq r+1,
$$

where $\boldsymbol{u}_{i}=\left\{u_{i, j}\right\}_{1 \leq j \leq n}$ is a group of auxiliary variables. This is a valid choice, since the associated linear projection $\pi: \operatorname{Im}(\varphi)_{K(\boldsymbol{u})} \rightarrow \mathbb{A}_{K(\boldsymbol{u})}^{r+1}$ is a finite map, see for instance [21, Ch. 7, Thm. 2.2].

Assume that $K$ is the field of fractions of a factorial ring $A$. Let $E \in A[\boldsymbol{u}][\boldsymbol{y}, z]$ be the minimal polynomial of $z$ with respect to the map $\pi \circ \varphi$, primitive with respect to the ring $A[\boldsymbol{u}]$. We expand it as

$$
E=\alpha_{\mathbf{0}, 0} z^{\delta}+\sum_{j=1}^{\delta} \sum_{\boldsymbol{a} \in \mathbb{N}^{r+1}} \alpha_{\boldsymbol{a}, j} \boldsymbol{y}^{\boldsymbol{a}} z^{\delta-j}
$$

with $\alpha_{\boldsymbol{a}, j} \in A[\boldsymbol{u}]$ such that $\alpha_{\mathbf{0}, 0} \neq 0$. For $1 \leq i \leq s$, set

$$
\widetilde{g}_{i}=-\sum_{j=1}^{\delta} \sum_{\boldsymbol{a}} \sum_{\boldsymbol{b}} \alpha_{\boldsymbol{a}, j}\left(\left(\prod_{k=1}^{r+1}\left(\begin{array}{l}
a_{k} \\
b_{k}
\end{array}\right) \ell_{k}^{a_{k}-b_{k}}\right) f_{1}^{b_{1}} \cdots f_{i-1}^{b_{i-1}} f_{i}^{b_{i}-1}\right) \in A[\boldsymbol{u}][\boldsymbol{x}],
$$

the sums being indexed by all $\boldsymbol{a}, \boldsymbol{b} \in \mathbb{N}^{r+1}$ such that $(\boldsymbol{a}, j) \in \operatorname{supp}(E)$ and $|\boldsymbol{b}|=j, b_{k} \leq a_{k}$ for $1 \leq k \leq i, b_{k}=0$ for $i+1 \leq k \leq r+1$ and $b_{i} \geq 1$. Using (4.2), we can verify that $\alpha_{\mathbf{0}, 0}-\widetilde{g}_{1} f_{1}-\cdots-\widetilde{g}_{s} f_{s}$ is the coefficient of $z^{\delta}$ in the expansion of

$$
E\left(z f_{1}+\ell_{1}, \ldots, z f_{s}+\ell_{s}, \ell_{s+1}, \ldots, \ell_{r+1}, z\right) .
$$

Hence,

$$
\alpha_{\mathbf{0}, 0}=\widetilde{g}_{1} f_{1}+\cdots+\widetilde{g}_{s} f_{s} \quad \text { on } V_{K(\boldsymbol{u})} .
$$

We then extract a Bézout identity on $V$ by considering the coefficient of any monomial in $u$ in (4.7) appearing in the monomial expansion of $\alpha_{\mathbf{0}, 0}$.

We need the following lemma relating minimal polynomials with the implicitization problem.

Lemma 4.8. - Let $V \subset \mathbb{A}_{K}^{n}$ be a $K$-variety of pure dimension $r$ and $q_{1}, \ldots, q_{r+1} \in K[\boldsymbol{x}, z]$ such that the map

$$
V \times \mathbb{A}_{K}^{1} \longrightarrow \mathbb{A}_{K}^{r+1}, \quad(\boldsymbol{x}, z) \longmapsto\left(q_{1}(\boldsymbol{x}, z), \ldots, q_{r+1}(\boldsymbol{x}, z)\right)
$$

is finite. Let $E \in K[\boldsymbol{y}][z] \backslash\{0\}$ be the minimal polynomial of $z$ with respect to this map. Then the map

$$
\psi: V_{K(z)} \longrightarrow \mathbb{A}_{K(z)}^{r+1}, \quad \boldsymbol{x} \longmapsto\left(q_{1}(\boldsymbol{x}), \ldots, q_{r+1}(\boldsymbol{x})\right)
$$

is generically finite onto its image. Furthermore, $E \in K[z][\boldsymbol{y}]$ is a squarefree polynomial, primitive with respect to the ring $K[z]$, defining $\overline{\operatorname{Im}(\Psi)}$. 
Proof. - We reduce without loss of generality to the case of an irreducible $K$-variety $V$. Consider the map

$$
\rho: V \times \mathbb{A}_{K}^{1} \longrightarrow \mathbb{A}_{K}^{r+1} \times \mathbb{A}_{K}^{1}, \quad(\boldsymbol{x}, z) \longmapsto\left(q_{1}(\boldsymbol{x}, z), \ldots, q_{r+1}(\boldsymbol{x}, z), z\right) .
$$

Its image is the irreducible $K$-hypersurface defined by $E$. In algebraic terms, the kernel of $\rho^{*}: K[\boldsymbol{y}, z] \rightarrow K[\boldsymbol{x}, z] / I\left(V \times \mathbb{A}_{K}^{1}\right)$ is the principal ideal generated by $E$. Furthermore, $E \notin K[z]$ as, otherwise, this would imply that the $q_{j}$ 's are constant on $V \times \mathbb{A}_{K}^{1}$, which is not possible because of the finiteness assumption.

We have $K[\boldsymbol{x}, z] / I\left(V \times \mathbb{A}_{K}^{1}\right)=K[z] \otimes_{K} K[\boldsymbol{x}] / I(V)$. Hence, $E$ is also a generator of the kernel of $\psi^{*}: K(z)[\boldsymbol{y}] \rightarrow K(z)[\boldsymbol{x}] / I\left(V_{K(z)}\right)$. The image of $\psi$ is a hypersurface of $\mathbb{A}_{K(z)}^{r+1}$, since $E$ does not lie in $K[z]$. Hence, this map is generically finite onto its image. Furthermore, $E$ is an irreducible polynomial defining this hypersurface, primitive with respect to the ring $K[z]$.

Consider the polynomials $q_{j} \in A[\boldsymbol{u}][\boldsymbol{x}, z]$ defined by

$$
q_{j}= \begin{cases}z f_{j}+\ell_{j}, & \text { for } 1 \leq j \leq s, \\ \ell_{j}, & \text { for } s+1 \leq j \leq r+1 .\end{cases}
$$

In our setting, $\pi \circ \varphi: V_{K(\boldsymbol{u})} \times \mathbb{A}_{K(\boldsymbol{u})}^{1} \longrightarrow \mathbb{A}_{K(\boldsymbol{u})}^{r+1}$ is finite. Hence, Lemma 4.8 implies that the polynomial $E$ in (4.5) is a squarefree polynomial, primitive with respect to the $\operatorname{ring} A[\boldsymbol{u}, z]$, defining the closure of the image of the generically finite map

$$
\psi: V_{K(\boldsymbol{u}, z)} \longrightarrow \mathbb{A}_{K(\boldsymbol{u}, z)}^{r+1}, \quad \boldsymbol{x} \longmapsto\left(q_{1}(\boldsymbol{x}), \ldots, q_{r+1}(\boldsymbol{x})\right) .
$$

Thus, we can produce bounds for its size by using a suitable version of Perron's theorem. In turn, this will allow us to bound the size of the polynomials in the associated Bézout identity. We will see the details in the next sections.

\subsection{Parametric Nullstellensätze}

We now apply the previous construction together with the parametric Perron's theorem to produce different Nullstellensätze for polynomials with coefficients depending on groups of parameters. Let $k$ be a field. Consider groups of variables $\boldsymbol{x}=\left\{x_{1}, \ldots, x_{n}\right\}$ and $\boldsymbol{t}_{l}=\left\{t_{l, 1}, \ldots, t_{l, p_{l}}\right\}, 1 \leq l \leq m$. Set $\boldsymbol{t}=\left\{\boldsymbol{t}_{1}, \ldots, \boldsymbol{t}_{m}\right\}$ and, for each $1 \leq l \leq m$, write $k_{l}=k\left(\boldsymbol{t}_{1}, \ldots, \boldsymbol{t}_{l-1}, \boldsymbol{t}_{l+1}, \ldots, \boldsymbol{t}_{m}\right)$. Recall that $k_{l}\left(\boldsymbol{t}_{l}\right)=k(\boldsymbol{t})$ and that for a projective $k(\boldsymbol{t})$-variety $V$, we denote by $\mathrm{h}_{\boldsymbol{t}_{l}}(V)$ its height with respect to the base ring $k_{l}\left[\boldsymbol{t}_{l}\right]$.

Theorem 4.11. - Let $V \subset \mathbb{A}_{k(t)}^{n}$ be a $k(\boldsymbol{t})$-variety of pure dimension $r$ and $f_{1}, \ldots, f_{s} \in k[\boldsymbol{t}, \boldsymbol{x}] \backslash k[\boldsymbol{t}]$ a family of $s \leq r+1$ polynomials without common zeros in $V(\overline{k(\boldsymbol{t})})$. Set $d_{j}=\operatorname{deg}_{\boldsymbol{x}}\left(f_{j}\right)$ and $h_{l, j}=\operatorname{deg}_{\boldsymbol{t}_{l}}\left(f_{j}\right)$ for $1 \leq j \leq s$ and $1 \leq l \leq m$. Then there exist $\alpha \in k[\boldsymbol{t}] \backslash\{0\}$ and $g_{1}, \ldots, g_{s} \in k[\boldsymbol{t}, \boldsymbol{x}]$ such that

$$
\alpha=g_{1} f_{1}+\cdots+g_{s} f_{s} \quad \text { on } V
$$


with

$$
\begin{aligned}
& \text { - } \operatorname{deg}_{\boldsymbol{x}}\left(g_{i} f_{i}\right) \leq\left(\prod_{j=1}^{s} d_{j}\right) \operatorname{deg}(V), \\
& \text { - } \operatorname{deg}_{\boldsymbol{t}_{l}}(\alpha), \operatorname{deg}_{\boldsymbol{t}_{l}}\left(g_{i} f_{i}\right) \leq\left(\prod_{j=1}^{s} d_{j}\right)\left(\mathrm{h}_{\boldsymbol{t}_{l}}(V)+\operatorname{deg}(V) \sum_{\ell=1}^{s} \frac{h_{l, \ell}}{d_{\ell}}\right) \quad \text { for } 1 \leq l \leq m .
\end{aligned}
$$

Proof. - We apply the construction explained in $\S 4.1$ to the $\operatorname{ring} A:=k[\boldsymbol{t}]$, the variety $V$ and the polynomials $f_{j}$. We will freely use the notations introduced in that section. As a result of that construction, we obtain $\alpha \in A \backslash\{0\}$ and $g_{i} \in A[\boldsymbol{x}]$ satisfying the Bézout identity (4.12). These scalar and polynomials are obtained as the coefficients of a monomial in the variables $\boldsymbol{u}$ in the Bézout identity on $V_{K(\boldsymbol{u})}$ in (4.7). Hence,

$$
\operatorname{deg}_{\boldsymbol{x}}\left(g_{i} f_{i}\right) \leq \operatorname{deg}_{\boldsymbol{x}}\left(\widetilde{g}_{i} f_{i}\right), \operatorname{deg}_{\boldsymbol{t}_{l}}(\alpha) \leq \operatorname{deg}_{\boldsymbol{t}_{l}}\left(\alpha_{\mathbf{0}, 0}\right), \operatorname{deg}_{\boldsymbol{t}_{l}}\left(g_{i} f_{i}\right) \leq \operatorname{deg}_{\boldsymbol{t}_{l}}\left(\widetilde{g}_{i} f_{i}\right) .
$$

Let $E \in A[\boldsymbol{u}][\boldsymbol{y}, z]$ be the polynomial in (4.5) and set $\boldsymbol{d}=\left(d_{1}, \ldots, d_{s}, 1, \ldots, 1\right) \in \mathbb{N}^{r+1}$. From the definition of $\widetilde{g}_{i}$ in (4.6) and using that, by hypothesis, $d_{i} \geq 1$ for all $i$,

$$
\begin{array}{r}
\operatorname{deg}_{\boldsymbol{x}}\left(\widetilde{g}_{i} f_{i}\right) \leq \max _{j, \boldsymbol{a}, \boldsymbol{b}}\left\{\operatorname{deg}_{\boldsymbol{x}}\left(\alpha_{\boldsymbol{a}, j}\left(\prod_{e=1}^{r+1}\left(\begin{array}{c}
a_{e} \\
b_{e}
\end{array}\right) \ell_{e}^{a_{e}-b_{e}}\right) f_{1}^{b_{1}} \cdots f_{i-1}^{b_{i-1}} f_{i}^{b_{i}}\right)\right\} \\
\leq \max _{\boldsymbol{a}, \boldsymbol{b}}\left\{\sum_{e=1}^{r+1}\left(a_{e}-b_{e}\right)+\sum_{\ell=1}^{i} d_{\ell} b_{\ell}\right\} \leq \max _{\boldsymbol{a}}\langle\boldsymbol{d}, \boldsymbol{a}\rangle
\end{array}
$$

with $1 \leq j \leq \operatorname{deg}_{z}(E), \boldsymbol{a} \in \mathbb{N}^{r+1}$ in the support of $E$ with respect to the variables $\boldsymbol{y}$ and $\boldsymbol{b} \in \mathbb{N}^{r+1}$ satisfying $|\boldsymbol{b}|=j, b_{e} \leq a_{e}$ for $1 \leq e \leq i, b_{e}=0$ for $i+1 \leq e \leq r+1$ and $b_{i} \geq 1$. For $1 \leq l \leq m$ we set $\boldsymbol{h}_{l}=\left(h_{l, 1}, \ldots, h_{l, s}, 0, \ldots, 0\right) \in \mathbb{N}^{r+1}$. Using Lemma 2.1, we obtain similarly

$$
\begin{aligned}
\operatorname{deg}_{\boldsymbol{t}_{l}}\left(\widetilde{g}_{i} f_{i}\right) & \leq \max _{j, \boldsymbol{a}, \boldsymbol{b}}\left\{\operatorname{deg}_{\boldsymbol{t}_{l}}\left(\alpha_{\boldsymbol{a}, j}\left(\prod_{e=1}^{r+1}\left(\begin{array}{c}
a_{e} \\
b_{e}
\end{array}\right) \ell_{e}^{a_{e}-b_{e}}\right) f_{1}^{b_{1}} \cdots f_{i-1}^{b_{i-1}} f_{i}^{b_{i}}\right)\right\} \\
& \leq \max _{j, \boldsymbol{a}, \boldsymbol{b}}\left\{\operatorname{deg}_{\boldsymbol{t}_{l}}\left(\alpha_{\boldsymbol{a}, j}\right)+\sum_{\ell=1}^{i} h_{l, \ell} b_{\ell}\right\} \\
& \leq \max _{\boldsymbol{a}}\left\{\operatorname{deg}_{\boldsymbol{t}_{l}}\left(\alpha_{\boldsymbol{a}}\right)+\left\langle\boldsymbol{h}_{l}, \boldsymbol{a}\right\rangle\right\} .
\end{aligned}
$$

By Lemma 4.8, $E$ is a primitive and squarefree polynomial in $k[\boldsymbol{t}, \boldsymbol{u}, z][\boldsymbol{y}]$ defining the closure of the image of the map $\psi$ in (4.10). Hence, we can apply Corollary 3.14 to bound the partial degrees of this polynomial. We have $\operatorname{deg}_{\boldsymbol{x}}\left(q_{j}\right)=d_{j}, \operatorname{deg}_{\boldsymbol{t}_{l}}\left(q_{j}\right)=h_{l, j}$ for $1 \leq j \leq s$ while $\operatorname{deg}_{\boldsymbol{x}}\left(q_{j}\right)=1, \operatorname{deg}_{\boldsymbol{t}_{l}}\left(q_{j}\right)=0$ for $s+1 \leq j \leq r+1$. By Proposition 2.13(2), $\operatorname{deg}\left(V_{k(\boldsymbol{t}, \boldsymbol{u}, z)}\right)=\operatorname{deg}(V)$ and $\mathrm{h}_{\boldsymbol{t}_{l}}\left(V_{k(\boldsymbol{t}, \boldsymbol{u}, z)}\right)=\mathrm{h}_{\boldsymbol{t}_{l}}(V)$. Therefore, for all $\boldsymbol{a} \in \operatorname{supp}(E)$,

$$
\langle\boldsymbol{d}, \boldsymbol{a}\rangle \leq\left(\prod_{j=1}^{s} d_{j}\right) \operatorname{deg}(V), \operatorname{deg}_{\boldsymbol{t}_{l}}\left(\alpha_{\boldsymbol{a}}\right)+\left\langle\boldsymbol{h}_{l}, \boldsymbol{a}\right\rangle \leq\left(\prod_{j=1}^{s} d_{j}\right)\left(\mathrm{h}_{\boldsymbol{t}_{l}}(V)+\operatorname{deg}(V) \sum_{\ell=1}^{s} \frac{h_{l, \ell}}{d_{\ell}}\right) .
$$

The statement follows from this inequality together with (4.13), (4.14) and (4.15).

Theorem 5 in the introduction corresponds to the case of polynomials depending on one group of parameters. It follows readily from the above result and the fact that a variety defined over $k(\boldsymbol{t})$ can be identified with a $k(\boldsymbol{t})$-variety, see Remark 1.1. 
Example 4.16. - Consider the following variant of a classical example due to Masser and Philippon: let $t$ be a variable, $d_{1}, \ldots, d_{n} \geq 1, h \geq 0$ and set

$$
\begin{aligned}
f_{1}=x_{1}^{d_{1}}, f_{2}=x_{1} x_{n}^{d_{2}-1}-x_{2}^{d_{2}}, \ldots, f_{n-1}=x_{n-2} x_{n-1}^{d_{n-1}-1}-x_{n-1}^{d_{n-1}}, \\
f_{n}=x_{n-1} x_{n}^{d_{n}-1}-t^{h} \in k\left[t, x_{1}, \ldots, x_{n}\right] .
\end{aligned}
$$

It is a system of $n$ polynomials without common zeros in $\overline{k(t)}^{n}$. We have $\operatorname{deg}_{\boldsymbol{x}}\left(f_{j}\right)=d_{j}$ for all $j$ while $\operatorname{deg}_{t}\left(f_{j}\right)=0$ for $1 \leq j \leq n-1$ and $\operatorname{deg}_{t}\left(f_{n}\right)=h$. Theorem 4.11 implies that there exists a Bézout identity $\alpha=g_{1} f_{1}+\cdots+g_{n} f_{n}$ with

$$
\operatorname{deg}_{\boldsymbol{x}}\left(g_{i} f_{i}\right) \leq d_{1} \cdots d_{n}, \quad \operatorname{deg}(\alpha), \operatorname{deg}_{t}\left(g_{i} f_{i}\right) \leq d_{1} \cdots d_{n-1} h .
$$

To obtain a lower bound, consider a further variable $u$ and specialize any such Bézout identity at $x_{i}=\gamma_{i}$ with

$$
\gamma_{1}=t^{d_{2} \cdots d_{n-1} h} u^{d_{2} \cdots d_{n}-1}, \ldots, \gamma_{n-2}=t^{d_{n-1} h} u^{d_{n-1} d_{n}-1}, \gamma_{n-1}=t^{h} u^{d_{n}-1}, \gamma_{n}=1 / u .
$$

We obtain that $\alpha=g_{1}\left(\gamma_{1}, \ldots, \gamma_{n-1}, 1 / u\right) t^{d_{1} \cdots d_{n-1} h} u^{d_{1} \cdots d_{n}-d_{1}}$. From this, we deduce the lower bounds

$$
\operatorname{deg}_{\boldsymbol{x}}\left(g_{1} f_{1}\right) \geq d_{1} \cdots d_{n}, \quad \operatorname{deg}(\alpha) \geq d_{1} \cdots d_{n-1} h .
$$

Hence, both bounds in (4.17) are optimal in this case.

ExAmple 4.18. - Let $V \subset \mathbb{A}_{k}^{n}$ be a $k$-variety of pure dimension $r$. For $1 \leq j \leq r+1$, let $d_{j} \geq 1$ and consider the general $n$-variate polynomial of degree $d_{j}$

$$
F_{j}=\sum_{|\boldsymbol{a}| \leq d_{j}} u_{j, \boldsymbol{a}} \boldsymbol{x}^{\boldsymbol{a}} \in k\left[\boldsymbol{u}_{j}\right]\left[x_{1}, \ldots, x_{n}\right]
$$

where $\boldsymbol{u}_{j}=\left\{u_{j, \boldsymbol{a}}\right\}_{|\boldsymbol{a}| \leq d_{j}}$. Set $\boldsymbol{u}=\left\{\boldsymbol{u}_{1}, \ldots, \boldsymbol{u}_{r+1}\right\}$. It is not difficult to verify that the $F_{j}$ 's have no common zeros in $V_{k(\boldsymbol{u})}(\overline{k(\boldsymbol{u})})$. Hence, we can apply Theorem 4.11 to the $F_{j}$ 's as a system of polynomials with coefficients depending on the groups of parameters $\boldsymbol{u}_{1}, \ldots, \boldsymbol{u}_{r+1}$. We have that $\operatorname{deg}\left(V_{k(\boldsymbol{u})}\right)=\operatorname{deg}(V)$ and, by Proposition 2.13(2), $\mathrm{h}_{\boldsymbol{u}_{l}}\left(V_{k(\boldsymbol{u})}\right)=0$. Besides, $\operatorname{deg}_{\boldsymbol{x}}\left(F_{j}\right)=d_{j}$ and $\operatorname{deg}_{\boldsymbol{u}_{l}}\left(F_{j}\right)$ is equal to 1 if $l=j$ and to 0 otherwise. We deduce that there exist $\alpha \in k[\boldsymbol{u}] \backslash\{0\}$ and $g_{i} \in k[\boldsymbol{u}]\left[x_{1}, \ldots, x_{n}\right]$ such that $\alpha=g_{1} F_{1}+\cdots+g_{r+1} F_{r+1}$ on $V_{k(u)}$ with

$$
\operatorname{deg}_{\boldsymbol{x}}\left(g_{i} F_{i}\right) \leq\left(\prod_{j=1}^{r+1} d_{j}\right) \operatorname{deg}(V), \quad \operatorname{deg}_{\boldsymbol{u}_{l}}(\alpha), \operatorname{deg}_{\boldsymbol{u}_{l}}\left(g_{i} F_{i}\right) \leq\left(\prod_{j \neq l} d_{j}\right) \operatorname{deg}(V) .
$$

Using Lemma 1.34 and Corollary 1.33, we can verify that the elimination ideal

$$
\left(I(V) k[\boldsymbol{u}]\left[x_{1}, \ldots, x_{n}\right]+\left(F_{1}, \ldots, F_{r+1}\right)\right) \cap k[\boldsymbol{u}]
$$

is generated by the resultant $\operatorname{Res}_{d_{1}, \ldots, d_{r+1}}(\bar{V})$ of the closure of $V$ in $\mathbb{P}_{k}^{n}$. This is a multihomogeneous polynomial of partial degrees

$$
\operatorname{deg}_{\boldsymbol{u}_{l}}\left(\operatorname{Res}_{d_{1}, \ldots, d_{r+1}}(\bar{V})\right)=\left(\prod_{j \neq l} d_{j}\right) \operatorname{deg}(V) .
$$

Hence, $\alpha$ is a multiple of this resultant and, comparing degrees, we see that $\alpha$ and $\operatorname{Res}_{d_{1}, \ldots, d_{r+1}}(\bar{V})$ coincide up to a factor in $k^{\times}$. This implies that the bound for the $\boldsymbol{u}_{l}$-degrees in (4.19) is optimal. Observe that the bound for the $\boldsymbol{x}$-degree is not optimal, at least when 
$V=\mathbb{A}_{k}^{n}$. In this case, it can be shown that there exist $g_{i}$ 's satisfying the same bound for the $\boldsymbol{u}_{l}$-degree and such that $\operatorname{deg}_{\boldsymbol{x}}\left(g_{i} f_{i}\right) \leq\left(\sum_{j=1}^{n+1} d_{j}\right)-n$.

The following result is a partial extension of Theorem 4.11 to an arbitrary number of polynomials. For simplicity, we only state it for polynomials depending on one group of parameters $\boldsymbol{t}=\left\{t_{1}, \ldots, t_{p}\right\}$. In this setting, we loose track of the contribution of the $\boldsymbol{t}$-degrees of most of the individual input polynomials. However, it is possible to differentiate the contribution of one of them, which will be important in the proof of the strong parametric Nullstellensatz.

COROllary 4.20. - Let $V \subset \mathbb{A}_{k(t)}^{n}$ be a $k(\boldsymbol{t})$-variety of pure dimension $r$ and $f_{1}, \ldots, f_{s} \in k[\boldsymbol{t}, \boldsymbol{x}] \backslash k[\boldsymbol{t}]$ without common zeros in $V(\overline{k(\boldsymbol{t})})$ such that $V\left(f_{s}\right)$ intersects $V$ properly. Set $d_{j}=\operatorname{deg}_{\boldsymbol{x}}\left(f_{j}\right)$ for $1 \leq j \leq s$. Assume that $d_{1} \geq \cdots \geq d_{s-1}$ and that $d_{s}$ is arbitrary. Set also $h=\max _{1 \leq j \leq s-1} \operatorname{deg}_{\boldsymbol{t}}\left(f_{j}\right)$ and $h_{s}=\operatorname{deg}_{\boldsymbol{t}}\left(f_{s}\right)$. Then there exist $\alpha \in k[\boldsymbol{t}] \backslash\{0\}$ and $g_{1}, \ldots, g_{s} \in k[\boldsymbol{t}, \boldsymbol{x}]$ such that $\alpha=g_{1} f_{1}+\cdots+g_{s} f_{s}$ on $V$ with

$$
\begin{aligned}
& \text { - } \operatorname{deg}_{\boldsymbol{x}}\left(g_{i} f_{i}\right) \leq\left(d_{s} \prod_{j=1}^{\min \{s-1, r\}} d_{j}\right) \operatorname{deg}(V), \\
& \text { - } \operatorname{deg}(\alpha), \operatorname{deg}_{\boldsymbol{t}}\left(g_{i} f_{i}\right) \leq\left(d_{s} \prod_{j=1}^{\min \{s-1, r\}} d_{j}\right)\left(\mathrm{h}(V)+\operatorname{deg}(V)\left(\frac{h_{s}}{d_{s}}+\sum_{\ell=1}^{\min \{s-1, r\}} \frac{h}{d_{\ell}}\right)\right) .
\end{aligned}
$$

Proof. - If $s \leq r+1$, the result follows from the case $m=1$ in Theorem 4.11. In the case $s \geq r+2$, we can reduce to $r+1$ polynomials by taking linear combinations as follows: let $\boldsymbol{v}=\left\{v_{j, i}\right\}_{1 \leq j \leq r, 1 \leq i \leq s-r-1}$ be a group of variables and set $K=k(\boldsymbol{v})$. Write

$$
\bar{f}_{j}=f_{j}+v_{j, 1} f_{r+1}+\cdots+v_{j, s-r-1} f_{s-1} \quad \text { for } 1 \leq j \leq r, \quad \bar{f}_{r+1}=f_{s} .
$$

The hypothesis that $V\left(f_{s}\right)$ and $V$ intersect properly implies that the polynomials above do not have common zeros in $V_{K(\boldsymbol{t})}(\overline{K(\boldsymbol{t})})$. We have that $\operatorname{deg}_{\boldsymbol{x}}\left(\bar{f}_{j}\right)=d_{j}$ and $\operatorname{deg}_{\boldsymbol{t}}\left(\bar{f}_{j}\right)=h$ for $1 \leq j \leq r$ while $\operatorname{deg}_{\boldsymbol{x}}\left(\bar{f}_{r+1}\right)=d_{s}$ and $\operatorname{deg}_{\boldsymbol{t}}\left(\bar{f}_{r+1}\right)=h_{s}$. By Theorem 4.11, there exist $\bar{\alpha} \in k[\boldsymbol{v}, \boldsymbol{t}] \backslash\{0\}$ and $\bar{g}_{i} \in k[\boldsymbol{v}, \boldsymbol{t}][\boldsymbol{x}]$ such that $\bar{\alpha}=\bar{g}_{1} \bar{f}_{1}+\cdots+\bar{g}_{r+1} \bar{f}_{r+1}$ on $V_{K(\boldsymbol{t})}$ with

$$
\begin{aligned}
& \operatorname{deg}_{\boldsymbol{x}}\left(\bar{g}_{i} \bar{f}_{i}\right) \leq\left(d_{s} \prod_{j=1}^{r} d_{j}\right) \operatorname{deg}(V), \\
& \operatorname{deg}_{\boldsymbol{t}}(\bar{\alpha}), \operatorname{deg}_{\boldsymbol{t}}\left(\bar{g}_{i} \bar{f}_{i}\right) \leq\left(d_{s} \prod_{j=1}^{r} d_{j}\right)\left(\mathrm{h}(V)+\operatorname{deg}(V)\left(\frac{h_{s}}{d_{s}}+\sum_{\ell=1}^{r} \frac{h}{d_{\ell}}\right)\right) .
\end{aligned}
$$

Unfolding the linear combinations in the above identity and taking a non-zero coefficient with respect to the variables $\boldsymbol{v}$, we extract a Bézout identity $\alpha=g_{1} f_{1}+\cdots+g_{s} f_{s}$ on $V$ satisfying the same degree bounds.

REMARK 4.21. - The previous results by Smietanski are for polynomials depending on at most two parameters without common zeros in the affine space [35]. For instance, let $f_{1}, \ldots, f_{s}$ be polynomials in $k\left[t_{1}, t_{2}\right]\left[x_{1}, \ldots, x_{n}\right] \backslash k\left[t_{1}, t_{2}\right]$ without common zeros in $\mathbb{A}^{n}\left(\overline{k\left(t_{1}, t_{2}\right)}\right)$. Set $d_{j}=\operatorname{deg}\left(f_{j}\right), h=\max _{j} \operatorname{deg}_{t_{1}, t_{2}}\left(f_{j}\right)$, and suppose that 
$d_{2} \geq \cdots \geq d_{s} \geq d_{1}$. Set also $\nu=\min \{n+1, s\}$. In [35], it is shown that there exist $\alpha \in k\left[t_{1}, t_{2}\right] \backslash\{0\}$ and $g_{i} \in k\left[t_{1}, t_{2}\right]\left[x_{1}, \ldots, x_{n}\right]$ such that $\alpha=g_{1} f_{1}+\cdots+g_{s} f_{s}$ with

$$
\begin{aligned}
& \text { - } \operatorname{deg}_{\boldsymbol{x}}\left(g_{i} f_{i}\right) \leq 3 \prod_{j=1}^{\nu} d_{j}, \\
& \text { - } \operatorname{deg}_{t_{1}, t_{2}}(\alpha), \operatorname{deg}_{t_{1}, t_{2}}\left(g_{i} f_{i}\right) \leq \prod_{j=1}^{\nu}\left(d_{j}+h\right)+3^{\nu-1}\left(\prod_{j=1}^{\nu} d_{j}\right)\left(\sum_{\ell=1}^{\nu} \frac{1}{d_{\ell}}\right) h .
\end{aligned}
$$

We deduce from Corollary 4.20 the following parametric version of the strong effective Nullstellensatz.

Theorem 4.22. - Let $V \subset \mathbb{A}_{k(t)}^{n}$ be a $k(\boldsymbol{t})$-variety of pure dimension $r$ and $g, f_{1}, \ldots, f_{s} \in k[\boldsymbol{t}, \boldsymbol{x}]$ such that $g$ vanishes on the set of common zeros of $f_{1}, \ldots, f_{s}$ in $V(\overline{k(\boldsymbol{t})})$ and $\operatorname{deg}_{\boldsymbol{x}}\left(f_{1}\right) \geq \cdots \geq \operatorname{deg}_{\boldsymbol{x}}\left(f_{s}\right) \geq 1$. Set $d_{j}=\operatorname{deg}_{\boldsymbol{x}}\left(f_{j}\right)$ for $1 \leq j \leq s$, $h=\max _{1 \leq j \leq s} \operatorname{deg}_{\boldsymbol{t}}\left(f_{j}\right), d_{0}=\max \left\{1, \operatorname{deg}_{\boldsymbol{x}}(g)\right\}$ and $h_{0}=\operatorname{deg}_{\boldsymbol{t}}(g)$. Then there exist $\mu \in \mathbb{N}$, $\alpha \in k[\boldsymbol{t}] \backslash\{0\}$ and $g_{1}, \ldots, g_{s} \in k[\boldsymbol{t}, \boldsymbol{x}]$ such that

$$
\alpha g^{\mu}=g_{1} f_{1}+\cdots+g_{s} f_{s} \quad \text { on } V
$$

with

$$
\begin{aligned}
& \text { - } \mu \leq 2\left(\prod_{j=1}^{\min \{s, r+1\}} d_{j}\right) \operatorname{deg}(V), \\
& \text { - } \operatorname{deg}_{\boldsymbol{x}}\left(g_{i} f_{i}\right) \leq 4\left(\prod_{j=0}^{\min \{s, r+1\}} d_{j}\right) \operatorname{deg}(V), \\
& \text { - } \operatorname{deg}(\alpha), \operatorname{deg}_{\boldsymbol{t}}\left(g_{i} f_{i}\right) \leq 2\left(\prod_{j=0}^{\min \{s, r+1\}} d_{j}\right)\left(\mathrm{h}(V)+\operatorname{deg}(V)\left(\frac{3 h_{0}}{2 d_{0}}+\sum_{\ell=1}^{\min \{s, r+1\}} \frac{h}{d_{\ell}}\right)\right) .
\end{aligned}
$$

Proof. - Set $W=V \times \mathbb{A}_{k(t)}^{1}$, let $y$ be an additional variable and consider

$$
1-y^{d_{0}} g, f_{1}, \ldots, f_{s} \in k[\boldsymbol{t}, \boldsymbol{x}, y] .
$$

These polynomials have no common zeros on $W(\overline{k(\boldsymbol{t})})$ and $V\left(1-y^{d_{0}} g\right)$ intersects $W$ properly. We have that $\operatorname{dim}(W)=r+1, \operatorname{deg}(W)=\operatorname{deg}(V)$ and $\mathrm{h}(W)=\mathrm{h}(V)$, thanks to Lemma 3.2. Besides, $\operatorname{deg}_{\boldsymbol{x}, y}\left(f_{j}\right)=d_{j}$ and $\operatorname{deg}_{\boldsymbol{t}}\left(f_{j}\right) \leq h$ for $1 \leq j \leq s$, while $\operatorname{deg}_{\boldsymbol{x}, y}\left(1-y^{d_{0}} g\right)=2 d_{0}$ and $\operatorname{deg}_{\boldsymbol{t}}\left(1-y^{d_{0}} g\right)=h_{0}$. By Corollary 4.20, there exist $\alpha \in k[\boldsymbol{t}] \backslash\{0\}$ and $\bar{g}_{i} \in k[\boldsymbol{t}][\boldsymbol{x}, y]$ such that

$$
\alpha=\bar{g}_{0}\left(1-y^{d_{0}} g\right)+\bar{g}_{1} f_{1}+\cdots+\bar{g}_{s} f_{s} \quad \text { on } W
$$

with

$$
\begin{aligned}
& \operatorname{deg}_{\boldsymbol{x}, y}\left(\bar{g}_{i} f_{i}\right) \leq 2\left(\prod_{j=0}^{\min \{s, r+1\}} d_{j}\right) \operatorname{deg}(V), \\
& \operatorname{deg}(\alpha), \operatorname{deg}_{\boldsymbol{t}}\left(\bar{g}_{i} f_{i}\right) \leq 2\left(\prod_{j=0}^{\min \{s, r+1\}} d_{j}\right)\left(\mathrm{h}(V)+\operatorname{deg}(V)\left(\frac{h_{0}}{2 d_{0}}+\sum_{\ell=1}^{\min \{s, r+1\}} \frac{h}{d_{\ell}}\right)\right) .
\end{aligned}
$$


The input system (4.23) lies in the subring $k\left[\boldsymbol{t}, \boldsymbol{x}, y^{d_{0}}\right]$ of $k[\boldsymbol{t}, \boldsymbol{x}, y]$ and we can suppose without loss of generality that the Bézout identity (4.24) lies in this subring. Let $\widehat{g}_{i} \in k[\boldsymbol{t}, \boldsymbol{x}, y]$ such that $\bar{g}_{i}(\boldsymbol{t}, \boldsymbol{x}, y)=\widehat{g}_{i}\left(\boldsymbol{t}, \boldsymbol{x}, y^{d_{0}}\right)$. Then

$$
\alpha=\widehat{g}_{0}(1-y g)+\widehat{g}_{1} f_{1}+\cdots+\widehat{g}_{s} f_{s} .
$$

Specializing $y$ at $1 / g(\boldsymbol{t}, \boldsymbol{x})$ in the above identity and multiplying by a suitable denominator, we obtain an identity of the form $\alpha g^{\mu}=g_{1} f_{1}+\cdots+g_{s} f_{s}$ with $\mu=\max _{l} \operatorname{deg}_{y}\left(\widehat{g}_{l}\right)$ and $g_{i}=g^{\max _{l} \operatorname{deg}_{y}\left(\widehat{g}_{l}\right)} \widehat{g}_{i}(\boldsymbol{x}, 1 / g)$. Therefore,

$$
\mu=\max _{l} \operatorname{deg}_{y}\left(\widehat{g}_{l}\right) \leq \max _{l}\left\{\frac{\operatorname{deg}_{\boldsymbol{x}, y}\left(\bar{g}_{l}\right)}{d_{0}}\right\} \leq 2\left(\prod_{j=1}^{\min \{s, r+1\}} d_{j}\right) \operatorname{deg}(V) .
$$

Besides, $\operatorname{deg}_{\boldsymbol{x}, y}\left(\widehat{g}_{i} f_{i}\right) \leq \operatorname{deg}_{\boldsymbol{x}, y}\left(\bar{g}_{i} f_{i}\right)$ and $\operatorname{deg}_{\boldsymbol{t}}\left(\widehat{g}_{i} f_{i}\right) \leq \operatorname{deg}_{\boldsymbol{t}}\left(\bar{g}_{i} f_{i}\right)$. Therefore

$$
\begin{aligned}
\operatorname{deg}_{\boldsymbol{x}}\left(g_{i} f_{i}\right) \leq \operatorname{deg}_{\boldsymbol{x}}\left(\widehat{g}_{i} f_{i}\right)+\operatorname{deg}_{\boldsymbol{x}}(g) \max _{l} \operatorname{deg}_{y}\left(\widehat{g}_{l}\right) \leq 4\left(\prod_{j=0}^{\min \{s, r+1\}} d_{j}\right) \operatorname{deg}(V), \\
\operatorname{deg}_{\boldsymbol{t}}\left(g_{i} f_{i}\right) \leq \operatorname{deg}_{\boldsymbol{t}}\left(\widehat{g}_{i} f_{i}\right)+\operatorname{deg}_{\boldsymbol{t}}(g) \max _{l} \operatorname{deg}_{y}\left(\widehat{g}_{l}\right) \\
\leq 2\left(\prod_{j=0}^{\min \{s, r+1\}} d_{j}\right)\left(\mathrm{h}(V)+\operatorname{deg}(V)\left(\frac{3 h_{0}}{2 d_{0}}+\sum_{\ell=1}^{\min \{s, r+1\}} \frac{h}{d_{\ell}}\right)\right),
\end{aligned}
$$

as stated.

REMARK 4.27. - In the previous argument, the use of a differentiated version of the effective weak Nullstellensatz was crucial. Otherwise, the obtained bounds for the Noether exponent $\mu$ would have depended on the degree of $g$, as for instance in [5], and the bounds for the height of the $g_{i}$ 's would have been considerably worse.

\subsection{Nullstellensätze over $\mathbb{Z}$}

In this section we present different arithmetic Nullstellensätze over $\mathbb{Z}$ for polynomials depending on groups of parameters. As before, let $\boldsymbol{t}_{l}=\left\{t_{l, 1}, \ldots, t_{l, p_{l}}\right\}$ be a group of variables for $1 \leq l \leq m$ and set $\boldsymbol{t}=\left\{\boldsymbol{t}_{1}, \ldots, \boldsymbol{t}_{m}\right\}$. Write also $\boldsymbol{x}=\left\{x_{1}, \ldots, x_{n}\right\}$.

Theorem 4.28. - Let $V \subset \mathbb{A}_{\mathbb{Q}}^{n}$ be a $\mathbb{Q}$-variety of pure dimension $r$ and $f_{1}, \ldots, f_{s} \in \mathbb{Z}[\boldsymbol{t}, \boldsymbol{x}] \backslash \mathbb{Z}[\boldsymbol{t}]$ a family of $s \leq r+1$ polynomials without common zeros in $V_{\mathbb{Q}(\boldsymbol{t})}(\overline{\mathbb{Q}(\boldsymbol{t})})$. Set $d_{j}=\operatorname{deg}_{\boldsymbol{x}}\left(f_{j}\right), \delta_{l, j}=\operatorname{deg}_{\boldsymbol{t}_{l}}\left(f_{j}\right)$ and $h_{j}=\mathrm{h}\left(f_{j}\right)$ for $1 \leq j \leq s$ and $1 \leq l \leq m$. Then there exist $\alpha \in \mathbb{Z}[\boldsymbol{t}] \backslash\{0\}$ and $g_{1}, \ldots, g_{s} \in \mathbb{Z}[\boldsymbol{t}, \boldsymbol{x}]$ such that

$$
\alpha=g_{1} f_{1}+\cdots+g_{s} f_{s} \quad \text { on } V_{\mathbb{Q}(\boldsymbol{t})}
$$

with 


$$
\begin{aligned}
\text { - } \operatorname{deg}_{\boldsymbol{x}}\left(g_{i} f_{i}\right) \leq\left(\prod_{j=1}^{s} d_{j}\right) \operatorname{deg}(V), & \\
\text { - } \operatorname{deg}_{\boldsymbol{t}_{l}}(\alpha), \operatorname{deg}_{\boldsymbol{t}_{l}}\left(g_{i} f_{i}\right) \leq & \left(\prod_{j=1}^{s} d_{j}\right) \operatorname{deg}(V) \sum_{\ell=1}^{s} \frac{\delta_{l, \ell}}{d_{\ell}} \quad \text { for } 1 \leq l \leq m, \\
\text { - } \mathrm{h}(\alpha), \mathrm{h}\left(g_{i}\right)+\mathrm{h}\left(f_{i}\right) \leq & \left(\prod_{j=1}^{s} d_{j}\right)(\widehat{\mathrm{h}}(V)+\operatorname{deg}(V)((3 r+7) \log (n+3) \\
& \left.\left.+\sum_{\ell=1}^{s} \frac{1}{d_{\ell}}\left(h_{\ell}+\log \left(\# \operatorname{supp}\left(f_{\ell}\right)\right)+2 \sum_{l=1}^{m} \delta_{l, \ell} \log \left(p_{l}+1\right)\right)\right)\right) .
\end{aligned}
$$

Proof. - We apply again the construction explained in $\S 4$.1 to the $\operatorname{ring} A:=\mathbb{Z}\left[\boldsymbol{t}_{1}, \ldots, \boldsymbol{t}_{m}\right]$, the variety $V$ and the polynomials $f_{j}$. As a result, we obtain $\alpha \in A^{\times}$and polynomials $g_{i}$ satisfying the Bézout identity (4.29). The bounds for their $\boldsymbol{x}$-degree and $\boldsymbol{t}_{l}$-degree follow from Theorem 4.11, since $\operatorname{deg}\left(V_{\mathbb{Q}(\boldsymbol{t})}\right)=\operatorname{deg}(V)$ and $\mathrm{h}_{\boldsymbol{t}_{l}}\left(V_{\mathbb{Q}(\boldsymbol{t})}\right)=0$ by Proposition 2.13(2). So, we only have to bound the height of these polynomials.

We will use the notations introduced in $\$ 4$.1. Let $\boldsymbol{u}_{i}$ denote the group of coefficients of the general linear forms $\ell_{i}=u_{i, 1} x_{1}+\cdots+u_{i, n} x_{n}$ in (4.4) and set $\boldsymbol{u}=\left\{\boldsymbol{u}_{1}, \ldots, \boldsymbol{u}_{r+1}\right\}$. Consider the minimal polynomial $E \in \mathbb{Z}[\boldsymbol{t}, \boldsymbol{u}][\boldsymbol{y}, z]$ of $z$ with respect to the map $\pi \circ \varphi$, primitive with respect to $\mathbb{Z}[\boldsymbol{t}, \boldsymbol{u}]$. We expand it in two different ways as follows:

$$
E=\alpha_{\mathbf{0}, 0} z^{\delta}+\sum_{j=1}^{\delta} \sum_{\boldsymbol{a} \in \mathbb{N}^{r+1}} \alpha_{\boldsymbol{a}, j} \boldsymbol{y}^{\boldsymbol{a}} z^{\delta-j}=\sum_{\boldsymbol{a} \in \mathbb{N}^{r+1}} \alpha_{\boldsymbol{a}} \boldsymbol{y}^{\boldsymbol{a}}
$$

with $\alpha_{\boldsymbol{a}, j} \in \mathbb{Z}[\boldsymbol{t}, \boldsymbol{u}]$ such that $\alpha_{\mathbf{0}, 0} \neq 0$ and $\alpha_{\boldsymbol{a}}=\sum_{k=0}^{\delta} \alpha_{\boldsymbol{a}, k} z^{\delta-k} \in \mathbb{Z}[\boldsymbol{t}, \boldsymbol{u}, z]$. Set $\operatorname{supp}(E)=\left\{\boldsymbol{a}: \alpha_{\boldsymbol{a}} \neq 0\right\}$ and recall that, for $1 \leq i \leq s$,

$$
\widetilde{g}_{i}=-\sum_{j=1}^{\delta} \sum_{\boldsymbol{a} \in \operatorname{supp}(E)} \sum_{\boldsymbol{b}} \alpha_{\boldsymbol{a}, j}\left(\left(\prod_{k=1}^{r+1}\left(\begin{array}{l}
a_{k} \\
b_{k}
\end{array}\right) \ell_{k}^{a_{k}-b_{k}}\right) f_{1}^{b_{1}} \cdots f_{i-1}^{b_{i-1}} f_{i}^{b_{i}-1}\right) \in \mathbb{Z}[\boldsymbol{t}, \boldsymbol{u}][\boldsymbol{x}],
$$

the last sum being indexed by all $\boldsymbol{b} \in \mathbb{N}^{r+1}$ such that $|\boldsymbol{b}|=j, b_{k} \leq a_{k}$ for $1 \leq k \leq i, b_{k}=0$ for $i+1 \leq k \leq r+1$ and $b_{i} \geq 1$. The constant $\alpha$ and polynomials $g_{i}$ are obtained as the coefficient of some monomial in $\boldsymbol{u}$ in $\alpha_{\mathbf{0}, 0}$ and $\widetilde{g}_{i}$, respectively. Hence,

$$
\mathrm{h}(\alpha) \leq \mathrm{h}\left(\alpha_{\mathbf{0}, 0}\right), \quad \mathrm{h}\left(g_{i}\right)+\mathrm{h}\left(f_{i}\right) \leq \mathrm{h}\left(\widetilde{g}_{i}\right)+\mathrm{h}\left(f_{i}\right) .
$$

We have

$$
\sum_{j, \boldsymbol{a}, \boldsymbol{b}} \prod_{k=1}^{r+1}\left(\begin{array}{l}
a_{k} \\
b_{k}
\end{array}\right) \leq \sum_{\boldsymbol{a} \in \operatorname{supp}(E)} \sum_{\boldsymbol{b} \leq \boldsymbol{a}} \prod_{k=1}^{r+1}\left(\begin{array}{l}
a_{k} \\
b_{k}
\end{array}\right)=\sum_{\boldsymbol{a} \in \operatorname{supp}(E)} 2^{|\boldsymbol{a}|} \leq \# \operatorname{supp}(E) 2^{\operatorname{deg}_{\boldsymbol{y}}(E)} .
$$

Hence, by Lemma 2.37(1),

$$
\begin{aligned}
\mathrm{h}\left(\widetilde{g}_{i}\right)+\mathrm{h}\left(f_{i}\right) \leq \max _{\boldsymbol{a}, j}\left\{\mathrm{~h}\left(\alpha_{\boldsymbol{a}, j}\left(\prod_{k=1}^{r+1} \ell_{k}^{a_{k}-b_{k}}\right) f_{1}^{b_{1}} \cdots f_{i-1}^{b_{i-1}} f_{i}^{b_{i}-1}\right)\right\} \\
+\mathrm{h}\left(f_{i}\right)+\log \left(\# \operatorname{supp}(E) 2^{\operatorname{deg}_{\boldsymbol{y}}(E)}\right) .
\end{aligned}
$$


Set $\boldsymbol{d}=\left(d_{1}, \ldots, d_{s}, 1, \ldots, 1\right), \boldsymbol{\delta}_{l}=\left(\delta_{l, 1}, \ldots, \delta_{l, s}, 0, \ldots, 0\right)$ for $1 \leq l \leq m$ and $\boldsymbol{h}=\left(h_{1}, \ldots, h_{s}, 0, \ldots, 0\right)$. Using Lemma $2.37(2)$,

$$
\begin{aligned}
\mathrm{h}\left(\alpha_{\boldsymbol{a}, j}\left(\prod_{k=1}^{r+1} \ell_{k}^{a_{k}-b_{k}}\right)\right. & \left.f_{1}^{b_{1}} \cdots f_{i-1}^{b_{i-1}} f_{i}^{b_{i}-1}\right) \leq \mathrm{h}\left(\alpha_{\boldsymbol{a}, j}\right)+|\boldsymbol{a}| \log (n) \\
& +\left\langle\boldsymbol{h}-\boldsymbol{e}_{i}, \boldsymbol{a}\right\rangle+\langle\boldsymbol{d}, \boldsymbol{a}\rangle \log (n+1)+\sum_{l=1}^{m}\left\langle\boldsymbol{\delta}_{l}, \boldsymbol{a}\right\rangle \log \left(p_{l}+1\right),
\end{aligned}
$$

since $\log \left\|\ell_{k}\right\|_{1}=\log (n), \log \left\|f_{j}\right\|_{1} \leq \mathrm{h}\left(f_{j}\right)+d_{j} \log (n+1)+\sum_{l=1}^{m} \delta_{l, j} \log \left(p_{l}+1\right)$ and $\boldsymbol{b} \leq \boldsymbol{a}$.

By Lemma 4.8, $E \in \mathbb{Z}[\boldsymbol{t}, \boldsymbol{u}, z][\boldsymbol{y}]$ is a primitive and squarefree polynomial defining the closure of the image of the map $\psi$ in (4.10). Hence, we can apply Theorem 3.15 to bound its partial degrees and height. We have $\operatorname{deg}_{\boldsymbol{x}}\left(q_{j}\right)=d_{j}, \operatorname{deg}_{\boldsymbol{t}_{l}}\left(q_{j}\right)=\delta_{l, j}$ and $\mathrm{h}\left(q_{j}\right)=h_{j}$ for $1 \leq j \leq s$ while $\operatorname{deg}_{\boldsymbol{x}}\left(q_{j}\right)=1, \operatorname{deg}_{\boldsymbol{t}_{l}}\left(q_{j}\right)=\mathrm{h}\left(q_{j}\right)=0$ for $s+1 \leq j \leq r+1$. The partial degree of $q_{j}$ in the group of $(r+1) n+1$ variables $\boldsymbol{u} \cup\{z\}$ is equal to 1 . Set $D=\prod_{j=1}^{s} d_{j}$. Then, a direct application of Theorem 3.15 gives, for all $\boldsymbol{a} \in \operatorname{supp}(E)$,

$$
\langle\boldsymbol{d}, \boldsymbol{a}\rangle \leq D \operatorname{deg}(V), \quad \operatorname{deg}_{\boldsymbol{t}_{l}}\left(\alpha_{\boldsymbol{a}}\right)+\left\langle\boldsymbol{\delta}_{l}, \boldsymbol{a}\right\rangle \leq D \operatorname{deg}(V) \sum_{\ell=1}^{s} \frac{\delta_{l, \ell}}{d_{\ell}} \quad \text { for } 1 \leq l \leq m
$$

and

$$
\begin{aligned}
\mathrm{h}\left(\alpha_{\boldsymbol{a}}\right)+ & \langle\boldsymbol{h}, \boldsymbol{a}\rangle \leq D\left(\widehat{\mathrm{h}}(V)+\operatorname{deg}(V)\left(\log (r+2)+\sum_{\ell=1}^{s} \frac{1}{d_{\ell}}\left(h_{\ell}+\log \left(\# \operatorname{supp}\left(f_{\ell}\right)+n+2\right)\right.\right.\right. \\
& \left.\left.\left.+\sum_{l=1}^{m} \delta_{l, \ell} \log \left(p_{l}+1\right)\right)+(r+1-s) \log (n+2)+(r+1) \log ((r+1) n+2)\right)\right) .
\end{aligned}
$$

Using the inequalities $\log \left(\# \operatorname{supp}\left(f_{\ell}\right)+n+2\right) \leq \log \left(\# \operatorname{supp}\left(f_{\ell}\right)\right)+\log (n+3)$ and $\log ((r+1) n+2) \leq 2 \log (n+1)$ together with

$\log (r+2)+s \log (n+3)+(r+1-s) \log (n+2)+2(r+1) \log (n+1) \leq(3 r+4) \log (n+3)$, we deduce

$$
\begin{aligned}
\mathrm{h}\left(\alpha_{\boldsymbol{a}}\right)+\langle\boldsymbol{h}, \boldsymbol{a}\rangle \leq D(\widehat{\mathrm{h}}(V)+\operatorname{deg}(V)((3 r+4) \log (n+3) \\
\left.\left.\quad+\sum_{\ell=1}^{s} \frac{1}{d_{\ell}}\left(h_{\ell}+\log \left(\# \operatorname{supp}\left(f_{\ell}\right)\right)+\sum_{l=1}^{m} \delta_{l, \ell} \log \left(p_{l}+1\right)\right)\right)\right) .
\end{aligned}
$$

Moreover, we get from (4.33) that

$$
\log \left(\# \operatorname{supp}(E) 2^{\operatorname{deg}_{\boldsymbol{y}}(E)}\right)+|\boldsymbol{a}| \log (n)+\langle\boldsymbol{d}, \boldsymbol{a}\rangle \log (n+1) \leq 3 D \operatorname{deg}(V) \log (n+2) .
$$

The statement follows from (4.30), (4.31), (4.32), (4.34) and this inequality.

Theorem 1 in the introduction follows from the case $m=0$ in the previous result, noticing that for a polynomial $f \in \mathbb{Z}[\boldsymbol{x}]$ of degree $d$ it holds $\#(\operatorname{supp}(f)) \leq(n+1)^{d}$.

ExAMPLE 4.35. - Let $d_{1}, \ldots, d_{n}, H \geq 1$ and set

$$
\begin{aligned}
f_{1}=x_{1}^{d_{1}}, f_{2}= & x_{1} x_{n}^{d_{2}-1}-x_{2}^{d_{2}}, \ldots, \\
& f_{n-1}=x_{n-2} x_{n}^{d_{n-1}-1}-x_{n-1}^{d_{n-1}}, f_{n}=x_{n-1} x_{n}^{d_{n}-1}-H \in \mathbb{Z}\left[x_{1}, \ldots, x_{n}\right] .
\end{aligned}
$$


It is a system of polynomials without common zeros in $\overline{\mathbb{Q}}^{n}$. Theorem 4.28 implies that there is a Bézout identity $\alpha=g_{1} f_{1}+\cdots+g_{n} f_{n}$ with $\operatorname{deg}\left(g_{i} f_{i}\right) \leq d_{1} \cdots d_{n}$ and

$$
\log (\alpha), \mathrm{h}\left(g_{i}\right)+\mathrm{h}\left(f_{i}\right) \leq d_{1} \cdots d_{n-1} \log (H)+(4 n+7) \log (n+2) d_{1} \cdots d_{n} .
$$

On the other hand, let $u$ be an additional variable and consider the specialization of any such identity at $x_{i}=\gamma_{i}$ with

$$
\gamma_{1}=H^{d_{2} \cdots d_{n-1}} u^{d_{2} \cdots d_{n}-1}, \ldots, \gamma_{n-1}=H u^{d_{n}-1}, \gamma_{n}=1 / u
$$

We obtain $\alpha=g_{1}\left(\gamma_{1}, \ldots, \gamma_{n-1}, 1 / u\right) H^{d_{1} \cdots d_{n-1}} u^{d_{1} \cdots d_{n}-d_{1}}$. From this, we deduce the lower bounds

$$
\operatorname{deg}_{x_{n}}\left(g_{1} f_{1}\right) \geq d_{1} \cdots d_{n}, \quad \log (\alpha) \geq d_{1} \cdots d_{n-1} \log (H) .
$$

Hence, the height bound in (4.36) is optimal up to a term of size $O\left(n \log (n) d_{1} \cdots d_{n}\right)$.

We next analyze Example 4.18 from the point of view of heights.

EXAmple 4.37. - Let $V \subset \mathbb{A}_{\mathbb{Q}}^{n}$ be a $\mathbb{Q}$-variety of pure dimension $r$. For $1 \leq j \leq r+1$ and $d_{j} \geq 1$, consider again the general $n$-variate polynomial of degree $d_{j}$

$$
F_{j}=\sum_{|\boldsymbol{a}| \leq d_{j}} u_{j, \boldsymbol{a}} \boldsymbol{x}^{\boldsymbol{a}} \in \mathbb{Z}\left[\boldsymbol{u}_{j}\right]\left[x_{1}, \ldots, x_{n}\right]
$$

It follows from Example 4.18 that there exist $\lambda \in \mathbb{Z} \backslash\{0\}$ and $g_{i} \in \mathbb{Z}[\boldsymbol{u}]\left[x_{1}, \ldots, x_{n}\right]$ such that

$$
\lambda \operatorname{Res}_{d_{1}, \ldots, d_{r+1}}(\bar{V})=g_{1} F_{1}+\cdots+g_{r+1} F_{r+1} \quad \text { on } V
$$

satisfying the degree bounds in (4.19). For the height, we have that $\operatorname{deg}_{\boldsymbol{x}}\left(F_{j}\right)=d_{j}, \mathrm{~h}\left(F_{j}\right)=0$ and $\log \left(\# \operatorname{supp}\left(F_{j}\right)\right) \leq d_{j} \log (n+1)$. Furthermore, $\delta_{l, j}=\operatorname{deg}_{\boldsymbol{u}_{l}}\left(F_{j}\right)$ equals 1 if $l=j$ and 0 otherwise. Theorem 4.28 then implies

$$
\mathrm{h}\left(\operatorname{Res}_{d_{1}, \ldots, d_{r+1}}(\bar{V})\right), \mathrm{h}\left(g_{i}\right) \leq\left(\prod_{\ell=1}^{r+1} d_{\ell}\right)(\widehat{\mathrm{h}}(V)+(6 r+10) \log (n+3) \operatorname{deg}(V)) .
$$

The following result is a partial extension of Theorem 4.28 to an arbitrary number of polynomials. For simplicity, we state it for polynomials not depending on parameters.

Corollary 4.38. - Let $V \subset \mathbb{A}_{\mathbb{Q}}^{n}$ be a $\mathbb{Q}$-variety of pure dimension $r$ and $f_{1}, \ldots, f_{s} \in \mathbb{Z}[\boldsymbol{x}] \backslash \mathbb{Z}$ without common zeros in $V(\overline{\mathbb{Q}})$ such that $V\left(f_{s}\right)$ intersects $V$ properly. Set $d_{j}=\operatorname{deg}\left(f_{j}\right)$ for $1 \leq j \leq s$. Assume that $d_{1} \geq \cdots \geq d_{s-1}$ and that $d_{s}$ is arbitrary. Set also $h=\max _{1 \leq j \leq s-1} \mathrm{~h}\left(f_{j}\right)$ and $h_{s}=\mathrm{h}\left(f_{s}\right)$. Then there exist $\alpha \in \mathbb{Z} \backslash\{0\}$ and $g_{1}, \ldots, g_{s} \in \mathbb{Z}[\boldsymbol{x}]$ such that $\alpha=g_{1} f_{1}+\cdots+g_{s} f_{s}$ on $V$ with

- $\operatorname{deg}\left(g_{i} f_{i}\right) \leq\left(d_{s} \prod_{j=1}^{\min \{s-1, r\}} d_{j}\right) \operatorname{deg}(V)$,

- $\mathrm{h}(\alpha), \mathrm{h}\left(g_{i}\right)+\mathrm{h}\left(f_{i}\right) \leq\left(d_{s} \prod_{j=1}^{\min \{s-1, r\}} d_{j}\right)\left(\widehat{\mathrm{h}}(V)+\operatorname{deg}(V)\left(\frac{h_{s}}{d_{s}}+\sum_{\ell=1}^{\min \{s-1, r\}} \frac{h}{d_{\ell}}\right.\right.$

$$
+(6 r+9) \log (n+3)+3 r \log (\max \{1, s-r\}))) .
$$


Proof. - The proof follows closely the lines of that of Corollary 4.20. If $s \leq r+1$, the result follows from the case $m=0$ in Theorem 4.11. Hence, we only have to consider the case $s \geq r+2$. Set

$$
\bar{f}_{j}=f_{j}+v_{j, 1} f_{r+1}+\cdots+v_{j, s-r-1} f_{s-1} \quad \text { for } 1 \leq j \leq r, \quad \bar{f}_{r+1}=f_{s}
$$

for a group $\boldsymbol{v}=\left\{v_{j, i}\right\}_{j, i}$ of $p:=r(s-r-1)$ variables. It is a system of polynomials without common zeros in $V_{\mathbb{Q}(\boldsymbol{v})}(\overline{\mathbb{Q}(\boldsymbol{v})})$. We have that $\operatorname{deg}_{\boldsymbol{x}}\left(\bar{f}_{j}\right)=d_{j}, \operatorname{deg}_{\boldsymbol{v}}\left(\bar{f}_{j}\right)=1$ and $\mathrm{h}\left(\bar{f}_{j}\right) \leq h$ for $1 \leq j \leq r$ while $\operatorname{deg}_{\boldsymbol{x}}\left(\bar{f}_{r+1}\right)=d_{s}, \operatorname{deg}_{\boldsymbol{v}}\left(\bar{f}_{r+1}\right)=0$ and $\mathrm{h}\left(\bar{f}_{s}\right)=h_{s}$. By Theorem 4.28, there are $\bar{\alpha} \in \mathbb{Z}[\boldsymbol{v}] \backslash\{0\}$ and $\bar{g}_{i} \in \mathbb{Z}[\boldsymbol{v}, \boldsymbol{x}]$ such that $\bar{\alpha}=\bar{g}_{1} \bar{f}_{1}+\cdots+\bar{g}_{r+1} \bar{f}_{r+1}$ on $V_{\mathbb{Q}(\overline{\boldsymbol{v}})}$ with $\operatorname{deg}_{\boldsymbol{x}}\left(\bar{g}_{i} \bar{f}_{i}\right) \leq\left(d_{s} \prod_{j=1}^{r} d_{j}\right) \operatorname{deg}(V)$ and

$$
\begin{aligned}
\mathrm{h}(\bar{\alpha}), \mathrm{h}\left(\bar{g}_{i}\right)+\mathrm{h}\left(\bar{f}_{i}\right) \leq\left(d_{s} \prod_{j=1}^{r} d_{j}\right)\left(\widehat{\mathrm{h}}(V)+\operatorname{deg}(V)\left(\frac{1}{d_{s}}\left(h_{s}+\log \left(\# \operatorname{supp}\left(f_{s}\right)\right)\right)\right.\right. \\
\left.\left.+\sum_{\ell=1}^{r} \frac{1}{d_{\ell}}\left(h+\log \left(\# \operatorname{supp}\left(\bar{f}_{\ell}\right)\right)+2 \log (p+1)\right)+(3 r+7) \log (n+3)\right)\right) .
\end{aligned}
$$

We have that $\log (p+1) \leq \log (n+2)+\log (s-r)$ and $\# \operatorname{supp}\left(\bar{f}_{\ell}\right) \leq(s-r)(n+1)^{d_{\ell}}$ for $1 \leq \ell \leq r$ while $\# \operatorname{supp}\left(\bar{f}_{r+1}\right) \leq(n+1)^{d_{s}}$. Therefore,

$$
\begin{aligned}
\mathrm{h}(\bar{\alpha}), \mathrm{h}\left(\bar{g}_{i}\right)+\mathrm{h}\left(\bar{f}_{i}\right) \leq\left(d_{s} \prod_{j=1}^{r} d_{j}\right)\left(\widehat{\mathrm{h}}(V)+\operatorname{deg}(V)\left(\frac{h_{s}}{d_{s}}\right.\right. & +\sum_{\ell=1}^{r} \frac{h}{d_{\ell}}+3 r \log (s-r) \\
& +(6 r+8) \log (n+3))) .
\end{aligned}
$$

Set $\widetilde{g}_{i}=\bar{g}_{i}$ for $1 \leq i \leq r, \widetilde{g}_{i}=\sum_{k=1}^{r} v_{k, i-r} \bar{g}_{k}$ for $r+1 \leq i \leq s-1$ and $\widetilde{g}_{s}=\bar{g}_{r+1}$, then

$$
\widetilde{g}_{1} f_{1}+\cdots+\widetilde{g}_{s} f_{s}=\bar{g}_{1} \bar{f}_{1}+\cdots+\bar{g}_{r+1} \bar{f}_{r+1}=\bar{\alpha}
$$

with $\mathrm{h}\left(\widetilde{g}_{i}\right)+\mathrm{h}\left(f_{i}\right) \leq \max _{k}\left\{\mathrm{~h}\left(\bar{g}_{k}\right)+\mathrm{h}\left(\bar{f}_{k}\right)\right\}+\log (r)$ for all $i$. By taking the coefficients of a suitable monomial in $\boldsymbol{v}$, we extract from (4.40) a Bézout identity $\alpha=g_{1} f_{1}+\cdots+g_{s} f_{s}$ on $V$. By (4.39), these polynomials satisfy the stated degree and height bounds.

We finally prove the arithmetic strong Nullstellensatz presented in the introduction.

Proof of Theorem 2. - We use the same notations of the proof of Theorem 4.22 for $k=\mathbb{Q}$ and $p=0$. The proof of this corollary already gives the stated bounds for the degree of the $g_{i}$ 's and the exponent $\mu$. Hence, it only remains to bound the height in the identity $\alpha g^{\mu}=g_{1} f_{1}+\cdots+g_{s} f_{s}$ on $V$.

Corollary 4.38 applied to $W=V \times \mathbb{A}^{1}$ and $1-y^{d_{0}} g, f_{1}, \ldots, f_{s}$ and Lemma 3.16 imply

$$
\begin{aligned}
\mathrm{h}(\alpha), \mathrm{h}\left(\bar{g}_{i}\right)+\mathrm{h}\left(f_{i}\right) \leq 2 & d_{0} D\left(\widehat{\mathrm{h}}(V)+\operatorname{deg}(V)\left(\frac{h_{0}}{2 d_{0}}+\sum_{\ell=1}^{\min \{s, r+1\}} \frac{h}{d_{\ell}}\right.\right. \\
& +(6 r+15) \log (n+4)+3(r+1) \log (\max \{1, s-r\}))),
\end{aligned}
$$


with $D:=\prod_{j=1}^{\min \{s, r+1\}} d_{j}$. From this, we deduce the bound for the height of $\alpha$. Let $\widehat{g}_{i}$ be as in (4.25). If we write $\widehat{g}_{i}=\sum_{\boldsymbol{a}, j} \alpha_{\boldsymbol{a}, j} \boldsymbol{x}^{\boldsymbol{a}} y^{j}$, then $g_{i}=\sum_{\boldsymbol{a}, j} \alpha_{\boldsymbol{a}, j} \boldsymbol{x}^{\boldsymbol{a}} g^{\max _{l}\left\{\operatorname{deg}_{y}\left(\widehat{g}_{l}\right)\right\}-j}$. Using Lemma 2.37 we deduce that

$$
\begin{aligned}
\mathrm{h}\left(g_{i}\right) & \leq \max _{\boldsymbol{a}, j}\left\{\mathrm{~h}\left(\alpha_{\boldsymbol{a}, j} \boldsymbol{x}^{\boldsymbol{a}} g^{\max _{l}\left\{\operatorname{deg}_{y}\left(\widehat{g}_{l}\right)\right\}-j}\right)\right\}+\log \left(\# \operatorname{supp}\left(\widehat{g}_{i}\right)\right) \\
& \leq \mathrm{h}\left(\widehat{g}_{i}\right)+\max _{l}\left\{\operatorname{deg}_{y}\left(\widehat{g}_{l}\right)\right\}\left(h_{0}+\log (n+1) d_{0}\right)+\log \left(\# \operatorname{supp}\left(\widehat{g}_{i}\right)\right) .
\end{aligned}
$$

Observe that $\mathrm{h}\left(\widehat{g}_{i}\right) \leq \mathrm{h}\left(\bar{g}_{i}\right) \quad$ and $\quad \# \operatorname{supp}\left(\widehat{g}_{i}\right) \leq(n+2)^{\operatorname{deg}_{\boldsymbol{x}, y}\left(\widehat{g}_{i}\right)}$. Moreover, $\operatorname{deg}_{y}\left(\widehat{g}_{i}\right) \leq 2 D \operatorname{deg}(V)$ and $\operatorname{deg}_{\boldsymbol{x}, y}\left(\widehat{g}_{i}\right) \leq 2 d_{0} D \operatorname{deg}(V)$, as shown in the proof of Theorem 4.22. Therefore, $\mathrm{h}\left(g_{i}\right)+\mathrm{h}\left(f_{i}\right)$ is bounded above by

$$
\left.\mathrm{h}\left(\bar{g}_{i}\right)+\mathrm{h}\left(f_{i}\right)+2 D \operatorname{deg}(V)\left(h_{0}+\log (n+1) d_{0}\right)+2 d_{0} D \operatorname{deg}(V) \log (n+2)\right) .
$$

The stated bound for $\mathrm{h}\left(g_{i}\right)+\mathrm{h}\left(f_{i}\right)$ follows from this and (4.41).

\section{REFERENCES}

[1] M. Aschenbrenner, Ideal membership in polynomial rings over the integers, J. Amer. Math. Soc. 17 (2004), 407-441.

[2] C. A. Berenstein, A. Yger, Effective Bézout identities in $\mathbb{Q}\left[z_{1}, \ldots, z_{n}\right]$, Acta Math. 166 (1991), 69-120.

[3] Y. F. Bilu, M. Strambi, Quantitative Riemann existence theorem over a number field, Acta Arith. 145 (2010), 319-339.

[4] E. Bombieri, J. Bourgain, S. V. Konyagin, Roots of polynomials in subgroups of $\mathbb{F}_{p}^{*}$ and applications to congruences, Int. Math. Res. Not. 2009 (2009), 802-834.

[5] W. D. Brownawell, Bounds for the degrees in the Nullstellensatz, Ann. of Math. 126 (1987), 577-591.

[6] W. D. Brownawell, The Hilbert Nullstellensatz, inequalities for polynomials, and algebraic independence, in Introduction to algebraic independence theory, Lecture Notes in Math. 1752, Springer, 2001, 239-248.

[7] J. I. Burgos Gil, P. Philippon, M. Sombra, Arithmetic geometry of toric varieties. Metrics, measures and heights, preprint arXiv:1105.5584.

[8] W.-L. Chow, B. L. van der W aerden, Zur algebraischen Geometrie. IX, Math. Ann. 113 (1937), 692-704.

[9] X. Dahan, A. Kadri, É. Schost, Bit-size estimates for triangular sets in positive dimension, J. Complexity 28 (2012), 109-135.

[10] S. David, P. Philippon, Minorations des hauteurs normalisées des sous-variétés des tores, Ann. Scuola Norm. Sup. Pisa Cl. Sci. 28 (1999), 489-543.

[11] W. Fulton, Intersection theory, Ergebn. Math. Grenzg. 2, Springer, 1984.

[12] I. M. Gel'Fand, M. M. Kapranov, A. V. Zelevinsky, Discriminants, resultants, and multidimensional determinants, Mathematics: Theory \& Applications, Birkhäuser, 1994.

[13] R. Hartshorne, Algebraic geometry, Graduate Texts in Math. 52, Springer, 1977.

[14] Z. JeloneK, On the effective Nullstellensatz, Invent. Math. 162 (2005), 1-17. 
[15] J.-P. Jounnolou, Théorèmes de Bertini et applications, Progress in Math. 42, Birkhäuser, 1983.

[16] P. KoIRan, Hilbert's Nullstellensatz is in the polynomial hierarchy, J. Complexity 12 (1996), 273-286.

[17] A. Kresch, Y. Tschinkel, Effectivity of Brauer-Manin obstructions, Adv. Math. 218 (2008), 1-27.

[18] T. KRICK, L. M. PARdo, A computational method for Diophantine approximation, in Algorithms in algebraic geometry and applications (Santander, 1994), Progr. Math. 143, Birkhäuser, 1996, 193-253.

[19] T. Krick, L. M. Pardo, M. Sombra, Sharp estimates for the arithmetic Nullstellensatz, Duke Math. J. 109 (2001), 521-598.

[20] S. Lang, Fundamentals of Diophantine geometry, Springer, 1983.

[21] S. LAng, Algebra, third ed., Addison-Wesley Publishing Co., Inc., Reading, Mass., 1993.

[22] P. Lelong, Mesure de Mahler et calcul de constantes universelles pour les polynômes de $n$ variables, Math. Ann. 299 (1994), 673-695.

[23] F. S. Macaulay, Some formulae in elimination, Proc. London Math. Soc. 1 (1902), 3 27.

[24] V. Maillot, Géométrie d'Arakelov des variétés toriques et fibrés en droites intégrables, Mém. Soc. Math. France 80, 2000.

[25] P. Pedersen, B. Sturmfels, Product formulas for resultants and Chow forms, Math. Z. 214 (1993), 377-396.

[26] O. Perron, Algebra. I. Die Grundlagen, Walter de Gruyter \& Co., 1951.

[27] P. Philip pon, Critères pour l'indépendance algébrique, Publ. Math. I.H.É.S. 64 (1986), $5-52$.

[28] P. Philippon, Dénominateurs dans le théorème des zéros de Hilbert, Acta Arith. 58 (1991), 1-25.

[29] P. Philippon, Sur des hauteurs alternatives. I, Math. Ann. 289 (1991), 255-283.

[30] P. Philippon, Sur des hauteurs alternatives. III, J. Math. Pures Appl. 74 (1995), 345365.

[31] P. Philippon, M. Sombra, Hauteur normalisée des variétés toriques projectives, J. Inst. Math. Jussieu 7 (2008), 327-373.

[32] G. RÉMOND, Élimination multihomogène, in Introduction to algebraic independence theory, Lecture Notes in Math. 1752, Springer, 2001, 53-81.

[33] G. Rémond, Géométrie diophantienne multiprojective, in Introduction to algebraic independence theory, Lecture Notes in Math. 1752, Springer, 2001, 95-131.

[34] G. Rémond, Nombre de points rationnels des courbes, Proc. Lond. Math. Soc. 101 (2010), 759-794.

[35] F. Smietanski, A parametrized Nullstellensatz, in Computational algebraic geometry (Nice, 1992), Progr. Math. 109, Birkhäuser, 1993, 287-300.

[36] C. J. Sмүтн, A Kronecker-type theorem for complex polynomials in several variables, Canad. Math. Bull. 24 (1981), 447-452.

$4^{\mathrm{e}}$ SÉRIE - TOME $46-2013$ - No 4 
[37] M. Sombra, The height of the mixed sparse resultant, Amer. J. Math. 126 (2004), $1253-$ 1260.

[38] B. Teissier, Résultats récents d'algèbre commutative effective, Séminaire Bourbaki, vol. 1989/90, exposé no 718, Astérisque 189-190 (1990), 107-131.

(Manuscrit reçu le 28 novembre 2011; accepté, après révision, le 18 octobre 2012.)

\author{
Carlos D'Andrea \\ Departament d'Àlgebra i Geometria \\ Universitat de Barcelona \\ Gran Via 585 \\ 08007 Barcelona, Spain \\ E-mail: cdandrea@ub.edu \\ Teresa KRICK \\ Departamento de Matemática \\ Facultad de Ciencias Exactas y Naturales \\ Universidad de Buenos Aires \\ and \\ IMAS, CONICET \\ Ciudad Universitaria \\ 1428 Buenos Aires, Argentina \\ E-mail: krick@dm.uba.ar \\ Martín Sombra \\ ICREA and \\ Departament d'Àlgebra i Geometria \\ Universitat de Barcelona \\ Gran Via 585 \\ 08007 Barcelona, Spain \\ E-mail: sombra@ub.edu
}


